\title{
Direct Catalytic Asymmetric Synthesis of Cyclic Aminals from Aldehydes
}

\author{
Xu Cheng, Sreekumar Vellalath, Richard Goddard, and Benjamin List* \\ Max-Planck-Institut für Kohlenforschung,Kaiser-Wilhelm-Platz 1,D-45470, \\ Mülheim an der Ruhr, Germany, list@mpi-muelheim.mpg.de
}

\section{Supporting Information}

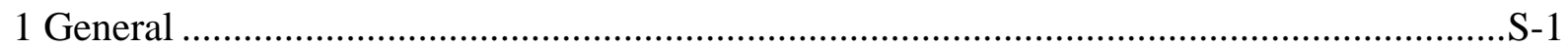

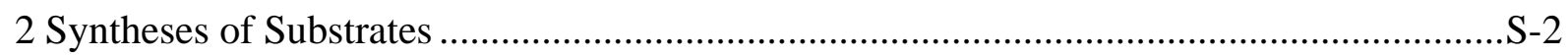

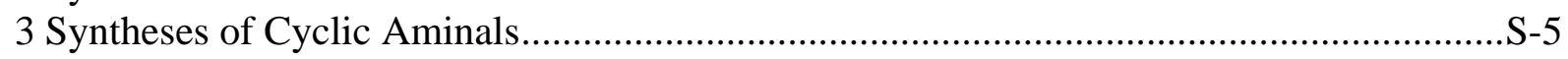

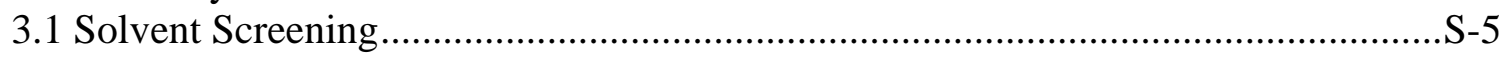

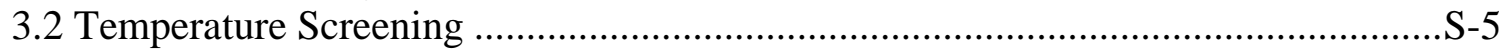

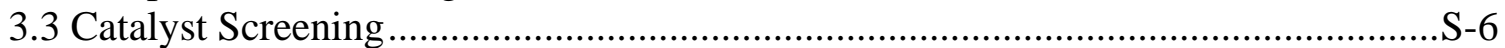

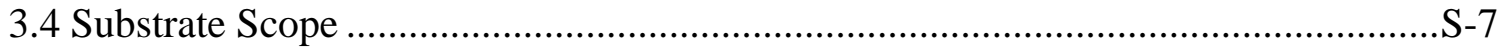

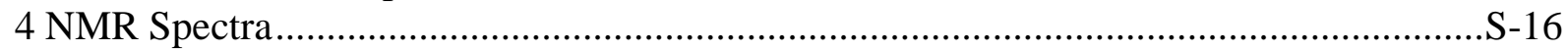

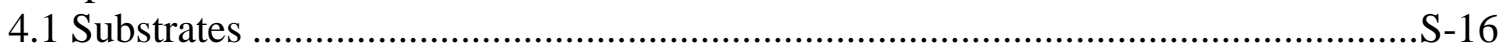

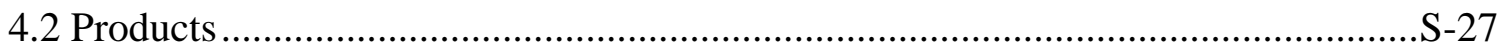

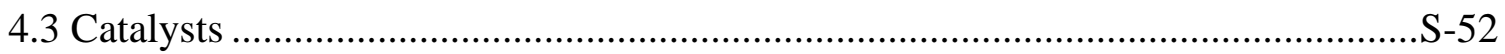

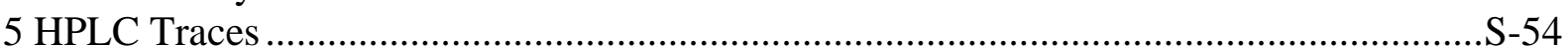

6 Preparation and Analysis of Single Crystal of Compound 3k ........................................

\section{General}

All reactions were carried out under argon atmosphere in oven dried glassware with magnetic stirring. Unless otherwise stated, all reagents were purchased from commercial suppliers and used without further purification. All solvents used in the reactions were distilled from appropriate drying agents prior to use. Analytical thin-layer chromatography (TLC) was performed on silica gel precoated glass plates (0.25 mm thickness, 60F-254, MN). Visualization was accomplished by irradiation with a UV light at $254 \mathrm{~nm}$. Chromatography was performed using silica gel $60(0.040-0.063 \mathrm{~mm})$ from Merck. Proton, carbon and phosphorous NMR spectra were recorded on Bruker AV-500, Bruker AV-400 or DPX-300 spectrometer in deutrated solvent. Proton chemical shifts are reported in ppm $(\delta)$ relative to tetramethylsilane (TMS) with the solvent resonance employed as the internal standard $\left(\mathrm{CDCl}_{3}\right.$, $\delta 7.26 \mathrm{ppm}$ ). Data are reported as follows: chemical shift, multiplicity (s = singlet, $\mathrm{d}=$ doublet, $\mathrm{q}=$ quartet, $\mathrm{m}=$ multiplet), coupling constants $(\mathrm{Hz})$ and integration. ${ }^{13} \mathrm{C}$ chemical shifts are reported in ppm from tetramethylsilane (TMS) with the solvent resonance as the internal 
standard $\left(\mathrm{CDCl}_{3}, \delta 77.0 \mathrm{ppm}\right) .{ }^{31} \mathrm{P}$ chemical shifts are reported in ppm from $\mathrm{H}_{3} \mathrm{PO}_{4}$ as external standard. Spectra were drawn with TOPSPIN automatically and analyzed with Mestrec. Mass spectra were obtained on a Finnigan MAT $8200(70 \mathrm{eV})$, accurate mass determinations were done on a Bruker APEX III FT-MS (7 $\mathrm{T}$ magnet). The enantiomeric excesses were determined by HPLC analysis employing a chiral stationary phase column specified in the individual experiment, by comparing the samples with the appropriate racemic mixtures.

\section{Syntheses of Substrates}

\section{Procedure 1}

2-Amino-4-methylbenzamide $\mathbf{1 h}$<smiles>Cc1ccc(C#N)c(N)c1</smiles>

1h

A mixture of 2-amino-4-methylbenzonitrile (2.26 g, $17.1 \mathrm{mmol})$ and $\mathrm{KOH}(4.8 \mathrm{~g}, 85 \%, 73$ $\mathrm{mmol})$ in $\mathrm{EtOH}(50 \mathrm{~mL})$ was refluxed for $24 \mathrm{~h}$. After evaporation of the solvent, the reaction mixture was dilute with water $(50 \mathrm{~mL})$ and extracted with ethyl acetate (20 mL X 3). The combined organic layer were dried $\left(\mathrm{MgSO}_{4}\right)$ and concentrated, followed by silca gel chromatography with ethyl acetate as eluent to give 2-amino-4-methylbenzamide $\mathbf{1 h}$ (2.2 g, 86\%). ${ }^{1} \mathrm{H}$ NMR (500 MHz, $\mathrm{CDCl}_{3}$ ) $\delta 2.26$ (s, 3H, $\mathrm{CH}_{3}$ ), 5.68 (s, 4H, $\mathrm{NH}_{2}$ ), 6.46 (d, $J=8.1 \mathrm{~Hz}$, $1 \mathrm{H}, \mathrm{ArH}), 6.50$ (s, 1H, ArH), 7.25 (d, $J=8.1 \mathrm{~Hz}, 1 \mathrm{H}, \mathrm{ArH}) .{ }^{13} \mathrm{C}$ NMR $\left(125 \mathrm{MHz}, \mathrm{CDCl}_{3}\right) \delta$ 21.4, 111.2, 117.6, 127.9, 143.7, 149.6, 171.4. MS (EI) m/z (\%) 150 (80), 133 (100), 106 (31), 104 (13), 78 (12), 77 (16). HRMS calculated for $\left(\mathrm{C}_{8} \mathrm{H}_{10} \mathrm{~N}_{2} \mathrm{O}\right)$ 150.079313, found 150.079473.<smiles>Cc1cccc(N)c1C(N)=O</smiles>

2-Amino-6-methylbenzamide $\mathbf{1} \mathbf{j}$ was also prepared according to procedure 1 from 2-amino-6methylbenzonitrile $(2.26 \mathrm{~g}, 17.1 \mathrm{mmol})$ as white solid in $81 \%$ yield. ${ }^{1} \mathrm{H}$ NMR $(500 \mathrm{MHz}$, $\left.\mathrm{CDCl}_{3}\right) \delta 2.39\left(\mathrm{~s}, 3 \mathrm{H}, \mathrm{CH}_{3}\right), 4.26\left(\mathrm{~s}, 2 \mathrm{H}, \mathrm{NH}_{2}\right), 5.75(\mathrm{~s}, 1 \mathrm{H}, \mathrm{NH}), 5.88(\mathrm{~s}, 1 \mathrm{H}, \mathrm{NH}), 6.55(\mathrm{~d}, J$ $=8.0 \mathrm{~Hz}, 1 \mathrm{H}, \mathrm{ArH}), 6.59$ (d, $J=7.5,1 \mathrm{H}, \mathrm{ArH}), 7.25(\mathrm{t}, J=8.0 \mathrm{~Hz}, 1 \mathrm{H}, \mathrm{ArH}) .{ }^{13} \mathrm{C}$ NMR $(75$ $\left.\mathrm{MHz}, \mathrm{CDCl}_{3}\right) \delta 20.4,113.9,120.6,121.4,130.3,135.4,144.9,171.4$. MS (EI) m/z (\%) 150 (43), 133 (100), 106 (24), 105 (12), 104 (14), 78 (10), 77 (13). HRMS calculated for $\left(\mathrm{C}_{8} \mathrm{H}_{10} \mathrm{~N}_{2} \mathrm{O}\right)$ 150.079314, found 150.079162.<smiles>NC(=O)c1c(N)cccc1Cl</smiles>

2-Amino-6-chlorobenzamide $\mathbf{1 l}$ was also prepared according to procedure 1 from 2-amino-6chlorobenzonitrile $(2.61 \mathrm{~g}, 17.1 \mathrm{mmol})$ as white solid in $83 \%$ yield. ${ }^{1} \mathrm{H}$ NMR $(500 \mathrm{MHz}$, $\left.\mathrm{CDCl}_{3}\right) \delta 4.36\left(\mathrm{~b}, 2 \mathrm{H}, \mathrm{NH}_{2}\right), 6.12(\mathrm{~s}, 1 \mathrm{H}, \mathrm{NH}), 6.21(\mathrm{~s}, 1 \mathrm{H}, \mathrm{NH}), 6.60(\mathrm{~d}, J=9.0 \mathrm{~Hz}, 1 \mathrm{H}$, ArH), 6.73 (d, $J=8.0 \mathrm{~Hz}, 1 \mathrm{H}, \operatorname{ArH}), 7.06$ (t, $J=8.0 \mathrm{~Hz}, 1 \mathrm{H}, \operatorname{ArH}) .{ }^{13} \mathrm{C}$ NMR $(125 \mathrm{MHz}$, 
$\left.\mathrm{CDCl}_{3}\right) \delta$ 115.1, 118.0, 118.9, 131.3, 148.2, 168.7. MS (EI) m/z (\%) 172 (20), 170 (64), 155 (32), 153 (100), 126 (38), 90 (22), 63 (13). HRMS calculated for $\left(\mathrm{C}_{7} \mathrm{H}_{7} \mathrm{~N}_{2} \mathrm{OCl}\right)$ 170.024688, found 170.024520 .

\section{Procedure 2}

2-Amino-5-methoxybenzamide 10<smiles>COc1ccc(N)c(C(=O)O)c1</smiles><smiles>COc1ccc(N)c(C(N)=O)c1</smiles>

A solution of 2-amino-5-methoxybenzoic acid (170 mg, $1 \mathrm{mmol}$ ) and 1,1'-carbonyldiimidazol (180 mg, 90\%, $1 \mathrm{mmol}$ ) in anhydrous THF (5 mL) was stirred at room temperature under argon for 2 hours, followed by the addition of ammonia (5 mL, 25\%, $73 \mathrm{mmol}$ ). After vigorous stirring for 6 hours, the reaction mixture was concentrated to remove THF. The residue was extracted with ethyl acetate three times. The combined organic solvent was dried $\left(\mathrm{MgSO}_{4}\right)$ and concentrated. The residue was purified by silica gel chromatography with ethyl acetate as eluent to give the 2-amino-5-methoxybenzamide 10 (104 mg, 60\%) as white solid. ${ }^{1} \mathrm{H}$ NMR (500 MHz, $\left.\mathrm{CDCl}_{3}\right) \delta 3.76\left(\mathrm{~s}, 3 \mathrm{H}, \mathrm{OCH}_{3}\right), 5.20\left(\mathrm{~s}, 2 \mathrm{H}, \mathrm{NH}_{2}\right), 5.84\left(\mathrm{~b}, 2 \mathrm{H}, \mathrm{NH}_{2}\right.$ ), 6.67 (d, $J=8.6 \mathrm{~Hz}, 1 \mathrm{H}, \mathrm{ArH}), 6.90-6.93(\mathrm{~m}, 2 \mathrm{H}, \mathrm{ArH}) .{ }^{13} \mathrm{C}$ NMR $\left(125 \mathrm{MHz}, \mathrm{CDCl}_{3}\right) \delta 56.1$, 112.4, 115.2, 119.0, 120.3, 143.2, 151.0, 171.2. MS (EI) m/z (\%) 166 (100), 149 (69), 134 (33), 121 (21), 106 (47), 52 (10). HRMS calculated for $\left(\mathrm{C}_{8} \mathrm{H}_{10} \mathrm{~N}_{2} \mathrm{O}_{2}\right)$ 166.074227, found 166.074097.<smiles>Cc1ccc(N)c(C(N)=O)c1</smiles>

2-Amino-5-methylbenzamide $\mathbf{1 i}$ was also prepared according to procedure 2 from 2-amino-5methylbenzoic acid (151 mg, $1 \mathrm{mmol}$ ) as white solid in 70\% yield. Mp 175-177 ${ }^{\circ} \mathrm{C}$. ${ }^{1} \mathrm{H}$ NMR (500 MHz, CD $\left.\mathrm{CD}_{3} \mathrm{OD}\right) \delta 2.23$ (s, 3H, $\mathrm{CH}_{3}$ ), 4.88 (b, 4H, NH ), 6.67 (d, $J=8.6 \mathrm{~Hz}, 1 \mathrm{H}, \mathrm{ArH}$ ), 7.04-7.06 (m, 1H, ArH), 7.35 (s, 1H, ArH). ${ }^{13} \mathrm{C}$ NMR (125 MHz, CD $\left.{ }_{3} \mathrm{OD}\right) \delta 20.3,116.2$, 118.6, 126.6, 129.6, 134.5, 148.5, 174.6. MS (EI) m/z (\%) 150 (83), 133 (100), 104 (34), 77 (17). HRMS calculated for $\left(\mathrm{C}_{8} \mathrm{H}_{10} \mathrm{~N}_{2} \mathrm{O}\right) 150.079310$, found 150.079295.<smiles>NC(=O)c1cc(Br)ccc1N</smiles>

2-Amino-5-bromobenzamide $\mathbf{1 m}$ was also prepared according to procedure 2 from 2-amino5-bromobenzoic acid (214 mg, $1 \mathrm{mmol}$ ) as white solid in $62 \%$ yield. Mp $187-189{ }^{\circ} \mathrm{C} .{ }^{1} \mathrm{H}$ NMR (500 MHz, CD $\left.{ }_{3} \mathrm{OD}\right) \delta 4.88$ (b, 4H, NH 2$), 6.68$ (d, $\left.J=8.6 \mathrm{~Hz}, 1 \mathrm{H}, \mathrm{ArH}\right), 7.26-7.28$ (m, $1 \mathrm{H}, \mathrm{ArH}), 7.67$ (s, $1 \mathrm{H}, \mathrm{ArH}) .{ }^{13} \mathrm{C}$ NMR $\left(125 \mathrm{MHz}, \mathrm{CD}_{3} \mathrm{OD}\right) \delta 107.5,117.0,119.9,132.0$, 136.1, 150.3, 173.2. MS (EI) m/z (\%) 216 (78), 214 (79), 199 (97), 197 (100), 172 (19), 171 (23), 170 (20), 169 (22), 90 (31), 63 (31). HRMS calculated for $\left(\mathrm{C}_{7} \mathrm{H}_{7} \mathrm{BrN}_{2} \mathrm{O}\right)$ 213.974187, found 213.973965.<smiles>NC(=O)c1cc(OC(F)(F)F)ccc1N</smiles> 
2-Amino-5-(trifluoromethoxy)benzamide 1p was also prepared according to procedure 2 from 2-amino-5-(trifluoromethoxy)benzoic acid (1.10 g, $5 \mathrm{mmol}$ ) as white solid in $70 \%$ yield. Mp 103-105 ${ }^{\circ} \mathrm{C} .{ }^{1} \mathrm{H}$ NMR (300 MHz, $\left.\mathrm{CDCl}_{3}\right) \delta 5.72$ (b, 4H, NH ), 6.67 (d, $\left.J=9.0 \mathrm{~Hz}, 1 \mathrm{H}, \mathrm{ArH}\right)$, $7.12(\mathrm{~d}, J=9.0 \mathrm{~Hz}, 1 \mathrm{H}, \mathrm{ArH}), 7.22$ (s, $1 \mathrm{H}, \mathrm{ArH}) .{ }^{13} \mathrm{C}$ NMR $\left(75 \mathrm{MHz}, \mathrm{CDCl}_{3}\right) \delta$ 12.6, 21.0, 111.6, 118.6, 121.8, 125.0, 141.2, 147.9, 172.2. MS (EI) m/z (\%) 220 (100), 203 (92), 176 (18), 134 (11), 106 (34). HRMS calculated for $\left(\mathrm{C}_{8} \mathrm{H}_{7} \mathrm{~N}_{2} \mathrm{O}_{2} \mathrm{~F}_{3}\right)$ 220.045962, found 220.045756.<smiles>Cc1ccc(C(N)=O)c(N)c1C</smiles>

2-Amino-3,4-dimethylbenzamide 1q was also prepared according to procedure 2 from 2amino-3,4-dimethylbenzoic acid (0.73 g, $5 \mathrm{mmol}$ ) as yellow solid in 81\% yield. Mp 166-168 ${ }^{\circ} \mathrm{C} .{ }^{1} \mathrm{H}$ NMR (500 MHz, $\left.\mathrm{CDCl}_{3}\right) \delta 2.06\left(\mathrm{~s}, 3 \mathrm{H}, \mathrm{CH}_{3}\right.$ ), 2.84 (s, 3H, $\mathrm{CH}_{3}$ ), $5.83\left(\mathrm{~b}, 4 \mathrm{H}, \mathrm{NH}_{2}\right.$ ), $6.51(\mathrm{~d}, J=8.1 \mathrm{~Hz}, 1 \mathrm{H}, \mathrm{ArH}), 7.17(\mathrm{~d}, J=8.1 \mathrm{~Hz}, 1 \mathrm{H}, \mathrm{ArH}) .{ }^{13} \mathrm{C}$ NMR $\left(125 \mathrm{MHz}, \mathrm{CDCl}_{3}\right) \delta$ 12.6, 21.0, 111.5, 118.2, 121.8, 125.0, 141.2, 147.9, 172.2. MS (EI) m/z (\%) 164 (87), 147 (53), 119 (100), 104 (23), 91 (20). HRMS calculated for $\left(\mathrm{C}_{9} \mathrm{H}_{12} \mathrm{~N}_{2} \mathrm{O}\right)$ 164.094961, found 164.094842.<smiles>Cc1cc(Cl)cc(C(N)=O)c1N</smiles>

2-Amino-3-methyl-5-chlorobenzamide 1r was also prepared according to procedure 2 from 2amino-3methyl-5-chlorobenzoic acid (185 mg, $1 \mathrm{mmol}$ ) as yellow solid in 75\% yield. Mp 168-170 ${ }^{\circ} \mathrm{C} .{ }^{1} \mathrm{H}$ NMR $\left(500 \mathrm{MHz}, \mathrm{CD}_{3} \mathrm{OD}\right) \delta 2.16$ (s, 3H, $\mathrm{CH}_{3}$ ), 4.88 (b, 4H, $\mathrm{NH}_{2}$ ), 7.44 (s, $1 \mathrm{H}$, $\mathrm{ArH}), 7.13$ (s, 1H, ArH). ${ }^{13} \mathrm{C}$ NMR (125 MHz, CD $\left.{ }_{3} \mathrm{OD}\right) \delta 17.5,120.8,126.9,127.2,133.8$, 147.9, 173.7. MS (EI) m/z (\%) 184 (100), 167 (67), 139 (81), 104 (39), 77 (24). HRMS calculated for $\left(\mathrm{C}_{8} \mathrm{H}_{9} \mathrm{ClN}_{2} \mathrm{O}\right)$ 184.040338, found 184.040147.

Procedure 3<smiles>N#Cc1c(N)cccc1F</smiles>

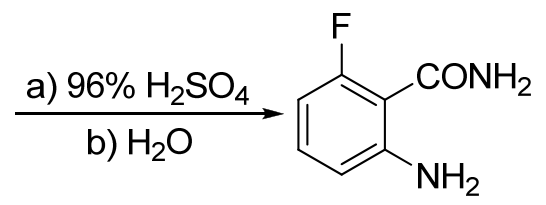

1n

2-Amino-6-fluorobenzamide 1n

To a solution of concentrated $\mathrm{H}_{2} \mathrm{SO}_{4}(10 \mathrm{~mL})$ was added 2-amino-6-fluorobenzonitrile (1.36 g, $10 \mathrm{mmol}$ ) at $65{ }^{\circ} \mathrm{C}$ and stirred for 3 hours at $65{ }^{\circ} \mathrm{C}$. Then the gray mixture was poured into ice and brought to $\mathrm{pH}=9$ by $20 \% \mathrm{NaOH}$ aqueous solution, followed by the extraction with ethyl acetate three times. The combined organic layer were dried $\left(\mathrm{MgSO}_{4}\right)$ and concentrated to give brown residue that was purified by silica gel chromatography with $\operatorname{Hex} / \mathrm{EA}(1: 1, \mathrm{v} / \mathrm{v})$ as eluent to give 2-amino-6-fluorobenzamide $(1.3 \mathrm{~g}, 84 \%)$ as white solid. Mp $120-122{ }^{\circ} \mathrm{C} .{ }^{1} \mathrm{H}$ NMR (300 MHz, $\mathrm{CDCl}_{3}$ ) $\delta 5.83$ (b, $1 \mathrm{H}, \mathrm{NH}$ ), 6.12 (s, 2H, $\mathrm{NH}_{2}$ ), 6.34 (dd, $J=5.0$ and $8.0 \mathrm{~Hz}$, 1H, ArH), 6.45 (d, $J=8.3 \mathrm{~Hz}, 1 \mathrm{H}, \mathrm{ArH}), 6.66$ (b, $1 \mathrm{H}, \mathrm{NH}), 7.11$ (dd, $J=8.2$ and $6.5 \mathrm{~Hz}, 1 \mathrm{H}$, $\mathrm{ArH}) .{ }^{13} \mathrm{C}$ NMR $\left(75 \mathrm{MHz}, \mathrm{CDCl}_{3}\right) \delta 102.3,102.5,102.6,102.9,113.0,113.1,132.7,132.9$, 152.1, 152.2, 161.0, 164.3, 168.3. MS (EI) m/z (\%) 154 (100), 137 (89), 110 (51), 83 (12). HRMS calculated for $\left(\mathrm{C}_{7} \mathrm{H}_{7} \mathrm{~N}_{2} \mathrm{OF}\right)$ 154.054241, found 154.054203. 


\section{Syntheses of Cyclic Aminals}

\subsection{Solvent Screening}

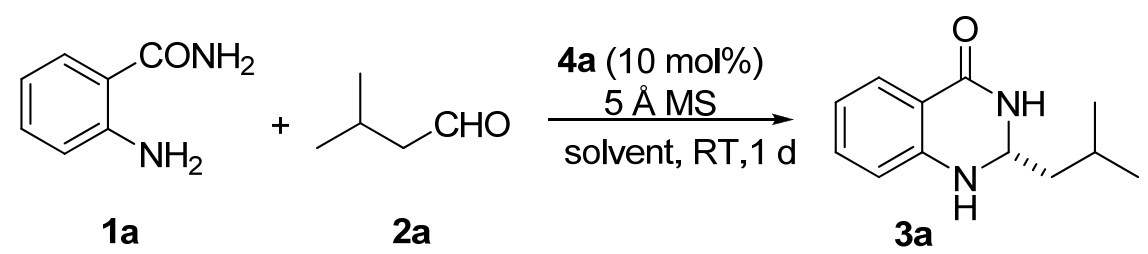

To a reaction vial charged with 2-aminobenzamide 1a (13.6 mg, $0.1 \mathrm{mmol})$, (S)-TRIP 4a (7.5 $\mathrm{mg}, 0.01 \mathrm{mmol})$ and $30 \mathrm{mg} 5 \AA \mathrm{MS}$ was added $1 \mathrm{~mL}$ solvent and 3-methylbutanal $2 \mathrm{a}$ (10.6 $\mu \mathrm{L}, 0.1 \mathrm{mmol}$ ) under argon. Then the mixture was stirred at room temperature for $24 \mathrm{~h}$. The reaction was monitored by TLC $(\mathrm{EtOH} / \mathrm{Hex}=1: 4, \mathrm{v} / \mathrm{v}) .0 .05 \mathrm{~mL}$ of the reaction mixture was taken and filtered through Celite with EA as eluent and subject to HPLC analysis. HPLC: $\mathrm{OD}-\mathrm{H}, \mathrm{Hept} /{ }^{i} \mathrm{PrOH}=80: 20, \mathrm{v} / \mathrm{v}, 220 \mathrm{~nm}, \mathrm{t}_{\text {minor }}=15.9 \mathrm{~min}, \mathrm{t}_{\text {major }}=19.9 \mathrm{~min}$.

\begin{tabular}{ccc}
\hline Entry & Solvent & Er \\
1 & Hex & $68: 32$ \\
2 & EA & $63: 37$ \\
3 & Toluene & $80: 20$ \\
4 & THF & $55: 45$ \\
5 & Dioxane & Racemic \\
6 & Ether & $60: 40$ \\
7 & DCM & $79: 21$ \\
8 & MeCN & $79: 21$ \\
9 & ${ }^{i} \operatorname{PrOH}$ & $64: 36$ \\
\hline
\end{tabular}

\subsection{Temperature Screening}

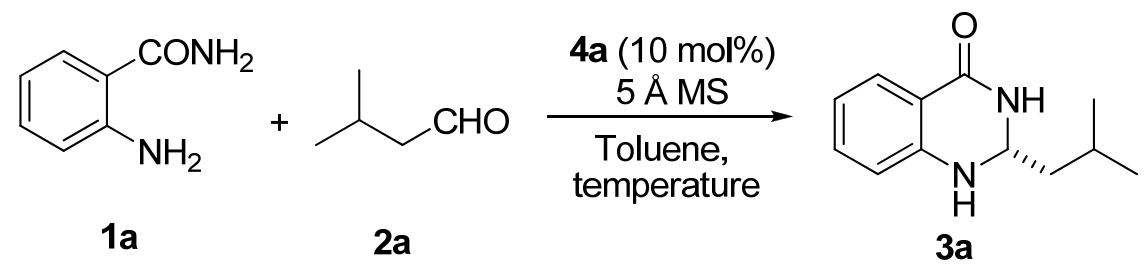

To a reaction vial charged with 2-aminobenzamide 1a (13.6 mg, $0.1 \mathrm{mmol})$, (S)-TRIP $4 a$ (7.5 $\mathrm{mg}, 0.01 \mathrm{mmol}$ ) and $30 \mathrm{mg} 5 \AA \mathrm{MS}$ was added $1 \mathrm{~mL}$ Toluene and put into the corresponding temperaute bath for 15 minutes, followed by the addtion of 3-methylbutanal $2 \mathrm{a}(10.6 \mu \mathrm{L}, 0.1$ mmol) under argon. Then the mixture was stirred at respective temperature for $24 \mathrm{~h}$. The reaction was monitored by TLC $(\mathrm{EtOH} / \mathrm{Hex}=1: 4, \mathrm{v} / \mathrm{v})$. The $0.05 \mathrm{~mL}$ of reaction mixture was taken and filtered through Celite with EA as eluent and subject to HPLC analysis. HPLC: OD-H, Hept $/{ }^{i} \mathrm{PrOH}=80: 20, \mathrm{v} / \mathrm{v}, 220 \mathrm{~nm}, \mathrm{t}_{\text {minor }}=15.9 \mathrm{~min}, \mathrm{t}_{\text {major }}=19.9 \mathrm{~min}$. 


\begin{tabular}{ccc}
\hline Entry & temperature & Er \\
1 & $\mathrm{RT}$ & $80: 20$ \\
2 & $-5{ }^{\circ} \mathrm{C}$ & $80: 20$ \\
3 & $-15{ }^{\circ} \mathrm{C}$ & $84: 16$ \\
4 & $-35{ }^{\circ} \mathrm{C}$ & $85: 15$ \\
5 & $-45^{\circ} \mathrm{C}$ & $86: 14$ \\
6 & $-45^{\circ} \mathrm{C}$ & $89: 11^{a}$ \\
7 & $-55^{\circ} \mathrm{C}$ & $83: 17$ \\
8 & $-78{ }^{\circ} \mathrm{C}$ & $75: 25$ \\
\hline
\end{tabular}

${ }^{a}$ The aldehyde was added as a solution in $1 \mathrm{~mL}$ Toluene during 4 hours.

\subsection{Catalyst Screening}

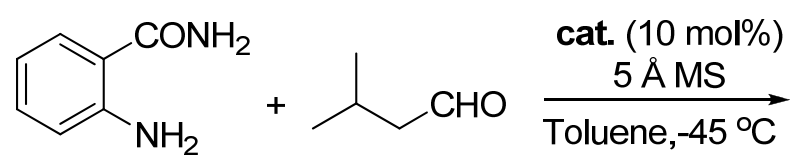

$1 \mathrm{a}$ 2a<smiles>CC(C)C[C@@H]1NC(=O)c2ccccc2N1</smiles>

3a

To a reaction vial charged with 2-aminobenzamide 1a (13.6 mg, $0.1 \mathrm{mmol})$, catalyst $(0.01$ mmol) and $30 \mathrm{mg} 5 \AA$ MS was added $1 \mathrm{~mL}$ Toluene and put into $-45^{\circ} \mathrm{C}$ bath for 15 minutes, followed by the addtion of 3-methylbutanal 2a $(10.6 \mu \mathrm{L}, 0.1 \mathrm{mmol})$ in $1 \mathrm{~mL}$ toluene during 4 hours under argon. Then the mixture was stirred at $-45{ }^{\circ} \mathrm{C}$ for $24 \mathrm{~h}$. The reaction was monitored by TLC $(\mathrm{EtOH} / \mathrm{Hex}=1: 4, \mathrm{v} / \mathrm{v})$. The $0.05 \mathrm{~mL}$ of reaction mixture was taken and filtered through Celite with EA as eluent and subject to HPLC analysis. HPLC: OD-H, $\mathrm{Hept} /{ }^{i} \mathrm{PrOH}=80: 20, \mathrm{v} / \mathrm{v}, 220 \mathrm{~nm}, \mathrm{t}_{\text {minor }}=15.9 \mathrm{~min}, \mathrm{t}_{\text {major }}=19.9 \mathrm{~min}$.<smiles>N#[W]c1cc2ccccc2c(-c2c(OP(=O)(O)O)c(Br)cc3ccccc23)c1OP(=O)(O)O</smiles><smiles>CCCc1cc(C(C)C)c(C(C)(C)C)c(C(C)C)c1</smiles><smiles>CC=Cc1ccccc1C(C)(C)C</smiles>

4b

er $80: 20$<smiles>CC(C)c1cc(C23CC4CC(CC(C4)C2)C3)cc(C(C)C)c1C(C)C</smiles>

4 e<smiles>O=P(O)(O)c1c(-c2ccccc2)c(-c2ccccc2)cc2ccc3ccccc3c12</smiles>

er 20:80<smiles>CC(C)c1cc(-c2c3ccccc3cc3ccccc23)cc(C(C)C)c1C(C)C</smiles>

4d er 99:1 er 96:4<smiles>FC(F)(F)c1cc(C(F)(F)F)cc(C(F)(F)F)c1</smiles>

$4 c$<smiles>Cc1ccc(-c2ccccc2)cc1</smiles>

er $58: 42$<smiles>Cc1cc(C)c(I)c(C)c1</smiles><smiles></smiles>

$\mathrm{SiPh}_{3}$<smiles>CC(C)(C)c1ccccc1</smiles>

er 68:32

er 58:42

er 79:21

er 58:42 


\subsection{Substrate Scope}

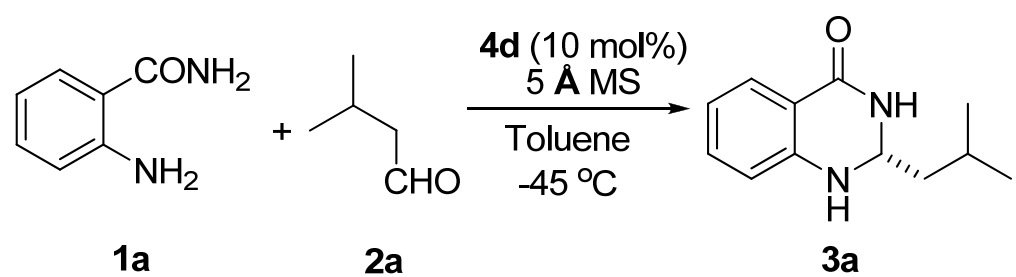

General Procedure: To a reaction vial charged with (S)-An-TRIP 4d (10.2 mg, $0.01 \mathrm{mmol})$, 2aminobenzamide 1a (13.6 mg, $0.1 \mathrm{mmol})$ and $30 \mathrm{mg} 5 \AA$ molecular sieves under argon was added $1 \mathrm{~mL}$ toluene and then cooled to $-45{ }^{\circ} \mathrm{C}$. A solution of 3-methylbutanal (10 $\mu \mathrm{L}, 0.1$ $\mathrm{mmol})$ in $1 \mathrm{~mL}$ toluene was added to the mixture during 4.5 hours $(0.1 \mathrm{~mL} / 30$ minutes $)$. Then the reaction mixture was stirred at $-45^{\circ} \mathrm{C}$ for 19.5 hours. A portion of reaction mixture was taken and pushed through a celite pad with ethyl acetate as eluent to give the HPLC analysis sample. The rest reaction mixture was concentrated to dryness and purified by silica gel chromatography with Hex/EtOH $(4: 1, \quad \mathrm{v} / \mathrm{v})$ as eluent to give (R)-2-isobutyl-2,3dihydroquinazolin-4(1H)-one $3 a(17.5 \mathrm{mg}, 86 \%)$ as white solid. ${ }^{1} \mathrm{H}$ NMR (500 $\mathrm{MHz}, \mathrm{CDCl}_{3}$ ) $\delta 0.99\left(\mathrm{~d}, J=6.5 \mathrm{~Hz}, \mathrm{CH}_{3}\right), 1.63-1.76\left(\mathrm{~m}, 2 \mathrm{H}, \mathrm{CH}_{2}\right), 1.76-1.83(\mathrm{~m}, 1 \mathrm{H}, \mathrm{MeCH}), 4.21$ (b, 1H, $\mathrm{NH}), 4.93$ (t, $J=6.2 \mathrm{~Hz}, 1 \mathrm{H}, \mathrm{NCH}), 6.20(\mathrm{~s}, 1 \mathrm{H}, \mathrm{NH}), 6.68$ (d, $J=8.1 \mathrm{~Hz}, 1 \mathrm{H}, \operatorname{ArH}), 6.87$ (t, $J=7.5 \mathrm{~Hz}, 1 \mathrm{H}, \mathrm{ArH}), 7.31(\mathrm{t}, J=8.0 \mathrm{~Hz}, 1 \mathrm{H}, \mathrm{ArH}), 7.89$ (d, $J=7.8 \mathrm{~Hz}, 1 \mathrm{H}, \mathrm{ArH}) .{ }^{13} \mathrm{C}$ NMR $\left(125 \mathrm{MHz}, \mathrm{CDCl}_{3}\right) \delta$ 22.6, 23.9, 44.4, 63.7, 114.9, 116.2, 119.5, 128.6, 133.8, 147.4, 165.3. MS (EI) m/z (\%) 204 (4), 147 (100). HRMS calculated for $\left(\mathrm{C}_{12} \mathrm{H}_{16} \mathrm{~N}_{2} \mathrm{O}\right)$ 204.126262, found 204.126460. HPLC, OD-H, Hept $/{ }^{i} \mathrm{PrOH}=80: 20,220 \mathrm{~nm}, \mathrm{t}_{\text {minor }}=15.9 \mathrm{~min}, \mathrm{t}_{\text {major }}=19.9 \mathrm{~min}$.<smiles>CCC[C@H]1NC(=O)c2ccccc2N1</smiles>

(R)-2-Propyl-2,3-dihydroquinazolin-4(1H)-one $\mathbf{3 b}$ was prepared by the same method as that for 3a from 2-aminobenzamide (13.6 mg, $0.1 \mathrm{mmol}$ ) and butyraldehyde $\mathbf{2 b}$ (9 $\mu \mathrm{L}, 0.1 \mathrm{mmol}$ ) as white solid (17.3 mg, 91\%). ${ }^{1} \mathrm{H}$ NMR (500 MHz, $\left.\mathrm{CDCl}_{3}\right) \delta 1.00\left(\mathrm{t}, J=7.4 \mathrm{~Hz}, 3 \mathrm{H}, \mathrm{CH}_{3}\right.$ ), 1.44-1.54 (m, 2H, $\left.\mathrm{CH}_{2}\right), 1.74-1.79\left(\mathrm{~m}, 2 \mathrm{H}, \mathrm{CH}_{2}\right), 3.26$ (b, $\left.1 \mathrm{H}, \mathrm{NH}\right), 4.89(\mathrm{t}, J=5.8 \mathrm{~Hz}, 1 \mathrm{H}$, $\mathrm{NCH}$ ), 6.29 (b, 1H, NH), 6.67 (d, $J=8.0 \mathrm{~Hz}, 1 \mathrm{H}), 6.85$ (t, $J=7.5 \mathrm{~Hz}, 1 \mathrm{H}, \mathrm{ArH}), 7.30$ (t, $J=$ $6.9 \mathrm{~Hz}, 1 \mathrm{H}, \mathrm{ArH}), 7.98$ (d, $J=7.8 \mathrm{~Hz}, 1 \mathrm{H}, \mathrm{ArH}) .{ }^{13} \mathrm{C}$ NMR $\left(75 \mathrm{MHz}, \mathrm{CDCl}_{3}\right) \delta$ 13.8, 17.4, 37.7, 65.1, 114.7, 116.0, 119.4, 128.6, 133.8, 147.4, 165.3. MS (EI) m/z (\%) 190 (5), 147 (100). HRMS calculated for $\left(\mathrm{C}_{11} \mathrm{H}_{14} \mathrm{~N}_{2} \mathrm{O}\right)$ 190.110610, found 190.110533. HPLC, OD-H, $\mathrm{Hept} /{ }^{i} \mathrm{PrOH}=80: 20,220 \mathrm{~nm}, \mathrm{t}_{\text {minor }}=15.7 \mathrm{~min}, \mathrm{t}_{\text {marjor }}=18.7 \mathrm{~min}$.

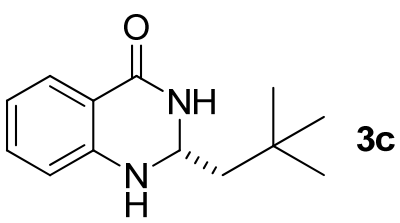

(R)-2-Neopentyl-2,3-dihydroquinazolin-4(1H)-one 3c was prepared by the same method as that for 3a from 2-aminobenzamide (13.6 mg, $0.1 \mathrm{mmol})$ and 3,3-dimethylbutanal 2c $(12 \mu \mathrm{L}$, $0.1 \mathrm{mmol}$ ) as white solid (20.5 mg, 94\%). $\left.{ }^{1} \mathrm{H} \mathrm{NMR} \mathrm{(400} \mathrm{MHz,} \mathrm{CDCl}_{3}\right) \delta 1.02\left(\mathrm{~s}, 9 \mathrm{H}, \mathrm{CH}_{3}\right)$, 1.72 (d, $J=5.2 \mathrm{~Hz}, 2 \mathrm{H}, \mathrm{CH}_{2}$ ), 4.21 (s, $\left.1 \mathrm{H}, \mathrm{NH}\right), 4.95$ (t, $\left.J=5.2 \mathrm{~Hz}, 1 \mathrm{H}, \mathrm{NCH}\right), 6.17$ (b, 1H, $\mathrm{NH}), 6.68(\mathrm{~d}, J=8.0 \mathrm{~Hz}, 1 \mathrm{H}), 6.87(\mathrm{t}, J=7.5 \mathrm{~Hz}, 1 \mathrm{H}, \mathrm{ArH}), 7.30(\mathrm{t}, J=8.0 \mathrm{~Hz}, 1 \mathrm{H}, \operatorname{ArH})$, 7.89 (d, $J=7.8 \mathrm{~Hz}, 1 \mathrm{H}, \mathrm{ArH}) .{ }^{13} \mathrm{C}$ NMR $\left(100 \mathrm{MHz}, \mathrm{CDCl}_{3}\right) \delta 30.0,50.0,63.4,115.0,116.3$, 
119.5, 128.6, 133.7, 147.5, 165.2. MS (EI) m/z (\%) 218 (3), 147 (100). HRMS calculated for $\left(\mathrm{C}_{13} \mathrm{H}_{18} \mathrm{~N}_{2} \mathrm{O}\right)$ 218.141916, found 218.141802. HPLC, OD-H, Hept ${ }^{i} \mathrm{PrOH}=80: 20,220 \mathrm{~nm}$, $\mathrm{t}_{\mathrm{minor}}=17.8 \mathrm{~min}, \mathrm{t}_{\mathrm{marjor}}=20.3 \mathrm{~min}$.<smiles>O=C1N[C@@H](CC2CCCCC2)Nc2ccccc21</smiles>

(R)-2-(Cyclohexylmethyl)-2,3-dihydroquinazolin-4(1H)-one 3d was prepared by the same method as that for 3a from 2-aminobenzamide (13.6 $\mathrm{mg}, 0.1 \mathrm{mmol})$ and 2cyclohexylacetaldehyde $2 \mathbf{d}(16 \mu \mathrm{L}, 0.1 \mathrm{mmol})$ as white solid $(20.5 \mathrm{mg}, 84 \%) .{ }^{1} \mathrm{H}$ NMR (500 $\left.\mathrm{MHz} \mathrm{CDCl}_{3}\right) \delta$ 0.95-1.02 (m, 2H, H from Cy), 1.13-1.31(m, 3H, H from Cy), 1.44-1.48 (m, 1H, CH from Cy), 1.64-1.76 (m, 7H, H from Cy), $4.17(\mathrm{~s}, 1 \mathrm{H}, \mathrm{NH}), 4.97(\mathrm{t}, J=5.2 \mathrm{~Hz}, 1 \mathrm{H}$, $\mathrm{NCH}), 5.88(\mathrm{~s}, 1 \mathrm{H}, \mathrm{NH}), 6.68(\mathrm{~d}, J=8.0 \mathrm{~Hz}, 1 \mathrm{H}), 6.87$ (t, $J=7.5 \mathrm{~Hz}, 1 \mathrm{H}, \operatorname{ArH}), 7.30$ (t, $J=$ $7.5 \mathrm{~Hz}, 1 \mathrm{H}, \mathrm{ArH}), 7.89$ (d, $J=7.8 \mathrm{~Hz}, 1 \mathrm{H}, \mathrm{ArH}) .{ }^{13} \mathrm{C}$ NMR $\left(125 \mathrm{MHz}, \mathrm{CDCl}_{3}\right) \delta 26.0,26.1$, 26.2, 33.3, 33.4, 43.1, 63.1, 114.9, 116.2, 119.5, 128.6, 133.7, 147.5, 165.3. MS (EI) m/z (\%) 244 (2), 147 (100). HRMS calculated for $\left(\mathrm{C}_{15} \mathrm{H}_{20} \mathrm{~N}_{2} \mathrm{ONa}\right)$ 267.146783, found 267.146569. HPLC, OD-H, Hept ${ }^{i} \mathrm{PrOH}=80: 20,254 \mathrm{~nm}, \mathrm{t}_{\text {minor }}=16.8 \mathrm{~min}, \mathrm{t}_{\text {marjor }}=19.2 \mathrm{~min}$.<smiles>O=C1N[C@@H](Cc2ccccc2)Nc2ccccc21</smiles>

$(R)$ - 2-Benzyl-2,3-dihydroquinazolin-4(1H)-one 3e was prepared by the same method as that for 3a from 2-aminobenzamide (13.6 mg, $0.1 \mathrm{mmol}$ ) and 2-phenylacetaldehyde $2 \mathbf{e}(15 \mu \mathrm{L}, 0.1$ mmol) as white solid (19.0 mg, 80\%). ${ }^{1} \mathrm{H}$ NMR (500 MHz, $\left.\mathrm{CDCl}_{3}\right) \delta 3.02-3.12\left(\mathrm{~m}, 2 \mathrm{H}, \mathrm{CH}_{2}\right)$, 5.04 (t, $J=7.0 \mathrm{~Hz}, 1 \mathrm{H}, \mathrm{NCH}), 6.18$ (s, 1H, NH), 6.61 (d, $J=8.0 \mathrm{~Hz}, 1 \mathrm{H}, \mathrm{ArH}), 6.86$ (t, $J=7.5$ Hz, 1H, ArH), 7.22 (d, $J=7.1 \mathrm{~Hz}, 2 \mathrm{H}, \mathrm{ArH}), 7.29-7.33$ (m, 2H, ArH), 7.38 (t, $J=7.5 \mathrm{~Hz}, 2 \mathrm{H}$, ArH), 7.89 (d, $J=7.8 \mathrm{~Hz}, 1 \mathrm{H}, \mathrm{ArH}) .{ }^{13} \mathrm{C}$ NMR (125 MHz, $\left.\mathrm{CDCl}_{3}\right) \delta 16.9,17.0,32.8,70.2$, 114.5, 115.5, 119.1, 128.5, 133.9, 147.4, 165.2. MS (EI) m/z (\%) 237 (0.35), 147 (100). HRMS calculated for $\left(\mathrm{C}_{15} \mathrm{H}_{14} \mathrm{~N}_{2} \mathrm{ONa}\right)$ 261.099834, found 261.099689. HPLC, OD-H, H $\mathrm{ept} /{ }^{i} \mathrm{PrOH}=80: 20,220 \mathrm{~nm}, \mathrm{t}_{\mathrm{major}}=26.5 \mathrm{~min}, \mathrm{t}_{\mathrm{minor}}=32.4 \mathrm{~min}$.<smiles>CC(Br)[C@@H]1NC(=O)c2ccccc2N1</smiles>

(R)-2-Isopropyl-2,3-dihydroquinazolin-4(1H)-one $3 \mathbf{f}$ was prepared by the same method as that for 3a from 2-aminobenzamide (13.6 $\mathrm{mg}, 0.1 \mathrm{mmol}$ ) and isobutyraldehyde $2 \mathbf{f}$ ( $9 \mu \mathrm{L}, 0.1$ mmol) as white solid (13.7 mg, 72\%). ${ }^{1} \mathrm{H}$ NMR (500 MHz, $\left.\mathrm{CDCl}_{3}\right) \delta 1.06(\mathrm{t}, J=6.8 \mathrm{~Hz}, 6 \mathrm{H}$, $\left.\mathrm{CH}_{3}\right), 1.92-2.01\left(\mathrm{~m}, 1 \mathrm{H}, \mathrm{Me}_{2} \mathrm{CH}\right), 4.70$ (d, $\left.J=4.8 \mathrm{~Hz}, 1 \mathrm{H}, \mathrm{NCH}\right), 6.01$ (s, $\left.1 \mathrm{H}, \mathrm{NH}\right), 6.66$ (d, $J$ $=8.1 \mathrm{~Hz}, 1 \mathrm{H}, \mathrm{ArH}), 6.83(\mathrm{t}, J=7.7 \mathrm{~Hz}, 1 \mathrm{H}, \operatorname{ArH}), 7.30$ (t, $J=7.7 \mathrm{~Hz}, 1 \mathrm{H}, \operatorname{ArH}), 7.88$ (d, $J=$ $7.8 \mathrm{~Hz}, 1 \mathrm{H}, \mathrm{ArH}) .{ }^{13} \mathrm{C}$ NMR $\left(125 \mathrm{MHz}, \mathrm{CDCl}_{3}\right) \delta 16.9,17.0,32.8,70.2,114.5,115.5,119.1$, 128.5, 133.9, 147.4, 165.2. MS (EI) m/z (\%) 190 (3), 147 (100), 92 (7). HRMS calculated for $\left(\mathrm{C}_{11} \mathrm{H}_{14} \mathrm{~N}_{2} \mathrm{O}\right)$ 190.110615, found 190.110477. HPLC, OD-H, Hept ${ }^{i} \mathrm{PrOH}=80: 20,220 \mathrm{~nm}$, $\mathrm{t}_{\text {minor }}=13.3 \mathrm{~min}, \mathrm{t}_{\mathrm{major}}=19.5 \mathrm{~min}$. 
<smiles>O=C1N[C@@H](c2ccccc2)Nc2ccccc21</smiles>

(R)-2-Phenyl-2,3-dihydroquinazolin-4(1H)-one $3 \mathbf{g}$ was prepared by the same method as that for 3a except at $-15{ }^{\circ} \mathrm{C}$ from 2-aminobenzamide (13.6 $\mathrm{mg}, 0.1 \mathrm{mmol}$ ) and benzaldehyde $\mathbf{2 g}$ $(10 \mu \mathrm{L}, 0.1 \mathrm{mmol})$ as white solid (15.0 mg, 67\%). ${ }^{1} \mathrm{H} \mathrm{NMR}\left(500 \mathrm{MHz}, \mathrm{CDCl}_{3}\right) \delta 4.38(\mathrm{~b}, 1 \mathrm{H}$, $\mathrm{NH}), 5.77$ (s, 1H, NH), 5.91 (s, 1H, NCH), 6.01 (s, 1H, NH), 6.67 (d, $J=8.0 \mathrm{~Hz}, 1 \mathrm{H}, \operatorname{ArH})$, 6.91 (t, $J=7.8 \mathrm{~Hz}, 1 \mathrm{H}, \mathrm{ArH}), 7.34$ (t, $J=8.0 \mathrm{~Hz}, 1 \mathrm{H}, \mathrm{ArH}), 7.45-7.47$ (m, 3H, ArH), 7.507.62 (m, 2H, ArH), 7.95 (d, $J=7.8 \mathrm{~Hz}, 1 \mathrm{H}, \mathrm{ArH}) .{ }^{13} \mathrm{C}$ NMR (125 MHz, $\left.\mathrm{CDCl}_{3}\right) \delta$ 69.1, 114.6, 115.6, 119.7, 127.4, 128.8, 129.2, 130.2, 134.1, 138.5, 147.2, 164.7. MS (EI) m/z (\%) 224 (24), 147 (100), 120 (41), 92 (14). HRMS calculated for $\left(\mathrm{C}_{14} \mathrm{H}_{12} \mathrm{~N}_{2} \mathrm{O}\right)$ 224.094959, found 224.094761. HPLC, OD-H, Hept $/{ }^{i} \mathrm{PrOH}=80: 20,254 \mathrm{~nm}, \mathrm{t}_{\text {minor }}=25.7 \mathrm{~min}, \mathrm{t}_{\text {major }}=28.5 \mathrm{~min}$.

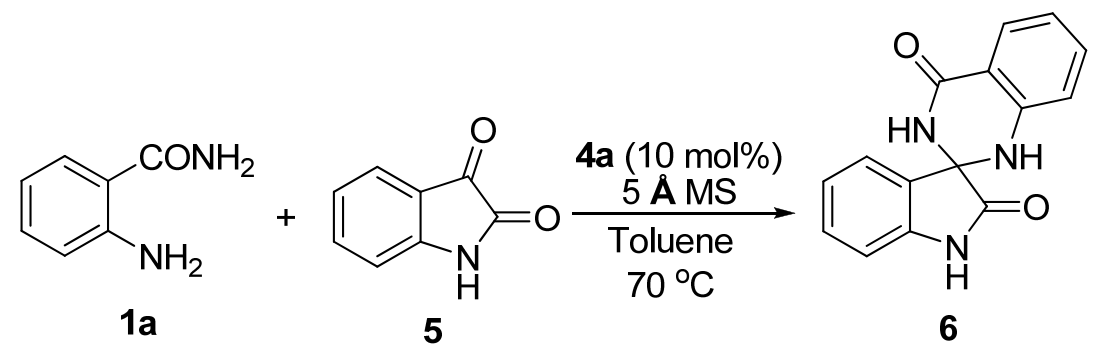

To a reaction vial charged with (S)-TRIP 4a (7.5 $\mathrm{mg}, 0.01 \mathrm{mmol})$, 2-aminobenzamide 1a (13.6 mg, $0.1 \mathrm{mmol})$, Isatin 5 (14.7 mg, $1 \mathrm{mmol}$ ) and $30 \mathrm{mg} 5 \AA$ molecular sieves under argon was added $2 \mathrm{~mL}$ toluene and then the reaction mixture was stirred at $70{ }^{\circ} \mathrm{C}$ for 5 hours. A portion of reaction mixture was taken and pushed through a celite pad with ethyl acetate as eluent to give the HPLC analysis sample. The rest reaction mixture was concentrated to dryness and purified by silica gel chromatography with Hex/EA (1:3, v/v) as eluent to give 1'H-spiro[indoline-3,2'-quinazoline]-2,4'(3'H)-dione 6 as white solid (22.5 mg, 85\%). ${ }^{1} \mathrm{H}$ NMR (500 MHz, CD $\left.{ }_{3} \mathrm{OD}\right) \delta 6.54(\mathrm{~d}, J=8.0 \mathrm{~Hz}, 1 \mathrm{H}, \operatorname{ArH}), 6.65(\mathrm{t}, \quad J=7.0 \mathrm{~Hz}, 1 \mathrm{H}, \operatorname{ArH})$, $6.80(\mathrm{~d}, J=8.1 \mathrm{~Hz}, 1 \mathrm{H}, \operatorname{ArH}), 7.01(\mathrm{t}, J=7.5 \mathrm{~Hz}, 1 \mathrm{H}, \operatorname{ArH}), 7.16(\mathrm{t}, J=8.0 \mathrm{~Hz}, 1 \mathrm{H}, \operatorname{ArH})$, $7.24(\mathrm{t}, J=8.0 \mathrm{~Hz}, 1 \mathrm{H}, \operatorname{ArH}), 7.46(\mathrm{~d}, J=7.5 \mathrm{~Hz}, 1 \mathrm{H}, \operatorname{ArH}), 7.62(\mathrm{~d}, J=7.5 \mathrm{~Hz}, 1 \mathrm{H}$, $\mathrm{ArH}) .{ }^{13} \mathrm{C}$ NMR (125 MHz, $\left.\mathrm{CD}_{3} \mathrm{OD}\right) \delta 111.6,115.5,119.2,121.3,124.2,124.6,126.5,128.4$, 130.3, 130.5, 132.3, 135.2, 143.2, 148.4, 167.6, 178.3. MS (EI) m/z (\%) 265 (2), 237 (100), 119 (68), 92 (19).HRMS(ESI) calculated for $\left(\mathrm{C}_{15} \mathrm{H}_{11} \mathrm{~N}_{3} \mathrm{O}_{2} \mathrm{Na}\right) 288.074347$, found 288.074271. HPLC, IA, Hept ${ }^{i} \mathrm{PrOH}=80: 20,220 \mathrm{~nm}, \mathrm{t}_{\text {minor }}=23.9 \mathrm{~min}, \mathrm{t}_{\text {major }}=29.3 \mathrm{~min}$.<smiles>Cc1ccc2c(c1)N[C@@H](CC(C)C)NC2=O</smiles>

(R)-2-Isobutyl-7-methyl-2,3-dihydroquinazolin-4(1H)-one $3 \mathbf{h}$ was prepared by the same method as that for 3a from 2-amino-4-methylbenzamide $\mathbf{1 b}(15.0 \mathrm{mg}, 0.1 \mathrm{mmol})$ and 3methylbutanal (10 $\mu \mathrm{L}, 0.1 \mathrm{mmol})$ as white solid (20.1 mg, 84\%). ${ }^{1} \mathrm{H}$ NMR (500 $\mathrm{MHz}, \mathrm{CDCl}_{3}$ ) $\delta 0.99$ (d, $\left.J=6.5 \mathrm{~Hz}, 6 \mathrm{H}, \mathrm{CH}_{3}\right), 1.66$ (t, $\left.J=7.2 \mathrm{~Hz}, 2 \mathrm{H}, \mathrm{CH}_{2}\right), 1.72-1.83$ (m, $1 \mathrm{H}, \mathrm{Me}_{2} \mathrm{CH}$ ), 2.30 (s, 3H, $\left.\mathrm{ArCH}_{3}\right), 4.91$ (t, $\left.J=6.2 \mathrm{~Hz}, 1 \mathrm{H}, \mathrm{NCH}\right), 5.82$ (s, 1H, NH), 6.49 (s, 1H, ArH), 6.69 (d, $J=8.0 \mathrm{~Hz}, 1 \mathrm{H}, \mathrm{ArH}), 7.78$ (d, $\mathrm{J}=8.0 \mathrm{~Hz}, 1 \mathrm{H}, \mathrm{ArH}) .{ }^{13} \mathrm{C} \mathrm{NMR}\left(125 \mathrm{MHz}, \mathrm{CDCl}_{3}\right) \delta 21.8$, 22.6, 22.7, 23.9, 44.4, 63.7, 115.2, 120.9, 128.6, 144.7, 147.4, 165.3. MS (EI) m/z (\%) 218 (4), 
161 (100). HRMS calculated for $\left(\mathrm{C}_{13} \mathrm{H}_{18} \mathrm{~N}_{2} \mathrm{O}\right)$ 218.141914, found 218.142074. HPLC, OD-H, $\mathrm{Hept} /{ }^{i} \mathrm{PrOH}=80: 20,254 \mathrm{~nm}, \mathrm{t}_{\text {minor }}=15.6 \mathrm{~min}, \mathrm{t}_{\text {major }}=19.6 \mathrm{~min}$.<smiles>Cc1ccc2c(c1)C(=O)N[C@@H](CC(C)C)N2</smiles>

(R)-2-Isobutyl-6-methyl-2,3-dihydroquinazolin-4(1H)-one $3 \mathbf{i}$ was prepared by the same method as that for 3a from 2-amino-5-methylbenzamide 1c (15 mg, $0.1 \mathrm{mmol})$ and butyraldehyde (10 $\mu \mathrm{L}, 0.1 \mathrm{mmol}$ ) as white solid (20.0 mg, 84\%). ${ }^{1} \mathrm{H}$ NMR (500 $\mathrm{MHz}, \mathrm{CDCl}_{3}$ ) $\delta 0.91\left(\mathrm{~d}, J=2.5 \mathrm{~Hz}, 3 \mathrm{H}, \mathrm{CH}_{3}\right), 0.92\left(\mathrm{~d}, J=2.5 \mathrm{~Hz}, 3 \mathrm{H}, \mathrm{CH}_{3}\right), 1.57-1.60$ (m, $\left.2 \mathrm{H}, \mathrm{CH}_{2}\right), 1.68-$ $1.76(\mathrm{~m}, 1 \mathrm{H}, \mathrm{MeCH}), 2.21\left(\mathrm{~s}, 3 \mathrm{H}, \mathrm{CH}_{3}\right), 4.81(\mathrm{t}, J=6.2 \mathrm{~Hz}, 1 \mathrm{H}, \mathrm{NCH}), 5.87$ (s, $\left.1 \mathrm{H}, \mathrm{NH}\right), 6.54$ (d, $J=8.7 \mathrm{~Hz}, 1 \mathrm{H}, \mathrm{ArH}), 7.05-7.07$ (m, $1 \mathrm{H}, \mathrm{ArH}), 7.63$ (s, $1 \mathrm{H}, \mathrm{ArH}) .{ }^{13} \mathrm{C}$ NMR $(125 \mathrm{MHz}$, $\left.\mathrm{CDCl}_{3}\right) \delta 20.4,22.2,23.9,44.3,63.7,115.1,116.3,128.5,129.1,134.7,145.1,165.8 . \mathrm{MS}(\mathrm{EI})$ $\mathrm{m} / \mathrm{z}$ (\%) 218 (5), 161 (100). HRMS(ESI) calculated for $\left(\mathrm{C}_{13} \mathrm{H}_{15} \mathrm{~N}_{2} \mathrm{ONa}\right)$ 241.131130, found 241.131061. HPLC, OD-H, Hept $/{ }^{i} \mathrm{PrOH}=80: 20,220 \mathrm{~nm}, \mathrm{t}_{\text {minor }}=15.2 \mathrm{~min}, \mathrm{t}_{\text {major }}=20.1 \mathrm{~min}$.<smiles>Cc1cccc2c1C(=O)N[C@@H](CC(C)C)N2</smiles>

(R)-2-Isobutyl-5-methyl-2,3-dihydroquinazolin-4(1H)-one $3 \mathbf{j}$ was prepared by the same method as that for 3a from 2-amino-6-methylbenzamide 1d (15.0 mg, $0.1 \mathrm{mmol})$ and 3methylbutanal (10 $\mu \mathrm{L}, 0.1 \mathrm{mmol})$ as white solid (18.1 mg, 83\%). ${ }^{1} \mathrm{H}$ NMR (500 MHz, $\mathrm{CDCl}_{3}$ ) $\delta 0.98\left(\mathrm{~d}, J=6.5 \mathrm{~Hz}, 3 \mathrm{H}, \mathrm{CH}_{3}\right), 0.99$ (d, $\left.J=6.5 \mathrm{~Hz}, 3 \mathrm{H}, \mathrm{CH}_{3}\right), 1.66\left(\mathrm{t}, J=7.2 \mathrm{~Hz}, 2 \mathrm{H}, \mathrm{CH}_{2}\right)$, 1.76-1.85 (m, 1H, Me $\mathrm{CH}_{2}$ ), 2.67 (s, 3H, $\left.\mathrm{ArCH}_{3}\right), 4.11$ (b, $\left.1 \mathrm{H}, \mathrm{NH}\right), 4.81$ (t, J = 7.5 Hz, 1H, $\mathrm{NCH}$ ), 6.05 (s, 1H, NH), 6.54 (d, $J=8.0 \mathrm{~Hz}, 1 \mathrm{H}, \operatorname{ArH}), 6.67$ (d, $J=7.5 \mathrm{~Hz}, 1 \mathrm{H}, \operatorname{ArH}), 7.14$ (t, $J=7.8 \mathrm{~Hz}, 1 \mathrm{H}, \mathrm{ArH}) .{ }^{13} \mathrm{C} \mathrm{NMR}\left(125 \mathrm{MHz}, \mathrm{CDCl}_{3}\right) \delta 22.1,22.5,22.7,23.9,44.1,63.0,113.1$, 115.0, 123.3, 132.5, 142.4, 148.9, 165.8. MS (EI) m/z (\%) 218 (4), 161 (100). HRMS calculated for $\left(\mathrm{C}_{13} \mathrm{H}_{18} \mathrm{~N}_{2} \mathrm{O}\right)$ 218.141909, found 218.141848. HPLC, OD-H, Hept $/{ }^{i} \mathrm{PrOH}=$ $80: 20,254 \mathrm{~nm}, \mathrm{t}_{\mathrm{minor}}=14.0 \mathrm{~min}, \mathrm{t}_{\mathrm{major}}=15.8 \mathrm{~min}$.<smiles>CC(C)C[C@@H]1NC(=O)c2cc(Cl)ccc2N1</smiles>

(R)-6-Chloro-2-isobutyl-2,3-dihydroquinazolin-4(1H)-one $3 \mathbf{k}$ was prepared by the same method as that for 3a from 2-amino-5-chlorobenzamide 1e $(17.0 \mathrm{mg}, 0.1 \mathrm{mmol})$ and 3methylbutanal (10 $\mu \mathrm{L}, 0.1 \mathrm{mmol}$ ) as white solid (21.4 mg, 90\%). ${ }^{1} \mathrm{H}$ NMR (500 $\mathrm{MHz}, \mathrm{CDCl}_{3}$ ) $\delta 0.98$ (d, $J=6.5 \mathrm{~Hz}, 6 \mathrm{H}, \mathrm{CH}_{3}$ ), 1.63-1.72 (m, 2H, $\mathrm{CH}_{2}$ ), 1.76-1.84 (m, 1H, $\mathrm{Me}_{2} \mathrm{CH}$ ), 4.29 (s, $1 \mathrm{H}, \mathrm{NH}), 4.91$ (t, $J=6.3 \mathrm{~Hz}, 1 \mathrm{H}, \mathrm{NCH}), 6.63(\mathrm{~d}, J=8.5 \mathrm{~Hz}, 1 \mathrm{H}, \mathrm{ArH}), 6.73$ (s, $1 \mathrm{H}, \mathrm{NH}), 7.24$ (dd, $J=2.5$ and $8.5 \mathrm{~Hz}, 1 \mathrm{H}, \mathrm{ArH}), 7.84(\mathrm{~d}, J=2.5 \mathrm{~Hz}, 1 \mathrm{H}, \mathrm{ArH}) .{ }^{13} \mathrm{C}$ NMR $\left(125 \mathrm{MHz}, \mathrm{CDCl}_{3}\right)$ $\delta$ 22.6, 24.0, 44.3, 63.6, 116.4, 124.6, 128.2, 133.7, 145.8, 164.1. MS (EI) m/z (\%) 238 (5), 183 (31), 181 (100). HRMS calculated for $\left(\mathrm{C}_{12} \mathrm{H}_{15} \mathrm{~N}_{2} \mathrm{OClNa}\right) 261.076509$, found 261.076150. HPLC, OD-H, Hept ${ }^{i} \mathrm{PrOH}=80: 20,254 \mathrm{~nm}, \mathrm{t}_{\mathrm{minor}}=10.6 \mathrm{~min}, \mathrm{t}_{\mathrm{major}}=13.6 \mathrm{~min}$. 
<smiles>CC(C)C[C@@H]1NC(=O)c2c(Cl)cccc2N1</smiles>

(R)-5-Chloro-2-isobutyl-2,3-dihydroquinazolin-4(1H)-one 31 was prepared by the same method as that for 3a from 2-amino-6-chlorobenzamide 1f $(17.0 \mathrm{mg}, 0.1 \mathrm{mmol})$ and 3methylbutanal $(10 \mu \mathrm{L}, 0.1 \mathrm{mmol})$ as white solid $(19.5 \mathrm{mg}, 82 \%) .{ }^{1} \mathrm{H}$ NMR (500 $\mathrm{MHz}, \mathrm{CDCl}_{3}$ ) $\delta 0.98\left(\mathrm{~d}, J=6.5 \mathrm{~Hz}, 3 \mathrm{H}, \mathrm{CH}_{3}\right), 0.99\left(\mathrm{~d}, J=6.5 \mathrm{~Hz}, 3 \mathrm{H}, \mathrm{CH}_{3}\right), 1.59-1.72\left(\mathrm{~m}, 2 \mathrm{H}, \mathrm{CH}_{2}\right), 1.76-$ $1.84\left(\mathrm{~m}, 1 \mathrm{H}, \mathrm{Me}_{2} \mathrm{CH}\right), 4.81(\mathrm{t}, J=6.0 \mathrm{~Hz}, 1 \mathrm{H}, \mathrm{NCH}), 6.28(\mathrm{~s}, 1 \mathrm{H}, \mathrm{NH}), 6.60(\mathrm{~d}, J=8.0 \mathrm{~Hz}$, $1 \mathrm{H}, \mathrm{ArH}), 6.88(\mathrm{~d}, J=8.0 \mathrm{~Hz}, 1 \mathrm{H}, \mathrm{ArH}), 7.14(\mathrm{t}, J=8.0 \mathrm{~Hz}, 1 \mathrm{H}, \mathrm{ArH}) .{ }^{13} \mathrm{C}$ NMR $(125 \mathrm{MHz}$, $\left.\mathrm{CDCl}_{3}\right) \delta 22.4,22.7,23.9,43.7,62.8,114.0,122.8,134.0,135.9,150.1,162.9 . \mathrm{MS}(\mathrm{EI}) \mathrm{m} / \mathrm{z}$ (\%) 238 (3), 183 (32), 181 (100). HRMS(ESI) calculated for $\left(\mathrm{C}_{12} \mathrm{H}_{15} \mathrm{~N}_{2} \mathrm{OCINa}\right) 261.076509$, found 261.076258. HPLC, OD-H, Hept $/{ }^{i} \mathrm{PrOH}=80: 20,220 \mathrm{~nm}, \mathrm{t}_{\text {minor }}=15.8 \mathrm{~min}, \mathrm{t}_{\text {major }}=18.1$ min.<smiles>CC(C)C[C@@H]1NC(=O)c2cc(Br)ccc2N1</smiles>

(R)-5-Bromo-2-isobutyl-2,3-dihydroquinazolin-4(1H)-one $3 \mathbf{m}$ was prepared by the same method as that for 3a from 2-amino-5-bromobenzamide $\mathbf{1 g}(21.3 \mathrm{mg}, 0.1 \mathrm{mmol})$ and butyraldehyde (10 $\mu \mathrm{L}, 0.1 \mathrm{mmol}$ ) as white solid (24.0 mg, 85\%). ${ }^{1} \mathrm{H}$ NMR (500 MHz, $\mathrm{CDCl}_{3}$ ) $\delta 0.99\left(\mathrm{~d}, J=2.5 \mathrm{~Hz}, 3 \mathrm{H}, \mathrm{CH}_{3}\right), 1.00\left(\mathrm{~d}, J=2.5 \mathrm{~Hz}, 3 \mathrm{H}, \mathrm{CH}_{3}\right), 1.66-1.70\left(\mathrm{~m}, 2 \mathrm{H}, \mathrm{CH}_{2}\right), 1.75-$ $1.82(\mathrm{~m}, 1 \mathrm{H}, \mathrm{MeCH}), 4.93(\mathrm{t}, J=6.2 \mathrm{~Hz}, 1 \mathrm{H}, \mathrm{NCH}), 6.23(\mathrm{~s}, 1 \mathrm{H}, \mathrm{NH}), 6.59(\mathrm{~d}, J=8.7 \mathrm{~Hz}, 1 \mathrm{H}$, ArH), 7.38-7.40 (m, 1H, Ar-H), 8.01 (s, $1 \mathrm{H}, \mathrm{ArH}) .{ }^{13} \mathrm{C}$ NMR (125 MHz, $\left.\mathrm{CDCl}_{3}\right) \delta 25.9,26.2$, 27.4, 48.0, 67.2, 114.4, 120.5, 134.4, 140.4, 142.2, 150.6, 168.6. MS (EI) m/z (\%) 284 (6), 282 (6), 227 (97), 225 (100). HRMS(ESI) calculated for $\left(\mathrm{C}_{12} \mathrm{H}_{15} \mathrm{BrN}_{2} \mathrm{ONa}\right)$ 305.026003, found 305.026026. HPLC, OD-H, Hept $/{ }^{i} \mathrm{PrOH}=80: 20,220 \mathrm{~nm}, \mathrm{t}_{\text {minor }}=11.0 \mathrm{~min}, \mathrm{t}_{\text {major }}=14.3$ min.<smiles>CC(C)C[C@@H]1NC(=O)c2c(F)cccc2N1</smiles>

(R)-5-Fluoro-2-isobutyl-2,3-dihydroquinazolin-4(1H)-one $3 \mathbf{n}$ was prepared by the same method as that for 3a from 2-amino-6-fluorobenzamide $\mathbf{1 h}(15.4 \mathrm{mg}, 0.1 \mathrm{mmol})$ and 3methylbutanal $(10 \mu \mathrm{L}, 0.1 \mathrm{mmol})$ as white solid (20.0 mg, 90\%). ${ }^{1} \mathrm{H}$ NMR (500 MHz, $\mathrm{CDCl}_{3}$ ) $\delta 0.98\left(\mathrm{~d}, J=6.5 \mathrm{~Hz}, 3 \mathrm{H}, \mathrm{CH}_{3}\right), 0.99(\mathrm{~d}, J=6.5 \mathrm{~Hz}, 3 \mathrm{H}, \mathrm{ArH}), 1.61-1.71\left(\mathrm{~m}, 2 \mathrm{H}, \mathrm{CH}_{2}\right), 1.74-$ $1.82(\mathrm{~m}, 1 \mathrm{H}, \mathrm{MeCH}), 4.96$ (t, $J=6.3 \mathrm{~Hz}, 1 \mathrm{H}, \mathrm{NCH}), 6.04(\mathrm{~s}, 1 \mathrm{H}, \mathrm{NH}), 6.47$ (d, $J=8.1 \mathrm{~Hz}, 1 \mathrm{H}$, ArH), 6.53 (dd, $J=2.5$ and $8.1 \mathrm{~Hz}, 1 \mathrm{H}, \mathrm{ArH}), 7.20-7.24(\mathrm{~m}, 1 \mathrm{H}, \mathrm{ArH}) .{ }^{13} \mathrm{C}$ NMR $(125 \mathrm{MHz}$, $\left.\mathrm{CDCl}_{3}\right) \delta 22.5,22.7,23.9,44.0,63.2,107.2,107.3,110.6,134.2,134.3,149.5,149.6,162.2$, 162.4. MS (EI) m/z (\%) 222 (3), 165 (100). HRMS calculated for $\left(\mathrm{C}_{12} \mathrm{H}_{15} \mathrm{~N}_{2} \mathrm{OF}\right) 222.116748$, found 222.116748. HPLC, OD-H, Hept $/{ }^{i} \mathrm{PrOH}=80: 20,220 \mathrm{~nm}, \mathrm{t}_{\text {minor }}=14.9 \mathrm{~min}, \mathrm{t}_{\text {major }}=19.5$ min. 
<smiles>COc1ccc2c(c1)C(=O)N[C@@H](CC(C)C)N2</smiles>

(R)-2-Isobutyl-6-methoxy-2,3-dihydroquinazolin-4(1H)-one 3o was prepared by the same method as that for 3a from 2-amino-5-methoxybenzamide 1i (16.6 mg, $0.1 \mathrm{mmol}$ ) and 3methylbutanal (10 $\mu \mathrm{L}, 0.1 \mathrm{mmol})$ as white solid (21.0 mg, 90\%). ${ }^{1} \mathrm{H} \mathrm{NMR} \mathrm{(500} \mathrm{MHz,} \mathrm{CDCl}_{3}$ ) $\delta 0.98\left(\mathrm{~d}, J=6.5 \mathrm{~Hz}, 3 \mathrm{H}, \mathrm{CH}_{3}\right), 0.99\left(\mathrm{~d}, J=6.5 \mathrm{~Hz}, 3 \mathrm{H}, \mathrm{CH}_{3}\right), 1.62-1.69\left(\mathrm{~m}, 2 \mathrm{H}, \mathrm{CH}_{2}\right), 1.74-$ $1.85\left(\mathrm{~m}, 1 \mathrm{H}, \mathrm{Me}_{2} \mathrm{CH}\right), 3.80\left(\mathrm{~s}, 3 \mathrm{H}, \mathrm{OCH}_{3}\right), 4.86(\mathrm{t}, J=6.2 \mathrm{~Hz}, 1 \mathrm{H}, \mathrm{NCH}), 5.93(\mathrm{~s}, 1 \mathrm{H}, \mathrm{NH})$, 6.48 (d, $J=8.7 \mathrm{~Hz}, 1 \mathrm{H}, \mathrm{ArH}), 6.96$ (dd, $J=2.9$ and $8.7 \mathrm{~Hz}, 1 \mathrm{H}, \mathrm{ArH}), 7.42$ (d, $J=2.9 \mathrm{~Hz}, 1 \mathrm{H}$, $\mathrm{ArH}) .{ }^{13} \mathrm{C}$ NMR (125 MHz, $\mathrm{CDCl}_{3}$ ) $\delta$ 22.3, 22.6, 24.0, 44.2, 55.8, 55.9, 63.9, 110.5, 117.3, 122.5, 141.4, 153.6, 165.5. MS (EI) m/z (\%) 234 (8), 177 (100). HRMS calculated for $\left(\mathrm{C}_{13} \mathrm{H}_{18} \mathrm{~N}_{2} \mathrm{O}_{2}\right)$ 234.136827, found 234.136606. HPLC, OD-H, Hept $/{ }^{i} \mathrm{PrOH}=80: 20,220 \mathrm{~nm}$, $\mathrm{t}_{\text {minor }}=17.8 \mathrm{~min}, \mathrm{t}_{\text {major }}=22.0 \mathrm{~min}$.<smiles>CC(C)C[C@@H]1NC(=O)c2cc(OC(F)(F)F)ccc2N1</smiles>

(R)-2-Isobutyl-6-(trifluoromethoxy)-2,3-dihydroquinazolin-4(1H)-one 3p was prepared by the same method as that for 3a from 2-amino-5-(trifluoromethoxy)benzamide 1j (22.0 mg, 0.1 mmol) and 3-methylbutanal (10 $\mathrm{L}, 0.1 \mathrm{mmol}$ ) as white solid (27.6 mg, 96\%). ${ }^{1} \mathrm{H}$ NMR (500 $\left.\mathrm{MHz} \mathrm{CDCl}_{3}\right) \delta 0.99\left(\mathrm{~d}, J=6.5 \mathrm{~Hz}, 3 \mathrm{H}, \mathrm{CH}_{3}\right), 1.00$ (d, $\left.J=6.5 \mathrm{~Hz}, 3 \mathrm{H}, \mathrm{CH}_{3}\right), 1.65-1.73(\mathrm{~m}$, $2 \mathrm{H}, \mathrm{CH}_{2}$ ), 1.76-1.84 (m, 1H, $\left.\mathrm{Me}_{2} \mathrm{CH}\right), 4.94(\mathrm{t}, J=6.2 \mathrm{~Hz}, 1 \mathrm{H}, \mathrm{NCH}), 6.19(\mathrm{~s}, 1 \mathrm{H}, \mathrm{NH}), 6.68$ (d, $J=8.7 \mathrm{~Hz}, 1 \mathrm{H}, \mathrm{ArH}), 7.17$ (d, $J=8.7 \mathrm{~Hz}, 1 \mathrm{H}, \mathrm{ArH}), 7.76$ (s, $1 \mathrm{H}, \mathrm{ArH}) .{ }^{13} \mathrm{C}$ NMR $(125$ $\left.\mathrm{MHz} \mathrm{CDCl}_{3}\right) \delta 22.5,22.6,23.9,44.3,63.7,116.0,116.8,121.4,127.3,141.9,145.9,164.0$. MS (EI) m/z (\%) 288 (4), 231 (100). HRMS calculated for $\left(\mathrm{C}_{13} \mathrm{H}_{15} \mathrm{~N}_{2} \mathrm{O}_{2} \mathrm{~F}_{3}\right)$ 288.108563, found 288.108281. HPLC, OD-H, Hept ${ }^{i} \mathrm{PrOH}=80: 20,220 \mathrm{~nm}, \mathrm{t}_{\text {minor }}=8.4 \mathrm{~min}, \mathrm{t}_{\text {major }}=9.7$ $\min$.<smiles>Cc1ccc2c(c1C)N[C@H](CC(C)C)NC2=O</smiles>

(R)-2-Isobutyl-7,8-dimethyl-2,3-dihydroquinazolin-4(1H)-one $\mathbf{3 q}$ was prepared by the same method as that for 3a from 2-amino-3,4-dimethylbenzamide 1k $(22.0 \mathrm{mg}, 0.1 \mathrm{mmol})$ and 3methylbutanal (10 $\mu \mathrm{L}, 0.1 \mathrm{mmol})$ as white solid (19.7 mg, 85\%). ${ }^{1} \mathrm{H}$ NMR (300 $\mathrm{MHz}, \mathrm{CDCl}_{3}$ ) $\delta 0.98\left(\mathrm{~d}, J=6.5 \mathrm{~Hz}, 6 \mathrm{H}, \mathrm{CH}_{3}\right), 1.67-1.72\left(\mathrm{~m}, 2 \mathrm{H}, \mathrm{CH}_{2}\right), 1.74-1.90\left(\mathrm{~m}, 1 \mathrm{H}, \mathrm{Me}_{2} \mathrm{CH}\right), 2.06$ (s, $\left.3 \mathrm{H}, \mathrm{ArCH}_{3}\right), 2.89$ (s, 3H, $\left.\mathrm{ArCH}_{3}\right), 4.05$ (b, 1H, NH), 4.84-4.89 (m, 1H, NCH), 6.72 (d, $J=8.0$ $\mathrm{Hz}, 1 \mathrm{H}, \mathrm{ArH}), 6.70$ (b, 1H, NH), 7.69 (d, $J=8.0 \mathrm{~Hz}, 1 \mathrm{H}, \mathrm{ArH}) .{ }^{13} \mathrm{C}$ NMR $\left(75 \mathrm{MHz}, \mathrm{CDCl}_{3}\right) \delta$ 12.2, 20.9, 22.6, 24.1, 44.4, 63.4, 114.6, 120.8, 121.4, 125.6, 142.1, 145.5, 166.1. MS (EI) $\mathrm{m} / \mathrm{z}$ (\%) 232 (4), 175 (100). HRMS(ESI) calculated for $\left(\mathrm{C}_{14} \mathrm{H}_{20} \mathrm{~N}_{2} \mathrm{ONa}\right) 255.146779$, found 255.146748. HPLC, IA, Hept ${ }^{i} \mathrm{PrOH}=80: 20,220 \mathrm{~nm}, \mathrm{t}_{\text {minor }}=12.9 \mathrm{~min}, \mathrm{t}_{\text {major }}=13.7 \mathrm{~min}$.<smiles>Cc1cc(Cl)cc2c1N[C@@H](CC(C)C)NC2=O</smiles>

(R)-5-Chloro-3-methyl-2-isobutyl-2,3-dihydroquinazolin-4(1H)-one $3 \mathbf{r}$ was prepared by the same method as that for 3a from 2-amino-3-methy-5-cholorbenzamide 11 (15 mg, $0.1 \mathrm{mmol}$ ) 
and butyraldehyde (10 $\mu \mathrm{L}, 0.1 \mathrm{mmol})$ as white solid (19.5 mg, 84\%). ${ }^{1} \mathrm{H}$ NMR (500 MHz, $\left.\mathrm{CDCl}_{3}\right) \delta 0.91$ (d, $\left.J=6.5 \mathrm{~Hz}, 6 \mathrm{H}, \mathrm{CH}_{3}\right), 1.58-1.68\left(\mathrm{~m}, 2 \mathrm{H}, \mathrm{CH}_{2}\right), 1.71-1.77(\mathrm{~m}, 1 \mathrm{H}, \mathrm{MeCH})$, $2.06\left(\mathrm{~s}, 3 \mathrm{H}, \mathrm{CH}_{3}\right), 4.81(\mathrm{t}, J=6.2 \mathrm{~Hz}, 1 \mathrm{H}, \mathrm{NCH}), 7.07-7.1(\mathrm{~m}, 2 \mathrm{H}), 7.66(\mathrm{~s}, 1 \mathrm{H}, \mathrm{ArH}) .{ }^{13} \mathrm{C}$ NMR (125 MHz, $\left.\mathrm{CDCl}_{3}\right) \delta 16.4,22.5,44.3,63.3,117.0,123.4,124.0,124.6,125.8,134.2$, 114.0, 164.6. MS (EI) m/z (\%) 252 (6), 197 (32), 195 (100). HRMS(ESI) calculated for $\left(\mathrm{C}_{13} \mathrm{H}_{17} \mathrm{ClN}_{2} \mathrm{ONa}\right)$ 275.092158, found 275.092166. HPLC, OD-H, Hept ${ }^{i} \mathrm{PrOH}=80: 20,254$ $\mathrm{nm}, \mathrm{t}_{\text {minor }}=15.4$ min, $\mathrm{t}_{\text {major }}=16.8 \mathrm{~min}$.<smiles>CC(C)C[C@@H]1Nc2ccccc2S(=O)(=O)N1</smiles>

(R)-2H-1,2,4-Benzothiadiazine,3,4-dihydro-3-isobutyl-,1,1-dioxide 8 was prepared by the same method as that for 3a from 2-aminobenzenesulfonamide 7 (17.2 mg, $0.1 \mathrm{mmol}$ ) and 3methylbutanal ( $10 \mu \mathrm{L}, 0.1 \mathrm{mmol})$, but the compound racemized quickly at room temperature during workup and purifiecation (silica gel, Hex/EA = 3:1, v/v, $19.3 \mathrm{mg}, 80 \%$ ). ${ }^{1} \mathrm{H}$ NMR $\left(500 \mathrm{MHz}, \mathrm{CDCl}_{3}\right) \delta 1.01\left(\mathrm{~d}, J=6.6 \mathrm{~Hz}, 3 \mathrm{H}, \mathrm{CH}_{3}\right), 1.02(\mathrm{~d}, J=6.6 \mathrm{~Hz}, 3 \mathrm{H}, \mathrm{ArH}), 1.56-1.62$ (m, $1 \mathrm{H}$, one $\mathrm{H}$ of $\left.\mathrm{CH}_{2}\right), 1.71-1.78\left(\mathrm{~m}, 1 \mathrm{H}\right.$, one $\mathrm{H}$ of $\left.\mathrm{CH}_{2}\right), 1.87-1.97(\mathrm{~m}, 1 \mathrm{H}, \mathrm{MeCH}), 4.25(\mathrm{~d}$, $J=13 \mathrm{~Hz}, 1 \mathrm{H}, \mathrm{NH}), 4.97-5.03(\mathrm{~m}, 1 \mathrm{H}, \mathrm{NCH}), 6.67$ (d, $J=8.0 \mathrm{~Hz}, 1 \mathrm{H}, \mathrm{NH}), 6.85$ (t, $J=7.8$ $\mathrm{Hz}, 1 \mathrm{H}, \mathrm{ArH}), 7.29(\mathrm{t}, J=8.4 \mathrm{~Hz}, 1 \mathrm{H}, \mathrm{ArH}), 7.64$ (d, $J=8.0 \mathrm{~Hz}, 1 \mathrm{H}, \mathrm{ArH}) .{ }^{13} \mathrm{C}$ NMR $(125$ $\left.\mathrm{MHz}, \mathrm{CDCl}_{3}\right) \delta 22.3$, 22.6, 24.1, 43.8, 64.8, 116.2, 119.0, 122.8, 124.9, 133.3, 142.4. MS (EI) m/z (\%) 240 (14), 183 (100), 156 (10), 108 (14), 92 (40). HRMS calculated for $\left(\mathrm{C}_{11} \mathrm{H}_{16} \mathrm{~N}_{2} \mathrm{O}_{2} \mathrm{~S}\right)$ 240.093322, found 240.093254. HPLC, OD-H, Hept/iPrOH = 80:20, $254 \mathrm{~nm}$, $\mathrm{t}_{\text {major }}=14.7 \mathrm{~min}$, $\mathrm{t}_{\text {minor }}=31.1 \mathrm{~min}$.

Preparation of catalyst (S)-Ad-TRIP 4e

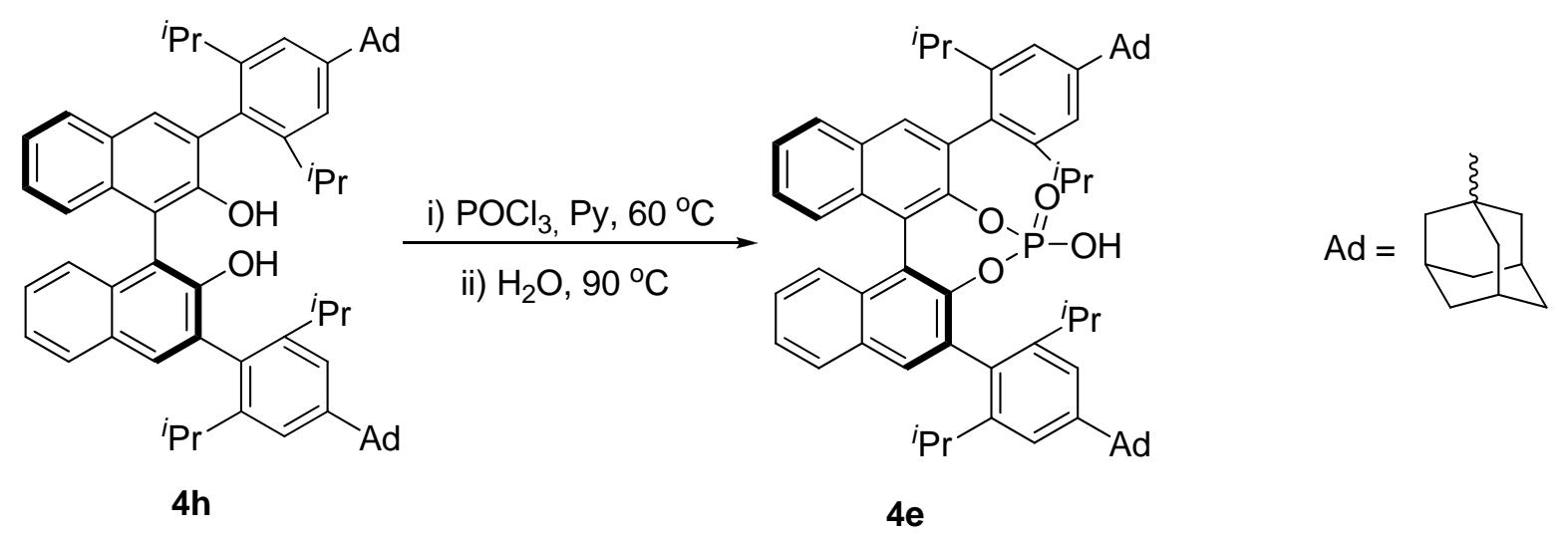

To a solution of diol $\mathbf{4 h}{ }^{1}$ (370 $\mathrm{mg}, 0.423 \mathrm{mmol}$ ) in anhydrous pyridine ( $3 \mathrm{~mL}$ ) was added phosphoryl trichloride $(100 \mu \mathrm{L}, 1.1 \mathrm{mmol})$ under argon at $60{ }^{\circ} \mathrm{C}$. After stirring at $60{ }^{\circ} \mathrm{C}$ overnight, the solution was cooled to $0{ }^{\circ} \mathrm{C}$ and added water $(0.5 \mathrm{~mL}, 27.8 \mathrm{mmol})$ and heated to $90{ }^{\circ} \mathrm{C}$ for 6 hours. Then the mixture was cooled to room temperature,added $20 \mathrm{~mL}$ water and extracted with DCM three times. The combined organic phase was washed with $1 \mathrm{~N} \mathrm{HCl}$, dried over $\mathrm{MgSO}_{4}$ and concentrated to give yellow oil that was purified by chromatography on silica gel with Hex/EA (3:1, v/v) as mobile phase to give $\mathbf{4 e}$ as white foam (366 mg, 92\%). $[\alpha]_{\mathrm{d}}{ }^{20}=+38.4$ (c 1.0, EA). ${ }^{1} \mathrm{H}$ NMR (500 MHz, DMSO) $\delta 0.88$ (d, $\left.J=6.8 \mathrm{~Hz}, 6 \mathrm{H}, \mathrm{CH}_{3}\right), 1.09$ (d, $\left.J=6.8 \mathrm{~Hz}, 6 \mathrm{H}, \mathrm{CH}_{3}\right), 1.16-1.18\left(\mathrm{~m}, 12 \mathrm{H}, \mathrm{CH}_{3}\right), 1.77$ (s, $\left.12 \mathrm{H}, \mathrm{CH}_{2}\right), 1.97\left(\mathrm{~s}, 12 \mathrm{H}, \mathrm{CH}_{2}\right)$, 2.09 (s, 6H, CH in admantyl), 2.57-2.64 (m, 2H, Me $2 \mathrm{CH}$ ), 2.96-3.01 (m, 2H, $\mathrm{Me}_{2} \mathrm{CH}$ ), 7.05 (d, $J=8.6 \mathrm{~Hz}, 2 \mathrm{H}, \mathrm{ArH}), 7.14$ (s, 2H, ArH), 7.23 (s, 2H, ArH), 7.27 (t, $J=7.8 \mathrm{~Hz}, 2 \mathrm{H}, \mathrm{ArH})$, 
7.39 (t, $J=7.3 \mathrm{~Hz}, 2 \mathrm{H}, \mathrm{ArH}), 7.79$ (s, 2H, ArH), 7.97 (d, $J=7.97,2 \mathrm{H}, \mathrm{ArH}) .{ }^{13} \mathrm{C}$ NMR $(125$ MHz, DMSO) 23.2, 23.4, 24.6, 26.3, 28.4, 30.1, 30.7, 35.8, 36.3, 42.7, 117.6, 118.9, 122.2, 124.0, 125.5, 128.1, 129.5, 130.8, 132.1, 133.1, 133.5, 145.7, 147.0, 149.1, 149.3. ${ }^{31} \mathrm{P}$ NMR (202 MHz, DMSO) $\delta$ 3.36. MS (EI) m/z (\%) 936 (23), 893 (17), 135 (100). HRMS(ESI, M) calculated for $\left(\mathrm{C}_{64} \mathrm{H}_{72} \mathrm{O}_{4} \mathrm{P}\right)$ 935.517371, found 935.517722 .

Synthesis of $(R)$-Thiabutazide

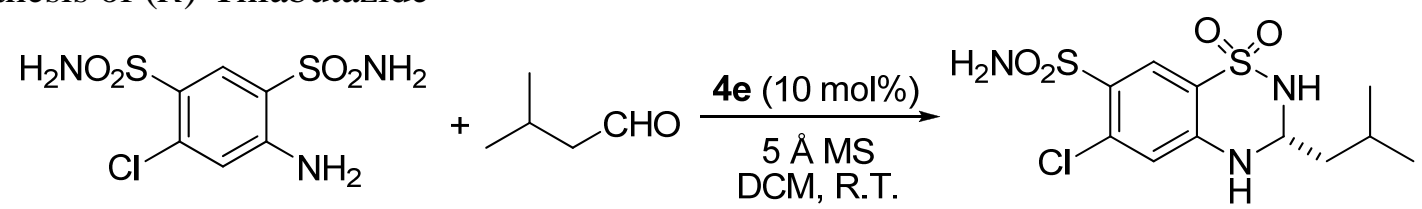

To a reaction vial charged with 4-amino-6-chlorobenzene-1,3-disulfonamide (28.4 mg, 0.1 mmol), (S)-Ad-TRIP 4e (9.6 mg, $0.01 \mathrm{mmol})$ and $30 \mathrm{mg} 5 \AA$ MS was added DCM (1 mL) and 3-methylbutanal (10 $\mu \mathrm{L}, 0.1 \mathrm{mmol})$, followed by stirring at room temperature for 168 hours. Then the reaction mixture was concentrated and purified by silica gel chromatography with Hex/EA (4:6, v/v) as eluent to give the (R)-Thiabutazide as white solid (28.6 mg, 81\%). ${ }^{1} \mathrm{H}$ NMR (400 MHz, CD $\left.{ }_{3} \mathrm{OD}\right) \delta 0.85\left(\mathrm{t}, J=6.3 \mathrm{~Hz}, 6 \mathrm{H}, \mathrm{CH}_{3}\right), 1.48-1.54\left(\mathrm{~m}, 1 \mathrm{H}\right.$, one $\mathrm{H}$ of $\left.\mathrm{CH}_{2}\right)$, 1.60-1.67 (m, $1 \mathrm{H}$, one $\mathrm{H}$ of $\left.\mathrm{CH}_{2}\right), 1.73-1.83(\mathrm{~m}, 1 \mathrm{H}, \mathrm{MeCH}), 4.25(\mathrm{~d}, J=13 \mathrm{~Hz}, 1 \mathrm{H}, \mathrm{NH})$, 4.67-4.73 (m, 1H, NCH), 6.90 (s, 1H, ArH), 7.40 (s, 1H, NH N $^{2}, 7.70$ (d, $J=12.0 \mathrm{~Hz}, 1 \mathrm{H}, \mathrm{NH}$ ), 7.75 (s, 1H, NH), 7.90 (s, 1H, ArH). ${ }^{13} \mathrm{C}$ NMR (100 MHz, CD $\left.{ }_{3} \mathrm{OD}\right) \delta 22.1,23.0,23.8,42.0$, 64.7, 64.8, 117.3, 117.4, 118.6, 125.9, 128.5, 134.6, 146.9. MS (EI) m/z (\%) 353 (The spectrum is very complicated). HRMS calculated for $\left(\mathrm{C}_{11} \mathrm{H}_{15} \mathrm{~N}_{3} \mathrm{O}_{4} \mathrm{~S}_{2} \mathrm{Cl}\right) 352.019804$, found 352.020124. $[\alpha]_{\mathrm{d}}{ }^{20}=-86.5^{\circ}$ (c 0.75, EA, er 95.5:4.5). HPLC, OD-H, Hept ${ }^{i} \mathrm{PrOH}=80: 20$, $220 \mathrm{~nm}, \mathrm{t}_{\text {major }}=20.5 \mathrm{~min}, \mathrm{t}_{\text {minor }}=36.6 \mathrm{~min}$.

The preparation of $(R)$-Bendroflumethiazide

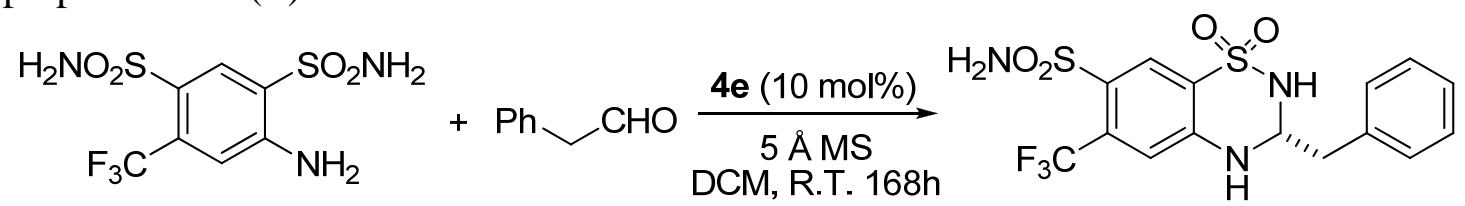

The compound (16.8 mg, 80\%) was prepared from 4-amino-6-(trifluoromethyl)benzene-1,3disulfonamide (16 mg, $0.05 \mathrm{mmol}$ ) and phenyl acetaldehyde ( $9 \mu \mathrm{L}, 0.05 \mathrm{mmol}$ ) with the same method as that for $(R)$-Thiabutazide using $(S)$-4e as catalyst. ${ }^{1} \mathrm{H}$ NMR $\left(500 \mathrm{MHz}, \mathrm{CD}_{3} \mathrm{OD}\right) \delta$ 2.98-3.10 (m, 2H, $\mathrm{CH}_{2} \mathrm{Ph}$ ), 5.02 (dd, $J=8.8$ and $\left.5.0 \mathrm{~Hz}, 1 \mathrm{H}, \mathrm{NCH}\right), 7.16-7.27$ (m, 6H, ArH), 1.64-1.70 (m, 2H, CH $)$, 8.25 (1H, s, ArH). ${ }^{13} \mathrm{C}$ NMR (125 MHz, $\left.\mathrm{CD}_{3} \mathrm{OD}\right) \delta 40.9,68.7,117.0$, 117.1, 123.4, 128.2, 128.7, 129.6, 130.0, 130.6, 132.0, 132.3, 136.5, 147.4. MS (EI) m/z (\%) 421 (8), 330 (100), 319 (75), 302 (20), 255 (15), 158 (13), 118 (18), 91 (26). HPLC, OD-H, $\mathrm{Hept} /{ }^{i} \mathrm{PrOH}=80: 20,220 \mathrm{~nm}, \mathrm{t}_{\text {major }}=39.3 \mathrm{~min}, \mathrm{t}_{\text {minor }}=49.7 \mathrm{~min}$.

The preparation of $(R)$-Penflutizide

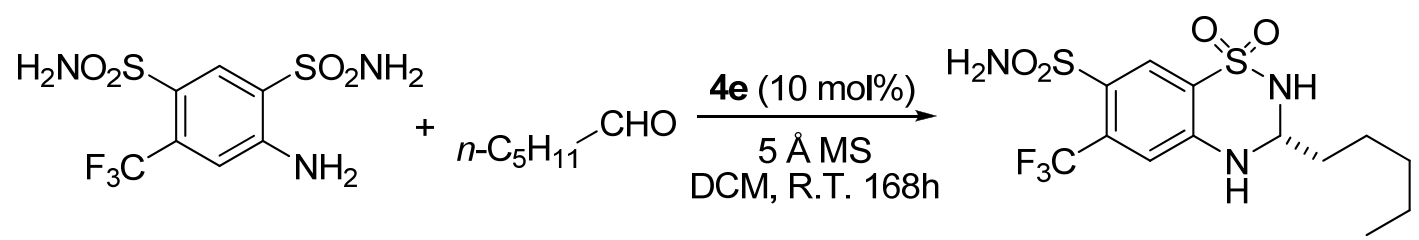

The compound (14.9 mg, 74\%) was prepared from 4-amino-6-(trifluoromethyl)benzene-1,3disulfonamide (16 mg, $0.05 \mathrm{mmol}$ ) and n-Hexaldehyde (8 $\mu \mathrm{L}, 0.05 \mathrm{mmol}$ ) with the same method as that for $(R)$-Thiabutazide using $(S)-4 \mathbf{e}$ as catalyst. ${ }^{1} \mathrm{H}$ NMR (500 MHz, $\left.\mathrm{CD}_{3} \mathrm{OD}\right) \delta$ 
1.15-1.27 (m, 2H, $\mathrm{CH}_{2}$ ), 1.61-1.67 (m, 2H, $\left.\mathrm{CH}_{2}\right), 1.68-1.75$ (m, 2H, $\left.\mathrm{CH}_{2}\right), 1.77-1.83(\mathrm{~m}, 1 \mathrm{H}$, one $\mathrm{H}$ of $\left.\mathrm{CH}_{2}\right), 1.90-1.96\left(\mathrm{~m}, 3 \mathrm{H}, \mathrm{CH}_{3}\right), 2.06-2.18\left(\mathrm{~m}, 1 \mathrm{H}\right.$, one $\mathrm{H}$ of $\left.\mathrm{CH}_{2}\right), 4.87(\mathrm{~m}, 1 \mathrm{H}$, the peaks overlap with solvent peak), 6.97 (s, $1 \mathrm{H}, \mathrm{ArH}), 8.18$ (s, $1 \mathrm{H}, \mathrm{ArH}) .{ }^{13} \mathrm{C}$ NMR $(125 \mathrm{MHz}$, $\left.\mathrm{CD}_{3} \mathrm{OD}\right) \delta 25.8,25.9,33.3,37.0,41.0,67.1,118.5,120.3,127.7,129.6,136.6,148.4$. HPLC, OD-H, Hept $/{ }^{i} \mathrm{PrOH}=80: 20,220 \mathrm{~nm}, \mathrm{t}_{\text {major }}=22.9 \mathrm{~min}, \mathrm{t}_{\mathrm{minor}}=36.6 \mathrm{~min}$.

The preparation of $(R)$-Cyclopenthiazide

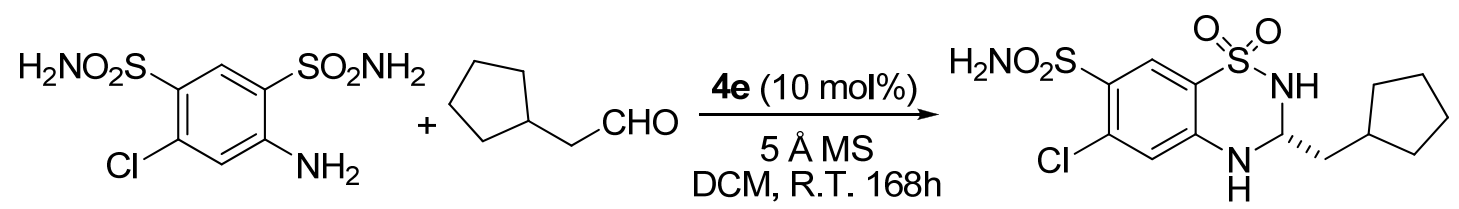

The compound (13.7 mg, 74\%) was prepared from 4-amino-6-chlorobenzene-1,3disulfonamide (14 mg, $0.05 \mathrm{mmol}$ ) and 2-cyclopentylacetaldehyde (7 $\mu \mathrm{L}, 0.05 \mathrm{mmol})$ with the same method as that for $(R)$-Thiabutazide using $(S)-4 \mathbf{e}$ as catalyst. ${ }^{1} \mathrm{H}$ NMR $(500 \mathrm{MHz}$, $\left.\mathrm{CD}_{3} \mathrm{OD}\right) \delta$ 0.96-0.98 (m, 3H, $\mathrm{CH}_{2}$ and $\left.\mathrm{CH}\right), 1.41-1.44\left(\mathrm{~m}, 4 \mathrm{H}, \mathrm{CH}_{2}\right), 1.51-1.66\left(\mathrm{~m}, 2 \mathrm{H}, \mathrm{CH}_{2}\right)$, 1.82-1.92 (m, $\left.2 \mathrm{H}, \mathrm{CH}_{2}\right), 4.90$ (m, $1 \mathrm{H}$, the peaks overlap with solvent peak), 7.30 (s, $\left.1 \mathrm{H}, \mathrm{ArH}\right)$, 8.37 (s, 1H, ArH). ${ }^{13} \mathrm{C}$ NMR (125 MHz, CD 3 OD) $\delta 14.3,23.5,25.2,32.5,34.7,67.6,116.9$, 117.0, 122.8, 123.2, 125.0, 128.8, 129.7, 132.0, 132.2, 147.5. MS (EI) m/z (\%) 379 (8), 296 (100), 287 (14), 285 (32), 268 (21), 247 (13), 221 (20), 205 (18), 110 (17), 43 (39). HPLC, $\mathrm{OD}-\mathrm{H}, \mathrm{Hept} /{ }^{i} \mathrm{PrOH}=80: 20,220 \mathrm{~nm}, \mathrm{t}_{\mathrm{major}}=24.9 \mathrm{~min}, \mathrm{t}_{\mathrm{minor}}=43.5 \mathrm{~min}$.

The Preparation of $(S)$-Aquamox

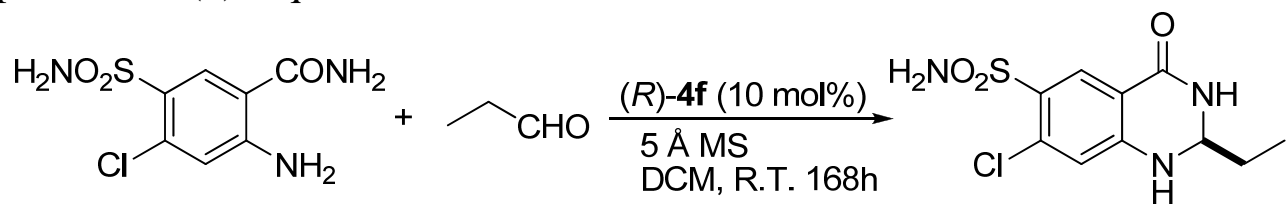

To a reaction vial charged with 2-amino-4-chloro-5-sulfamoylbenzamide (12.5 mg, 0.05 mmol), (R)-4f (3 mg, $0.005 \mathrm{mmol})$ and $5 \AA$ MS (15 mg) was added DCM (1 mL) and propionaldehyde ( $4 \mu \mathrm{L}, 0.05 \mathrm{mmol}$ ). The suspension was stirring at room temperature for 168 hours and concentrated. The residue was purified by silica gel chromatography (EA) to give Aquamox as white solid (11 mg, 78\%). ${ }^{1} \mathrm{H}$ NMR (500 MHz, $\left.\mathrm{CD}_{3} \mathrm{OD}\right) \delta 0.91(\mathrm{t}, J=7.5 \mathrm{~Hz}$, $\left.3 \mathrm{H}, \mathrm{CH}_{3}\right), 1.64-1.70\left(\mathrm{~m}, 2 \mathrm{H}, \mathrm{CH}_{2}\right), 4.75(1 \mathrm{H}, \mathrm{NCH}$, the peak overlaped with solvent signal), 6.76 (s ,1H, ArH), 8.26 (s, 1H, ArH). ${ }^{13} \mathrm{C}$ NMR (125 MHz, CD $\left.\mathrm{OD}\right) \delta$ 7.99, 30.1, 67.1, 112.6, 117.1, 130.2, 131.3, 137.9, 152.7, 165.2. MS (EI) m/z (\%) 289 (3), 260 (100), 180 (20), 145 (7). HRMS (ESI) calculated for $\left(\mathrm{C}_{10} \mathrm{H}_{12} \mathrm{~N}_{3} \mathrm{O}_{3} \mathrm{SClNa}\right) 312.018011$, found 312.018309. HPLC, OJ-H, Hept $/{ }^{i} \mathrm{PrOH}=70: 30,220 \mathrm{~nm}, \mathrm{t}_{\text {major }}=22.8 \mathrm{~min}, \mathrm{t}_{\text {minor }}=28.5 \mathrm{~min}$. 


\title{
4 NMR Spectra
}

\author{
4.1 Substrates
}



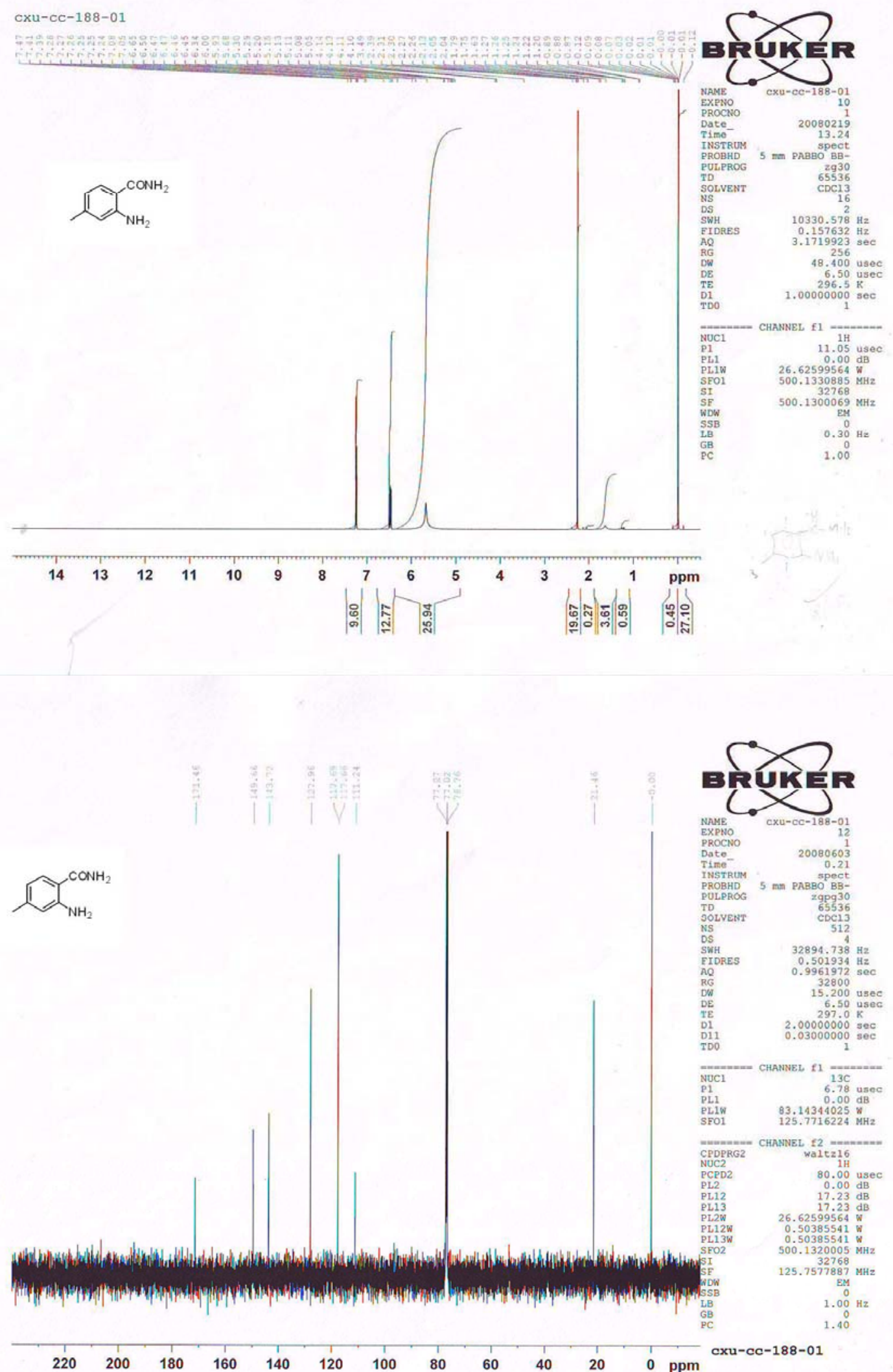


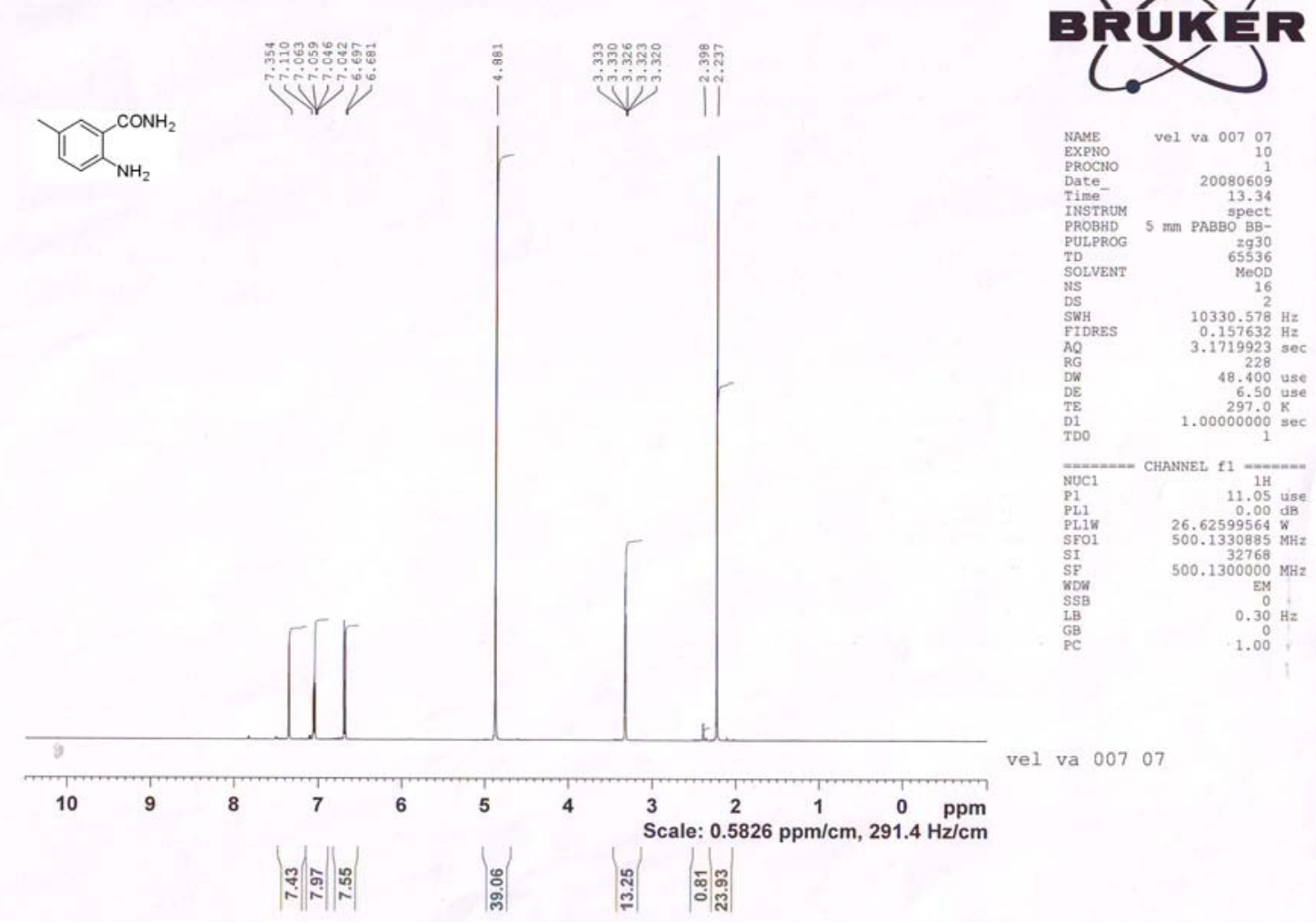

vel va 00707

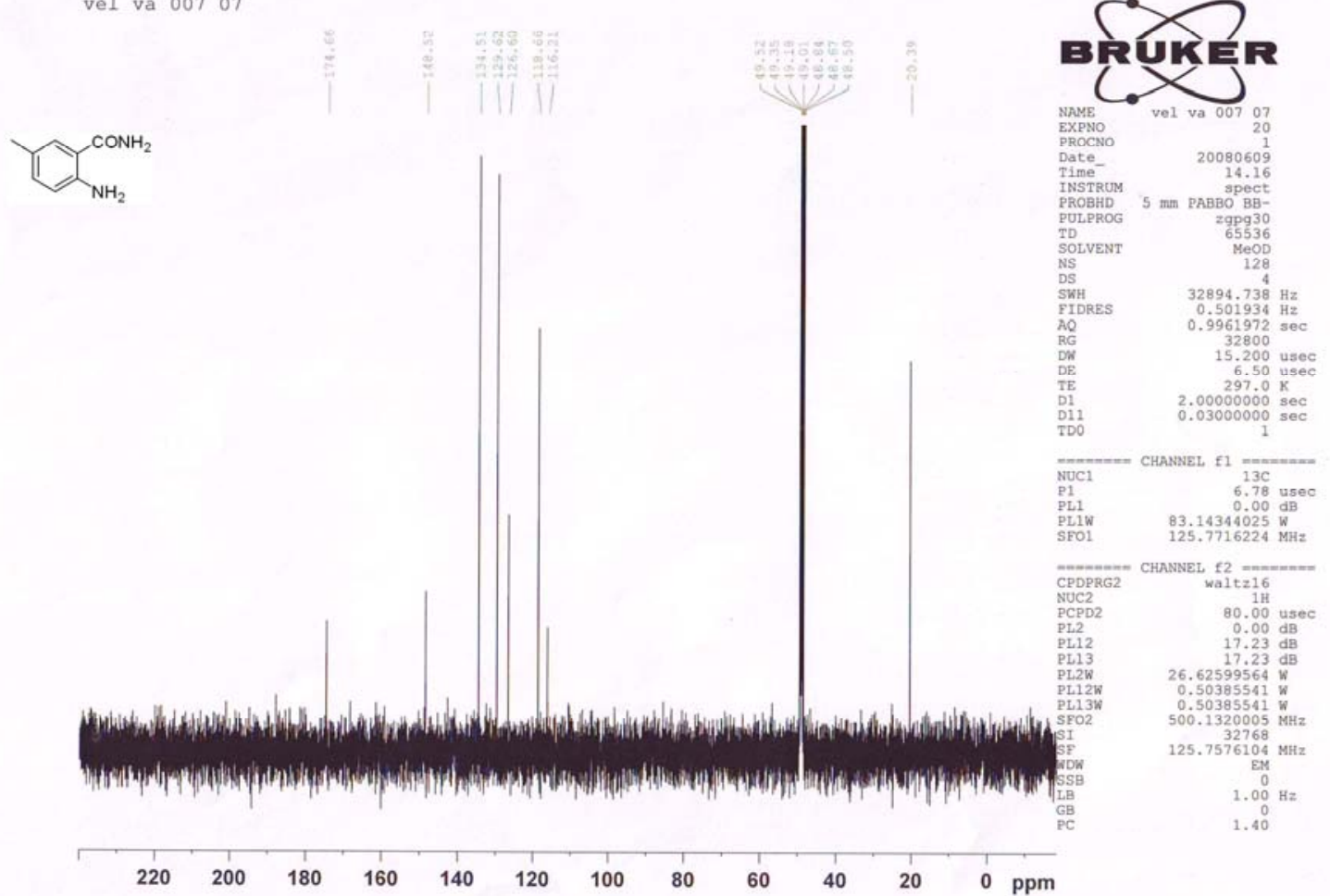



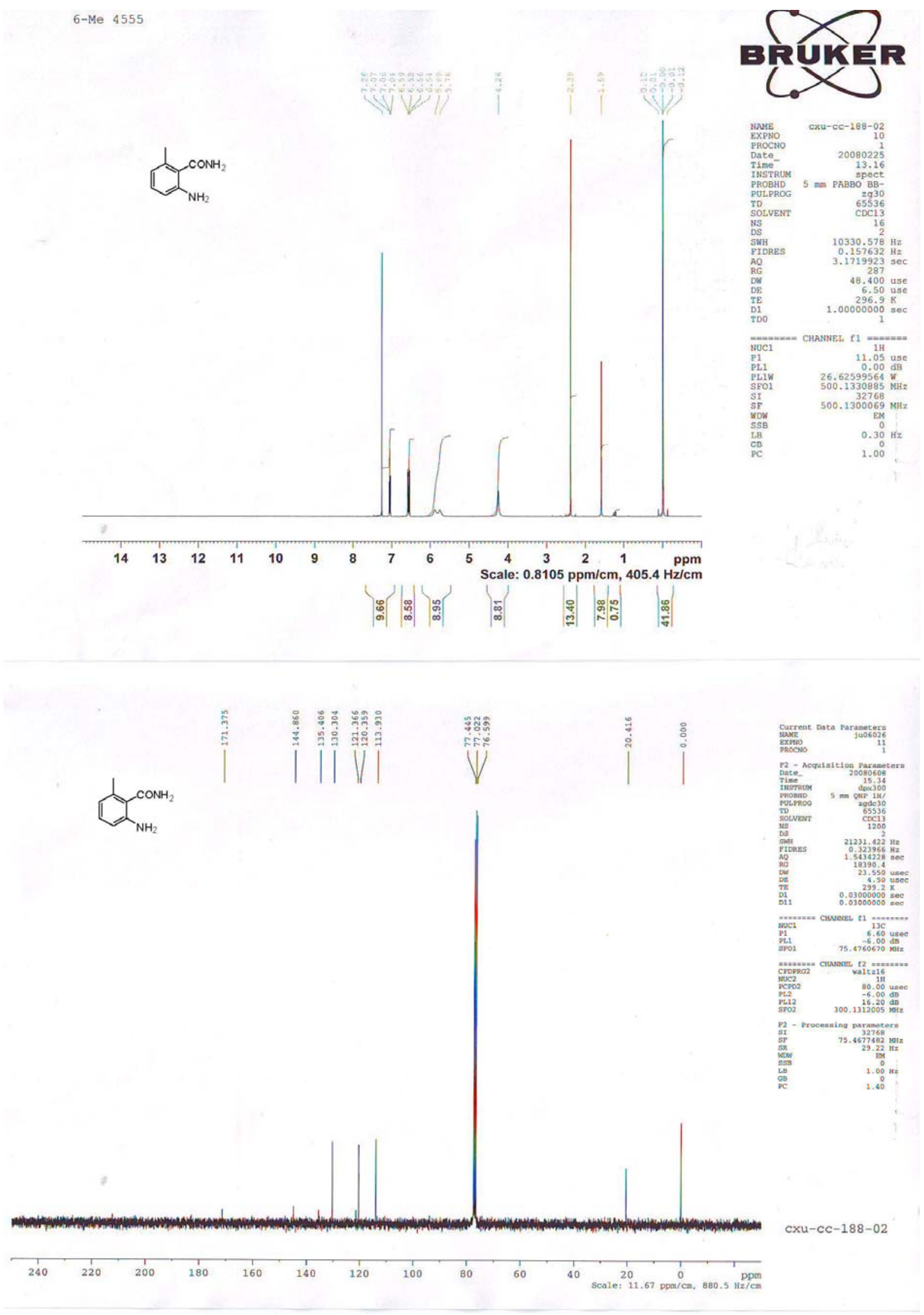

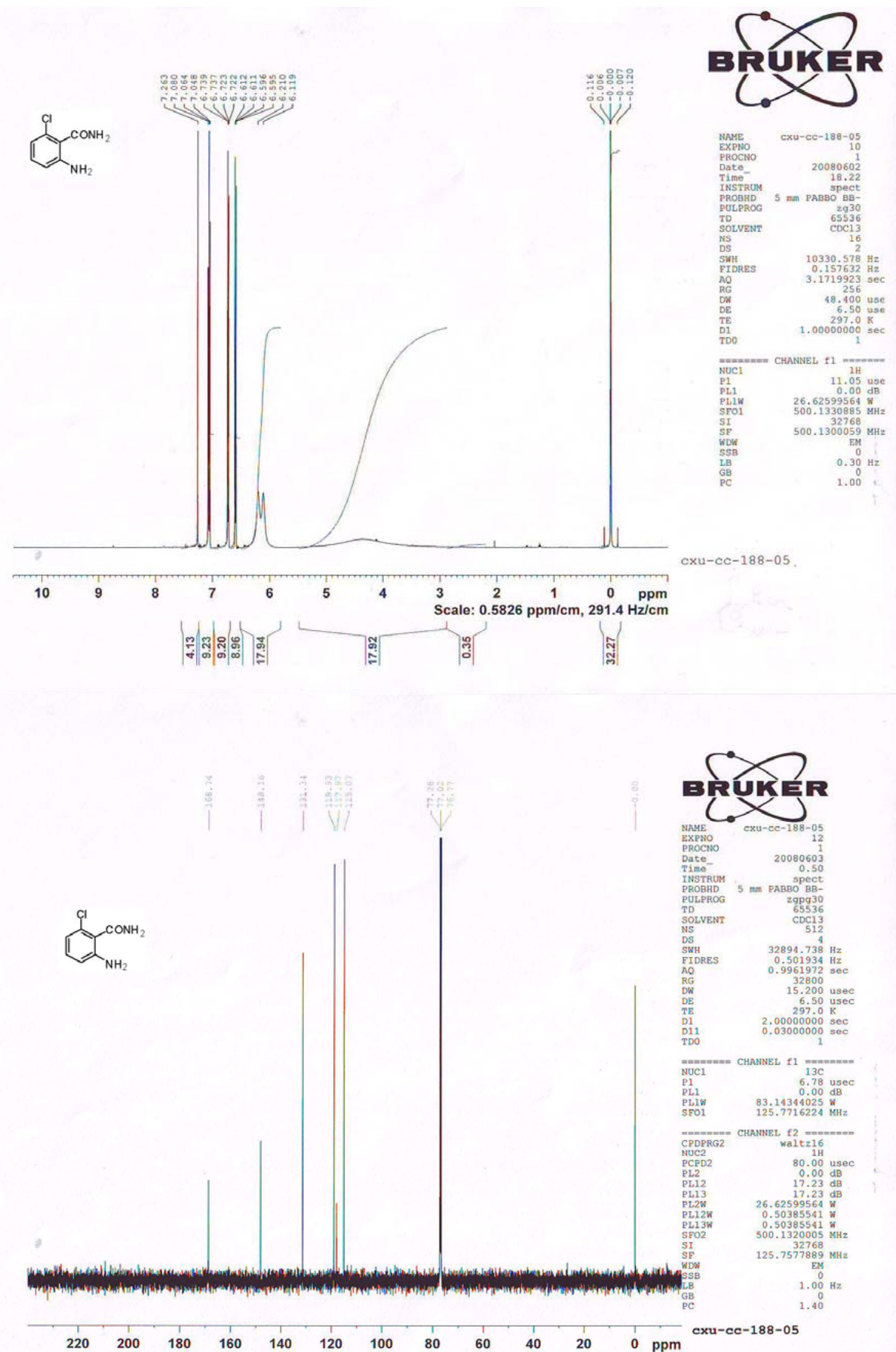


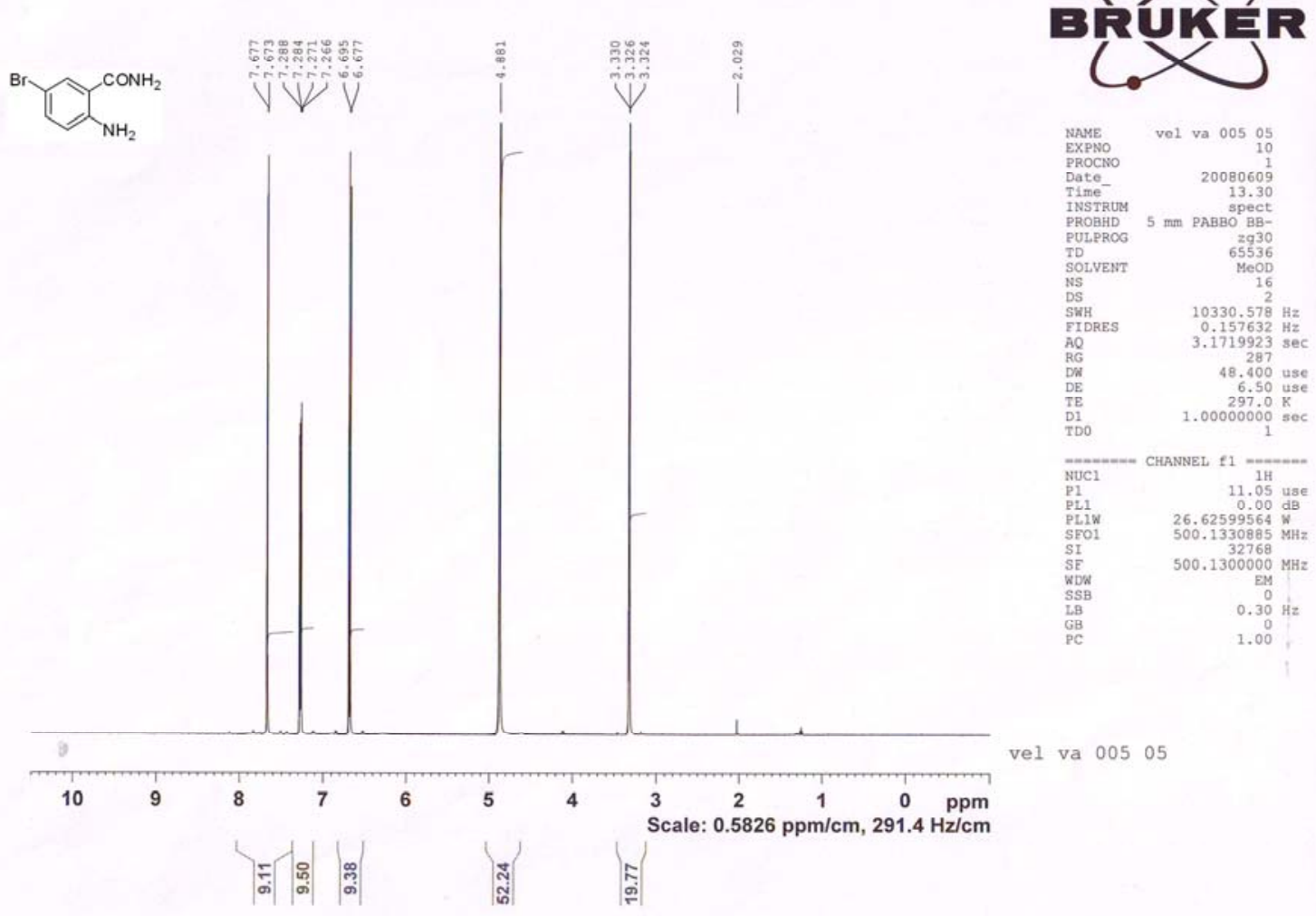

vel va $00505 \mathrm{c}$
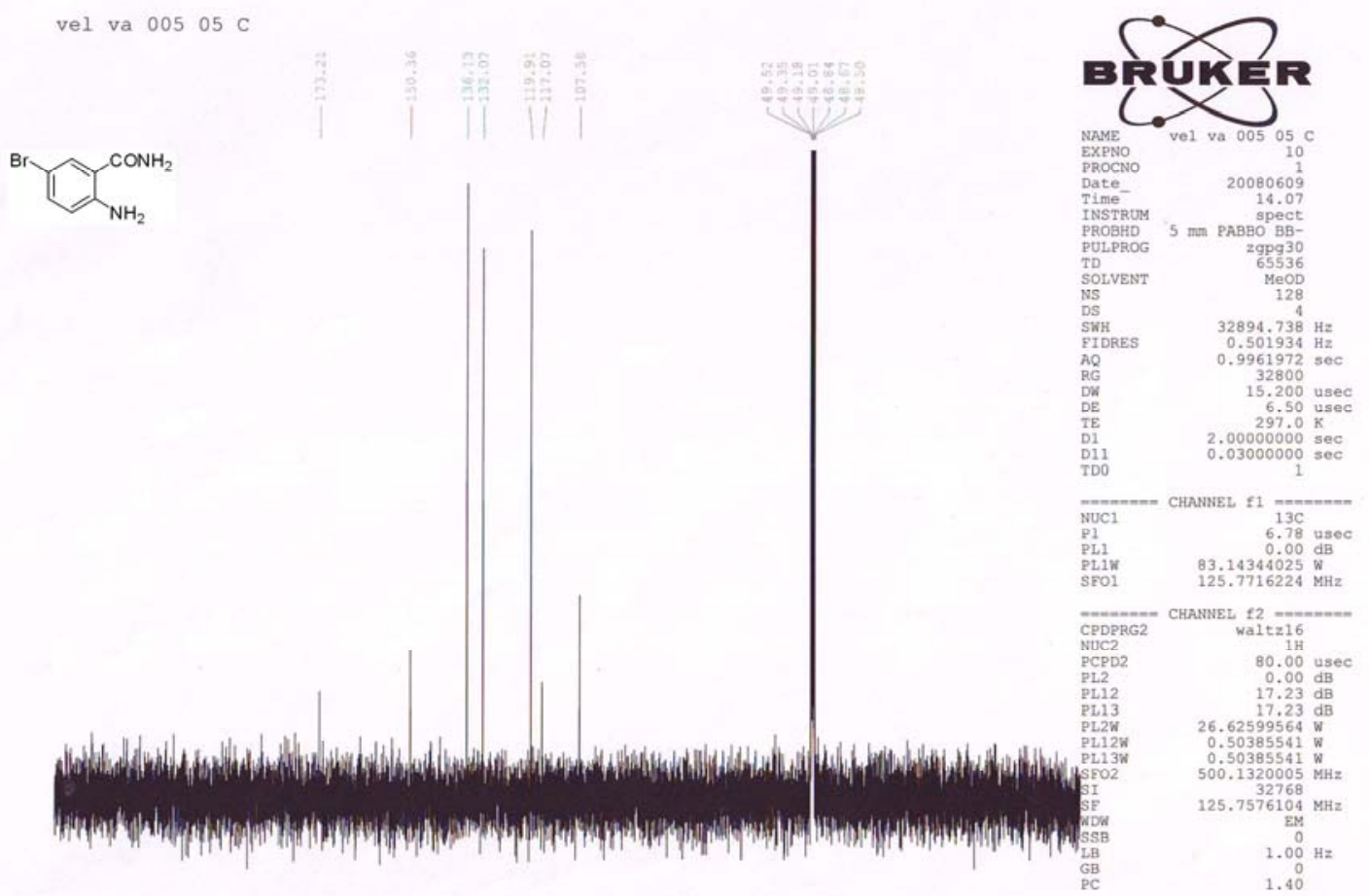

$\begin{array}{llllllllllll}220 & 200 & 180 & 160 & 140 & 120 & 100 & 80 & 60 & 40 & 20 & 0\end{array}$ 

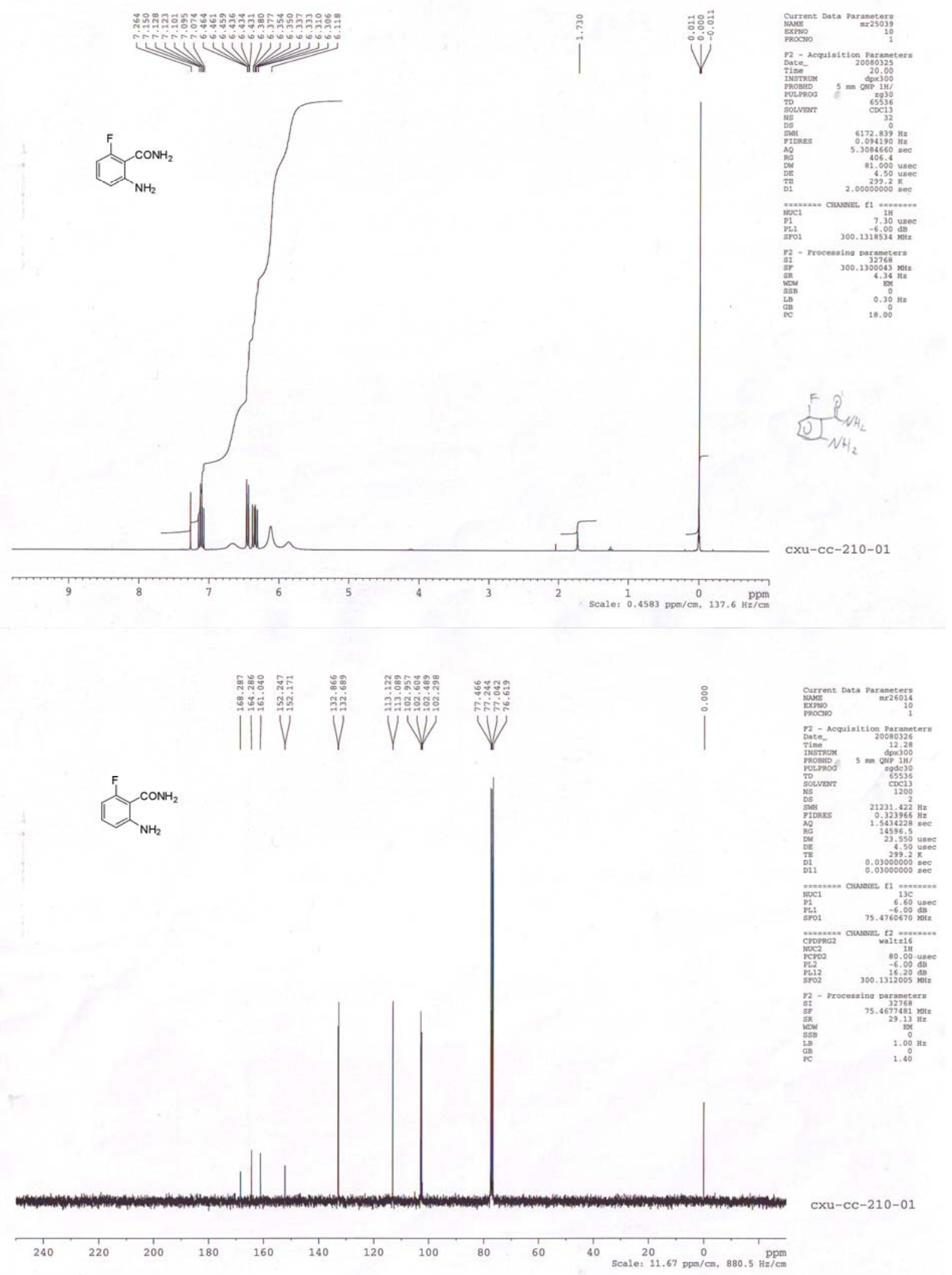

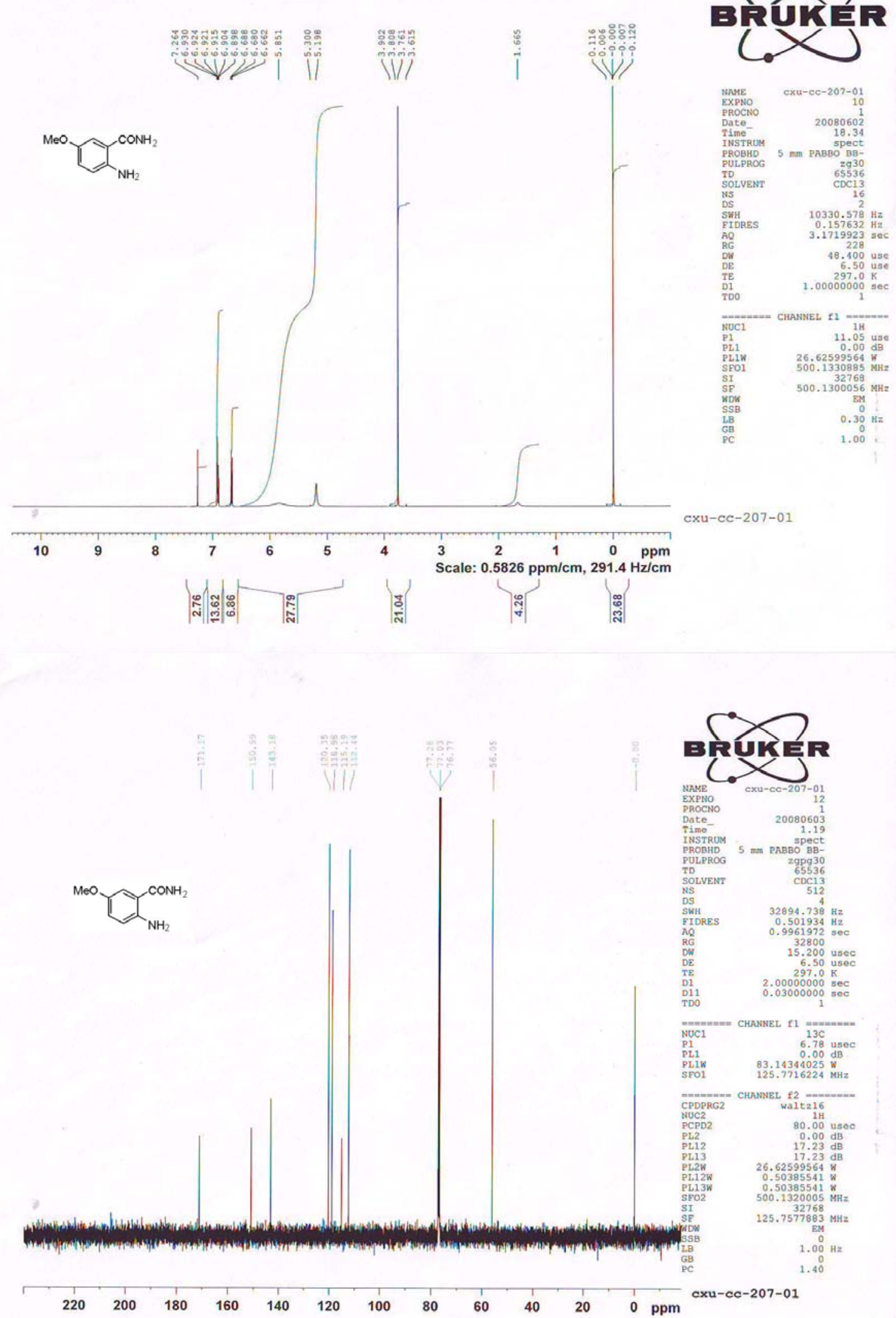


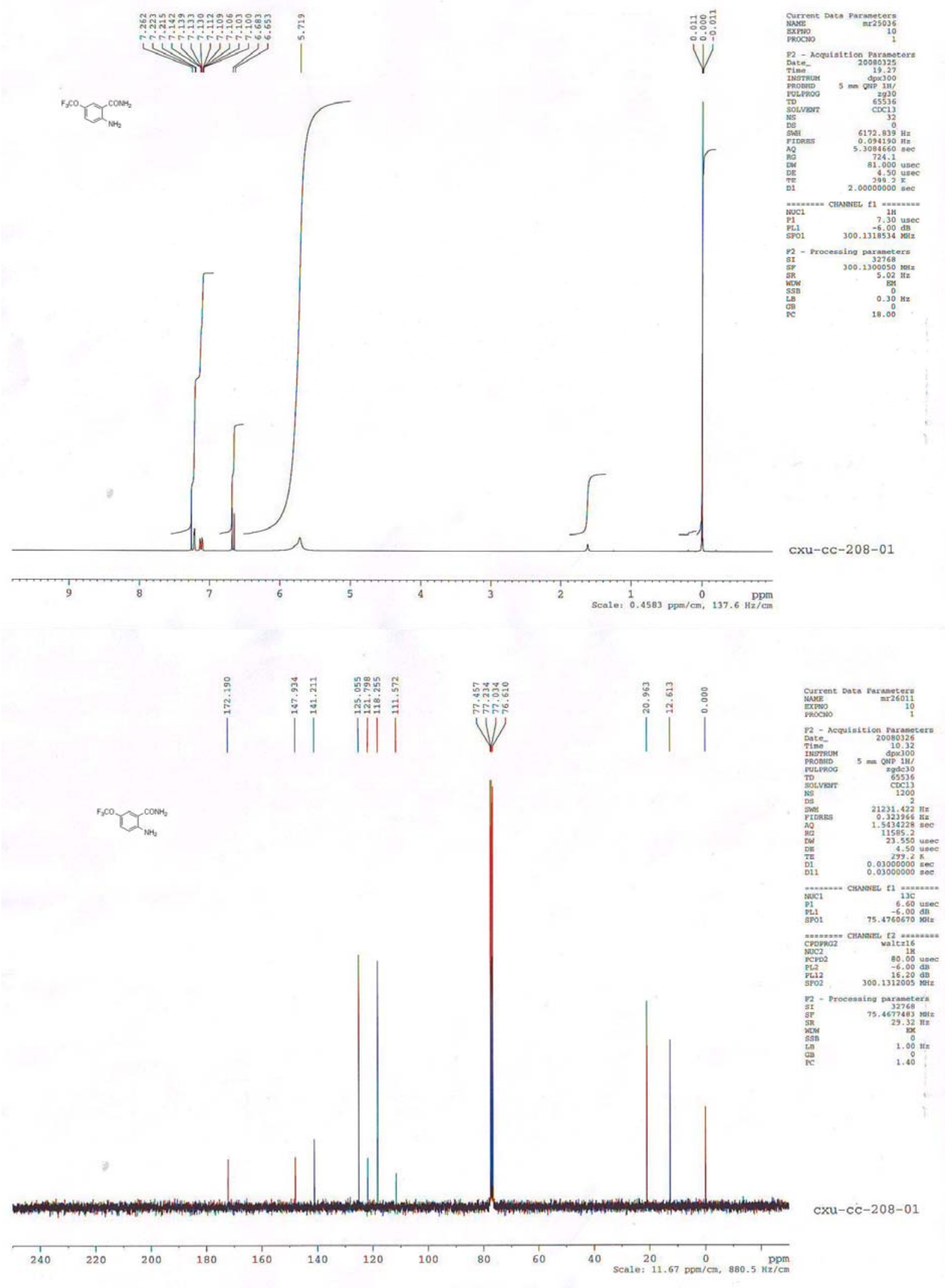



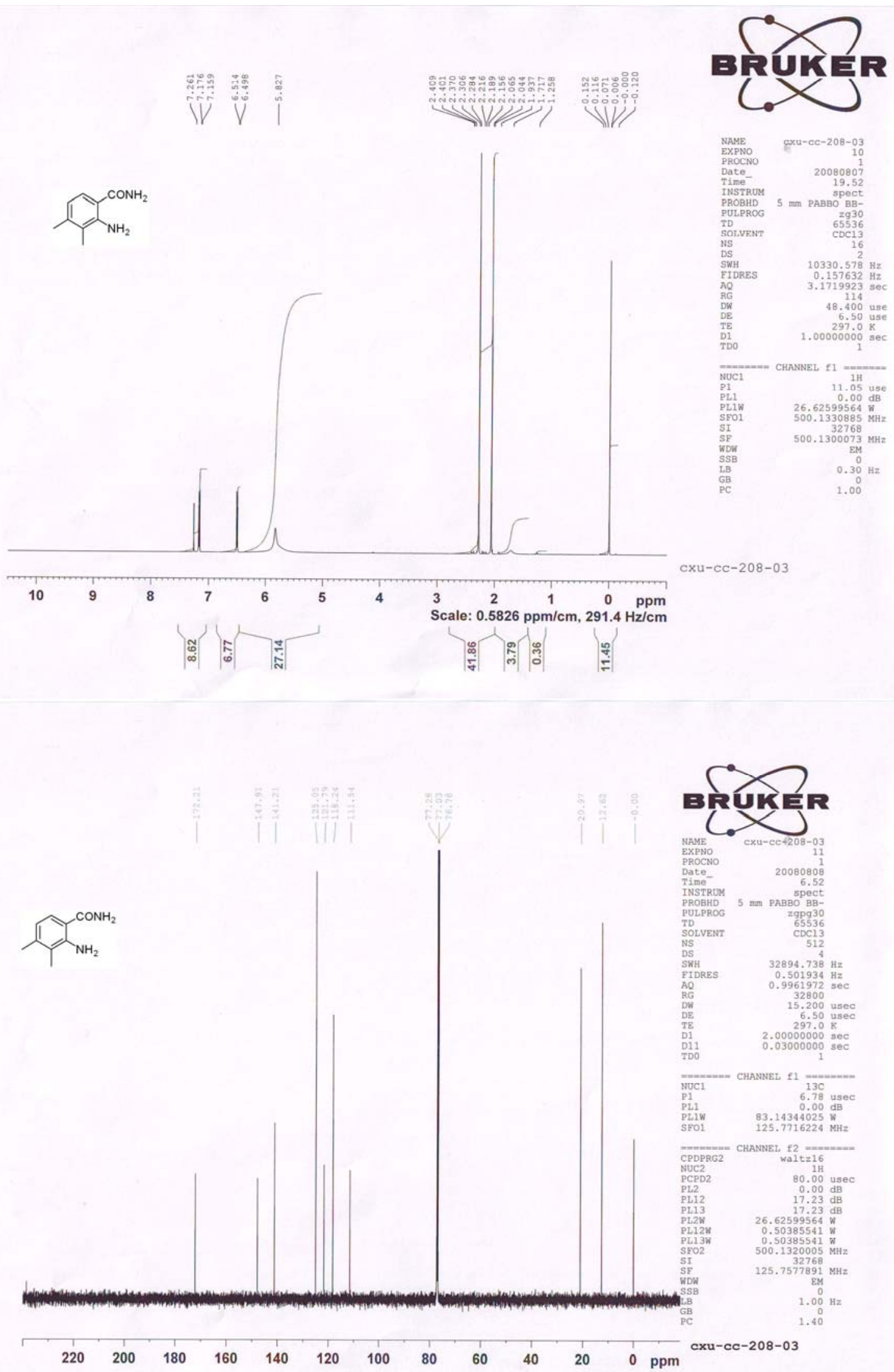

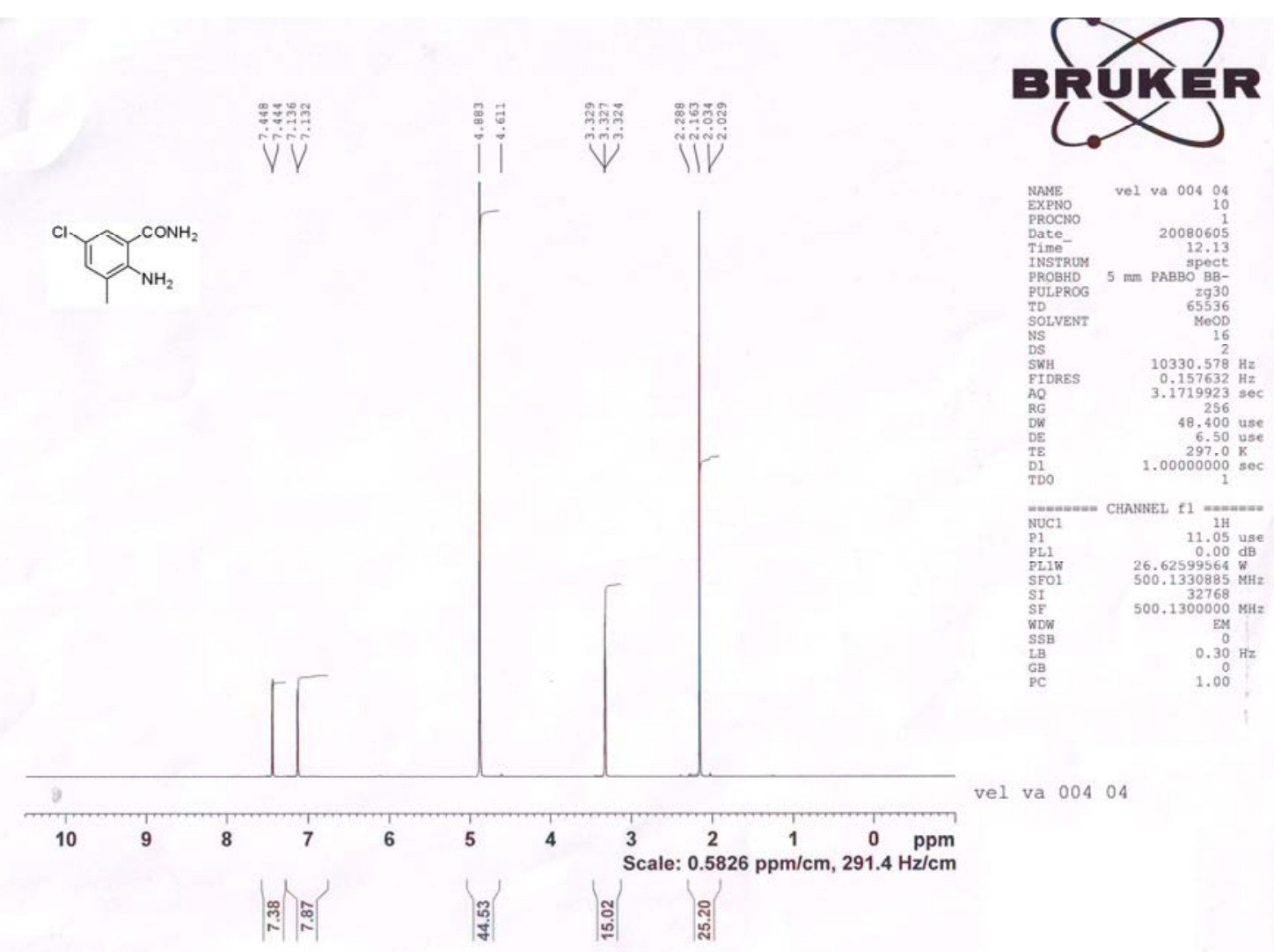

vel va $00404 \mathrm{C}$
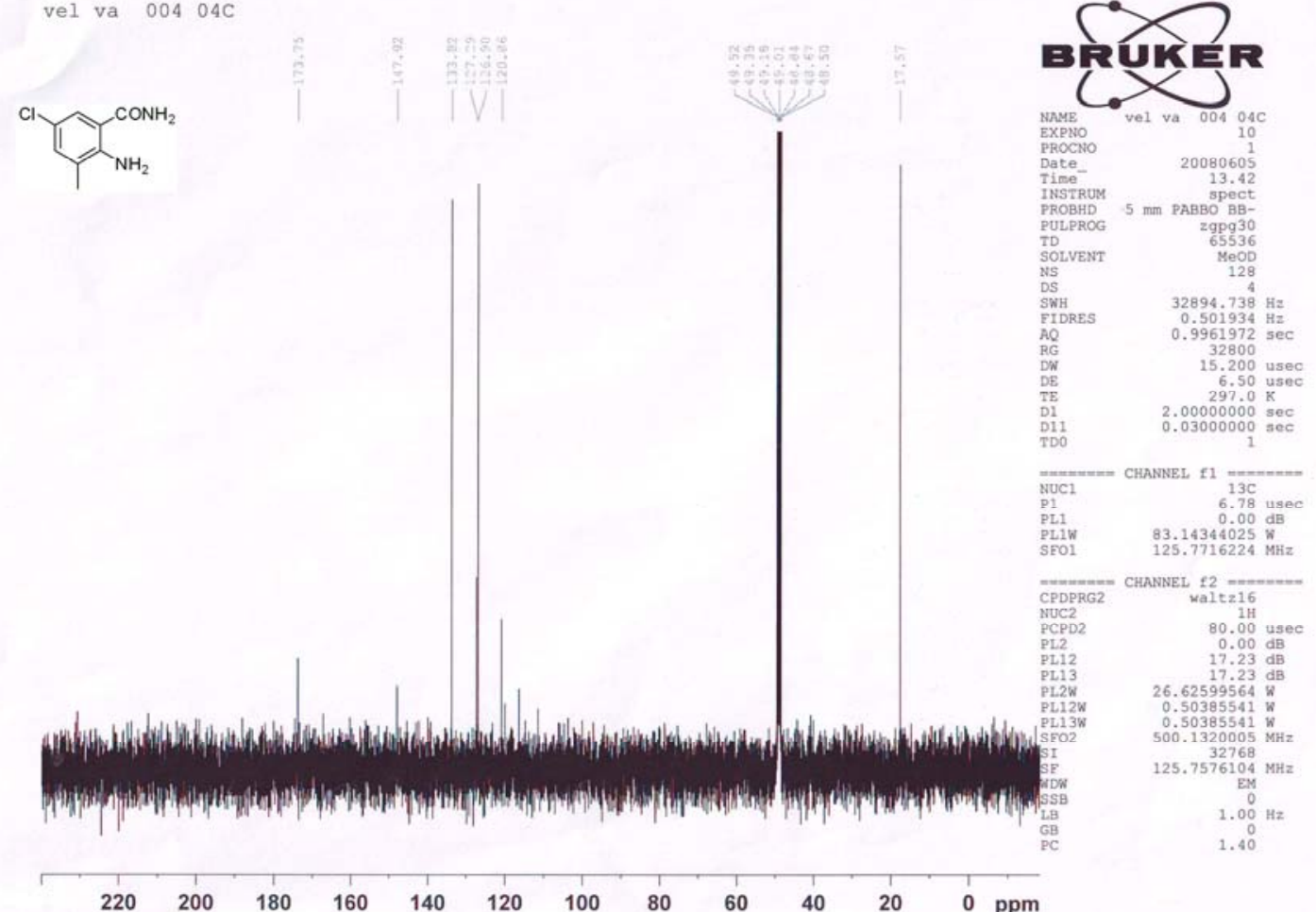


\subsection{Products}
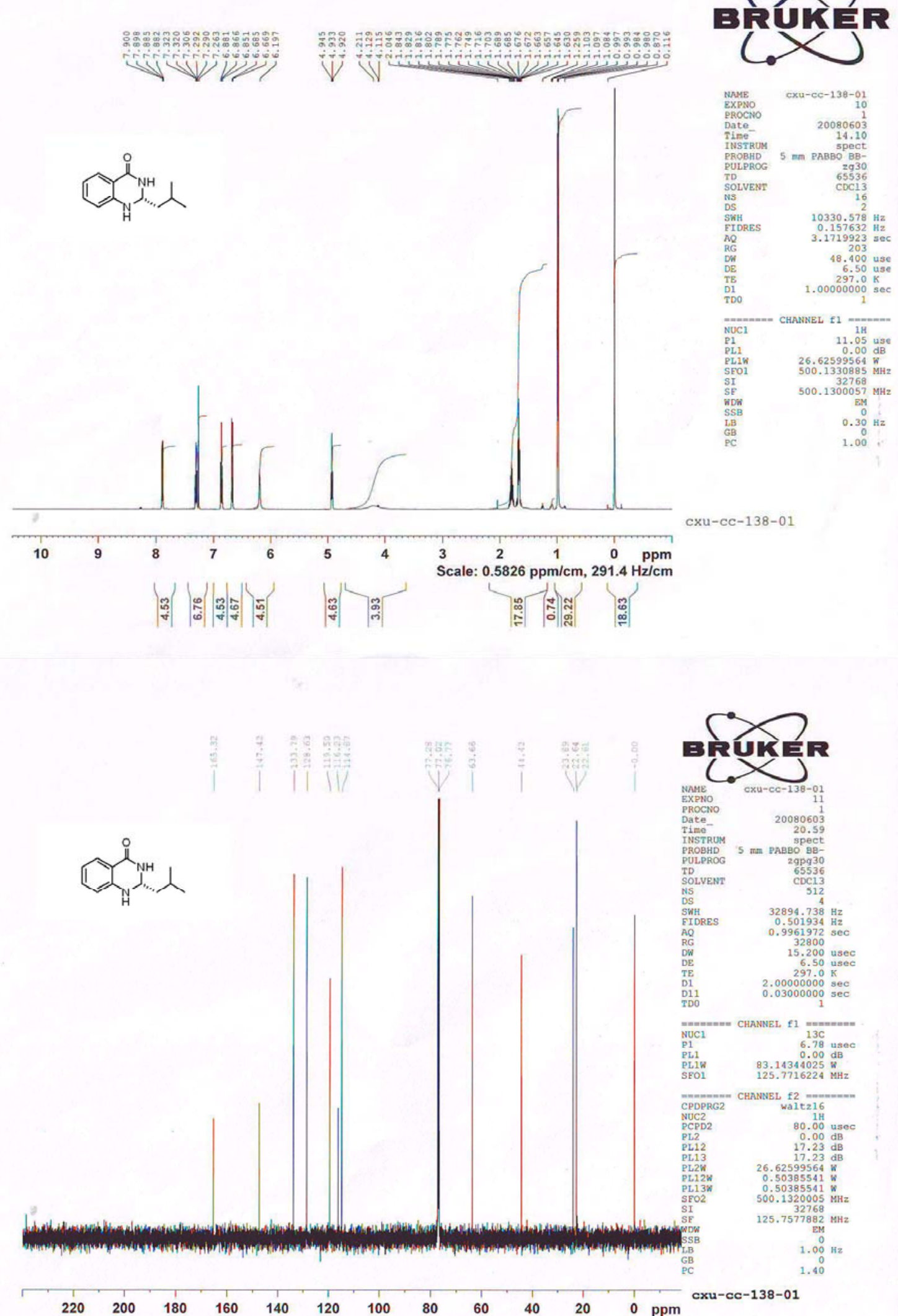

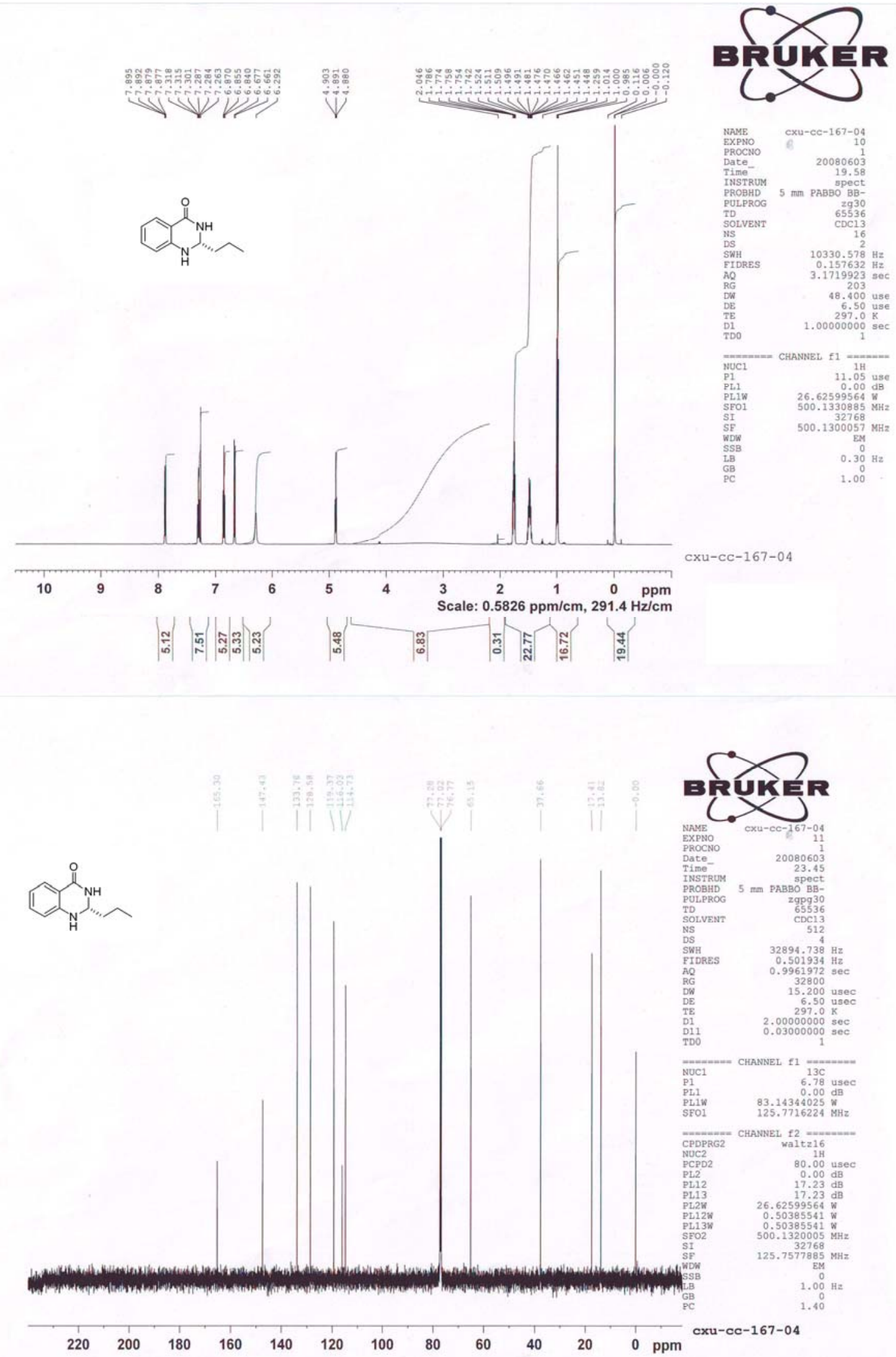
ร<smiles>CC(C)(C)CCNc1cccc2ccccc12</smiles>
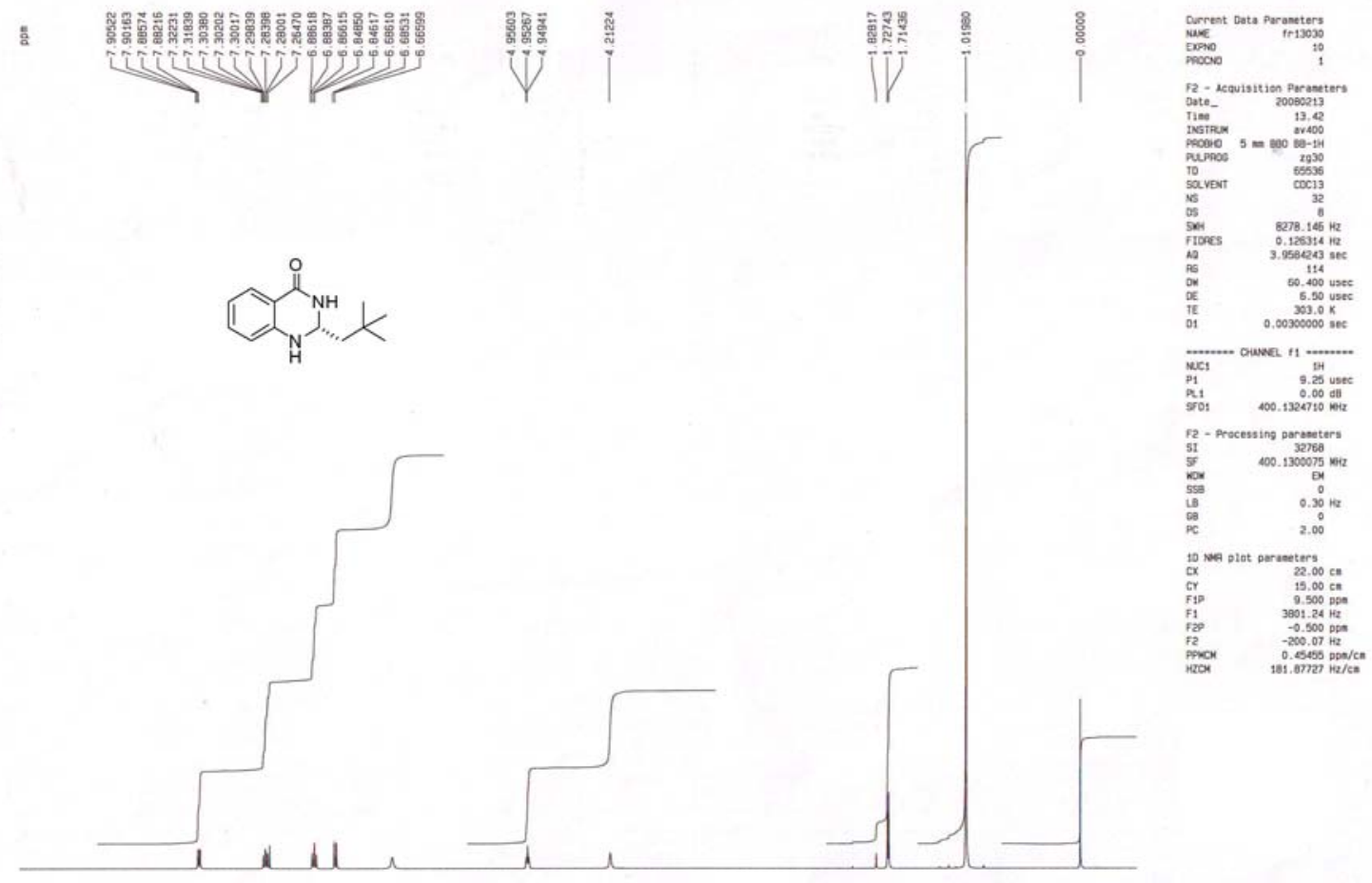

$$
\text { ppm }
$$

ฉ

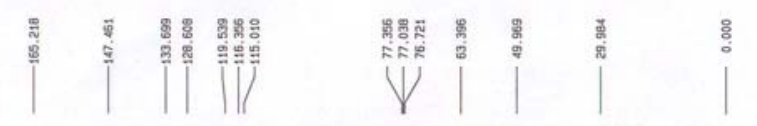

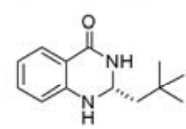
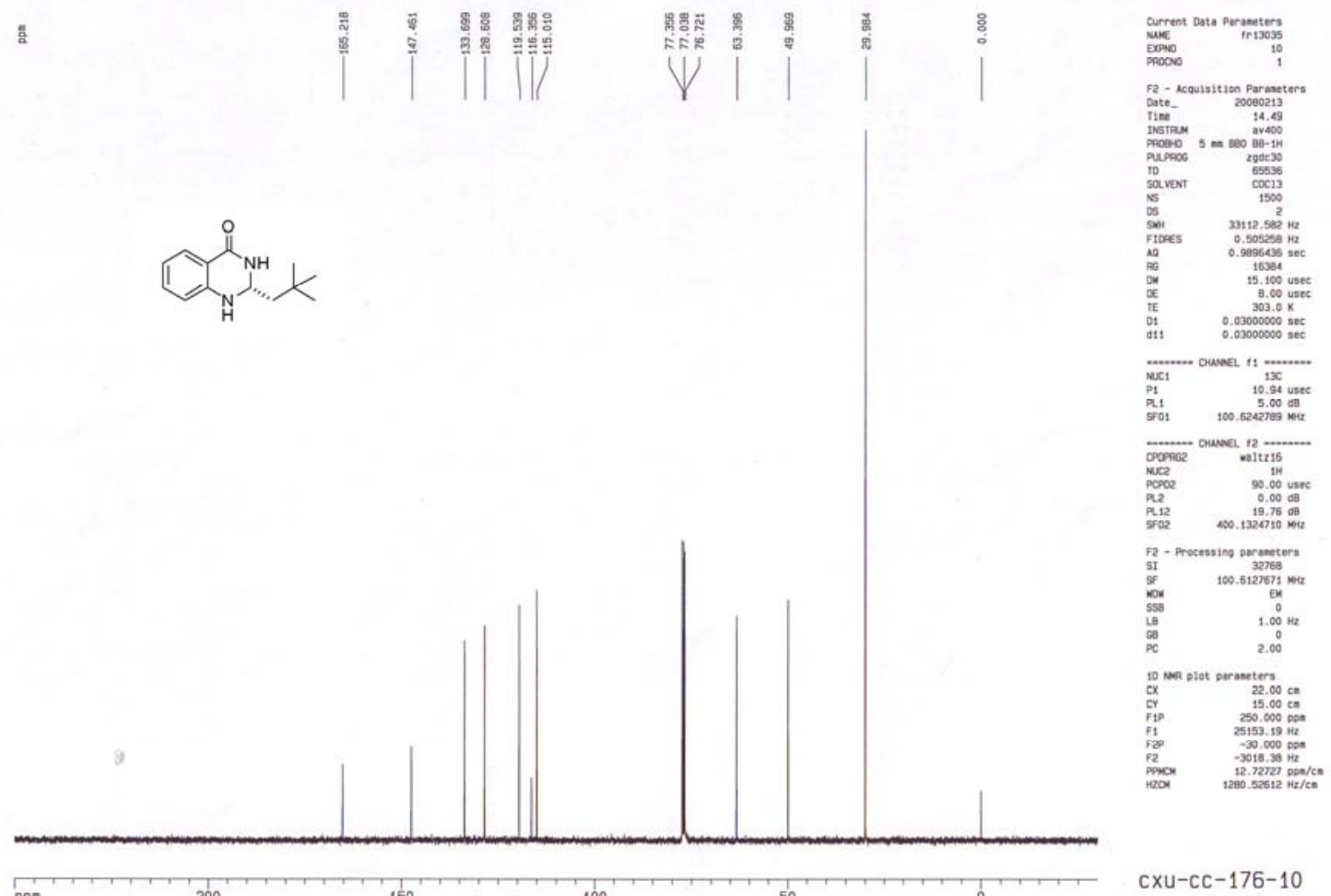

cxu-cc-176-10 

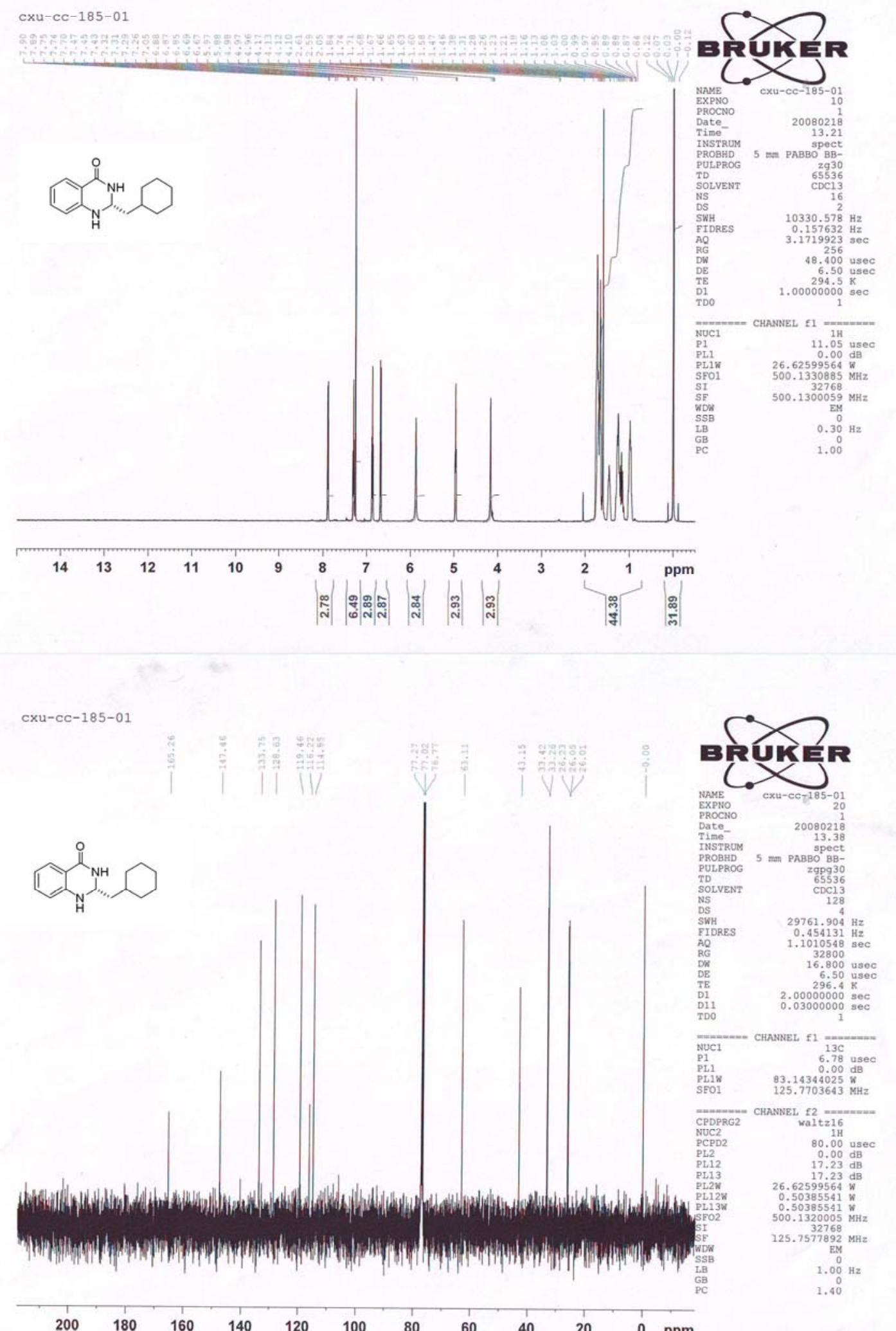

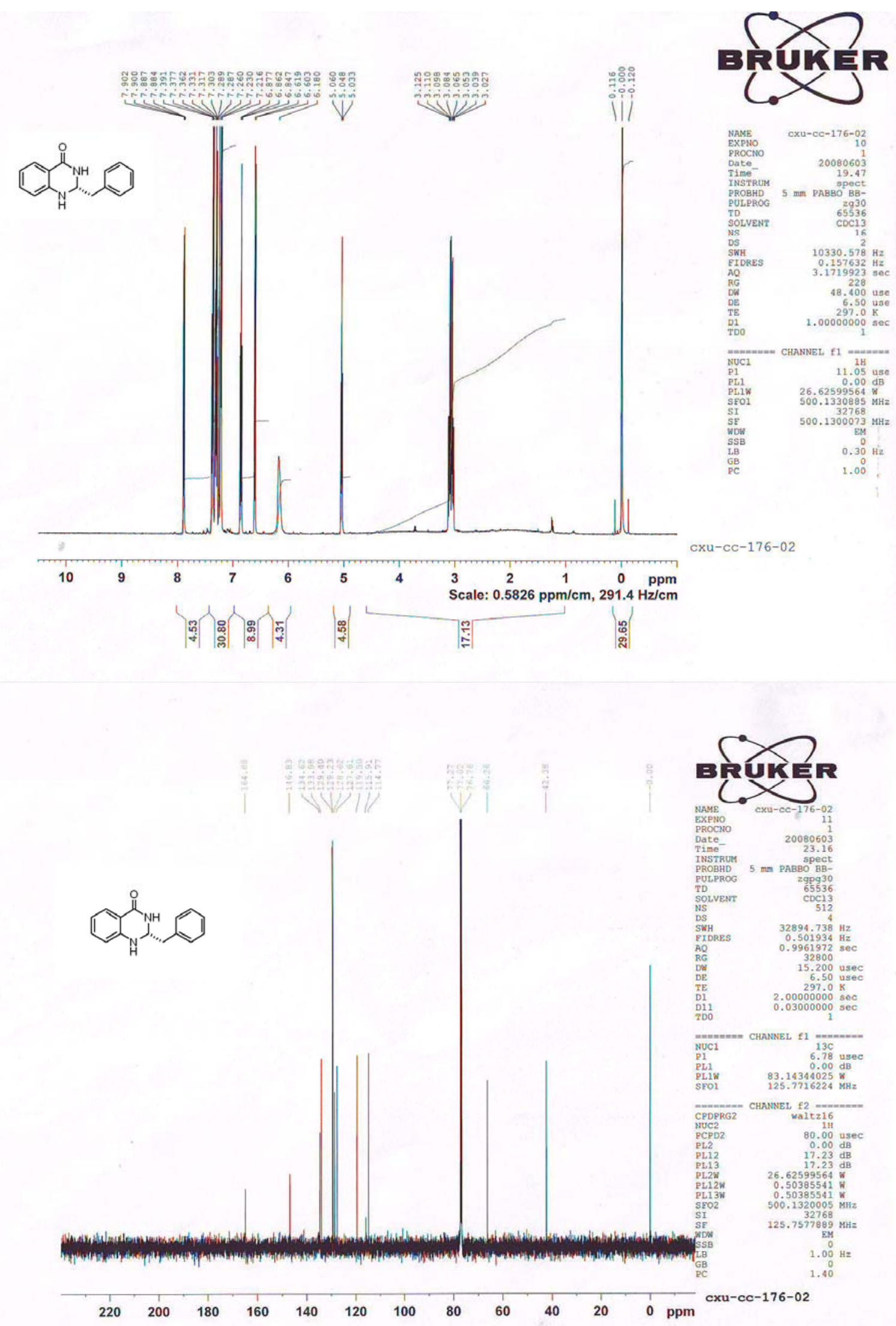

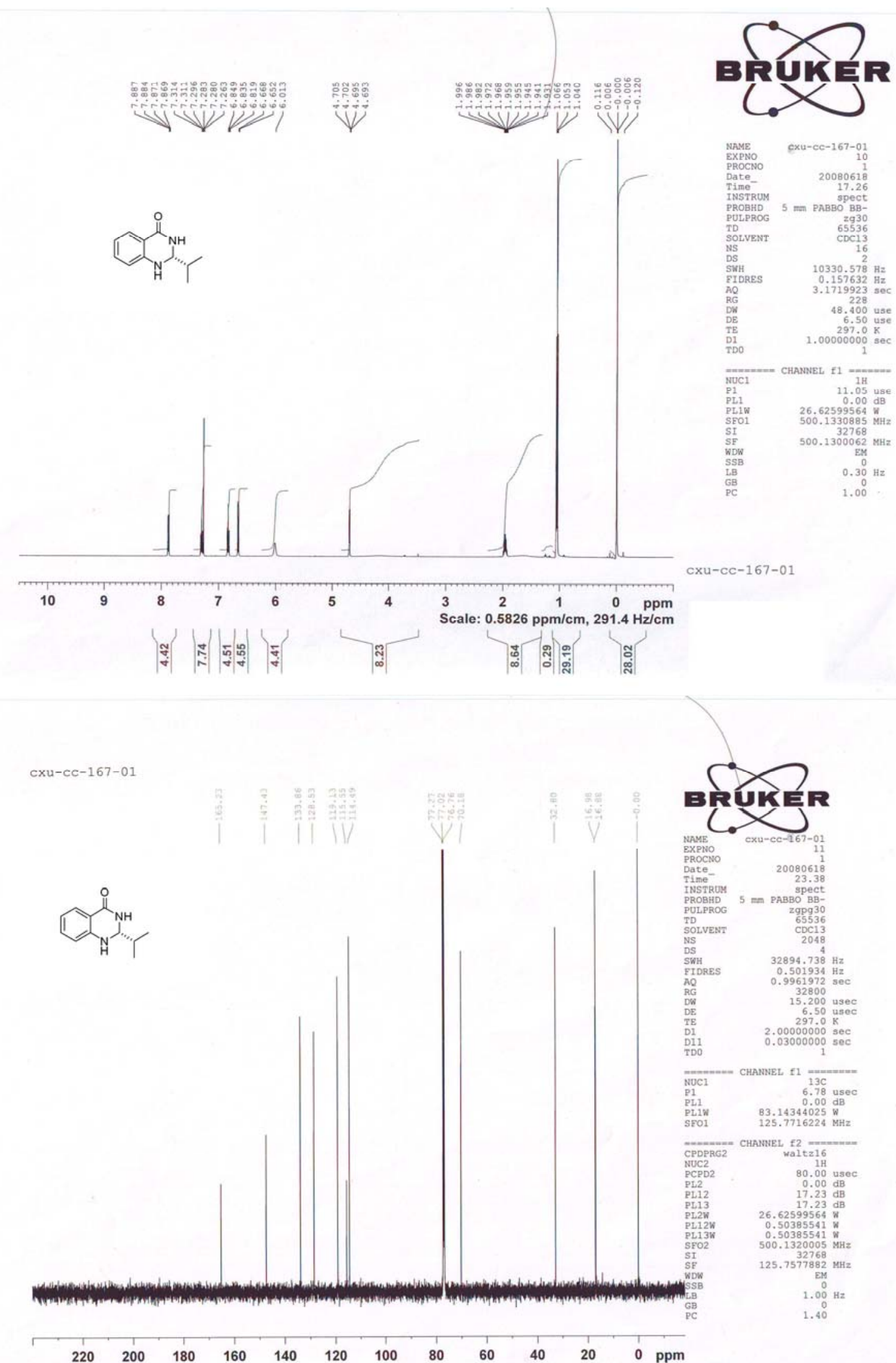

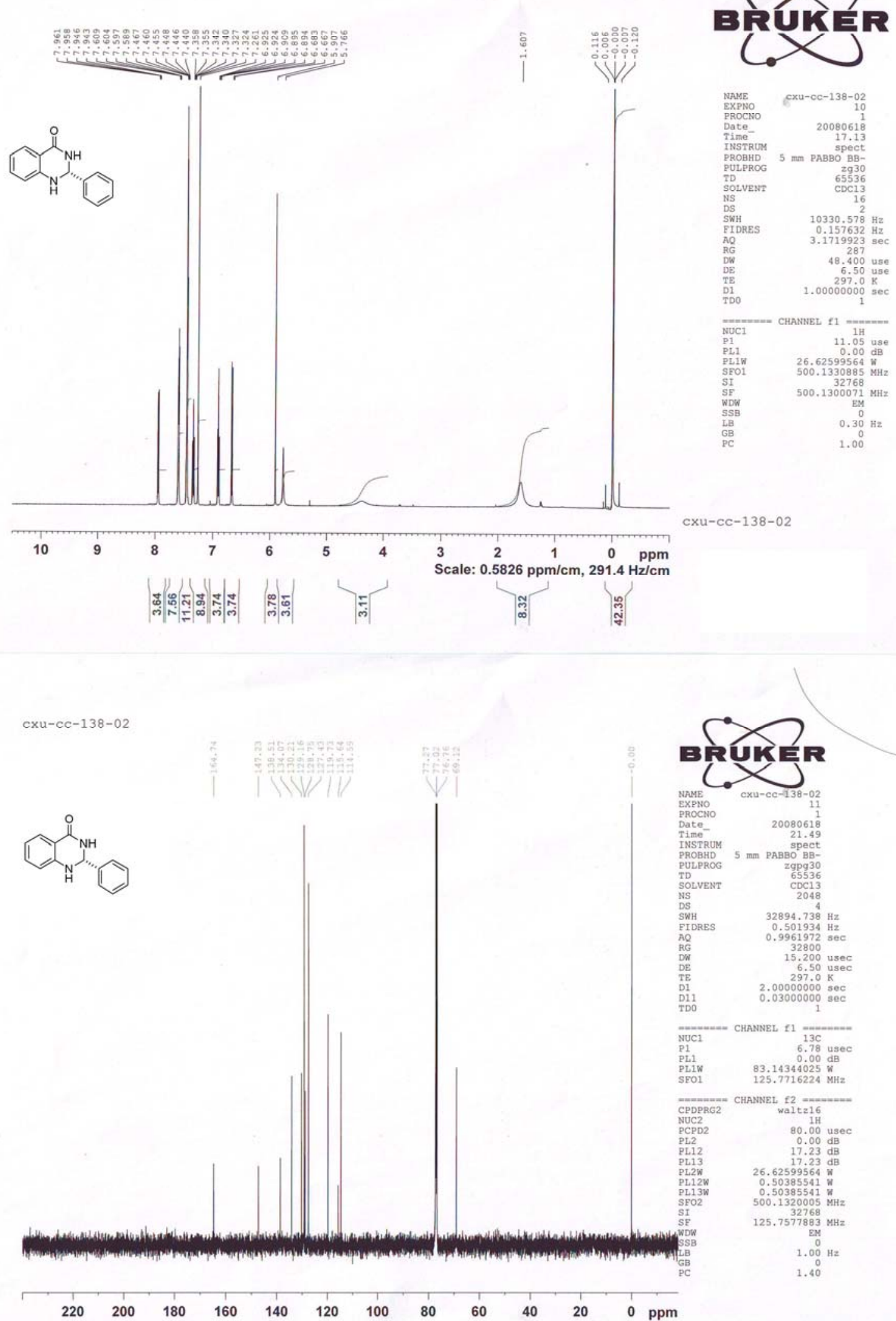

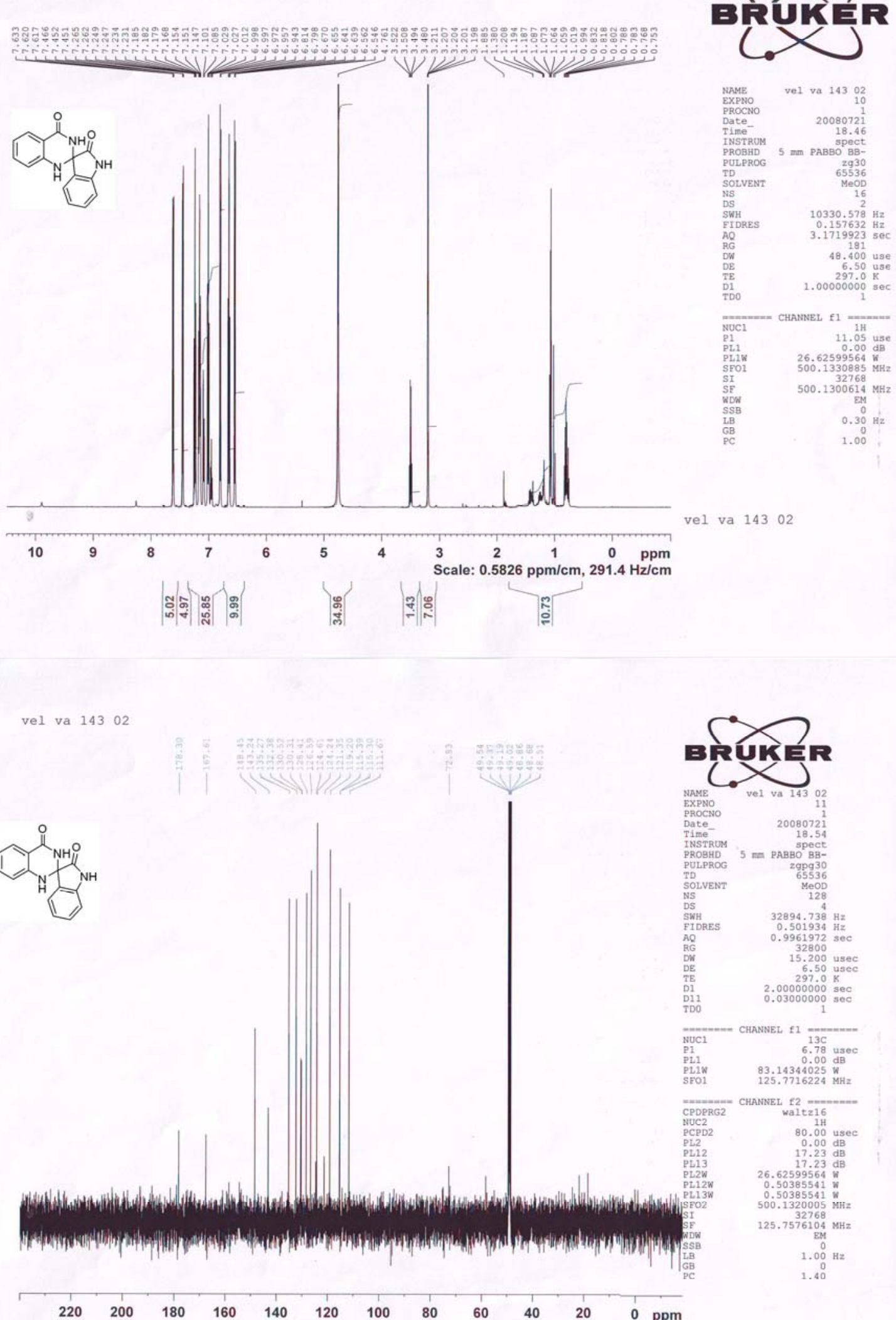

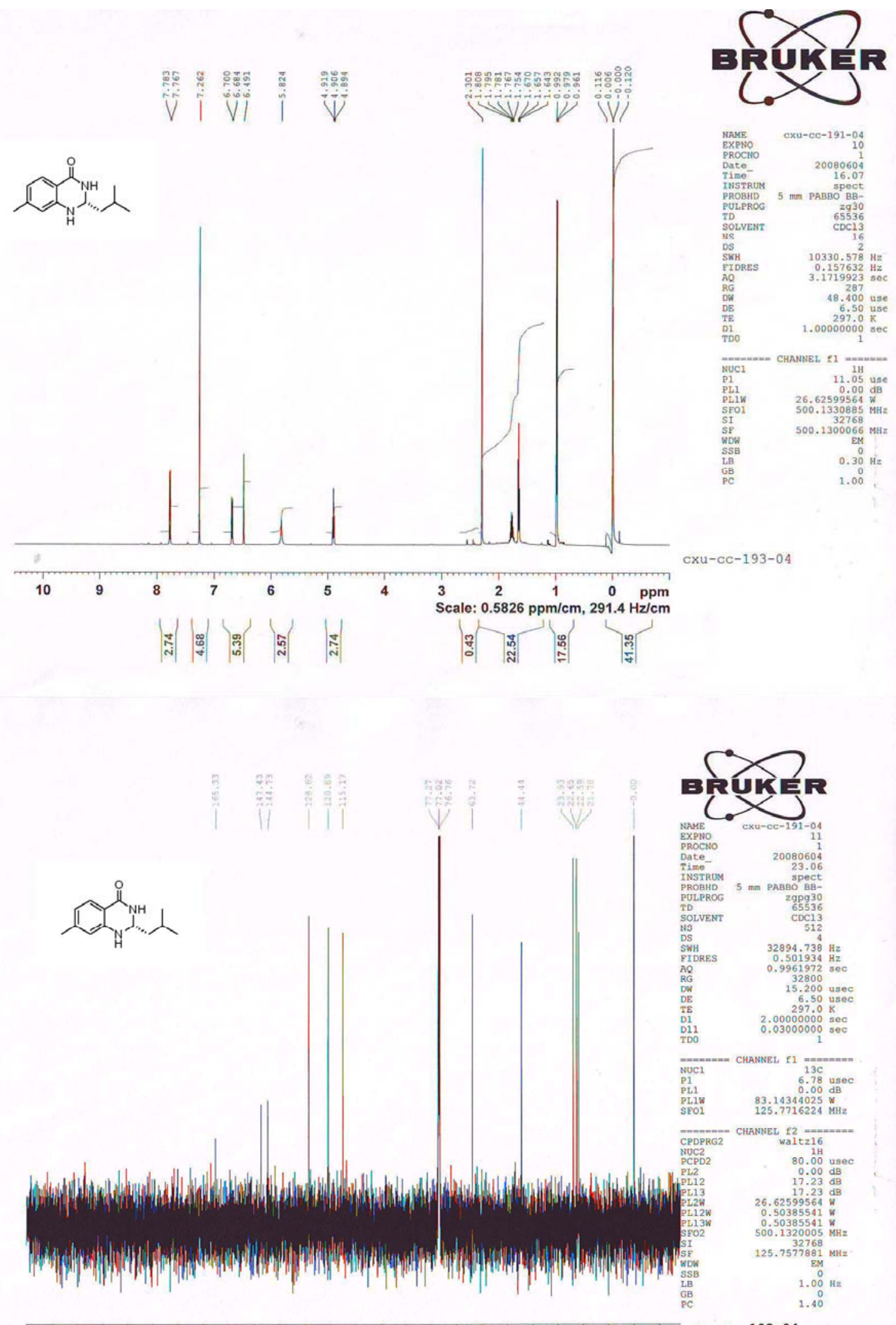

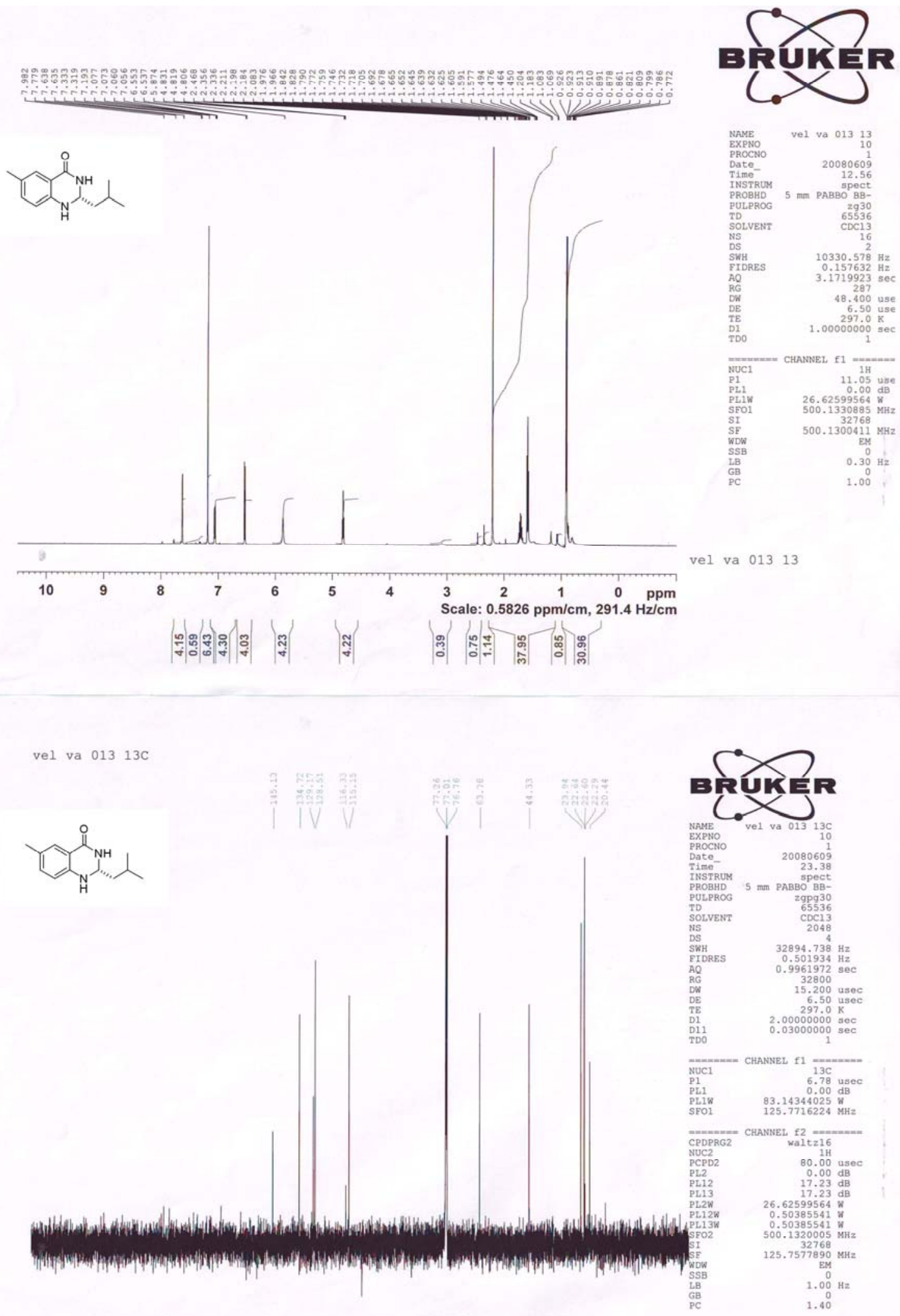

$\begin{array}{llllllllllll}220 & 200 & 180 & 160 & 140 & 120 & 100 & 80 & 60 & 40 & 20 & 0\end{array}$ 


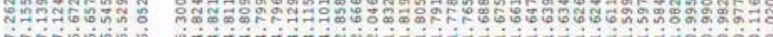

VV V V U U U V V
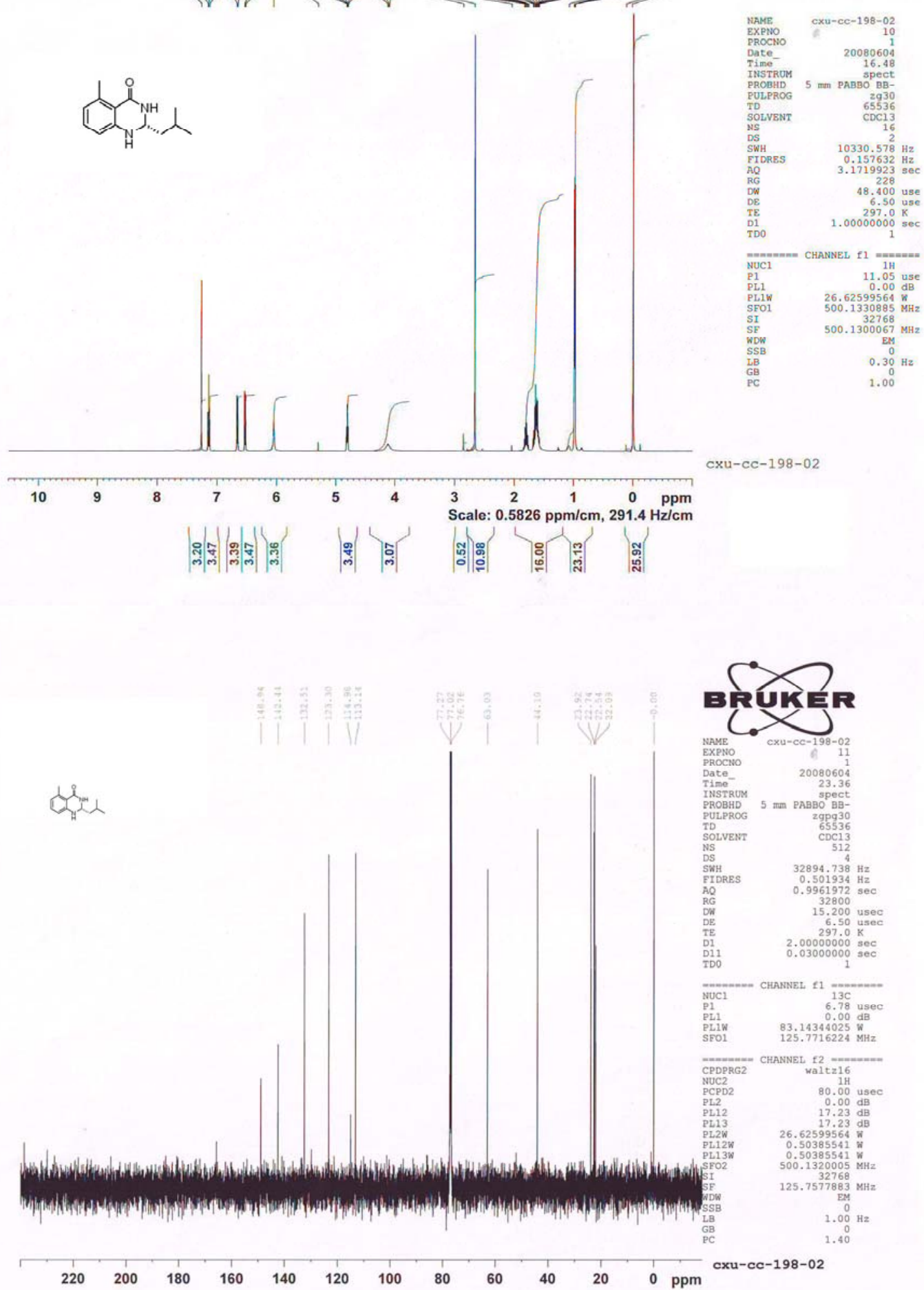

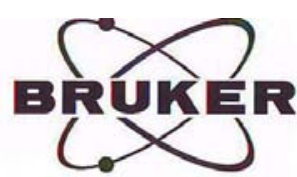

cxu-cc-198-02 

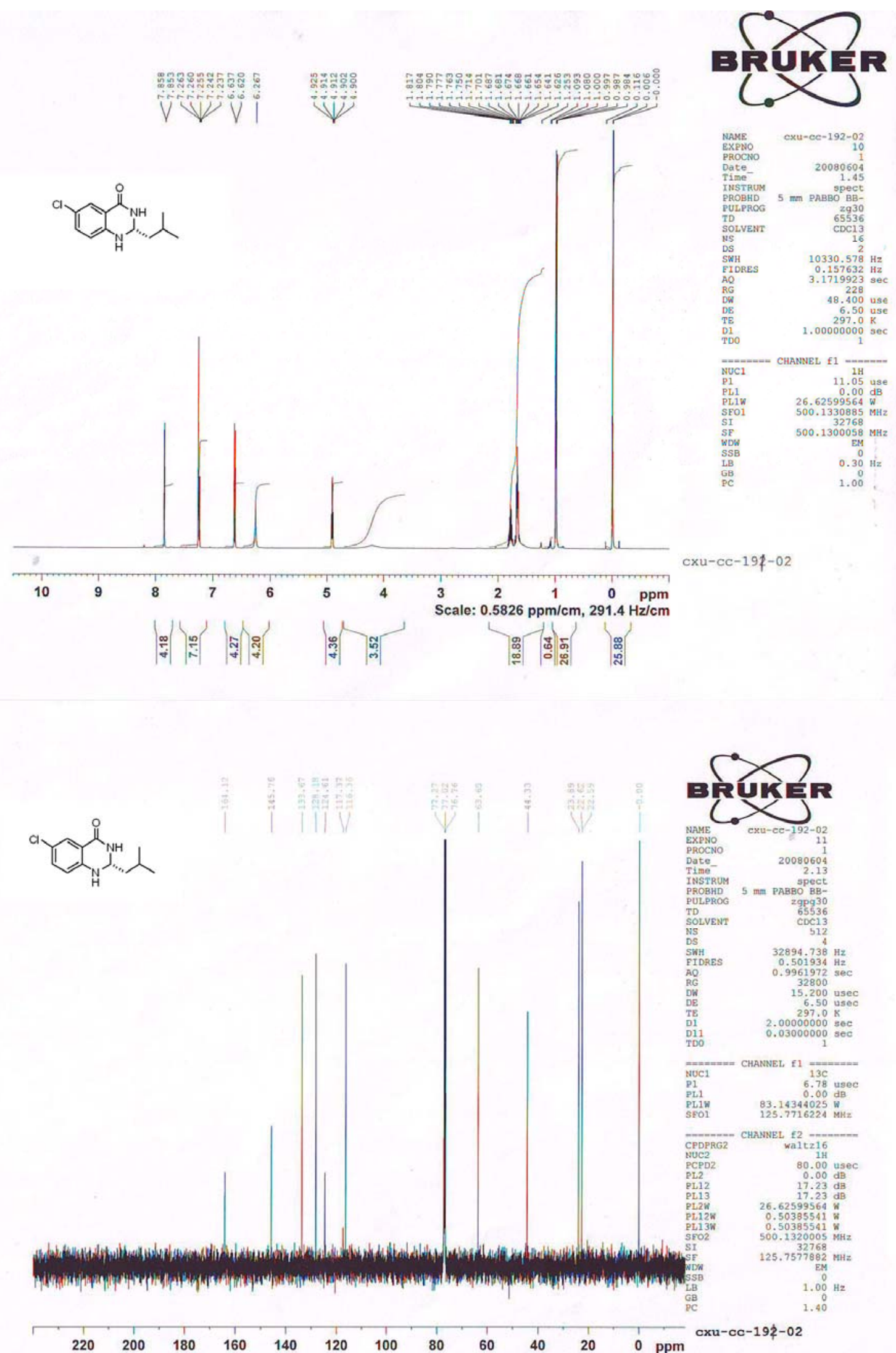

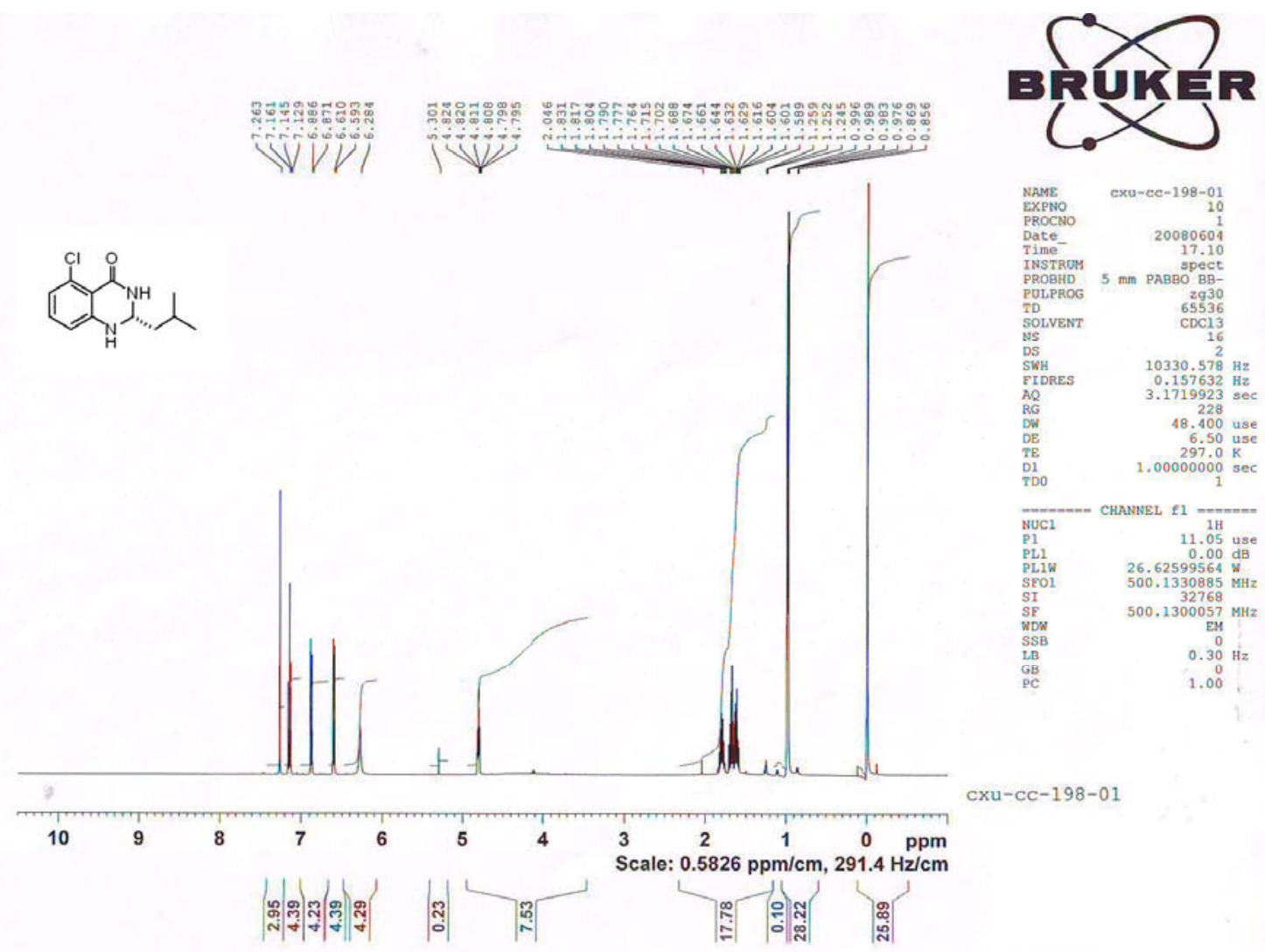

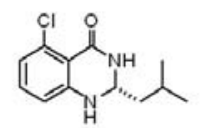
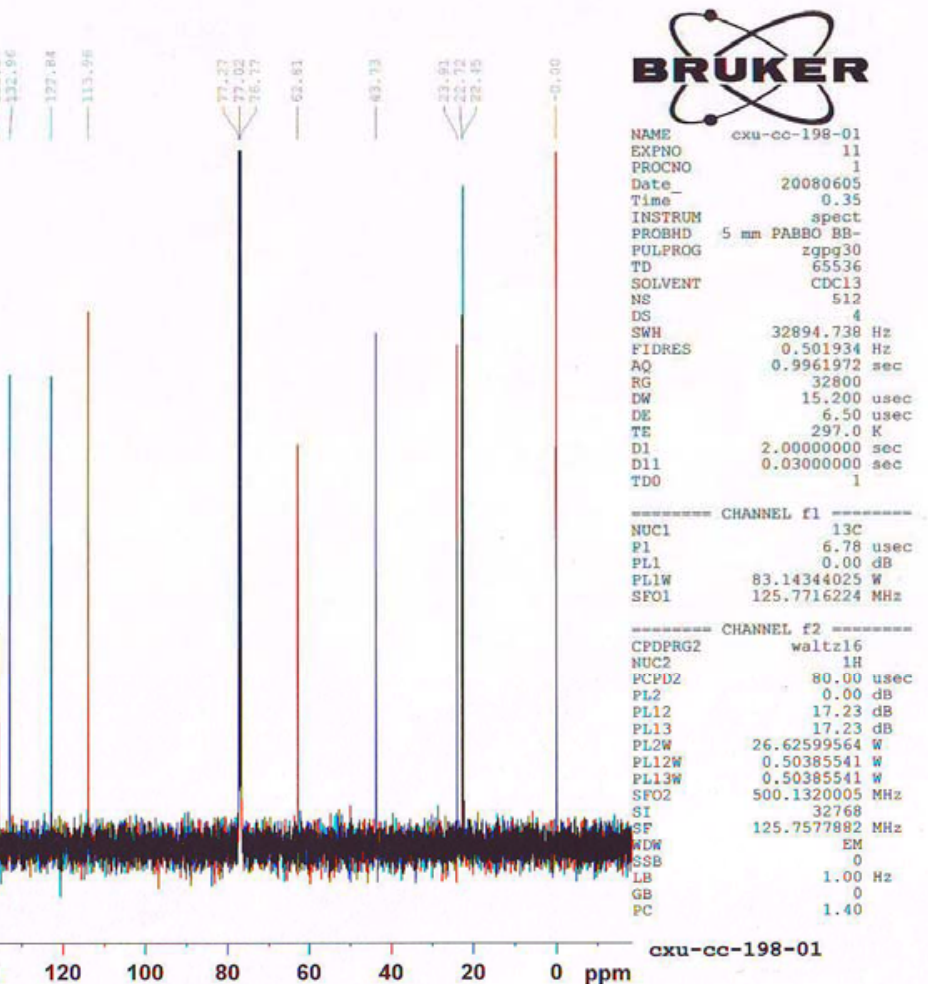

cxu-cc-198-01 

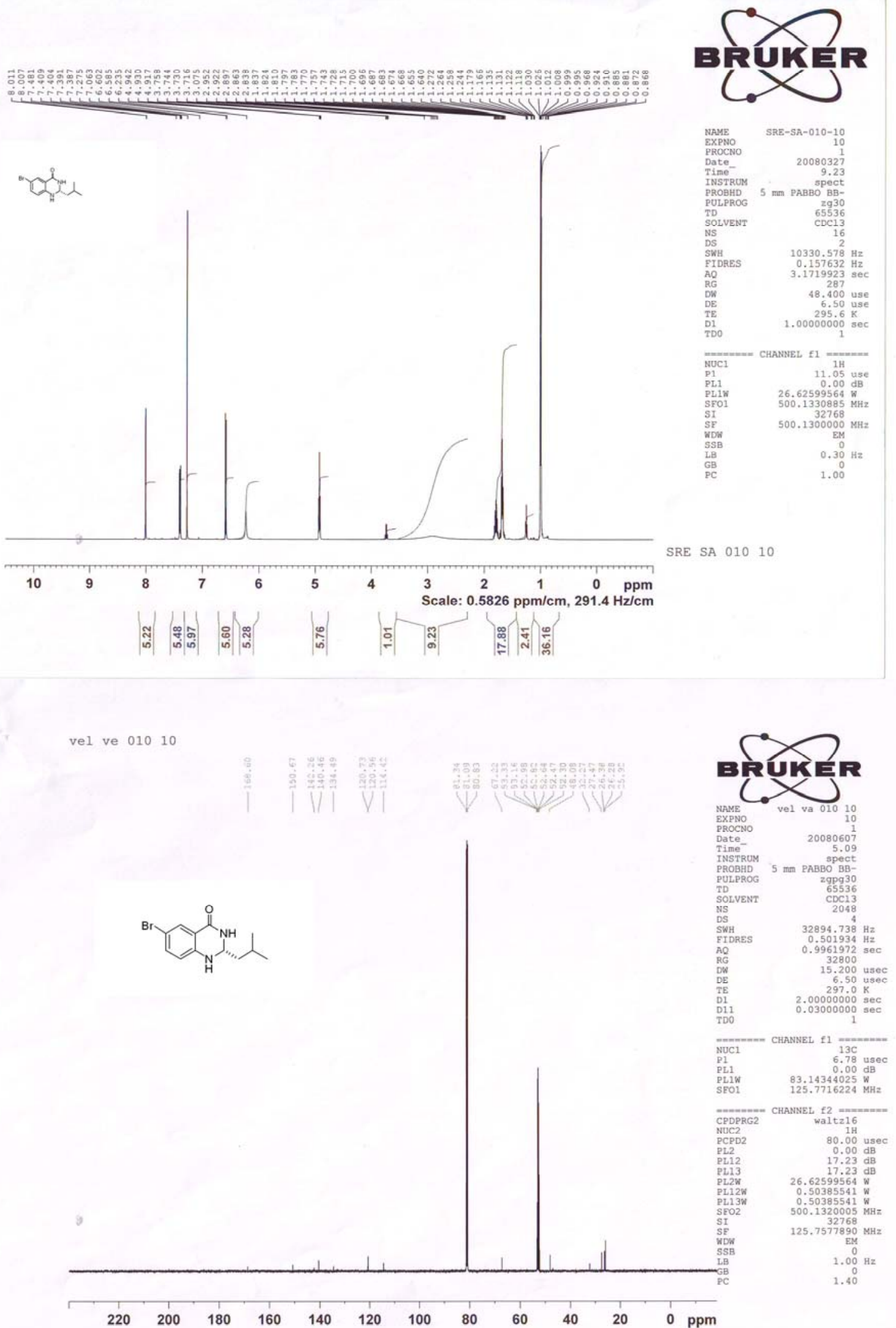

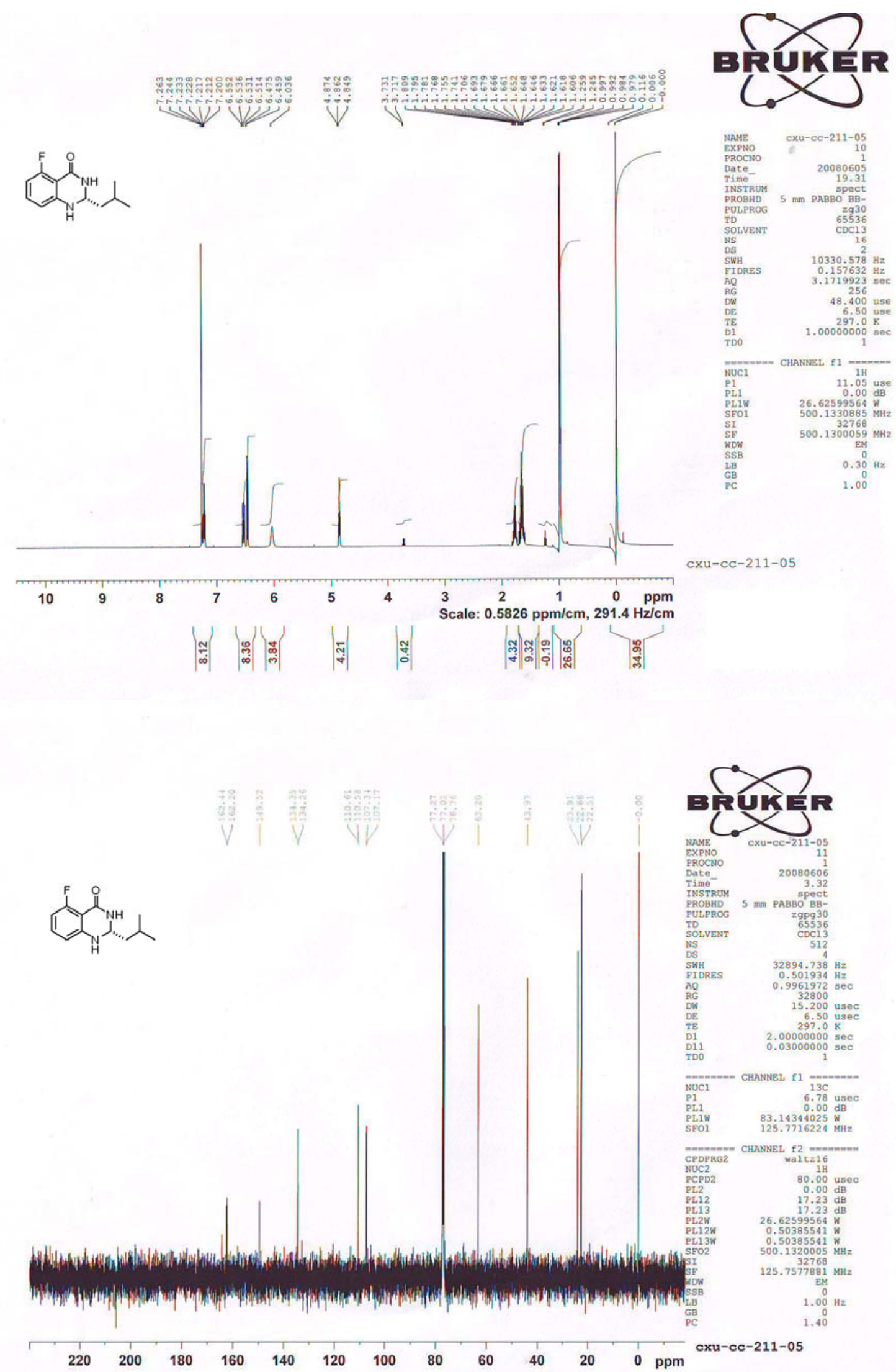


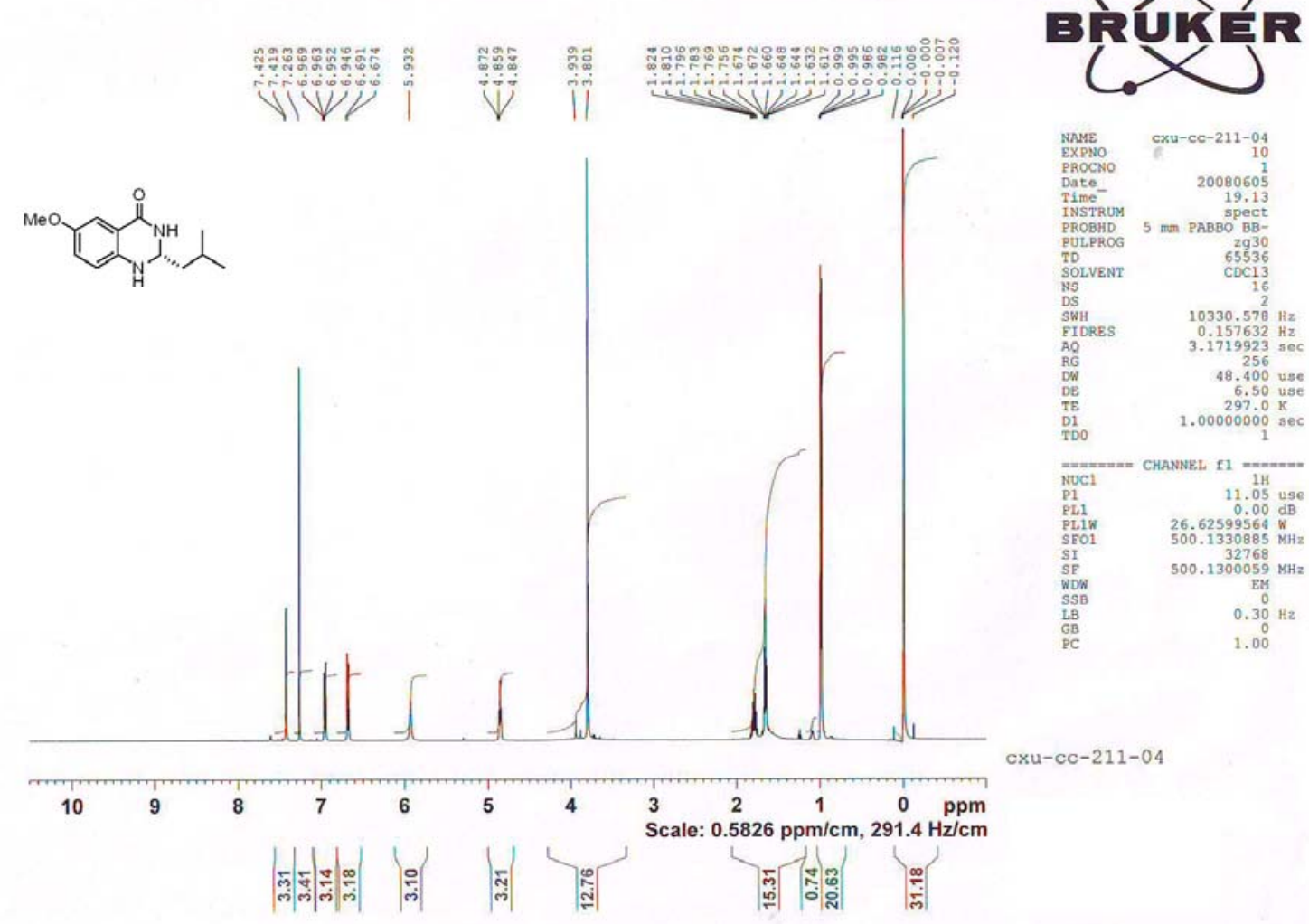

Meo
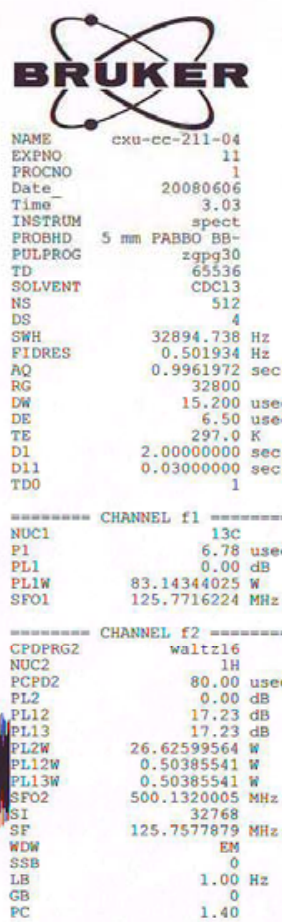

$\begin{array}{llllllllllll}220 & 200 & 180 & 160 & 140 & 120 & 100 & 80 & 60 & 40 & 20 & 0\end{array}$

cxu-cc-211-04 

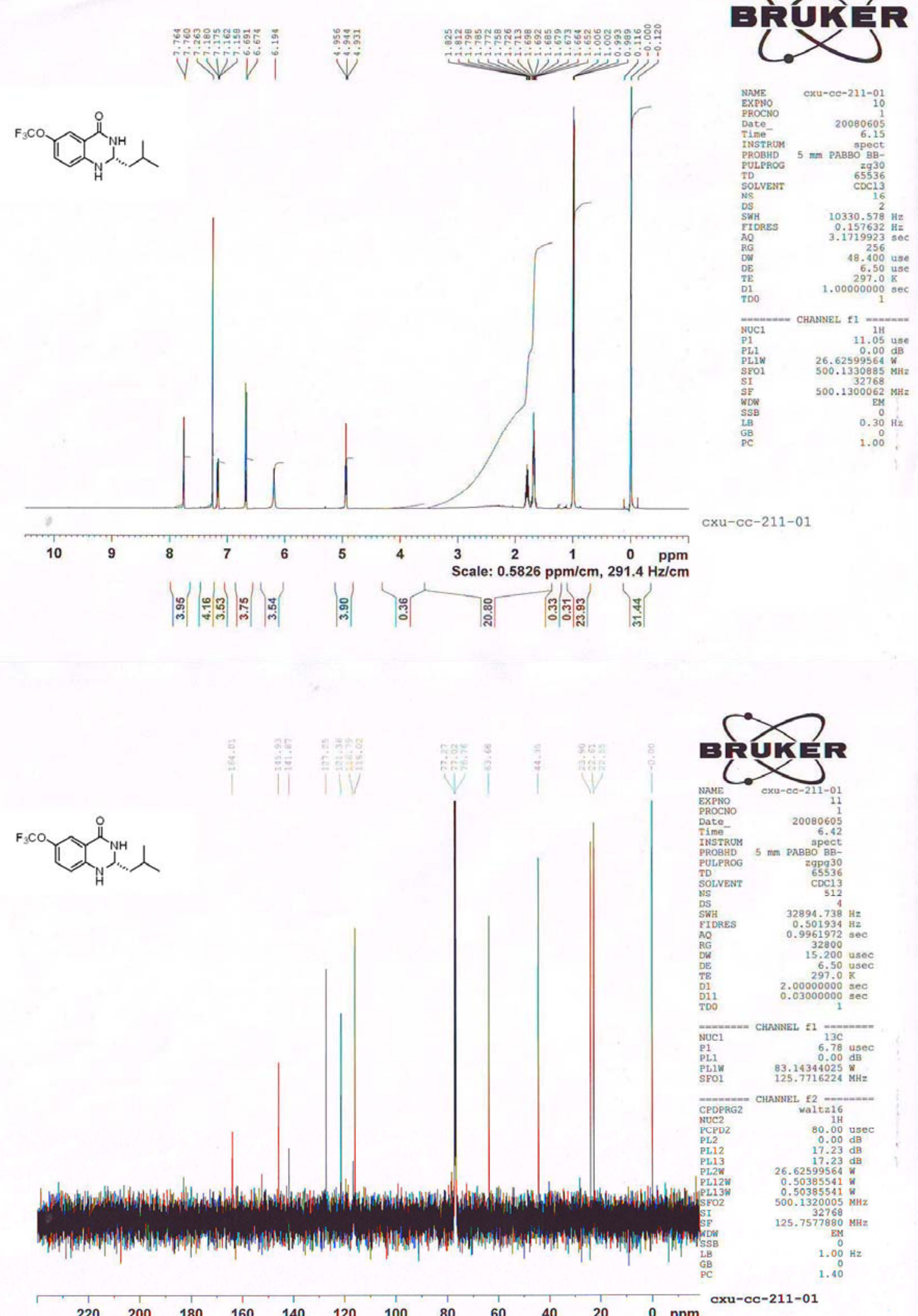


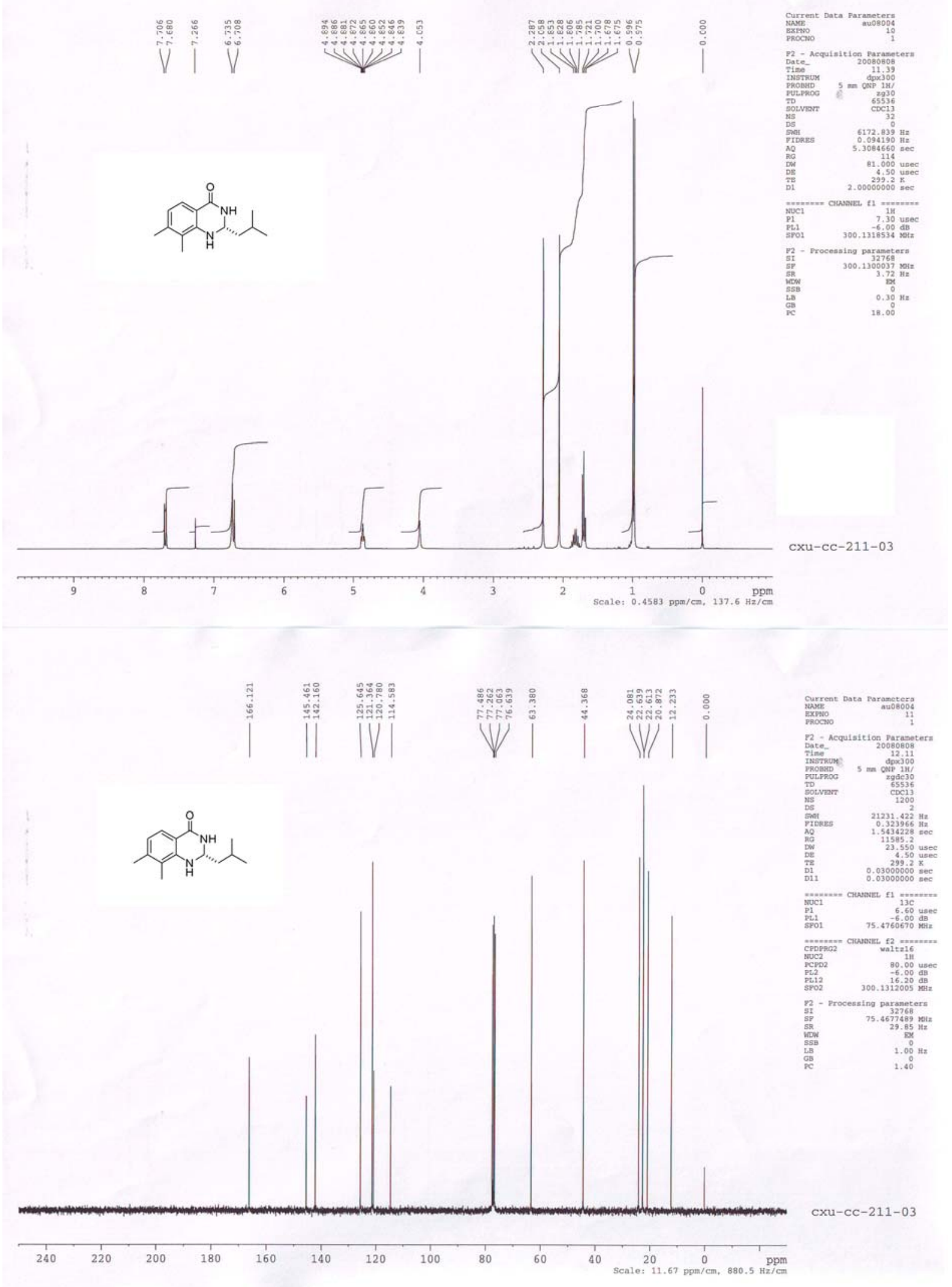



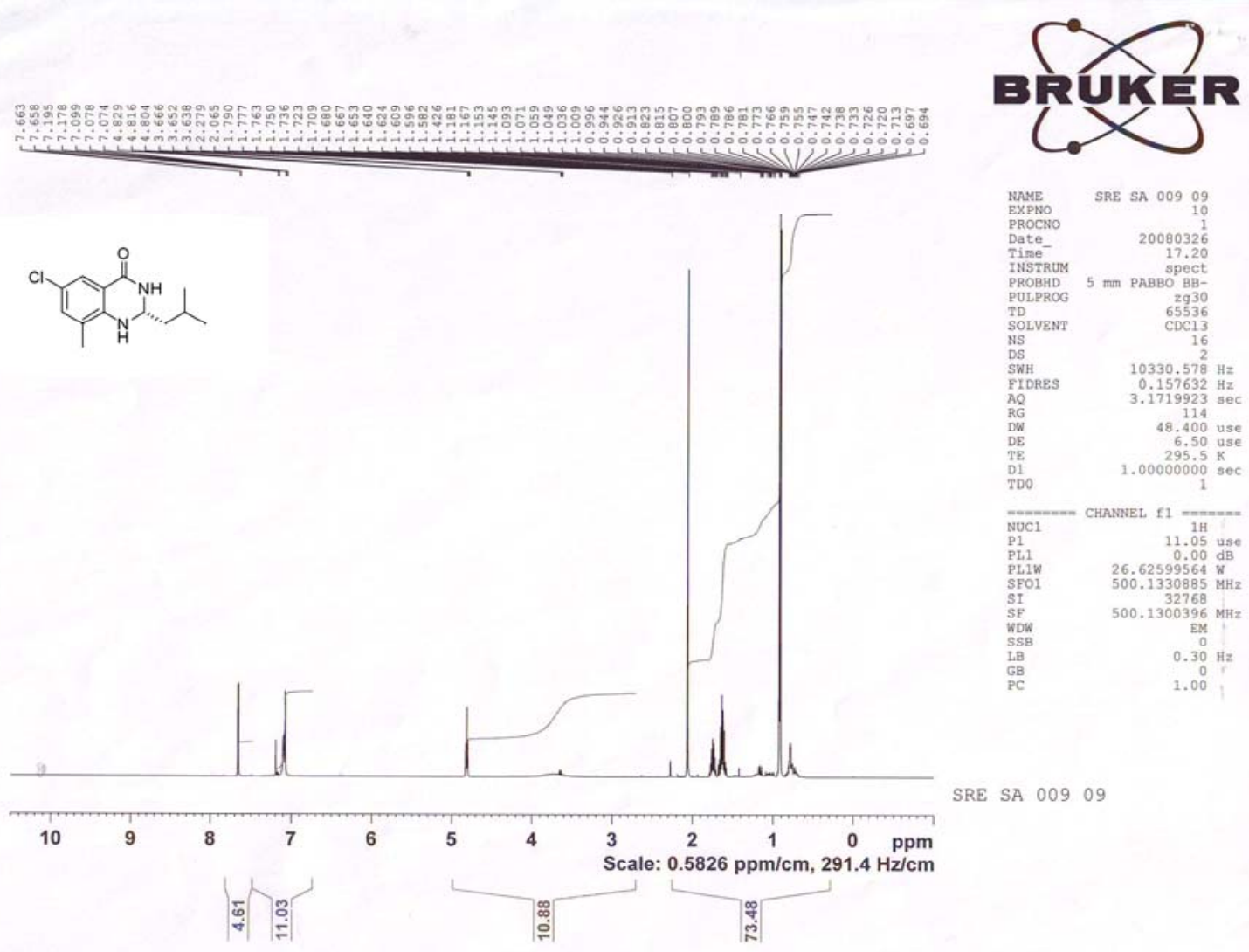

vel va $00909 \mathrm{C}$
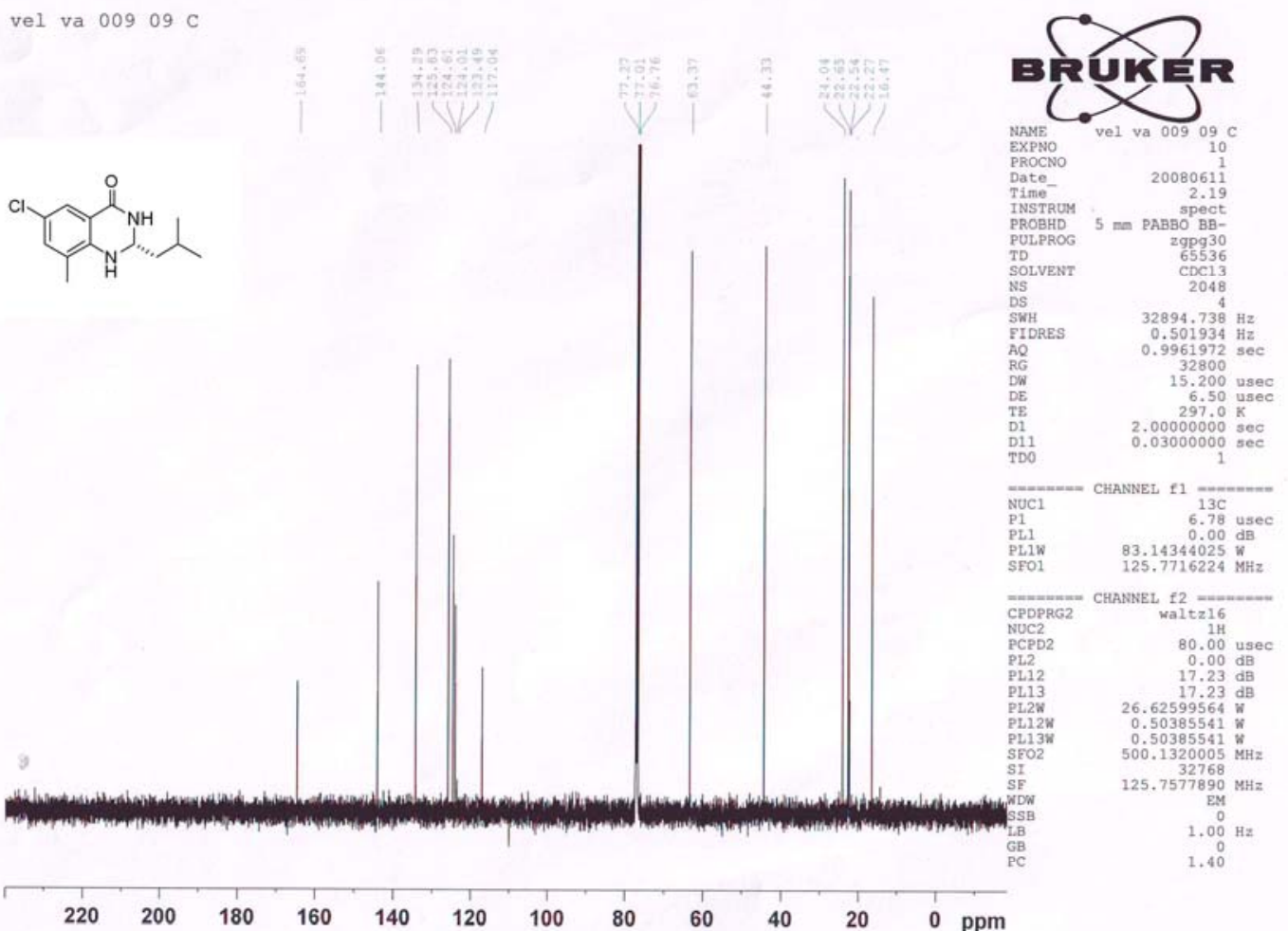

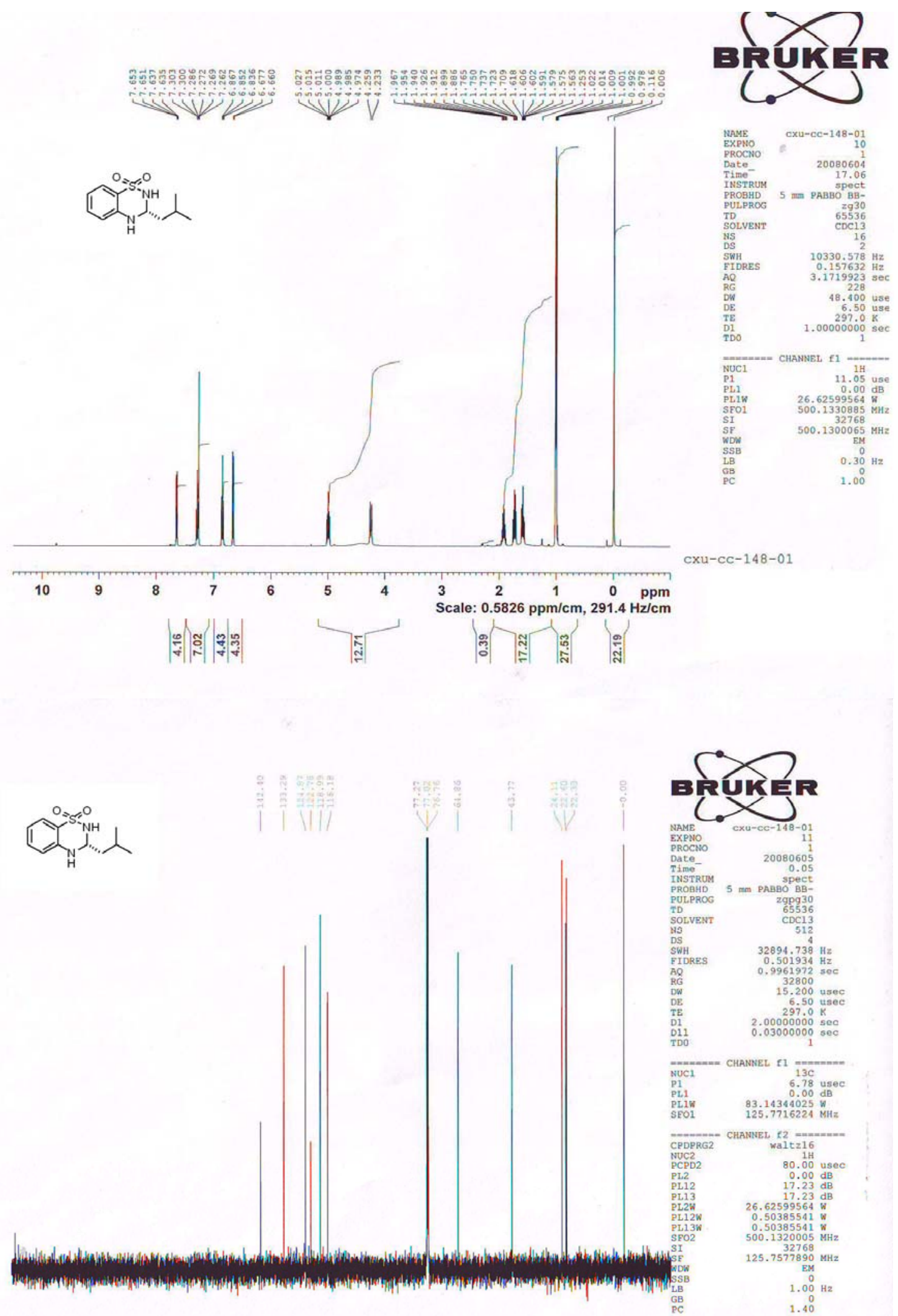

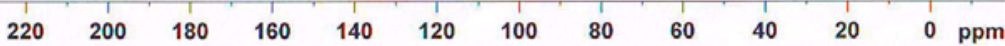

cxu-cc-148-01 

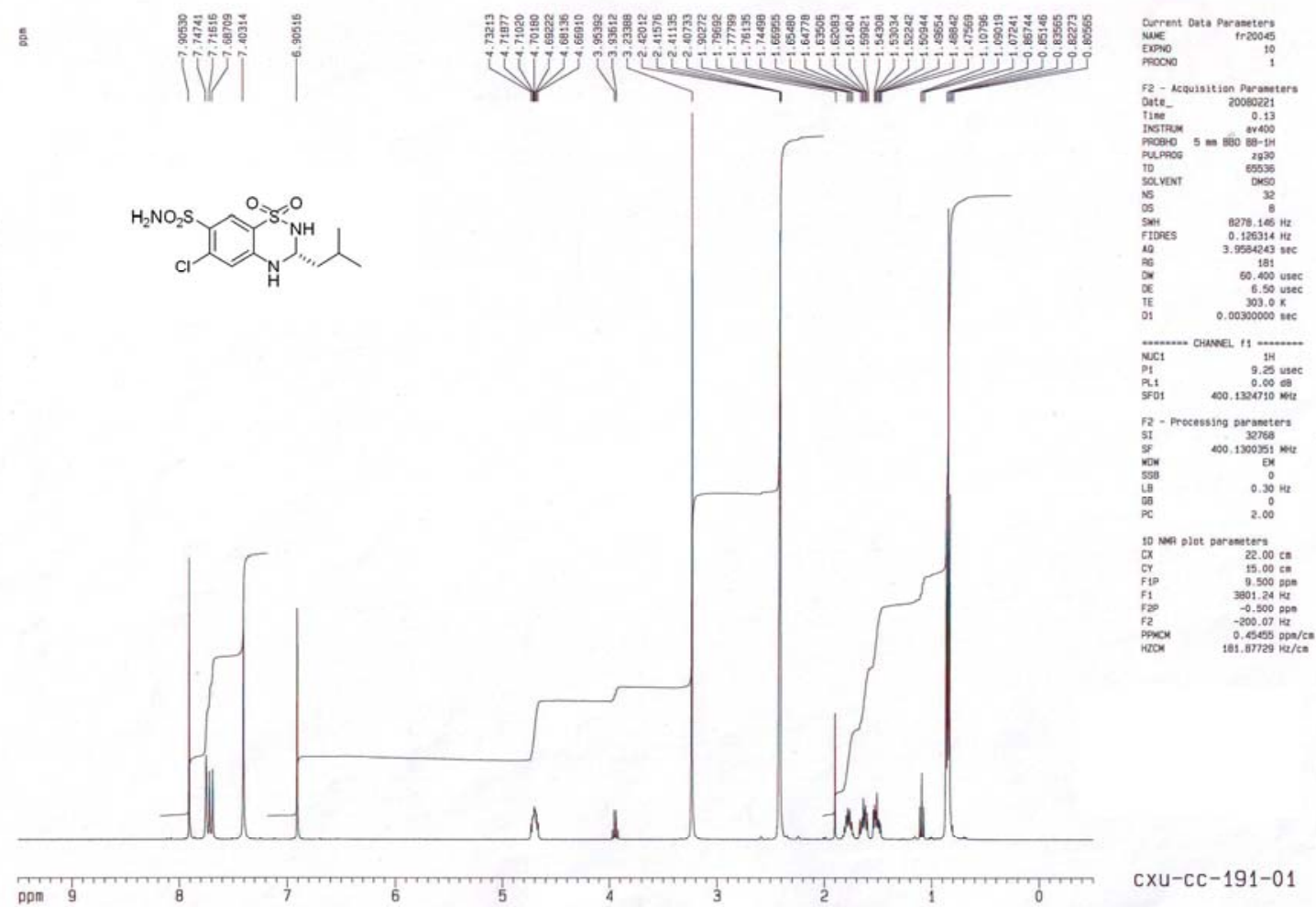

cxu-cc-191-01

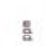<smiles>CC(C)C[C@@H]1Nc2cc(Cl)c(S(N)(=O)=O)cc2S(=O)(=O)N1</smiles>

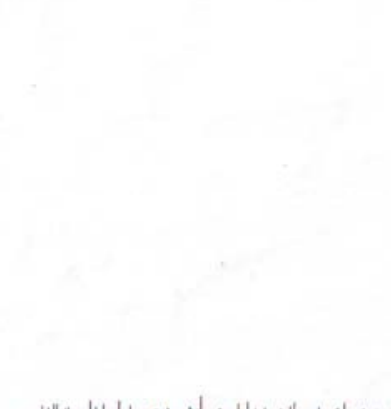

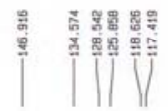

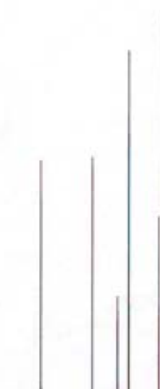

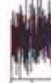

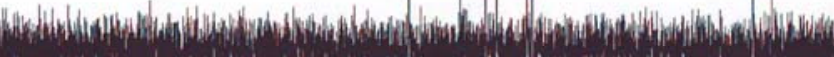

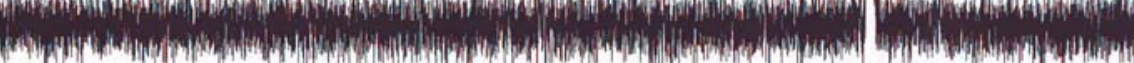
ppm 200

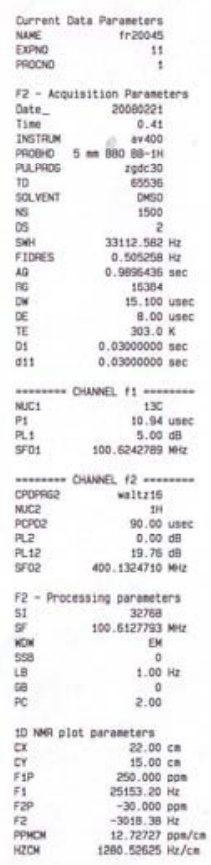

cxu-cc-191-01 

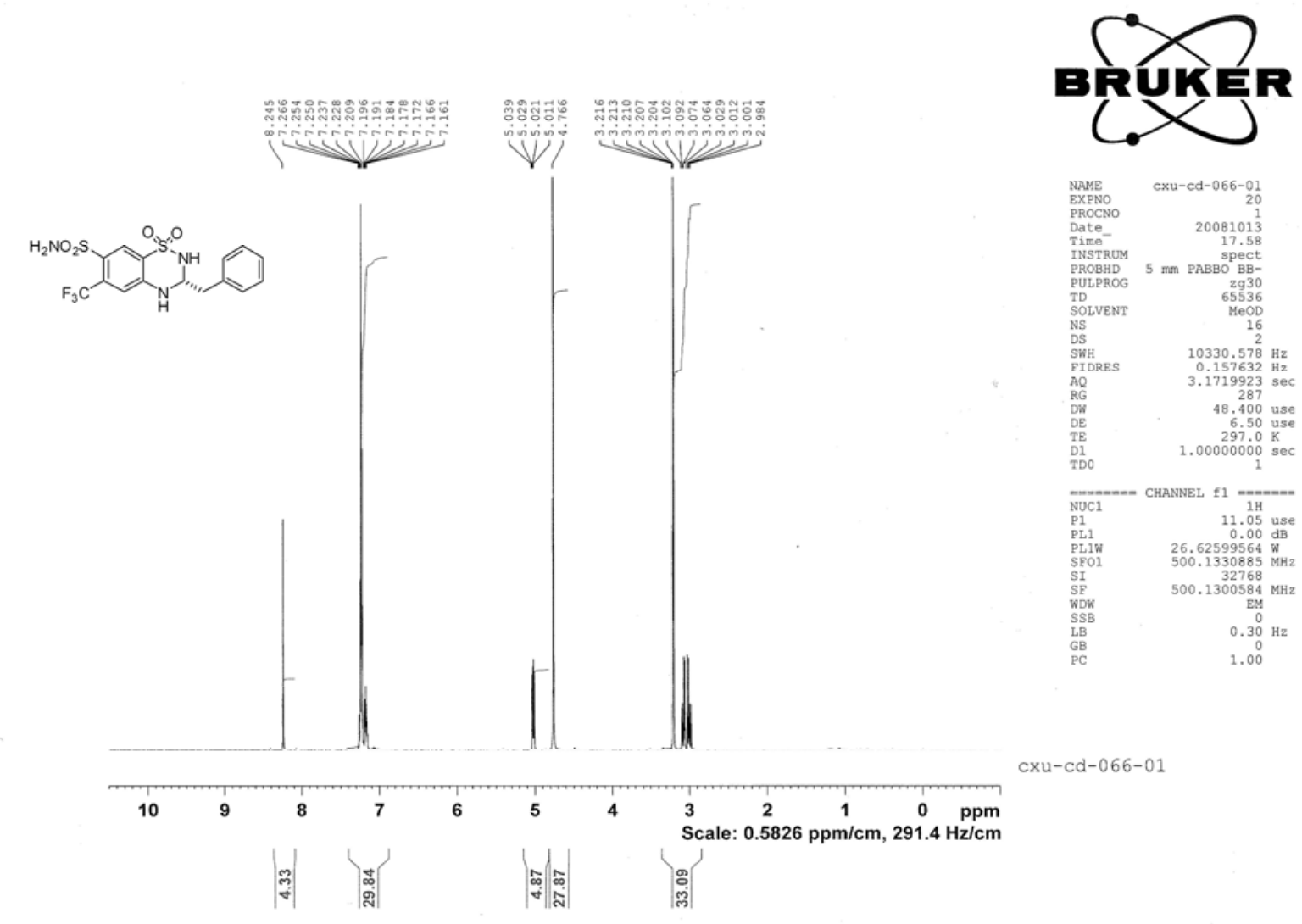

cxu-cd-066-01
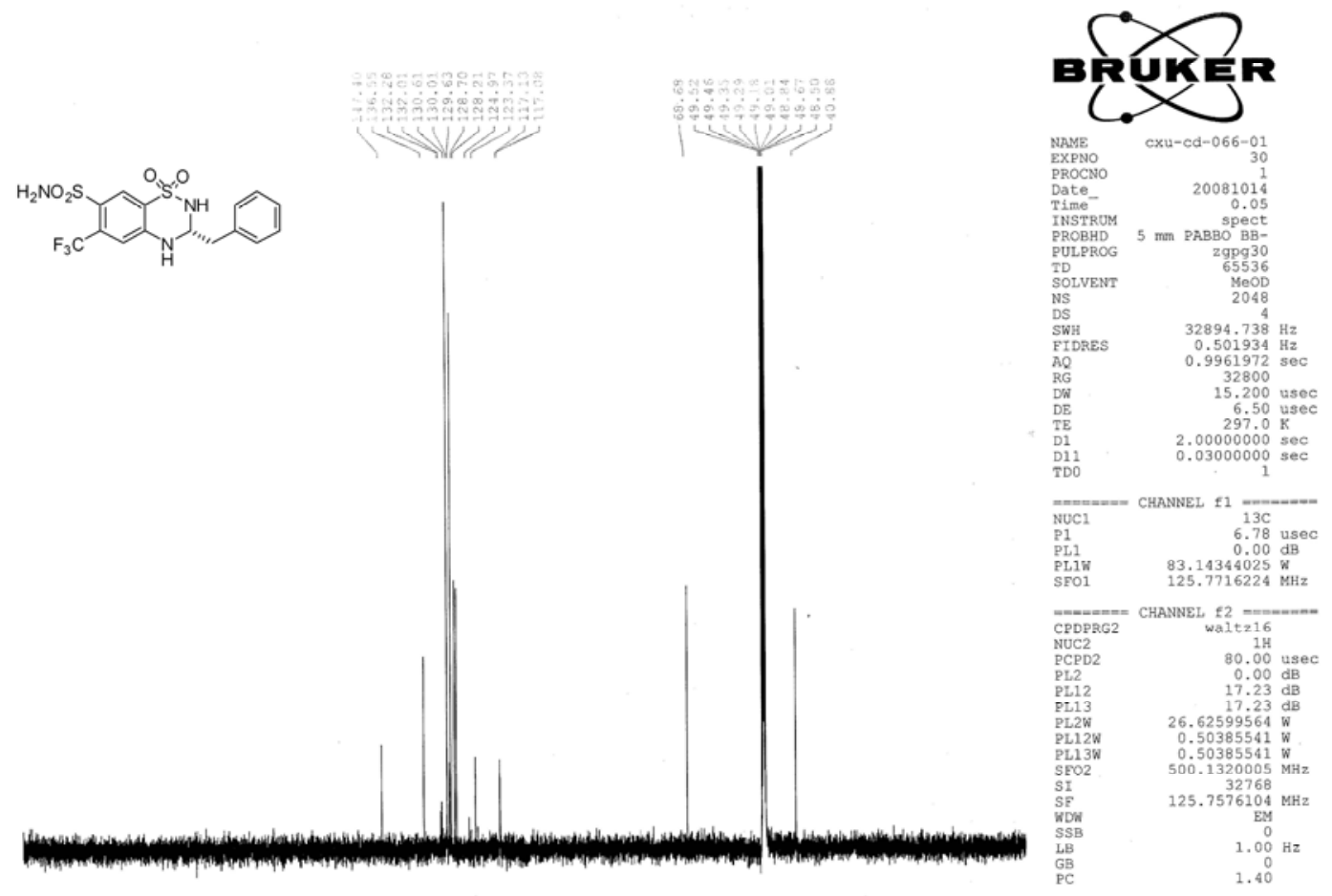

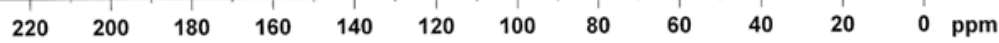

cxu-cd-066-01 


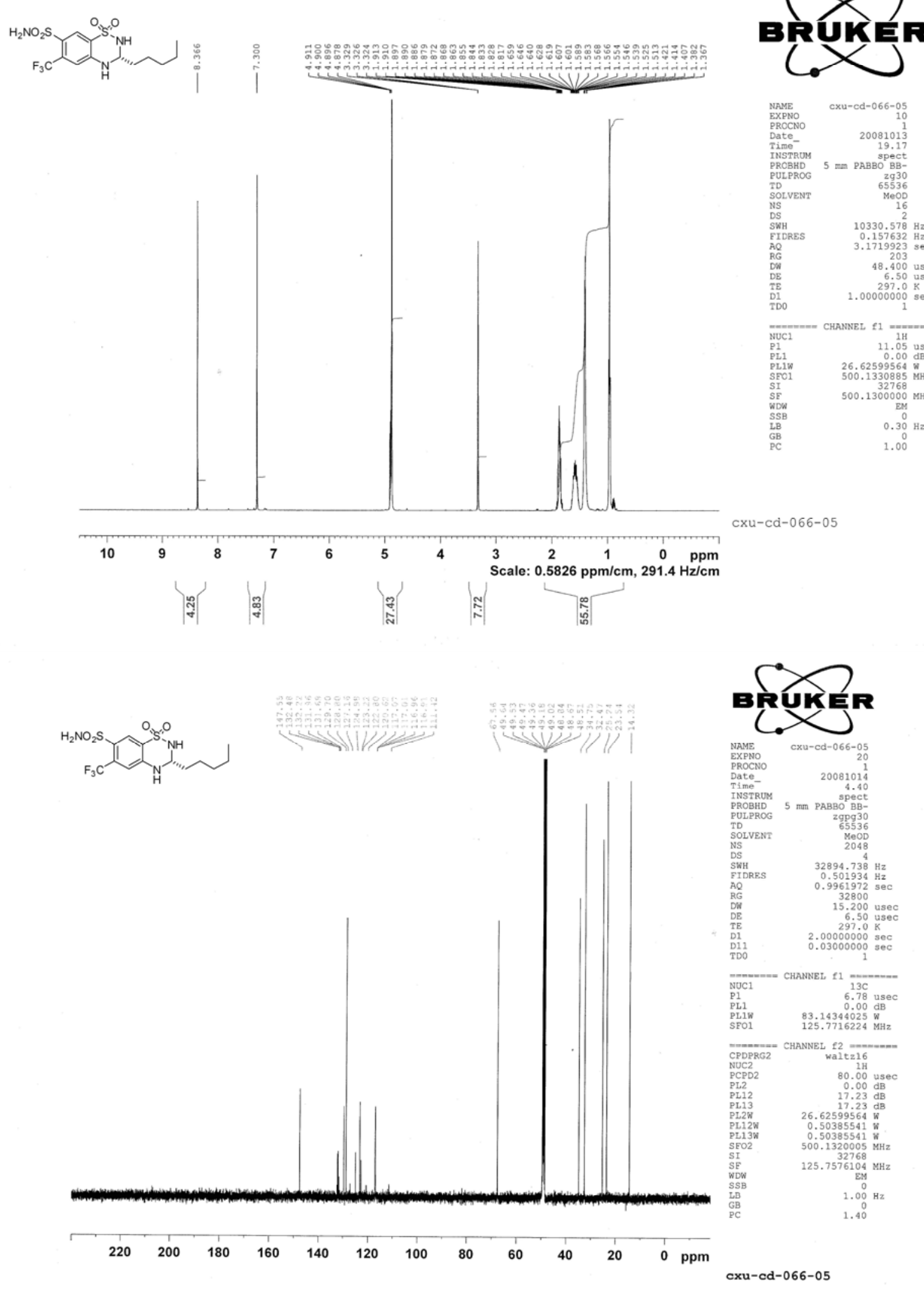



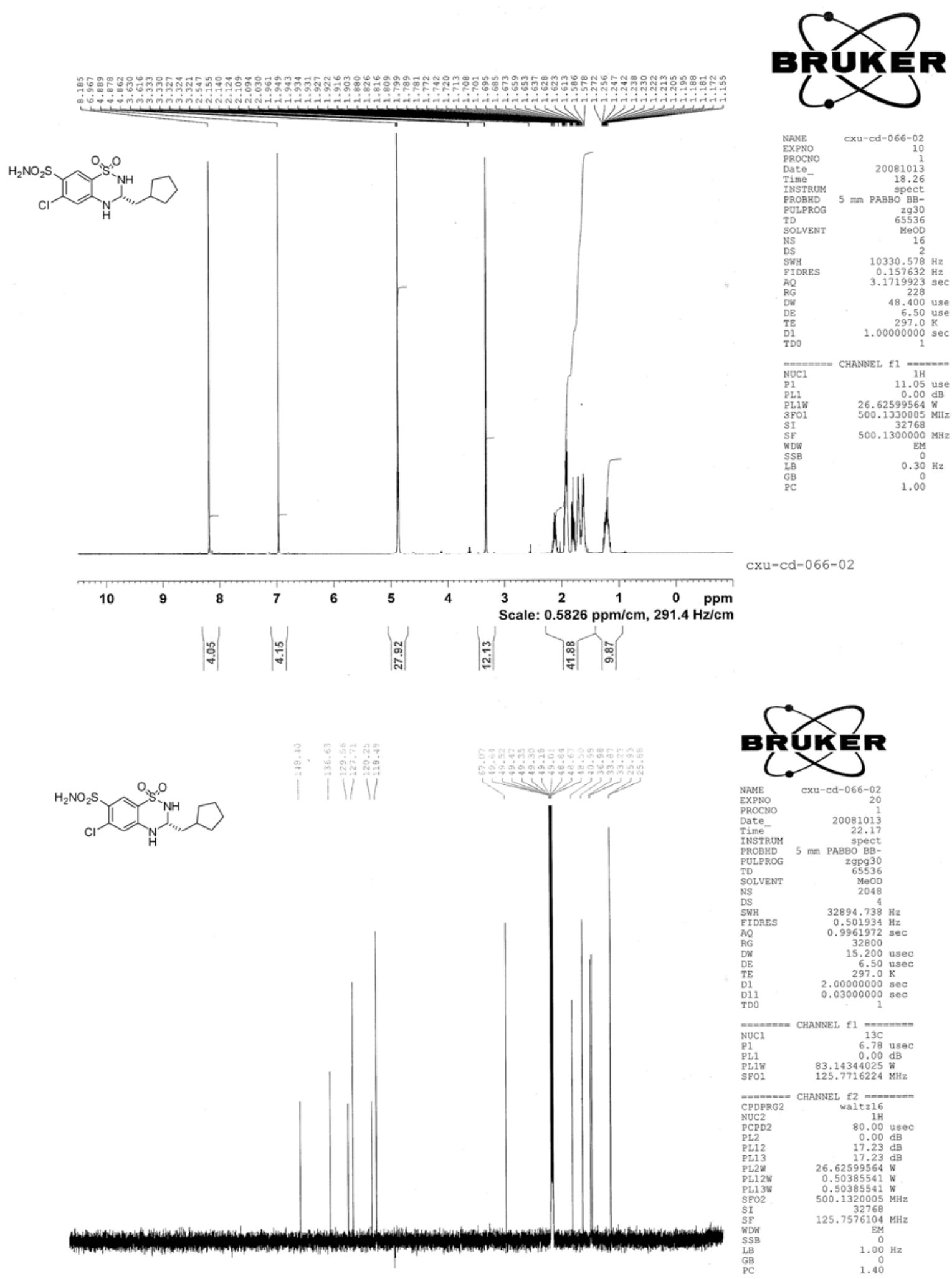

$\begin{array}{lllllllllllll}220 & 200 & 180 & 160 & 140 & 120 & 100 & 80 & 60 & 40 & 20 & 0 & \text { ppm }\end{array}$

cxu-cd-066-02 

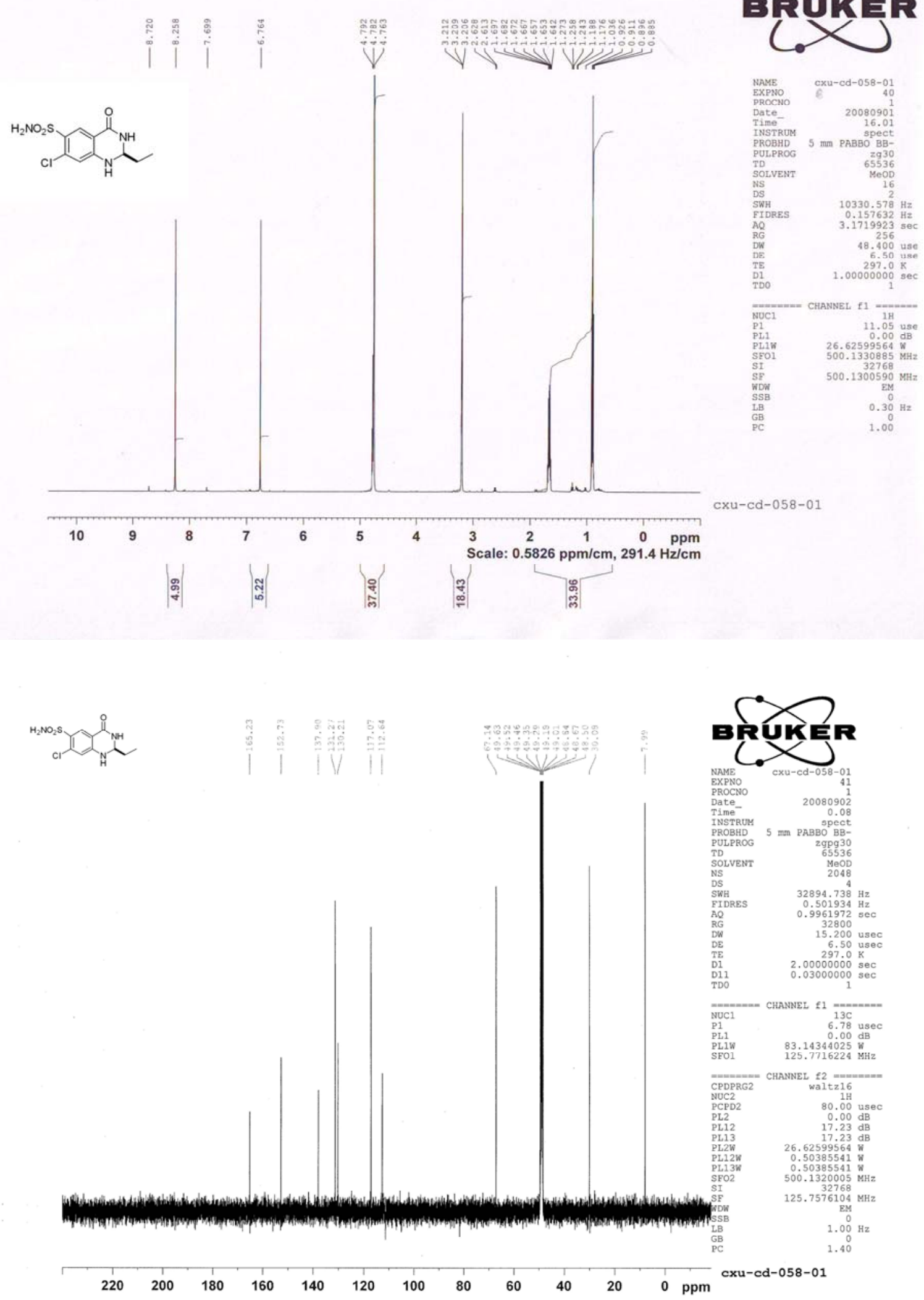


\subsection{Catalysts}

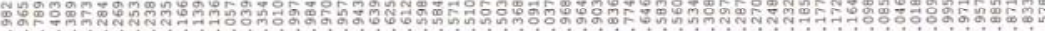
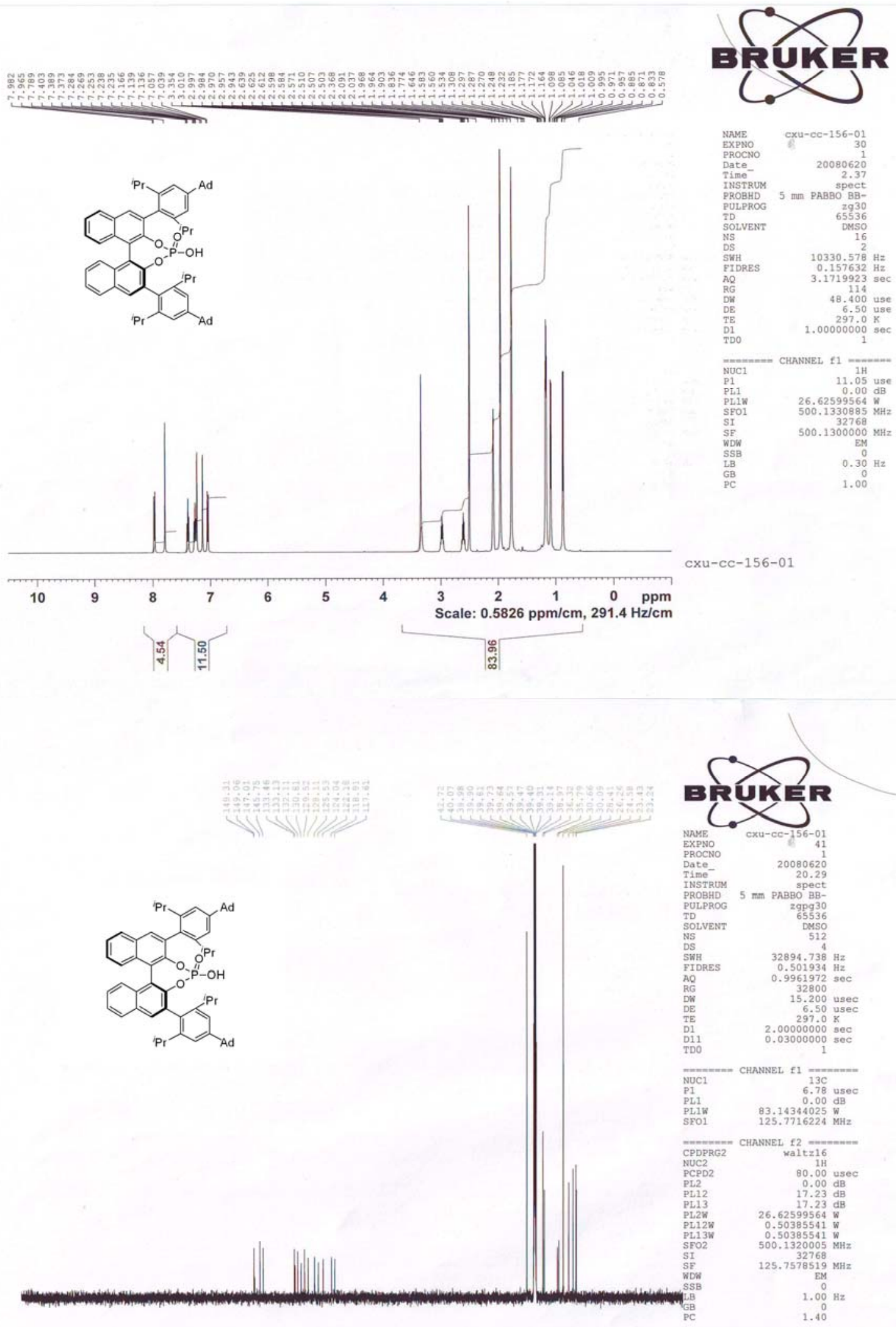

$\begin{array}{lllllllllllll}220 & 200 & 180 & 160 & 140 & 120 & 100 & 80 & 60 & 40 & 20 & 0 & \text { ppm }\end{array}$ 

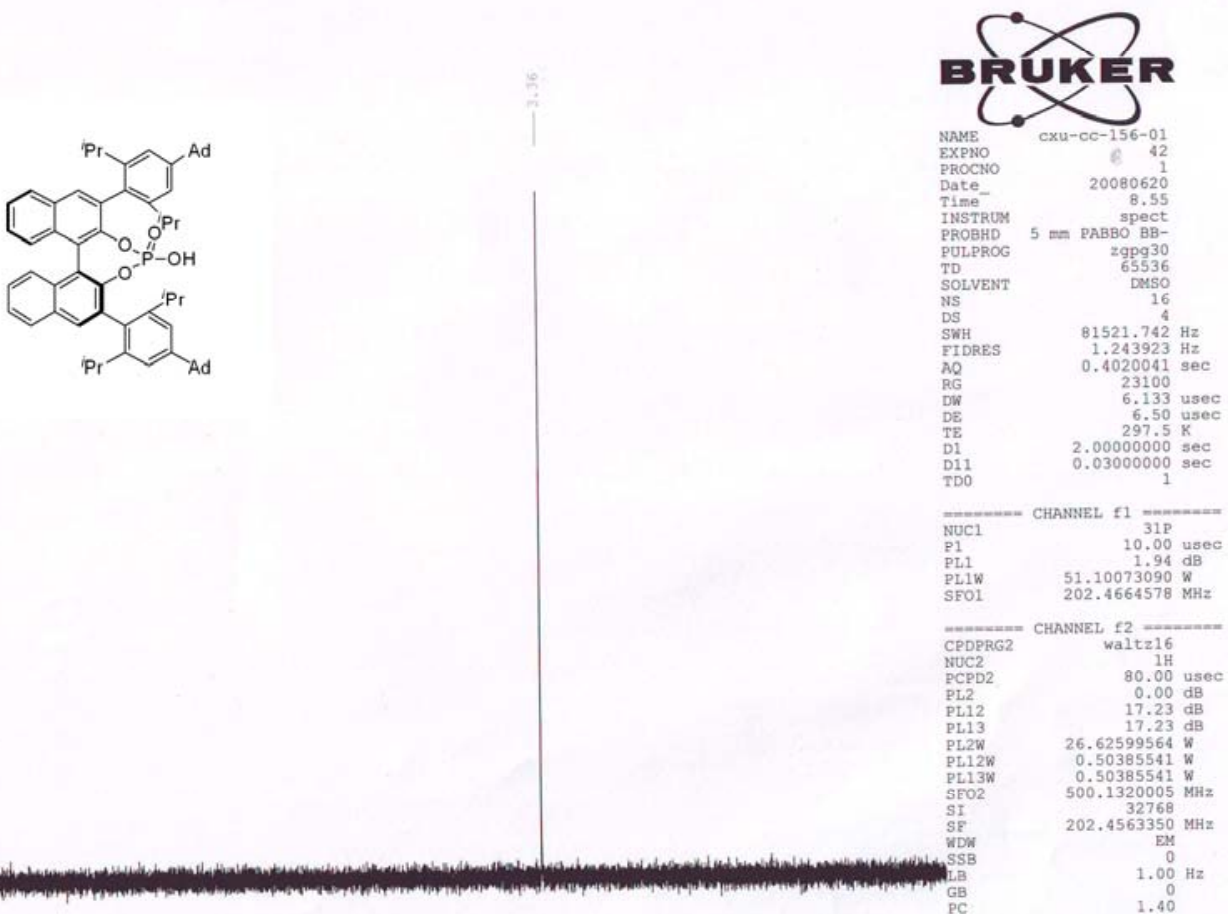

200

150

$-50$

$-100$ ppm 


\section{HPLC Traces}

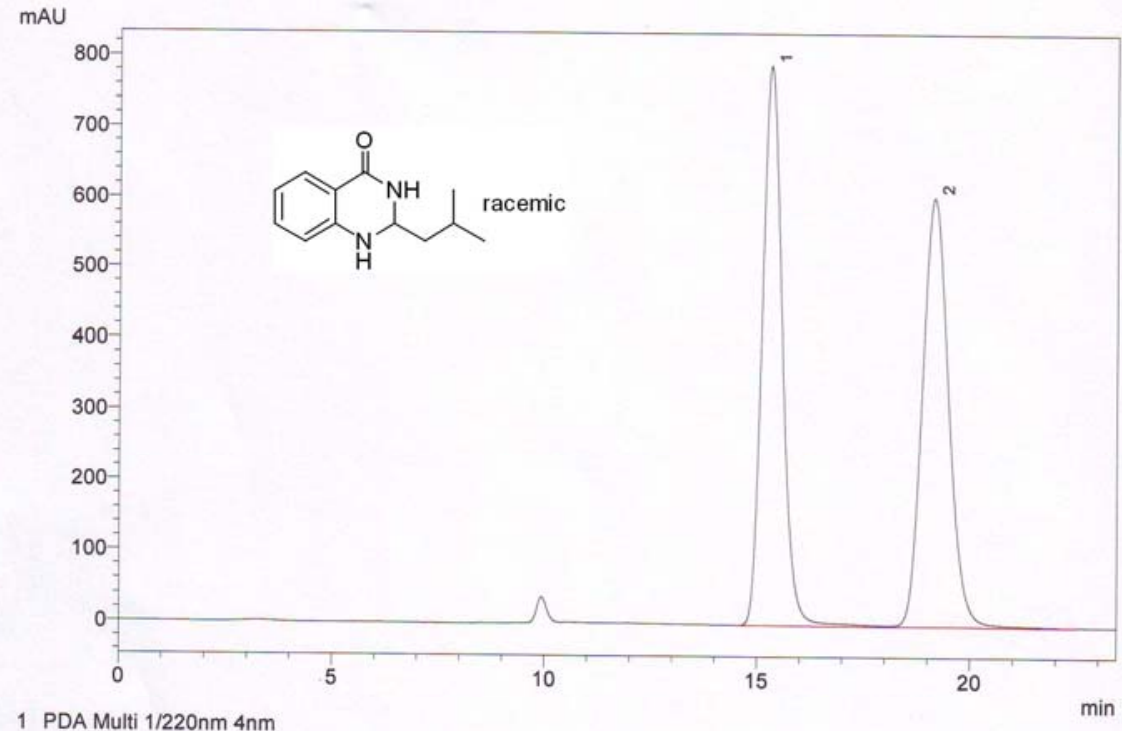

$\begin{array}{ccccr}\text { PDA Ch1 } 220 \mathrm{~nm} \text { 4nm } & & & \\ \text { Peak \# } & \text { Ret. Time } & \text { Area \% } & \text { Theoretical Plates } & k^{\prime} \\ 1 & 15.30 & 49.93 & 5170 & 0.00 \\ 2 & 19.14 & 50.07 & 4650 & 0.25 \\ \text { Total } & & 100.00 & & \end{array}$

mAU

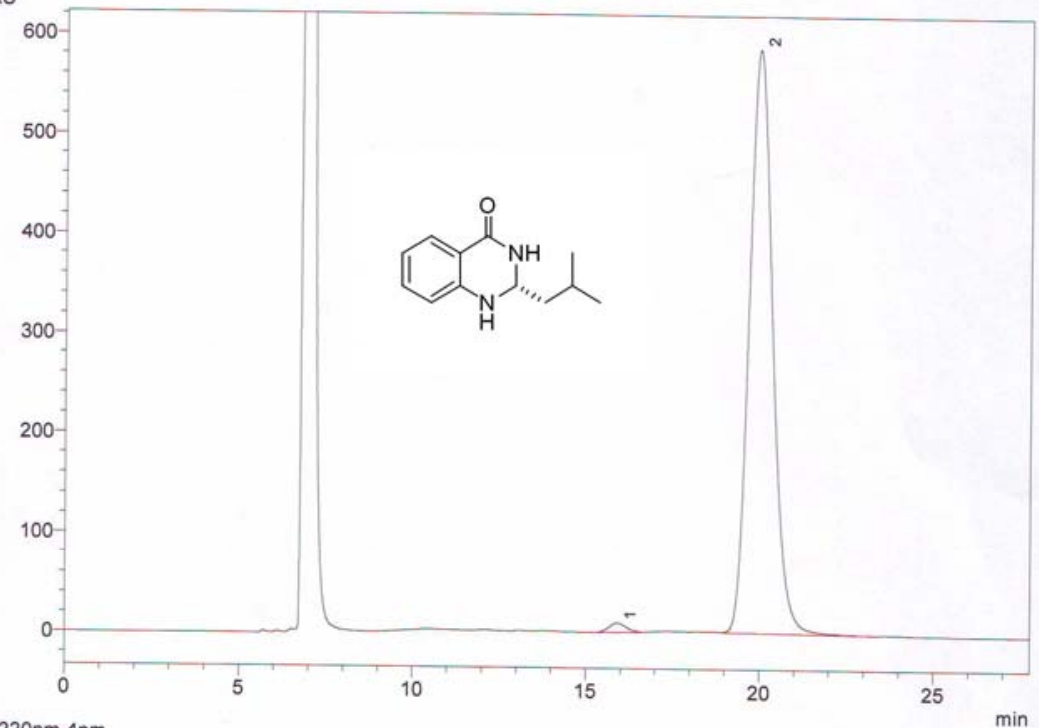

PDA Ch1 220nm 4nm

\begin{tabular}{|c|c|c|}
\hline Peak \# & Ret. Time & Area \% \\
\hline 1 & 15.90 & 1.12 \\
\hline 2 & 19.95 & 98.88 \\
\hline Total & & 100.00 \\
\hline
\end{tabular}


mAU

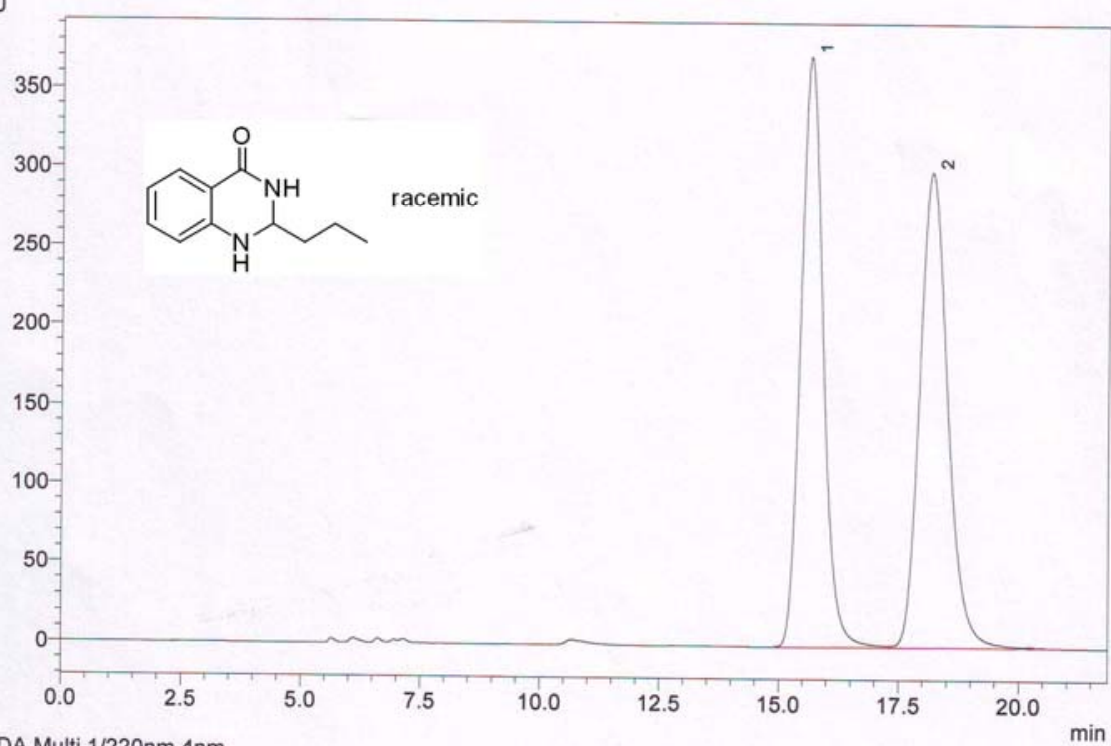

1 PDA Multi $1 / 220 \mathrm{~nm} 4 \mathrm{~nm}$

PDA Ch1 220nm 4nm

$\begin{array}{ccccr}\text { Peak \# } & \text { Ret. Time } & \text { Area \% } & \text { Theoretical Plates } & k^{\prime} \\ 1 & 15.64 & 49.81 & 5450 & 0.00 \\ 2 & 18.17 & 50.19 & 4739 & 0.16 \\ \text { Total } & & 100.00 & & \end{array}$

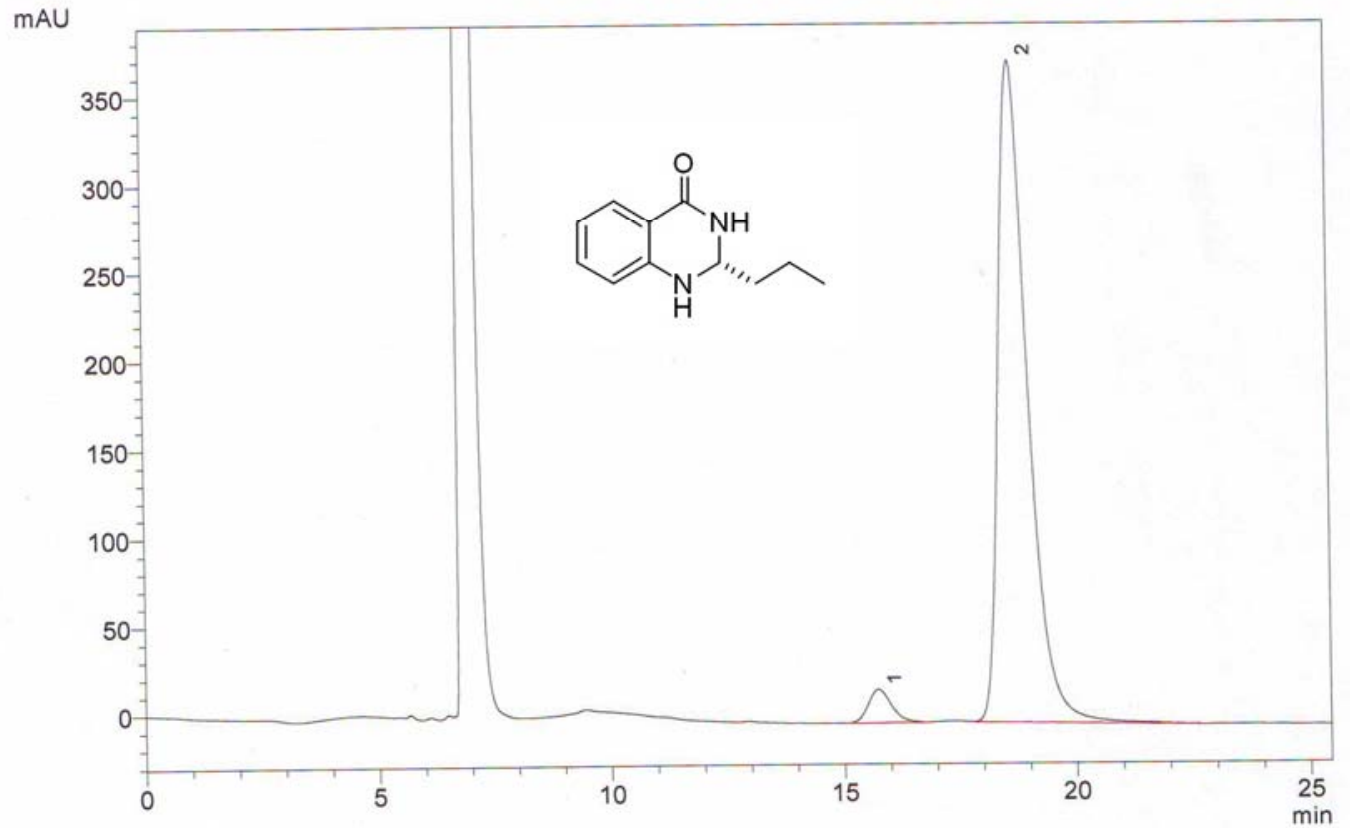

1 PDA Multi $1 / 220 \mathrm{~nm} 4 \mathrm{~nm}$

PDA Ch1 220nm 4nm

$\begin{array}{ccccr}\text { Peak \# } & \text { Ret. Time } & \text { Area \% } & \text { Theoretical Plates } & k^{\prime} \\ 1 & 15.73 & 3.82 & 4814 & 0.00 \\ 2 & 18.67 & 96.18 & 4459 & 0.19 \\ \text { Total } & & 100.00 & & \end{array}$


mAU

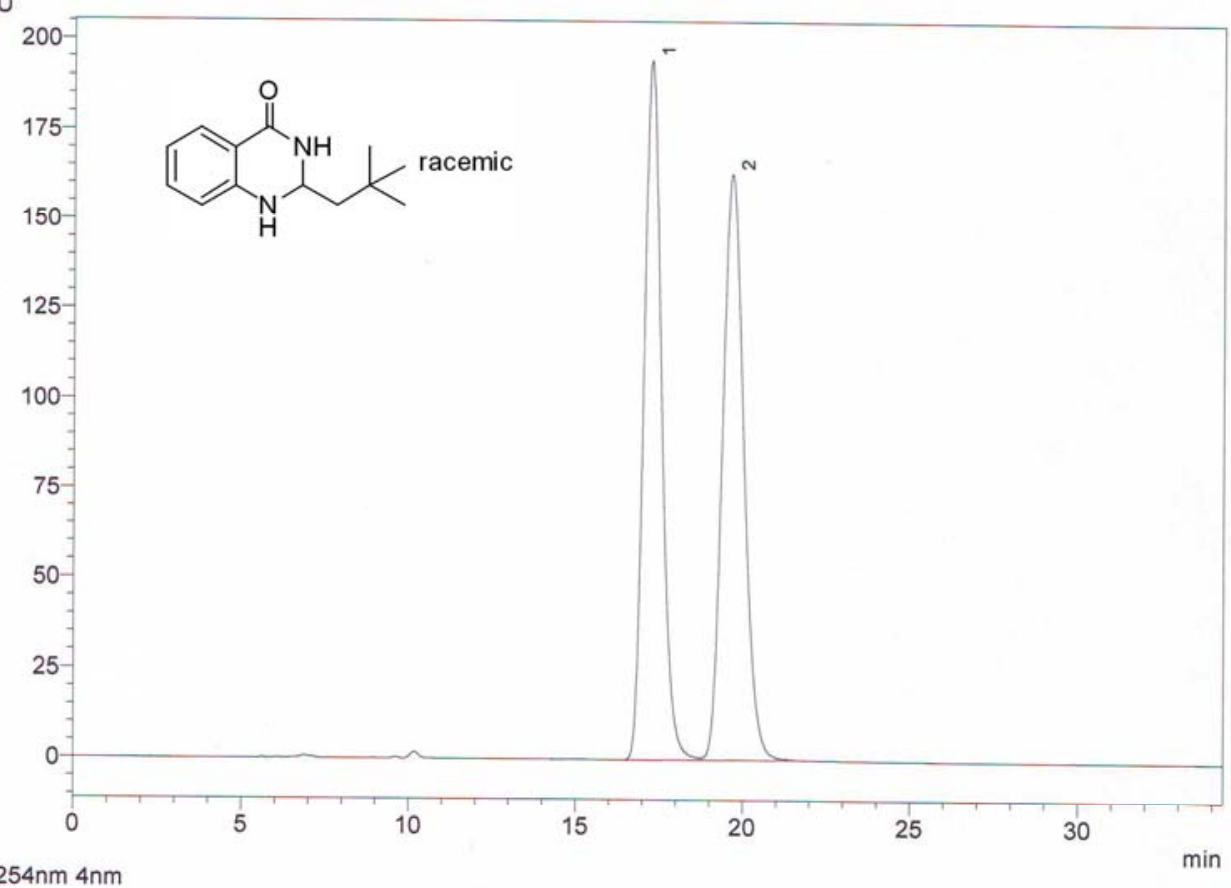

$1254 \mathrm{~nm} 4 \mathrm{~nm}$

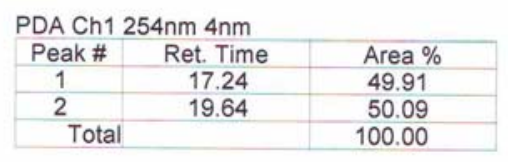

mAU

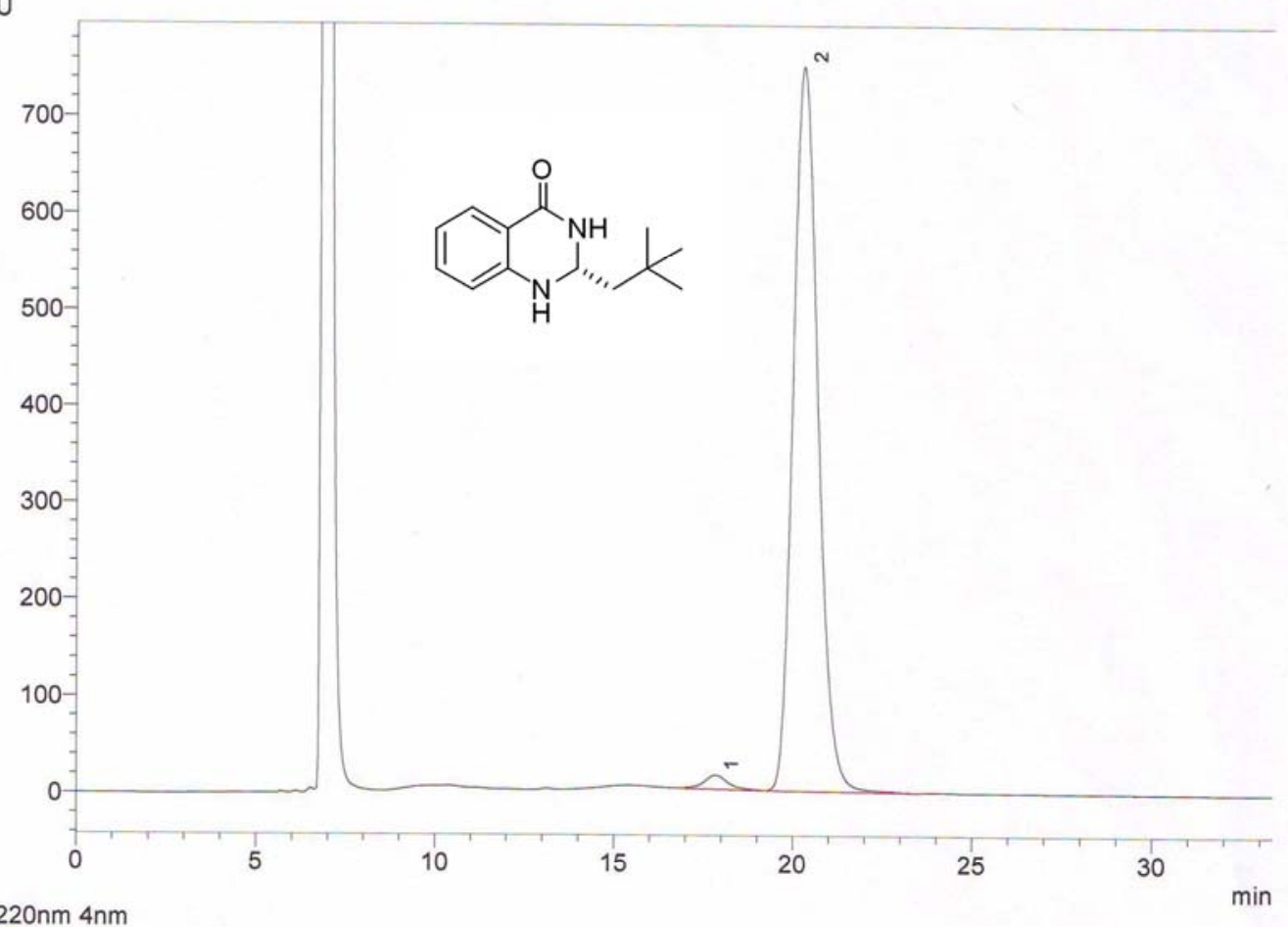

$1220 \mathrm{~nm} 4 \mathrm{~nm}$

$$
\begin{array}{ccc}
\text { PDA Ch1 } 220 \mathrm{~nm} \text { 4nm } & \\
\text { Peak \# } & \begin{array}{c}
\text { Ret. Time } \\
1
\end{array} & \text { Area \% } \\
2 & 17.83 & 1.76 \\
\text { Total } & 20.28 & 98.24 \\
\text { Tol } & & 100.00
\end{array}
$$

S-56 

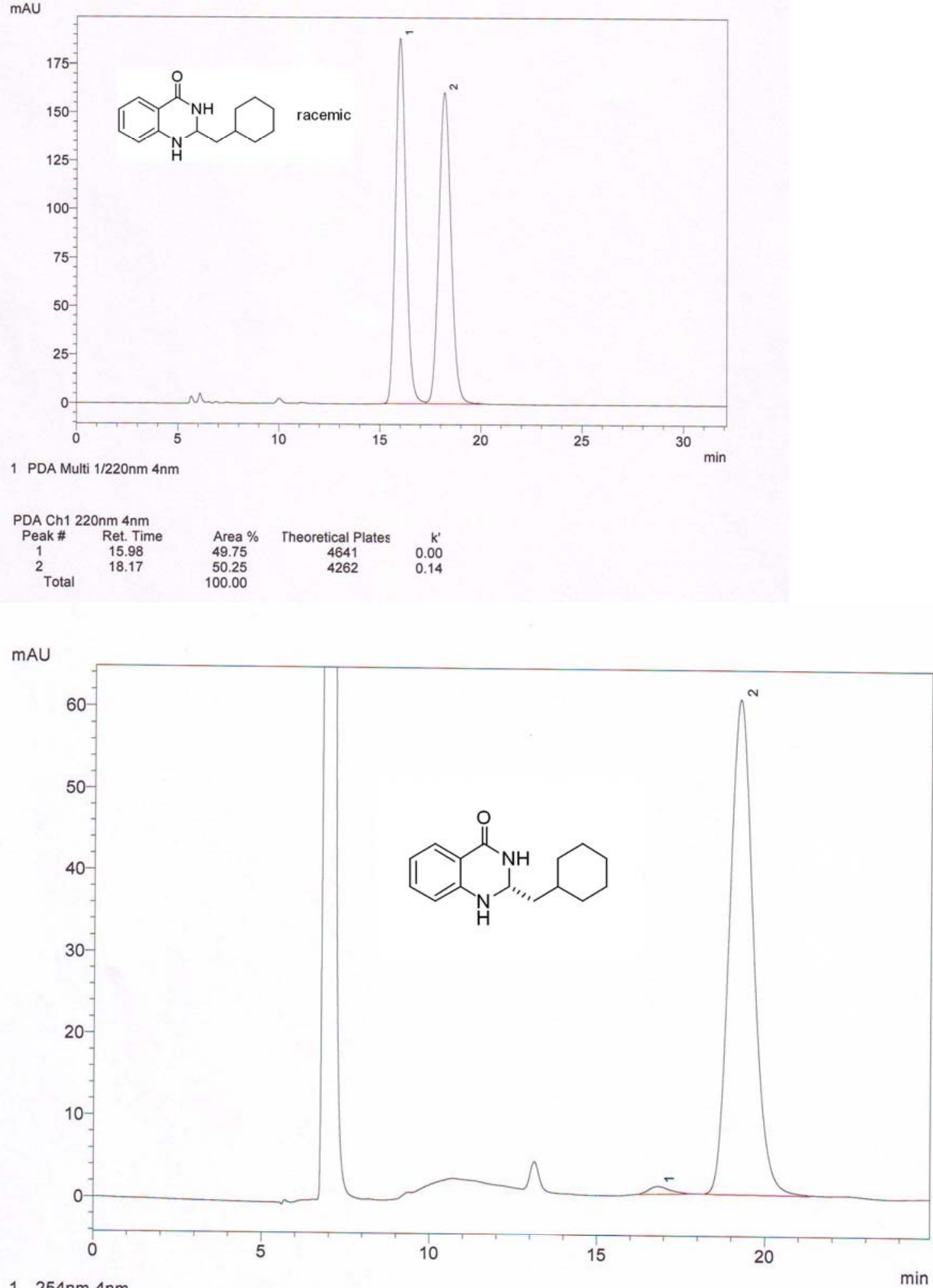

$1254 \mathrm{~nm} 4 \mathrm{~nm}$

PDA Ch1 254nm 4nm

\begin{tabular}{|c|c|c|}
\hline Peak \# & Ret. Time & Area \% \\
\hline 1 & 16.82 & 1.25 \\
\hline 2 & 19.21 & 98.75 \\
\hline Total & & 100.00 \\
\hline
\end{tabular}



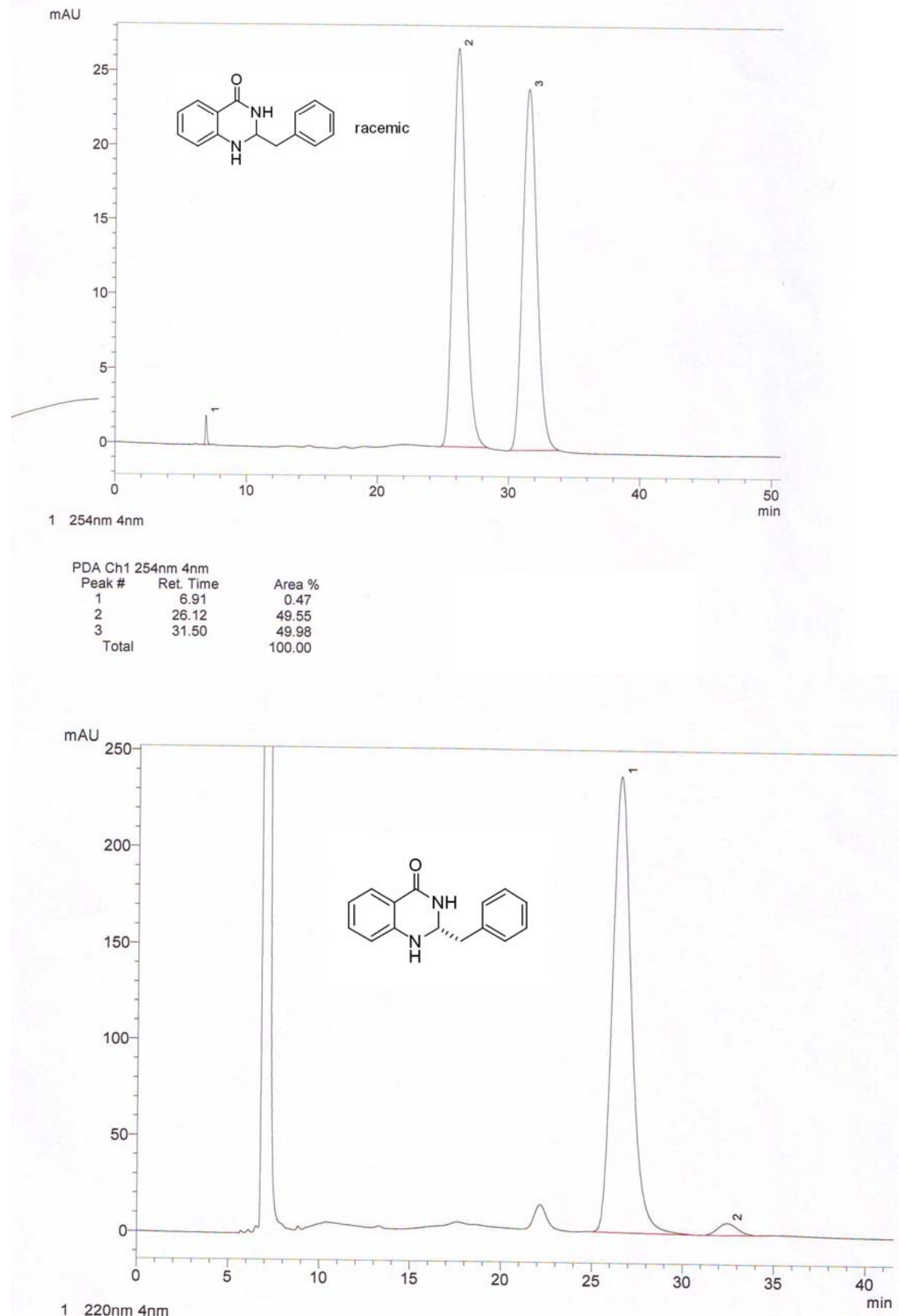

$\begin{array}{ccc}\text { PDA Ch1 220nm 4nm } & \\ \text { Peak \# } & \text { Ret. Time } & \text { Area \% } \\ 1 & 26.49 & 97.36 \\ 2 & 32.44 & 2.64 \\ \text { Total } & & 100.00\end{array}$




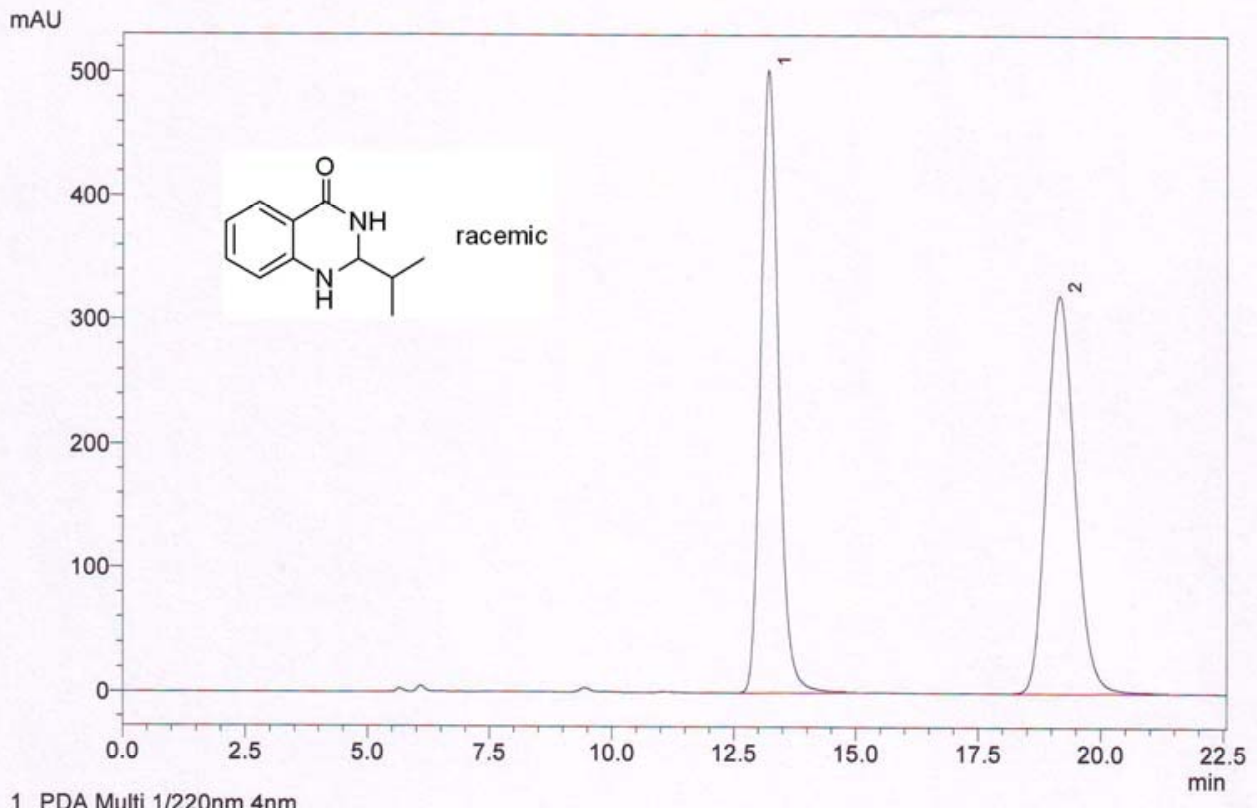

1 PDA Multi 1/220nm 4nm

\begin{tabular}{ccccr} 
PDA Ch1 & \multicolumn{2}{c}{ 220nm 4nm } \\
Peak \# & Ret. Time & Area \% & Theoretical Plates & K' $^{\prime}$ \\
1 & 13.20 & 49.91 & 6239 & 0.00 \\
2 & 19.15 & 50.09 & 5213 & 0.45 \\
Total & & 100.00 & &
\end{tabular}

mAU

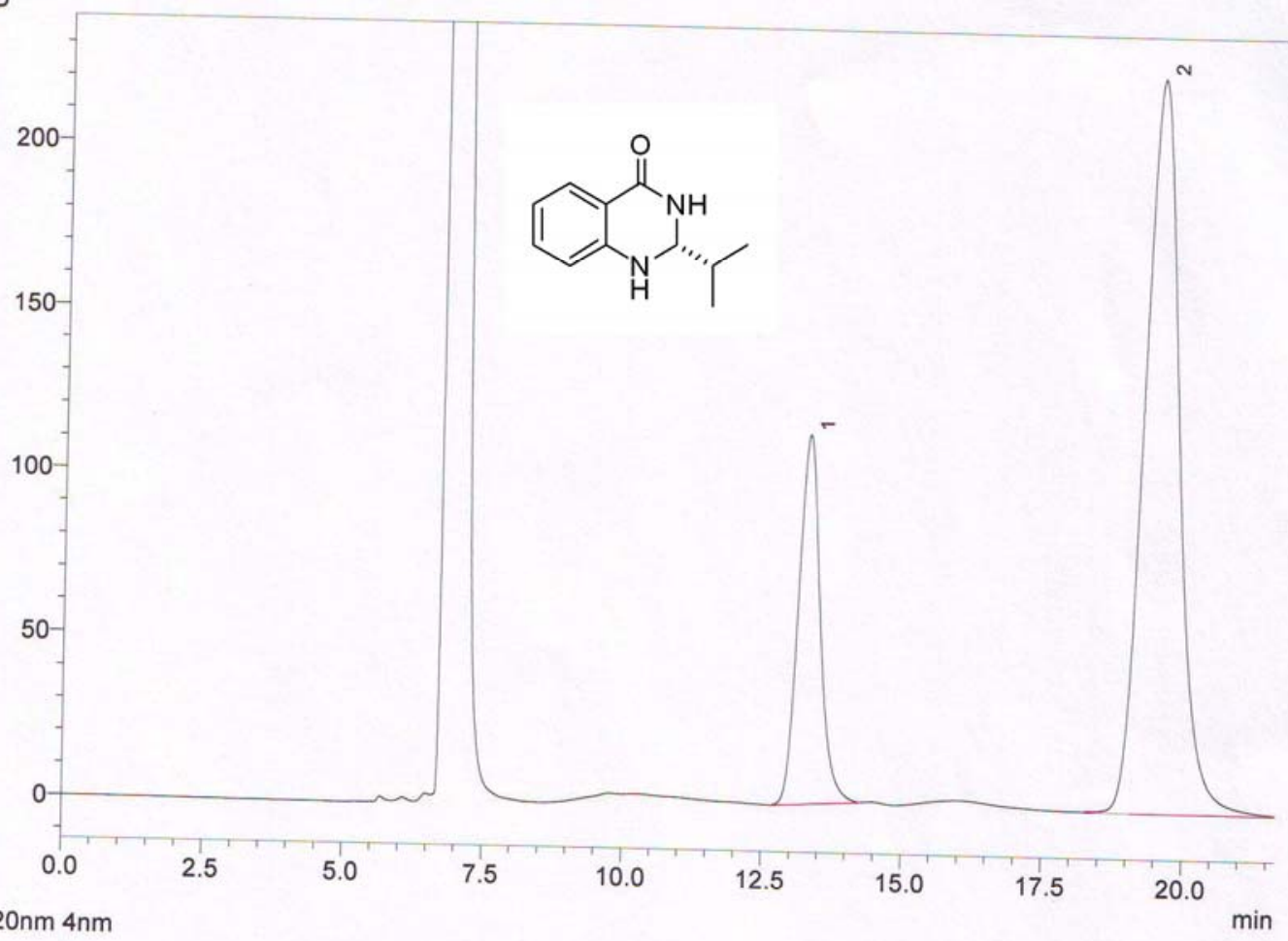

PDA Ch1 220nm 4nm

$\begin{array}{ccc}\text { Peak \# } & \text { Ret. Time } & \text { Area \% } \\ 1 & 13.28 & 23.94 \\ 2 & 19.50 & 76.06 \\ \text { Total } & & 100.00\end{array}$


mAU

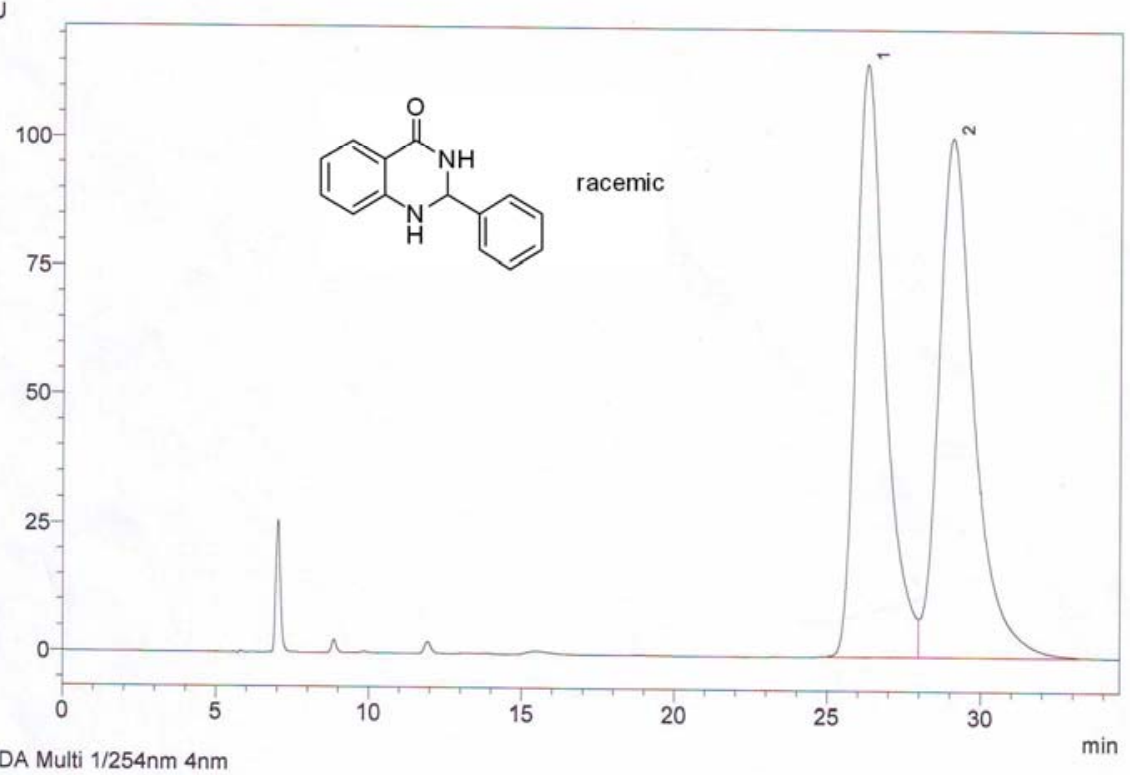

1 PDA Multi 1/254nm 4nm

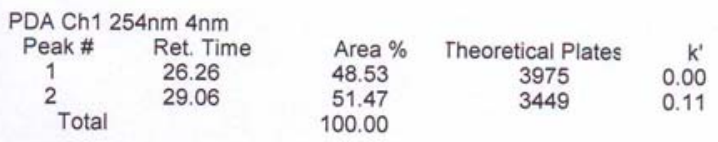

mAU

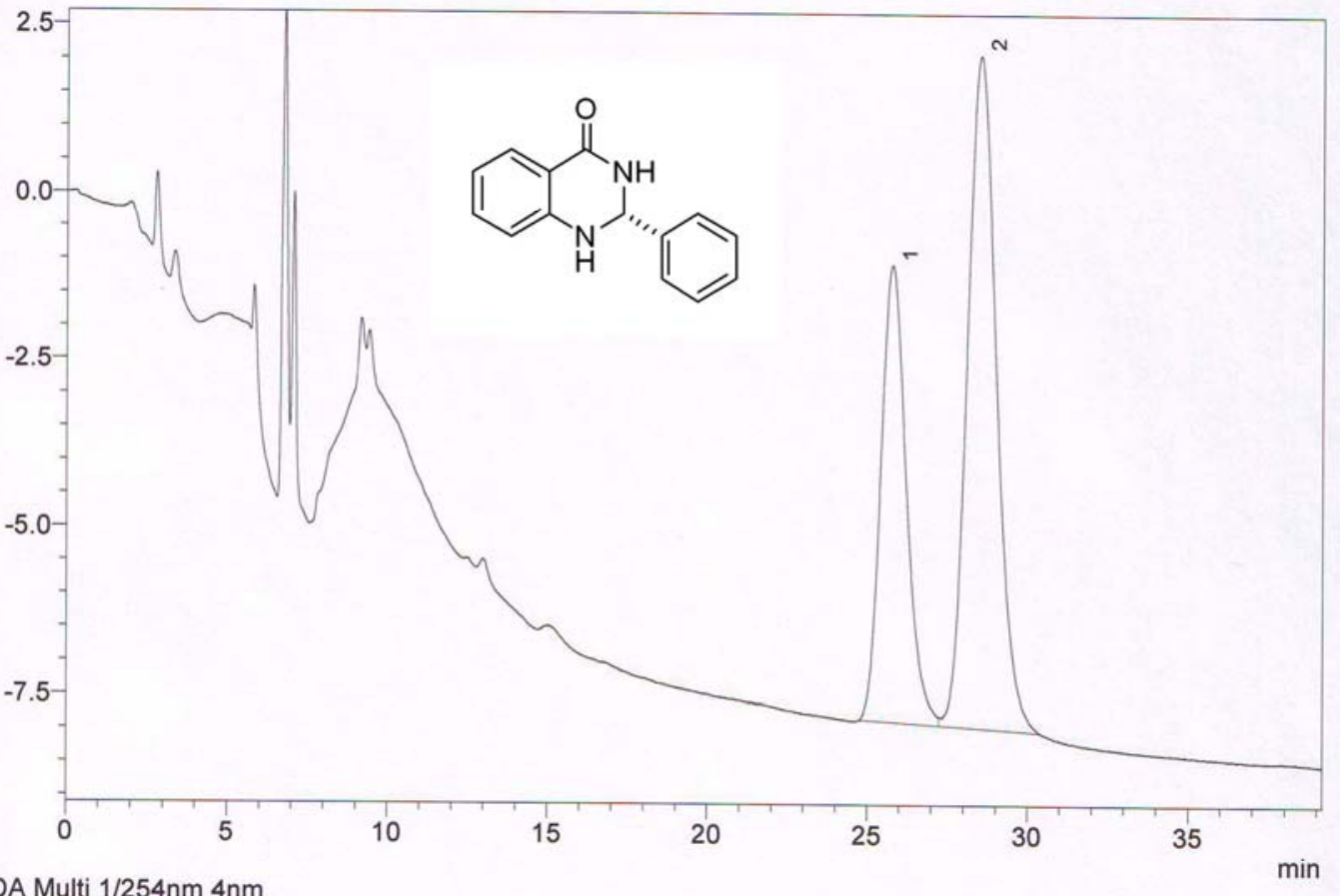

1 PDA Multi $1 / 254 \mathrm{~nm} 4 \mathrm{~nm}$

PDA Ch1 254nm 4nm

Peak \# Ret. Time

1

25.75

Area \%

Theoretical Plates

${ }^{2}$ Total

37.13

5135

$k^{\prime}$
0.00

100.00

S-60 

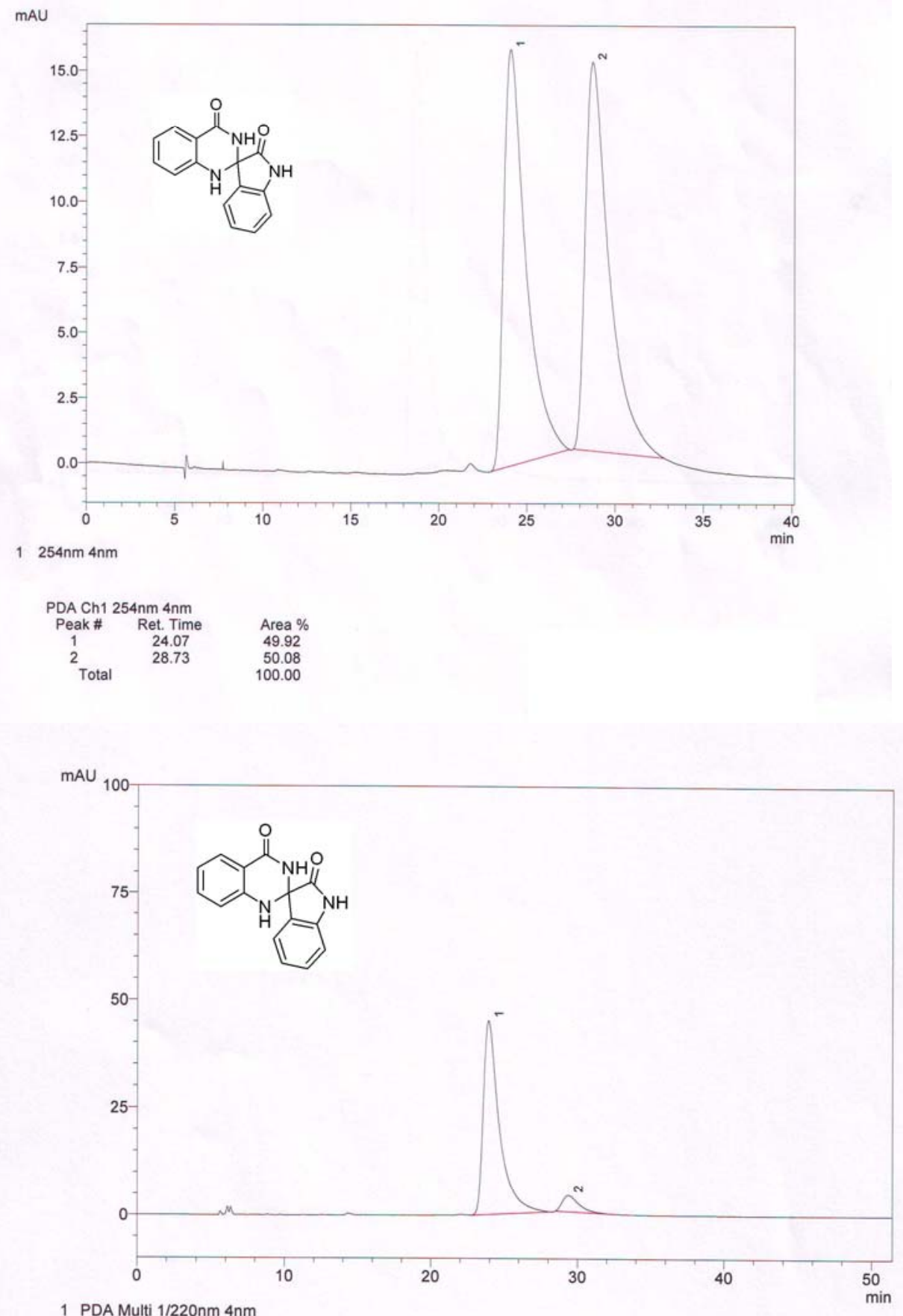

1 PDA Multi $1 / 220 \mathrm{~nm} 4 \mathrm{~nm}$

PDA Ch1 220nm 4nm

$\begin{array}{ccccr}\text { Peak \# } & \text { Ret. Time } & \text { Area \% } & \text { Theoretical Plates } & \mathrm{k}^{\prime} \\ 1 & 23.90 & 91.95 & 2808 & 0.00 \\ 2 & 29.35 & 8.05 & 3268 & 0.23 \\ \text { Total } & & 100.00 & & \end{array}$



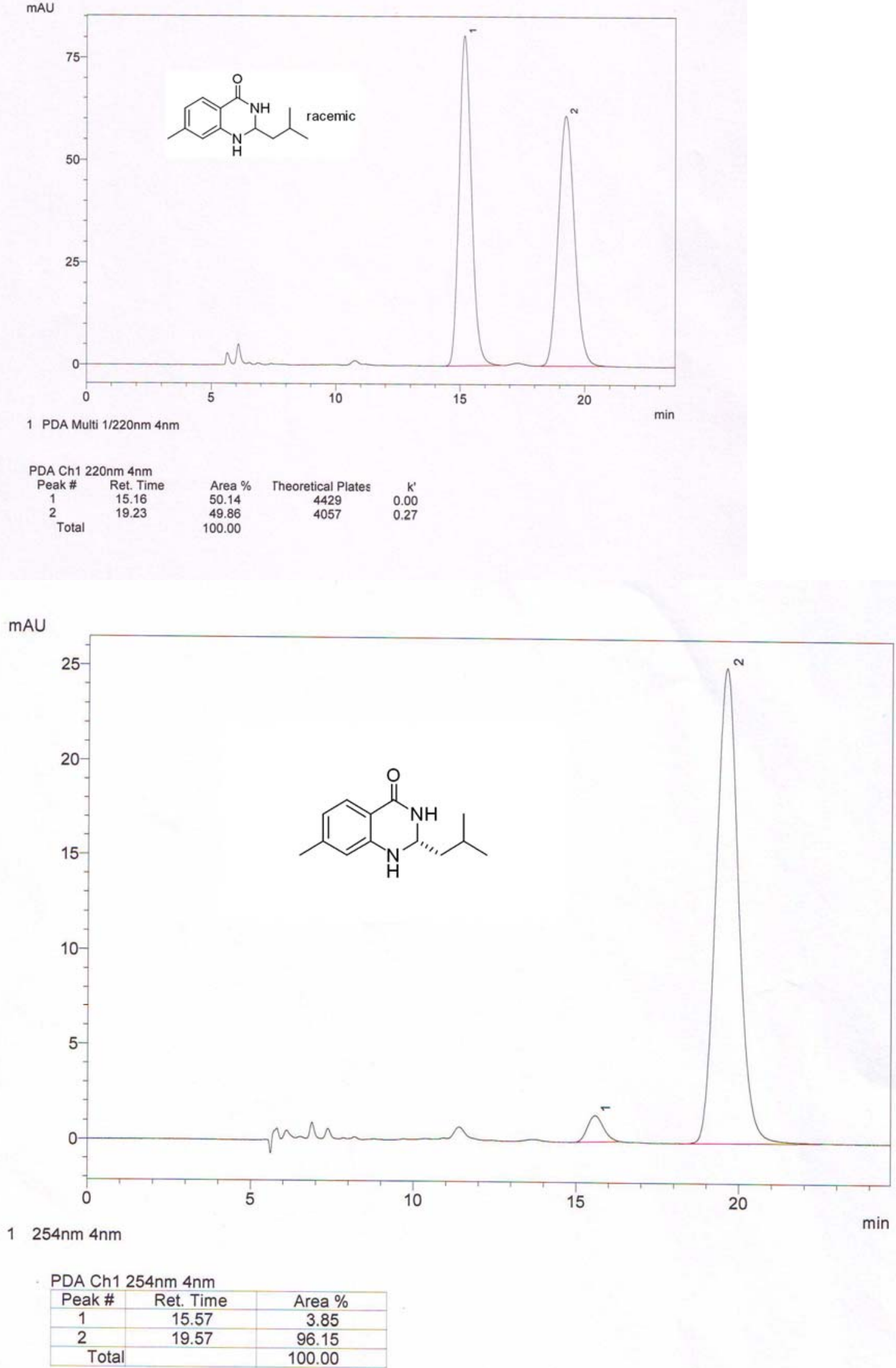


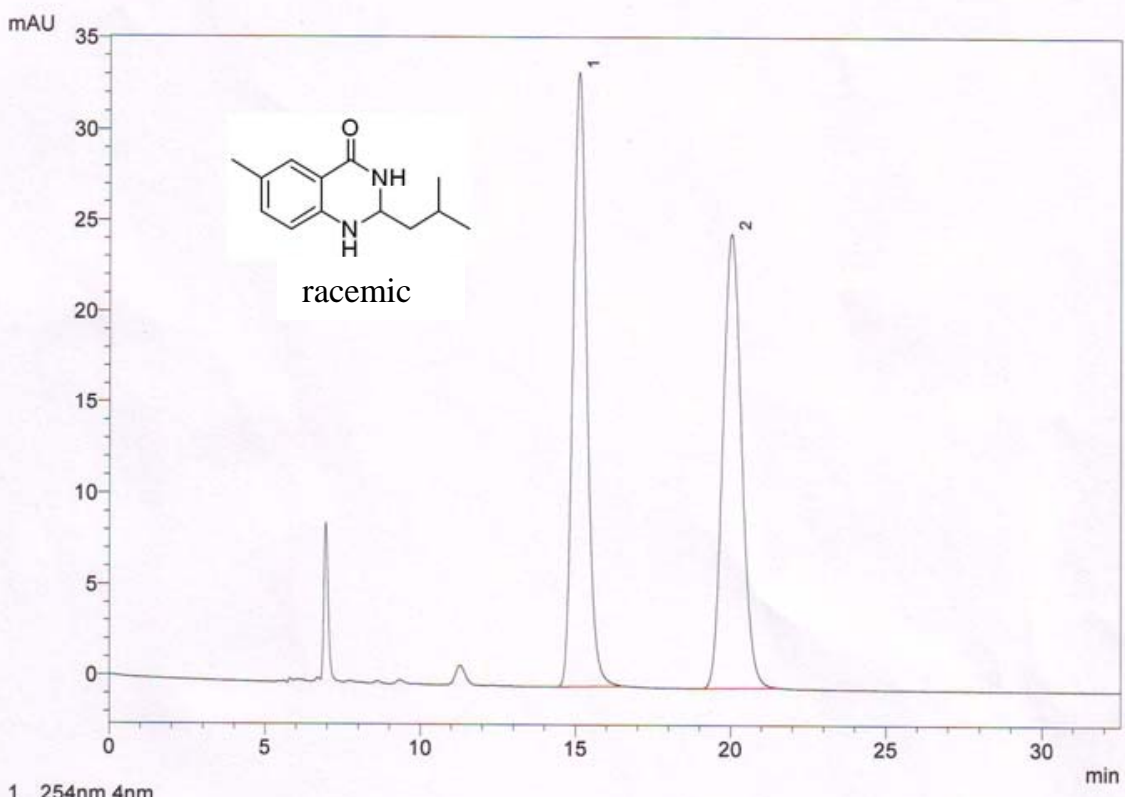

$1254 \mathrm{~nm} 4 \mathrm{~nm}$

\begin{tabular}{|c|c|c|}
\hline \multicolumn{3}{|c|}{ PDA Ch1 254nm 4nm } \\
\hline Peak \# & Ret. Time & Area \% \\
\hline 2 & 20.02 & 49.95 \\
\hline Tota & & 100.00 \\
\hline
\end{tabular}

MAU

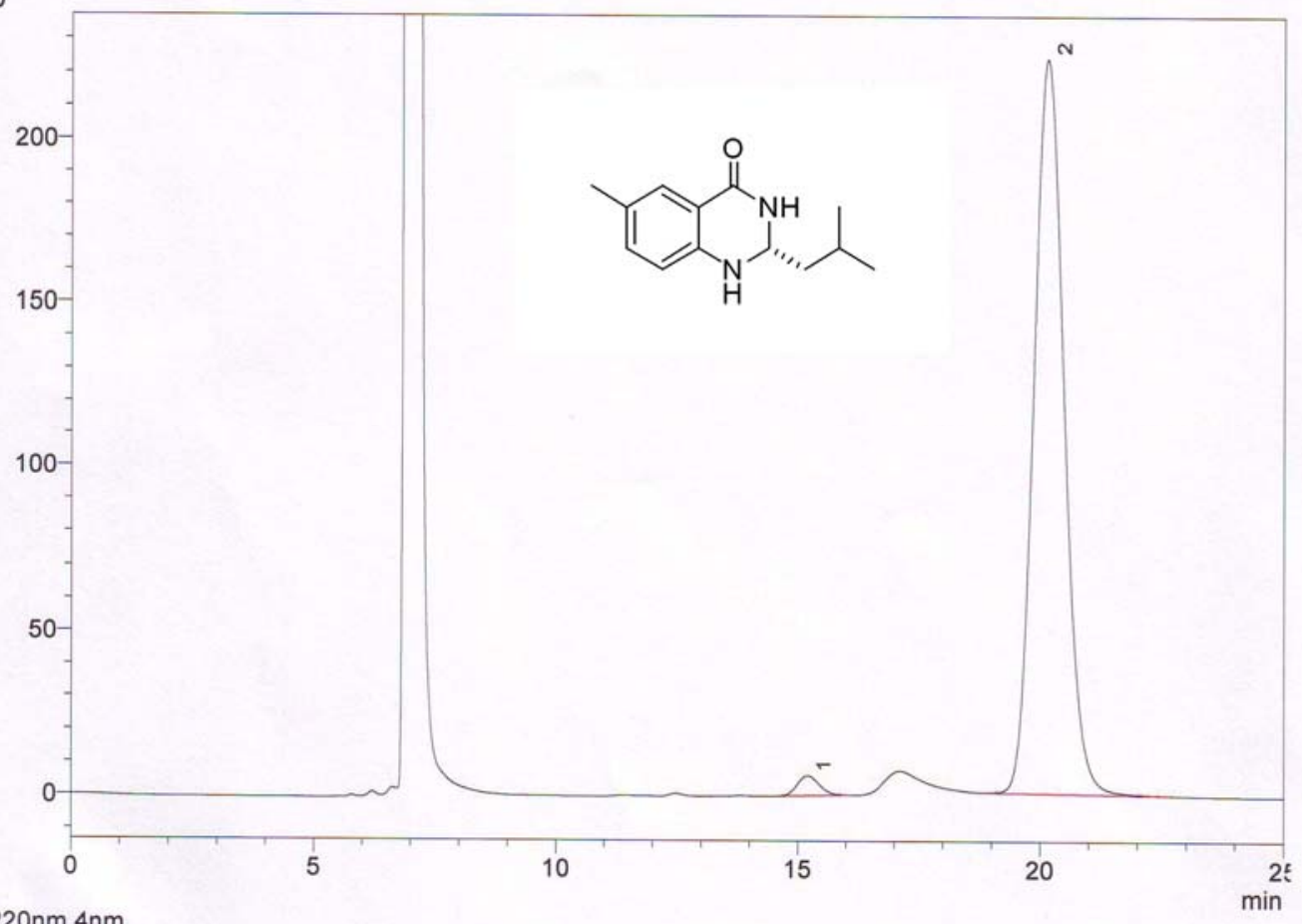

$1220 \mathrm{~nm} 4 \mathrm{~nm}$

$\begin{array}{ccc}\text { PDA Ch1 } & 220 \mathrm{~nm} \text { 4nm } & \\ \text { Peak \# } & \text { Ret. Time } & \text { Area \% } \\ 1 & 15.20 & 1.96 \\ 2 & 20.14 & 98.04 \\ \text { Total } & & 100.00\end{array}$




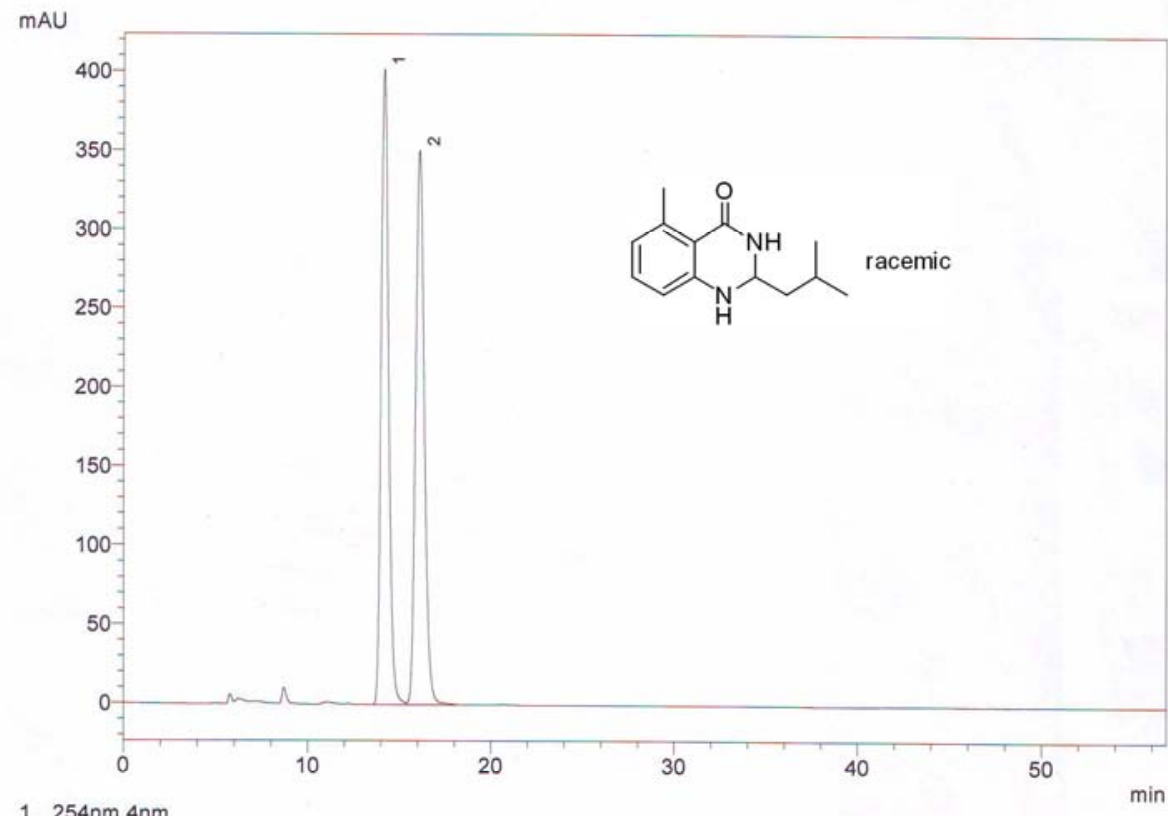

$1254 \mathrm{~nm} 4 \mathrm{~nm}$

\begin{tabular}{l} 
PDA Ch1 $254 \mathrm{~nm}$ 4nm \\
\begin{tabular}{|c|c|c|}
\hline Peak \# & Ret. Time & Area $\%$ \\
\hline 1 & 14.17 & 49.85 \\
\hline 2 & 16.08 & 50.15 \\
\hline Total & & 100.00 \\
\hline
\end{tabular} \\
\hline
\end{tabular}

mAU

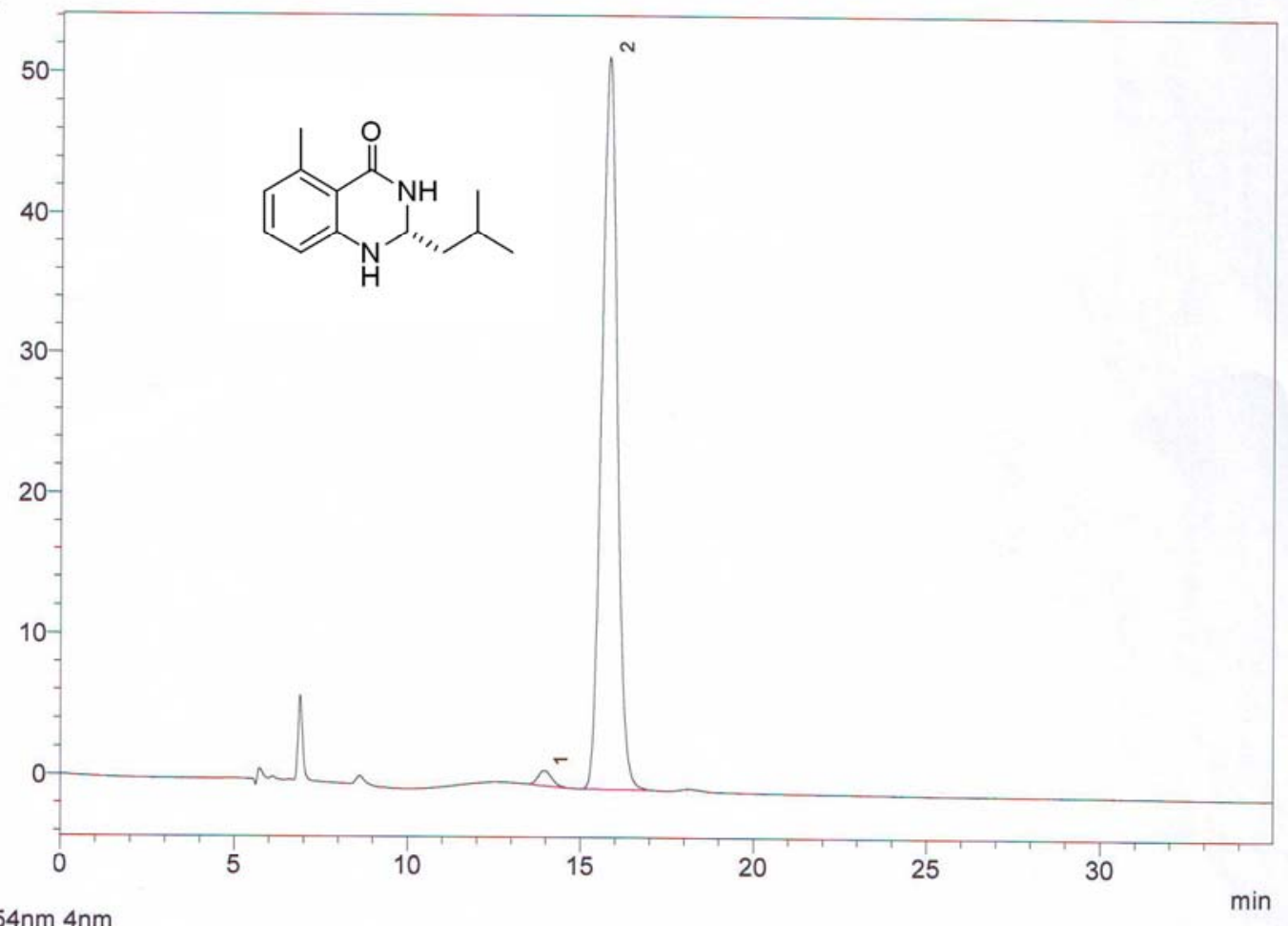

$1254 \mathrm{~nm} 4 \mathrm{~nm}$

PDA Ch1 254nm 4nm

\begin{tabular}{|c|c|c|}
\hline Peak \# & Ret. Time & Area \% \\
\hline 1 & 13.97 & 1.76 \\
\hline 2 & 15.78 & 98.24 \\
\hline Total & & 100.00 \\
\hline
\end{tabular}


TAU

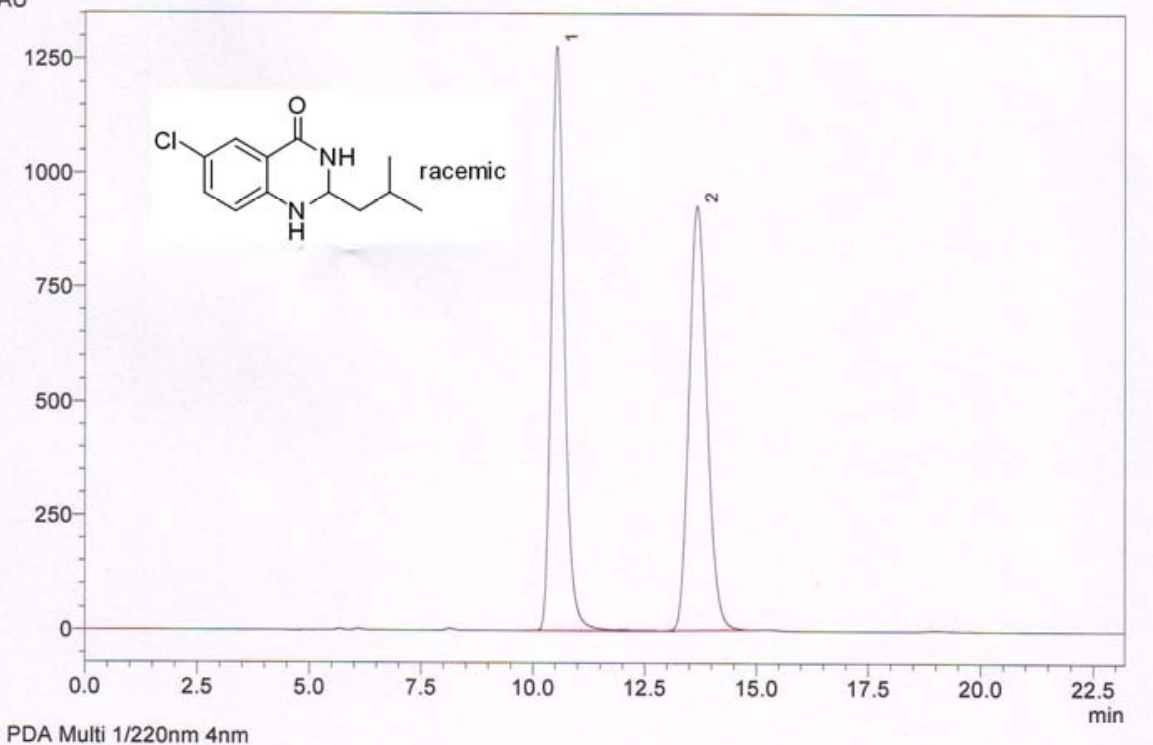

1 PDA Multi $1 / 220 \mathrm{~nm} 4 \mathrm{~nm}$

$\begin{array}{ccccr}\text { PDA Ch } 220 \mathrm{~nm} \text { nm } & & & \\ \text { Peak \# } & \text { Ret. Time } & \text { Area \% } & \text { Theoretical Plates } & \mathbf{k}^{\prime} \\ 1 & 10.53 & 50.00 & 6341 & 0.00 \\ 2 & 13.67 & 50.00 & 5430 & 0.30 \\ \text { Total } & & 100.00 & & \end{array}$

TAU

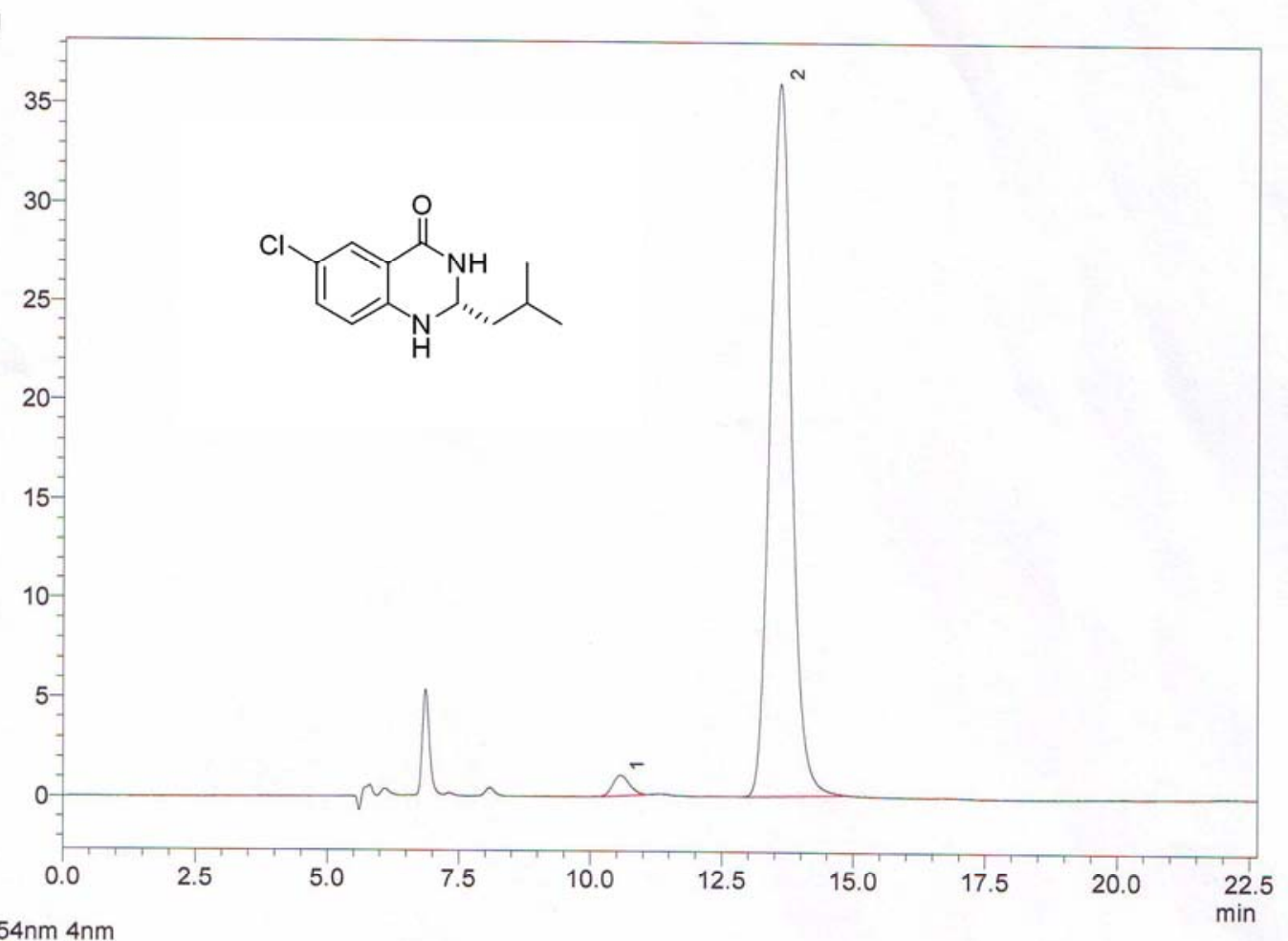

$1254 \mathrm{~nm} 4 \mathrm{~nm}$

PDA Ch 254nm 4nm

Peak \# Ret Time

\begin{tabular}{|c|c|c|}
\hline Peak \# & Ret. Time & Area \% \\
\hline 1 & 10.58 & 2.19 \\
\hline 2 & 13.57 & 97.81 \\
\hline Total & & 100.00 \\
\hline
\end{tabular}

S-65 

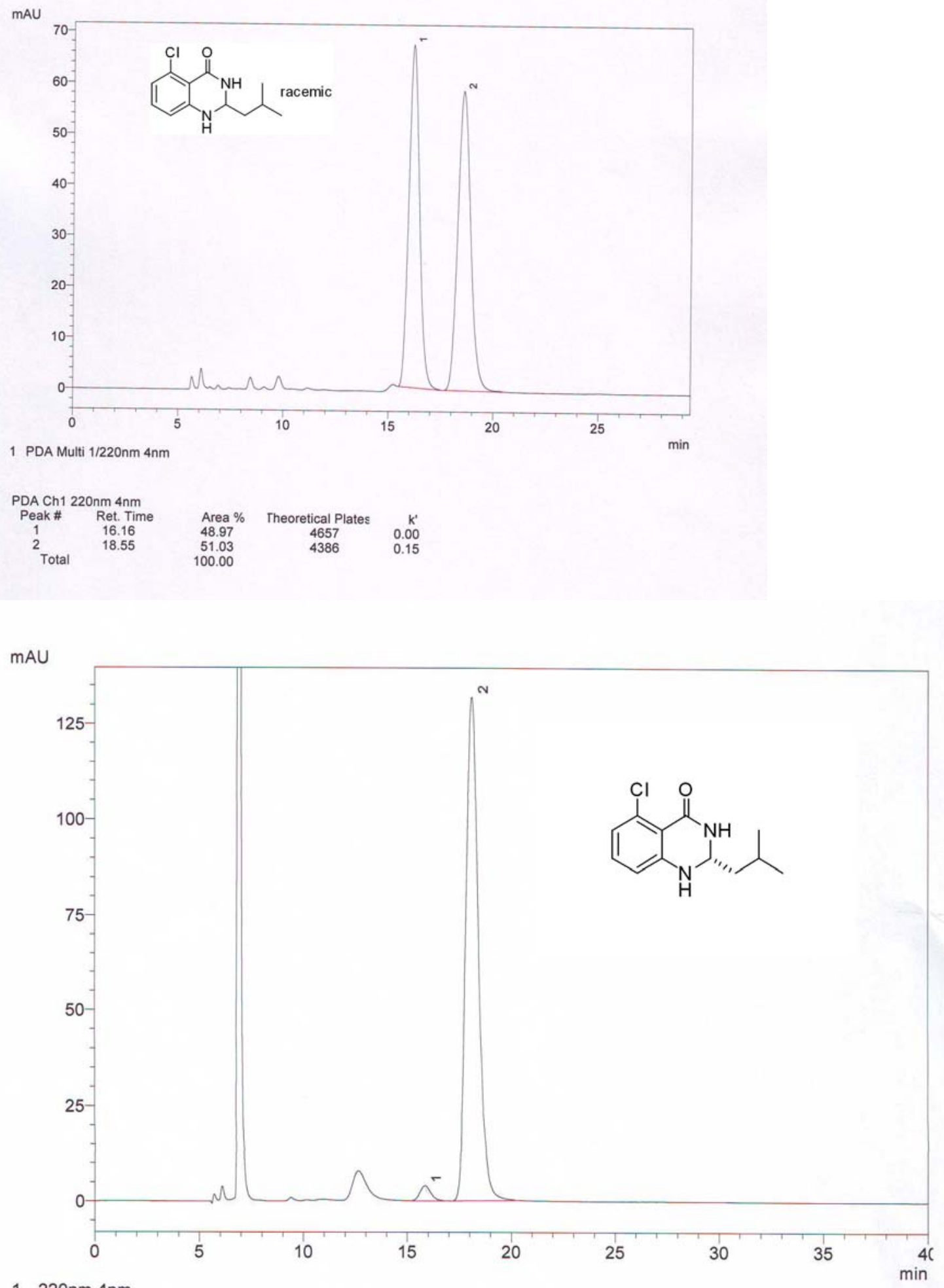

$1220 \mathrm{~nm} 4 \mathrm{~nm}$

$\begin{array}{ccc}\text { PDA Ch1 } 220 \mathrm{~nm} \text { 4nm } & \\ \text { Peak \# } & \text { Ret. Time } & \text { Area \% } \\ 1 & 15.85 & 2.54 \\ 2 & 18.10 & 97.46 \\ \text { Total } & & 100.00\end{array}$




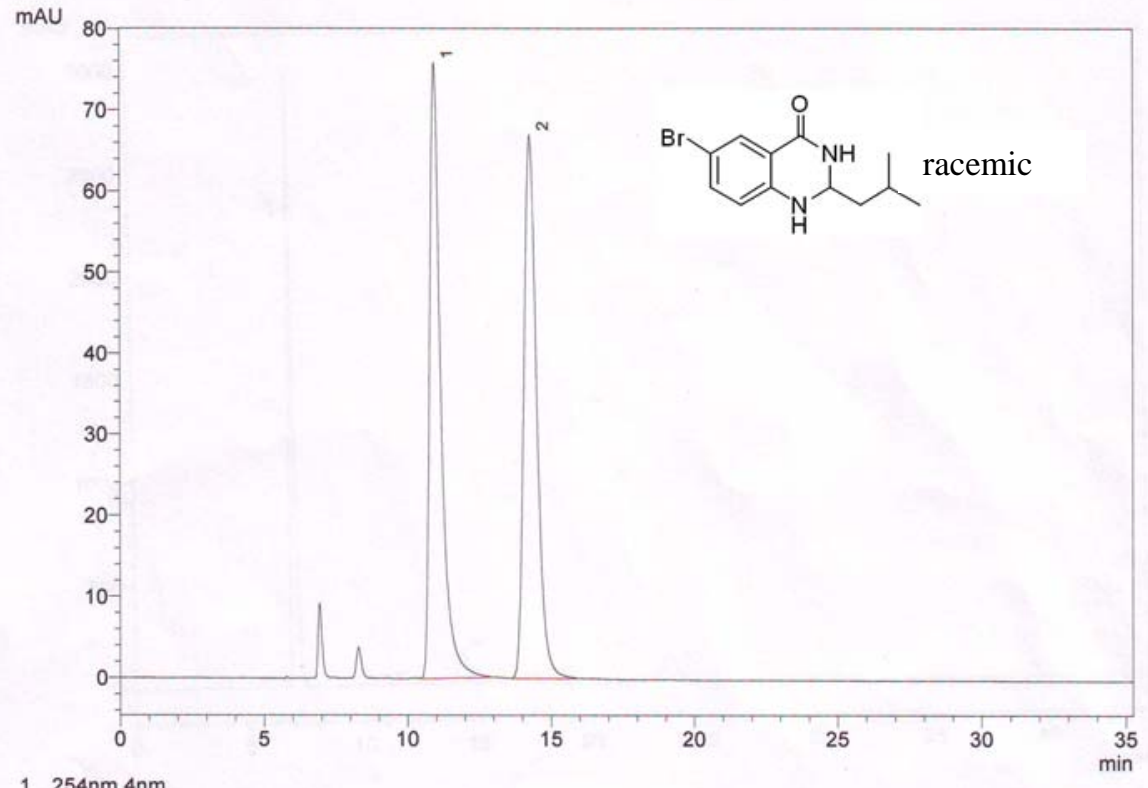

$1254 \mathrm{~nm} 4 \mathrm{~nm}$
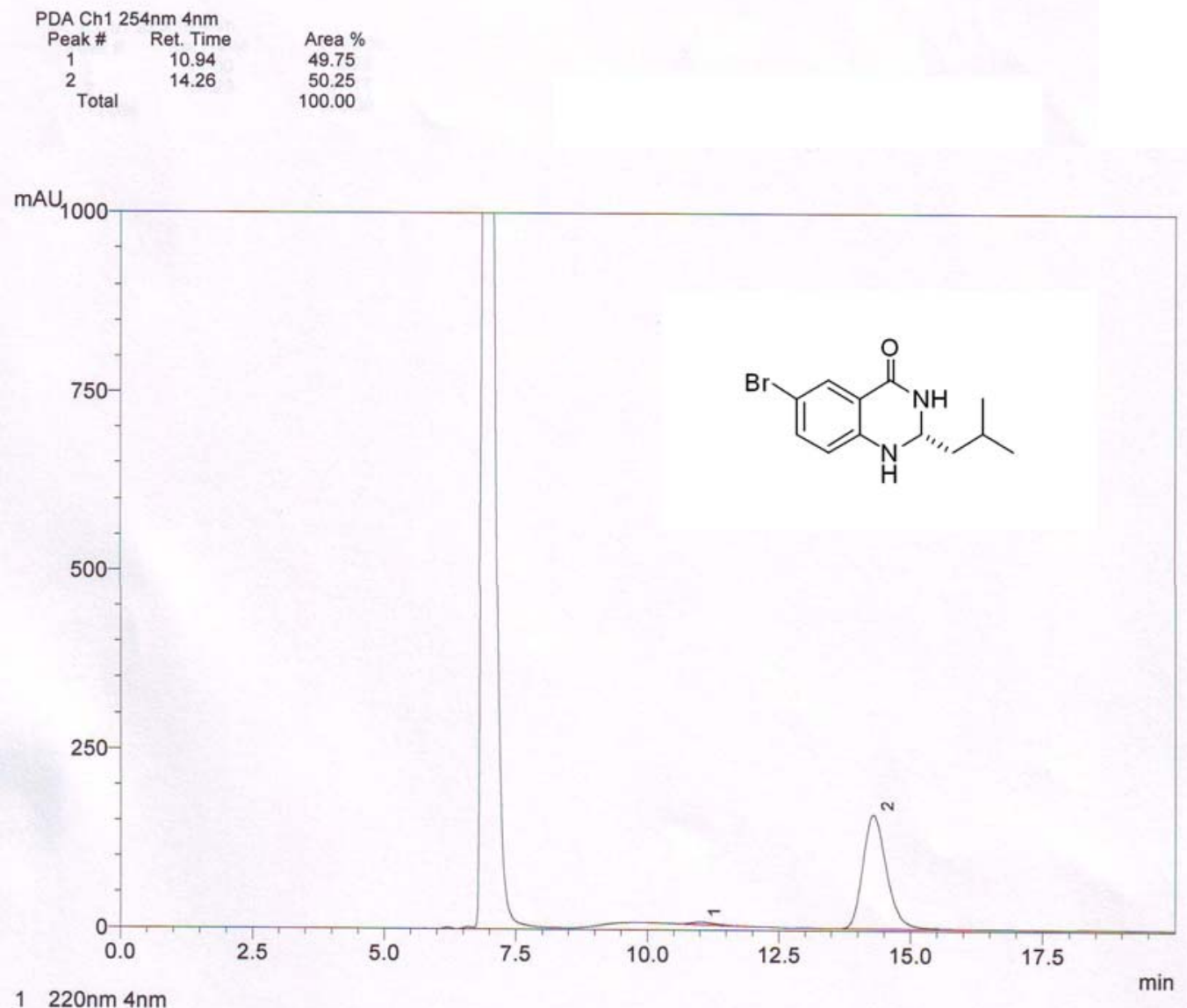

$1220 \mathrm{~nm} 4 \mathrm{~nm}$

PDA Ch1 220nm 4nm

$\begin{array}{ccc}\text { Peak \# } & \text { Ret. Time } & \text { Area \% } \\ 1 & 11.00 & 2.23 \\ 2 & 14.29 & 97.77 \\ \text { Total } & & 100.00\end{array}$




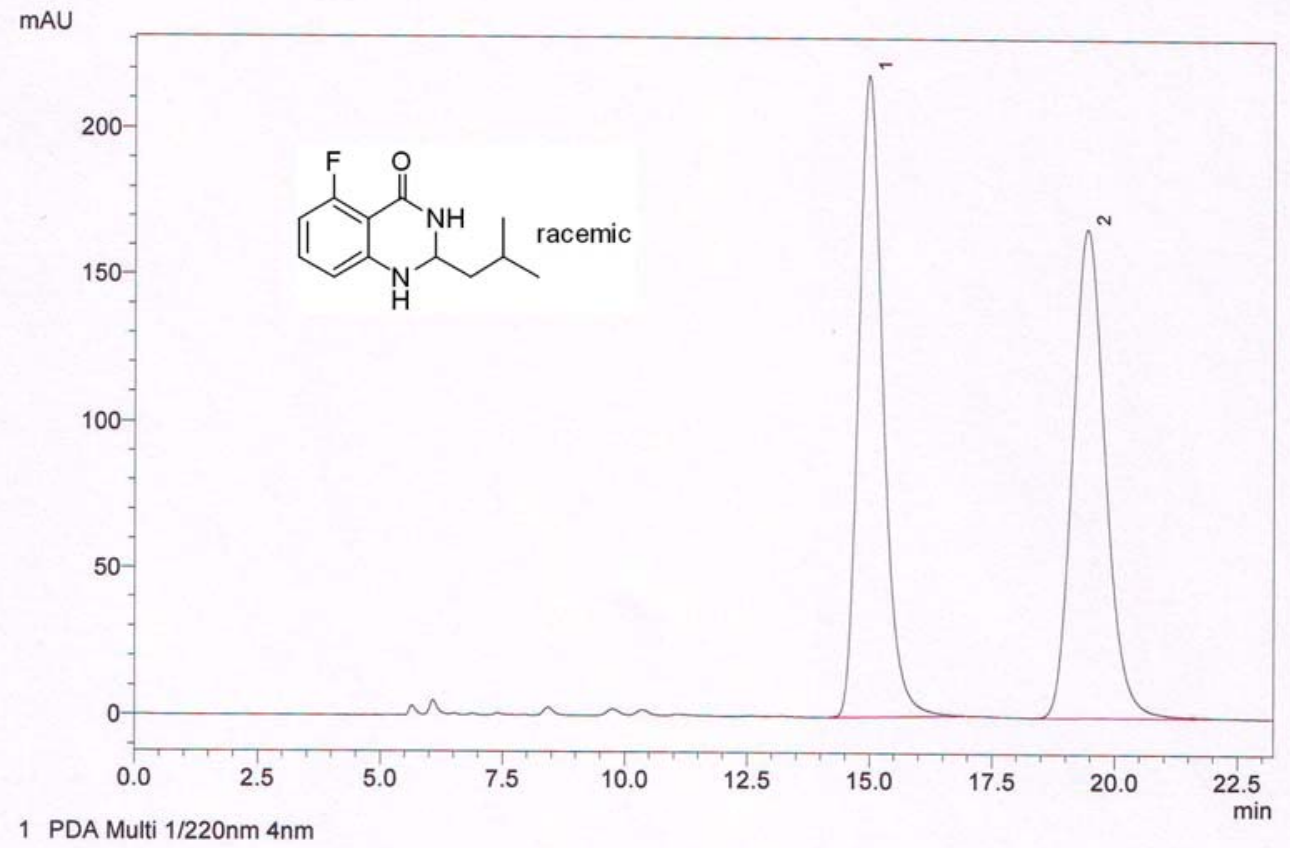

$\begin{array}{ccccr}\text { PDA Ch1 } 220 \mathrm{~nm} \text { 4nm } & & & \\ \text { Peak \# } & \text { Ret. Time } & \text { Area \% } & \text { Theoretical Plates } & \text { K' }^{\prime} \\ 1 & 14.97 & 49.90 & 4368 & 0.00 \\ 2 & 19.43 & 50.10 & 4162 & 0.30 \\ \text { Total } & & 100.00 & & \end{array}$

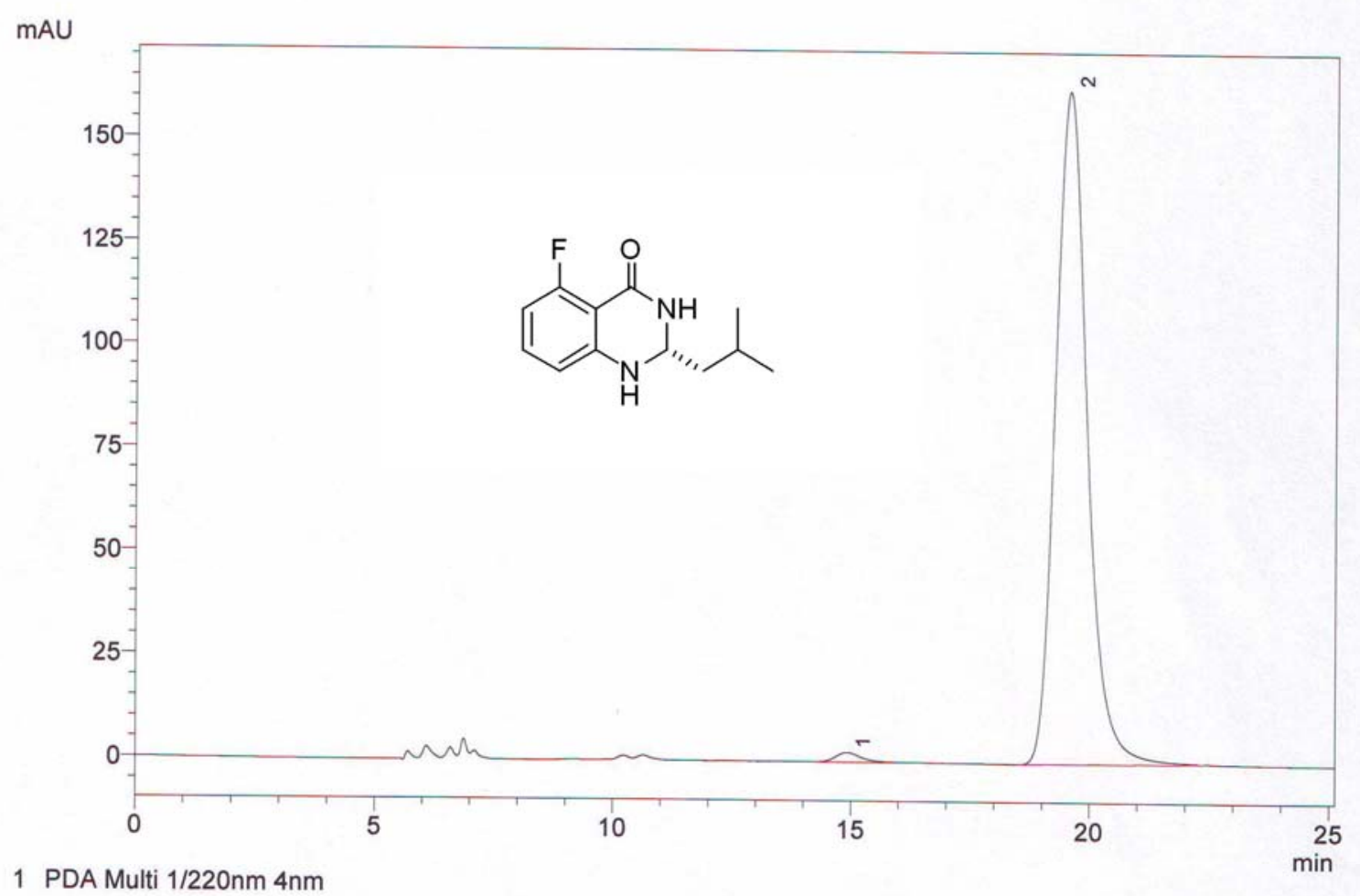

PDA Ch1 220nm 4nm

$\begin{array}{ccccr}\text { Peak \# } & \text { Ret. Time } & \text { Area \% } & \text { Theoretical Plates } & \text { k' }^{\prime} \\ 1 & 14.91 & 1.04 & 4124 & 0.00 \\ 2 & 19.54 & 98.96 & 4316 & 0.31 \\ \text { Total } & & 100.00 & & \end{array}$



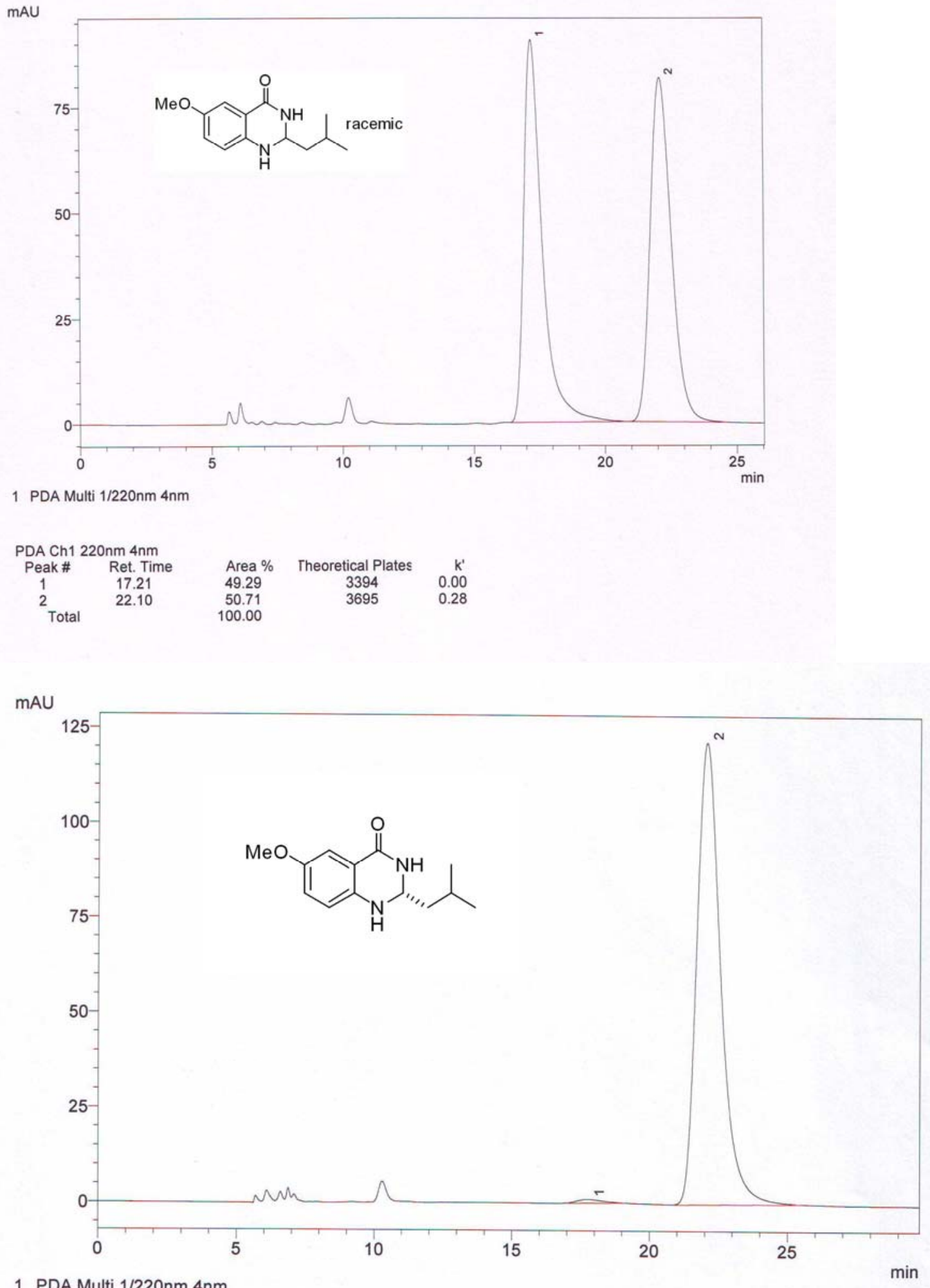

$\begin{array}{ccccr}\text { PDA Ch1 } 220 \mathrm{~nm} \text { 4nm } & & & \\ \text { Peak \# } & \text { Ret. Time } & \text { Area \% } & \text { Theoretical Plates } & \mathbf{k}^{\prime} \\ 1 & 17.77 & 0.81 & 1717 & 0.00 \\ 2 & 22.04 & 99.19 & 3302 & 0.24 \\ \text { Total } & & 100.00 & & \end{array}$


mAU

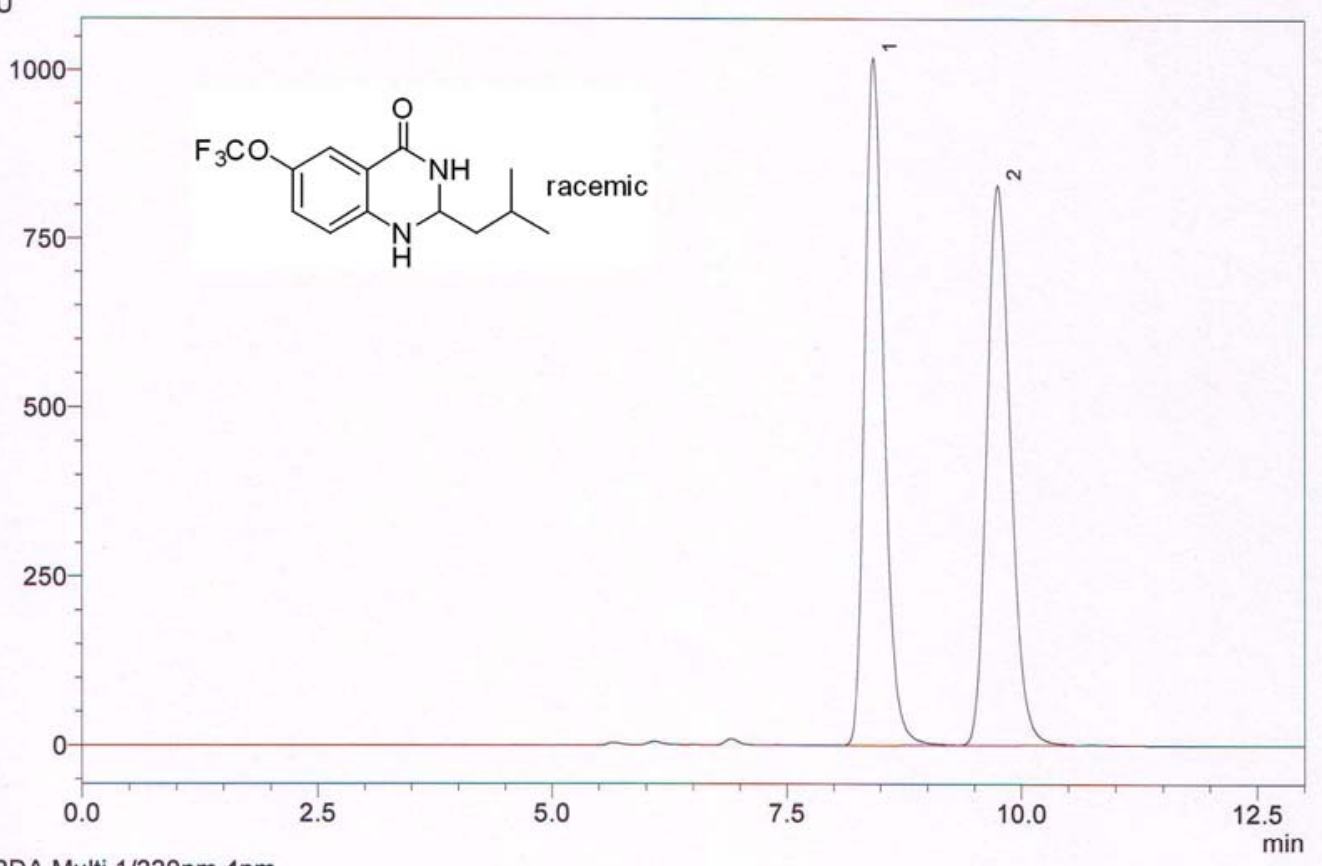

1 PDA Multi 1/220nm 4nm

PDA Ch1 220nm 4nm

Peak \# Ret. Time

1

Total

8.42

Area \%

Theoretical Plates

7899

50.04

6985

$$
\begin{array}{r}
k^{\prime} \\
0.00 \\
0.16
\end{array}
$$

mAU

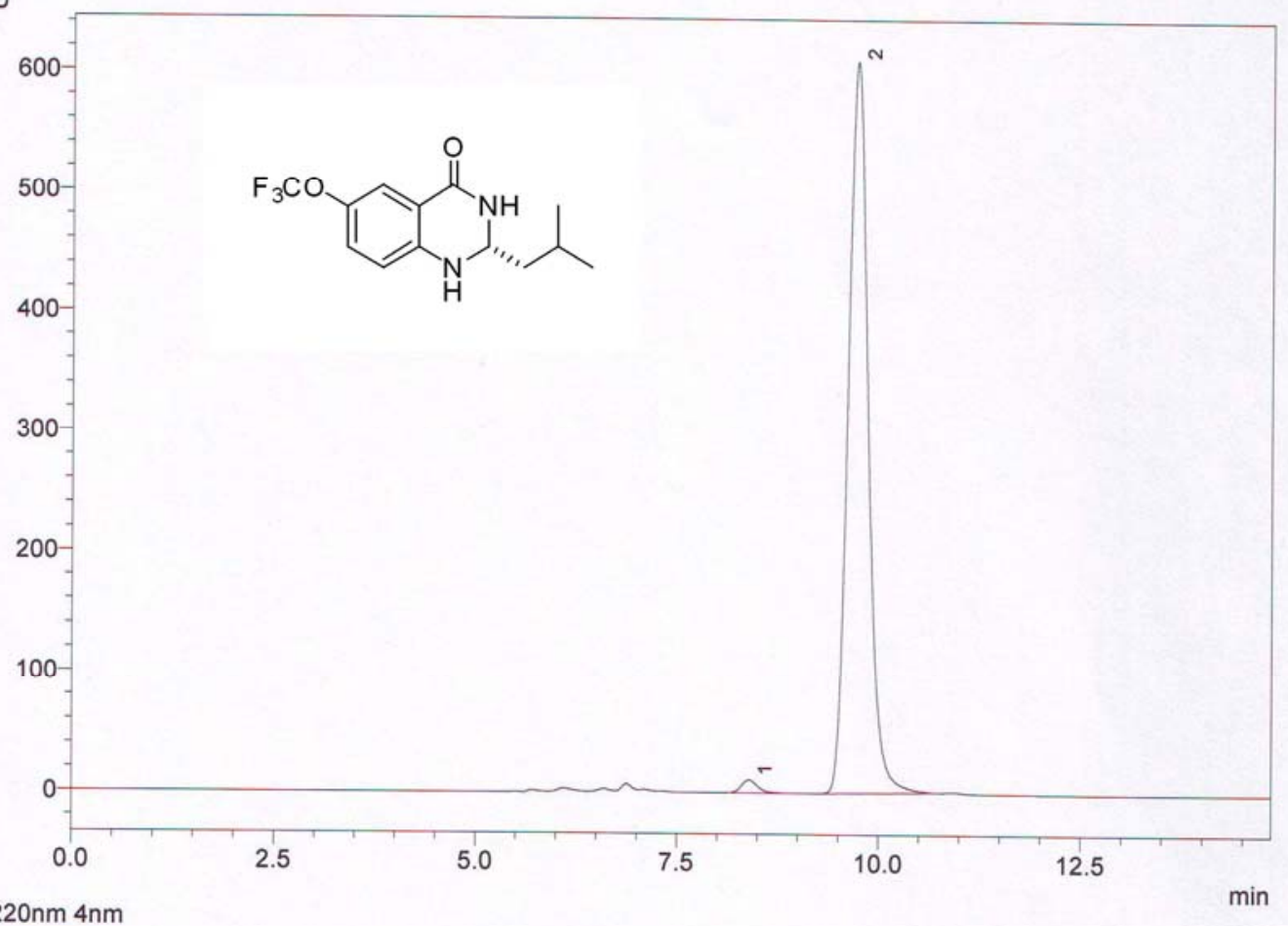

$1220 \mathrm{~nm} 4 \mathrm{~nm}$

PDA Ch1 220nm 4nm

Peak \# Ret. Time

Area \%

1

8.39

Total

1.39

98.61

S-70 


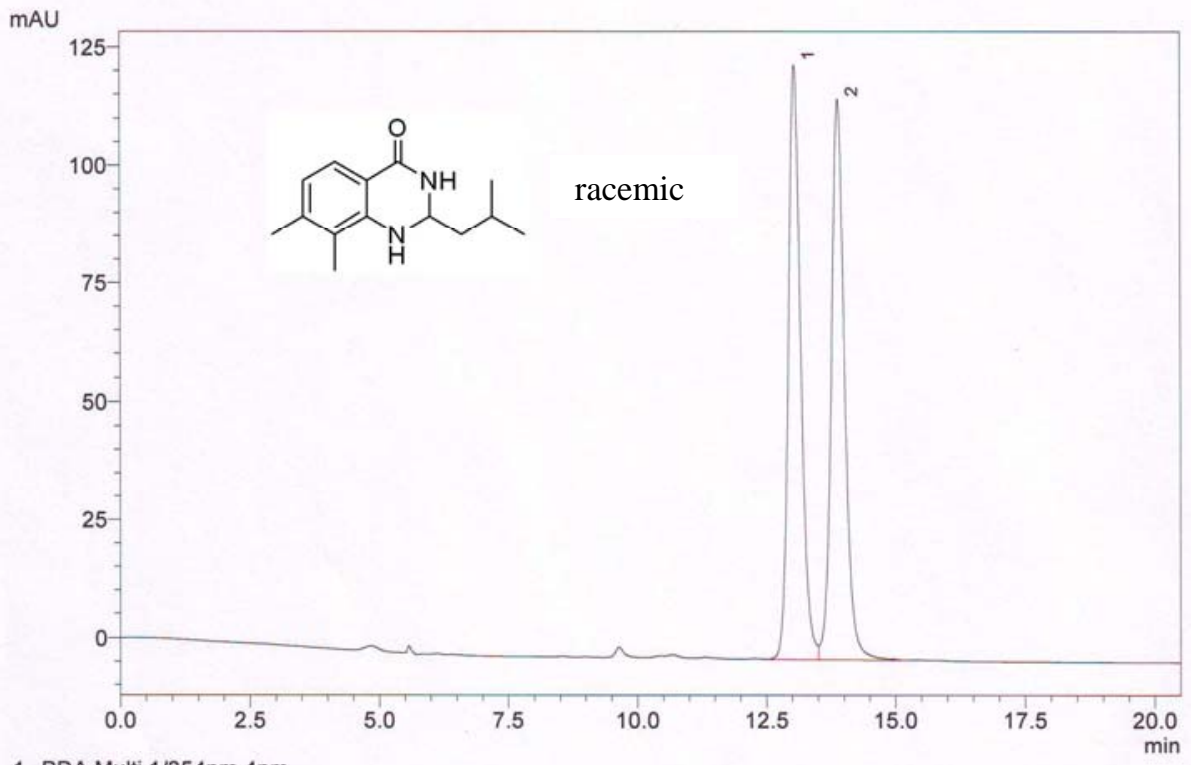

1 PDA Multi $1 / 254 \mathrm{~nm} 4 \mathrm{~nm}$

$\begin{array}{ccccr}\text { PDA Ch1 } 254 \mathrm{~nm} \text { 4nm } & & & \\ \text { Peak \# } & \text { Ret. Time } & \text { Area \% } & \text { Theoretical Plates } & \mathbf{k}^{\prime} \\ 1 & 13.04 & 49.42 & 14275 & 0.00 \\ 2 & 13.88 & 50.58 & 14136 & 0.06 \\ \text { Total } & & 100.00 & & \end{array}$

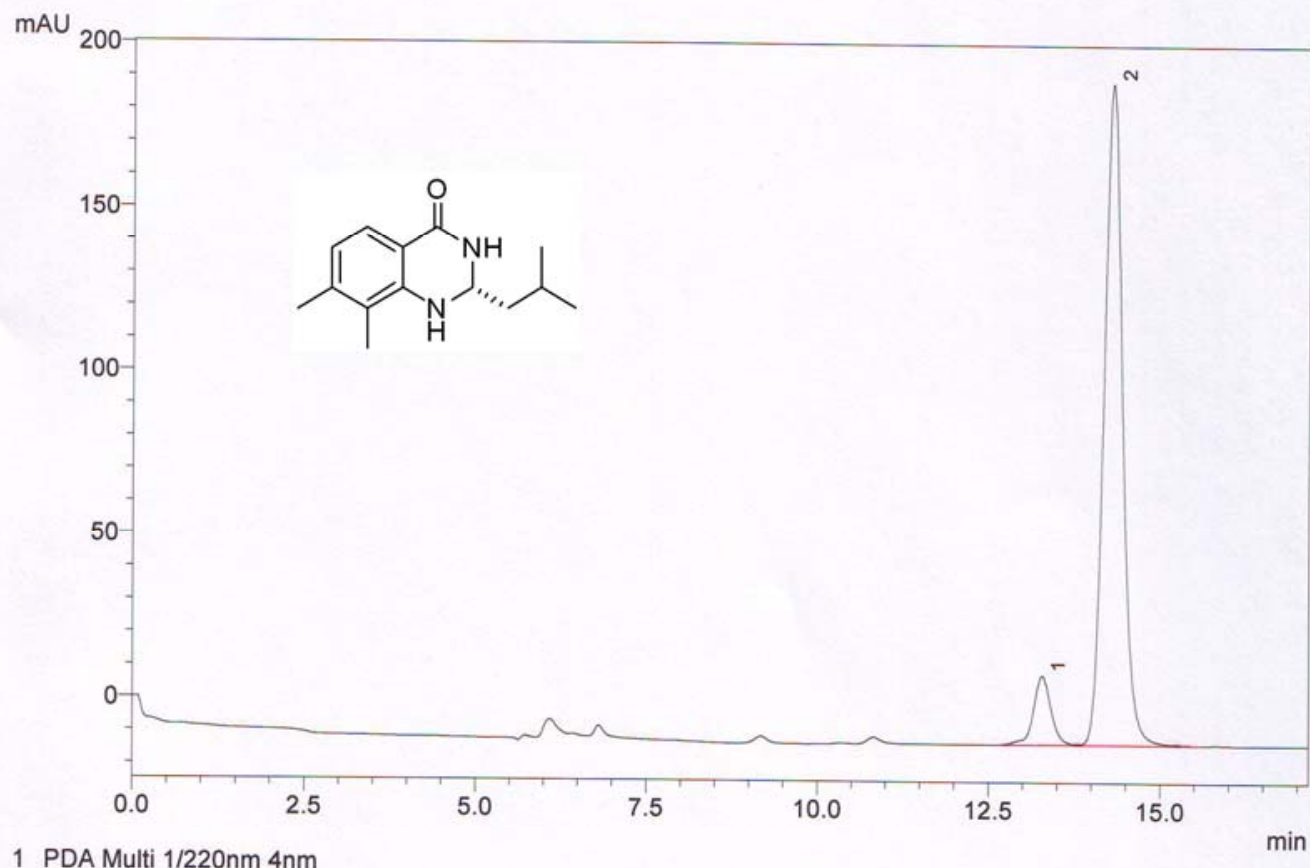

$\begin{array}{ccccr}\text { PDA Ch1 } 220 \mathrm{~nm} 4 \mathrm{~nm} & & & \\ \text { Peak \# } & \text { Ret. Time } & \text { Area \% } & \text { Theoretical Plates } & \mathbf{k}^{\prime} \\ 1 & 13.28 & 9.19 & 13397 & 0.00 \\ 2 & 14.28 & 90.81 & 13614 & 0.08 \\ \text { Total } & & 100.00 & & \end{array}$



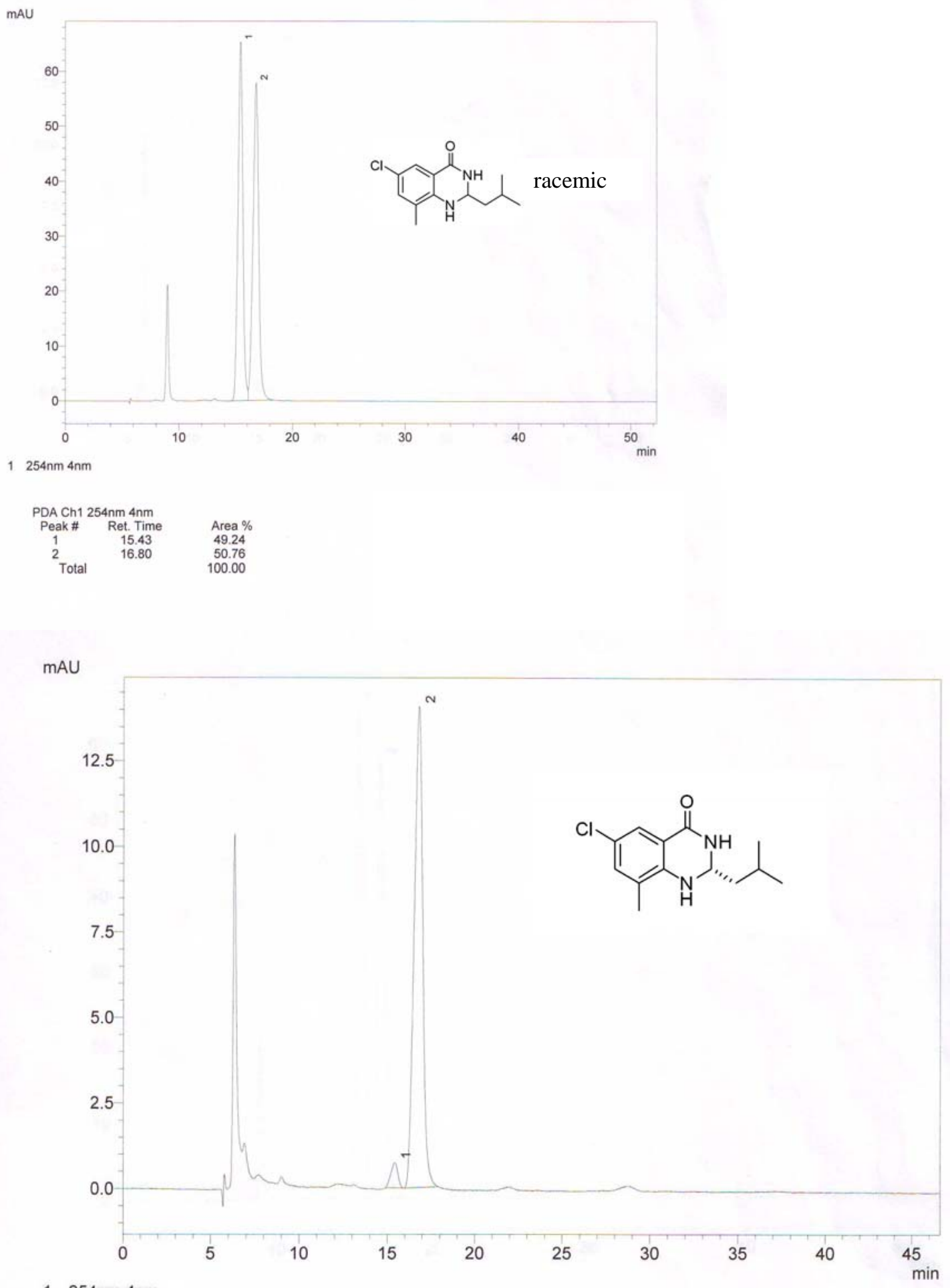

$1254 \mathrm{~nm} 4 \mathrm{~nm}$

PDA Ch1 254nm 4nm

Peak \# Ret. Time Area \%

$\begin{array}{llr}1 & 15.44 & 4.02 \\ 2 & 16.79 & 95.98 \\ \text { Total } & & 100.00\end{array}$




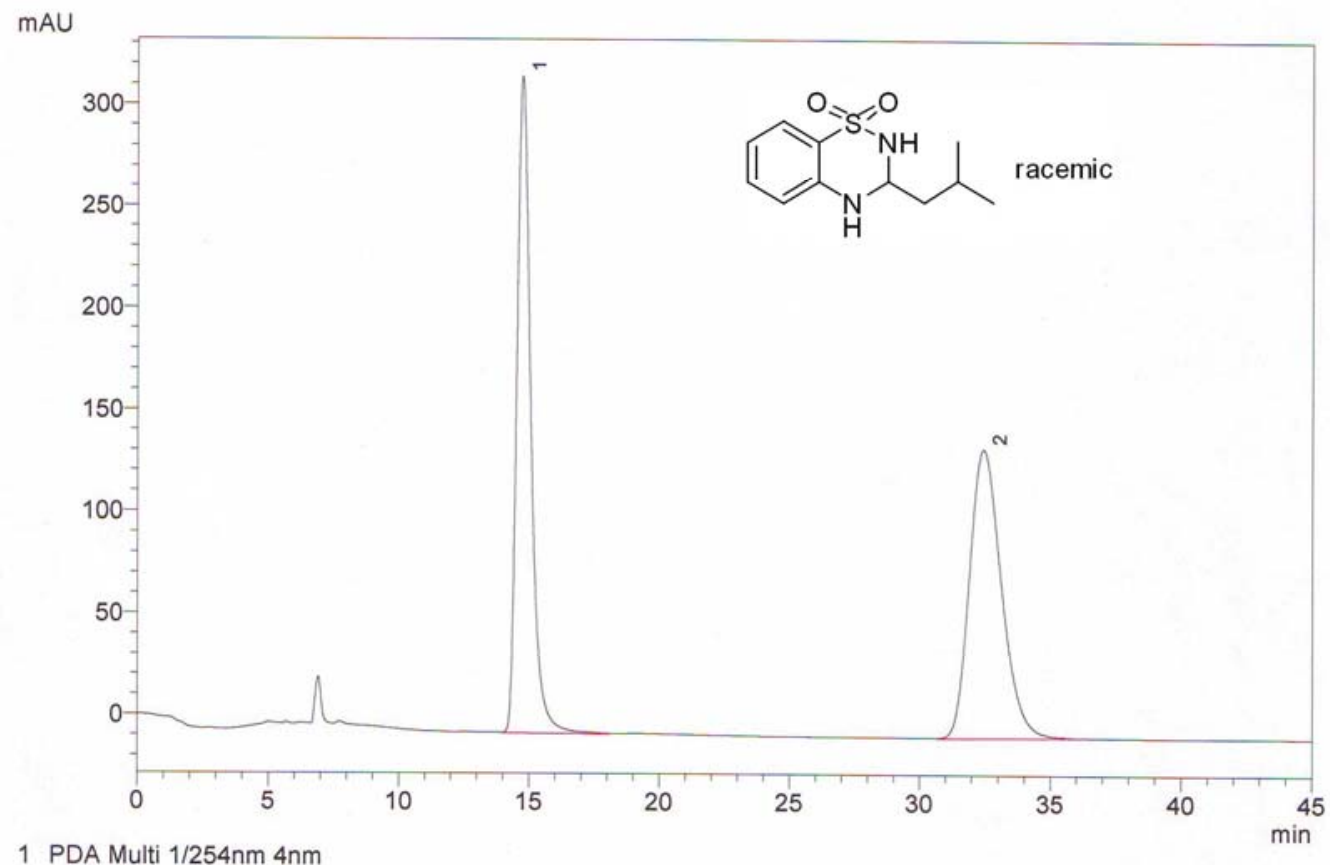

1 PDA Multi $1 / 254 \mathrm{~nm} 4 \mathrm{~nm}$

$\begin{array}{ccccr}\text { PDA Ch1 } 254 \mathrm{~nm} 4 \mathrm{~nm} & & & \\ \text { Peak \# } & \text { Ret. Time } & \text { Area \% } & \text { Theoretical Plates } & \mathrm{k}^{\prime} \\ 1 & 14.72 & 49.85 & 3878 & 0.00 \\ 2 & 32.43 & 50.15 & 3375 & 1.20 \\ \text { Total } & & 100.00 & & \end{array}$

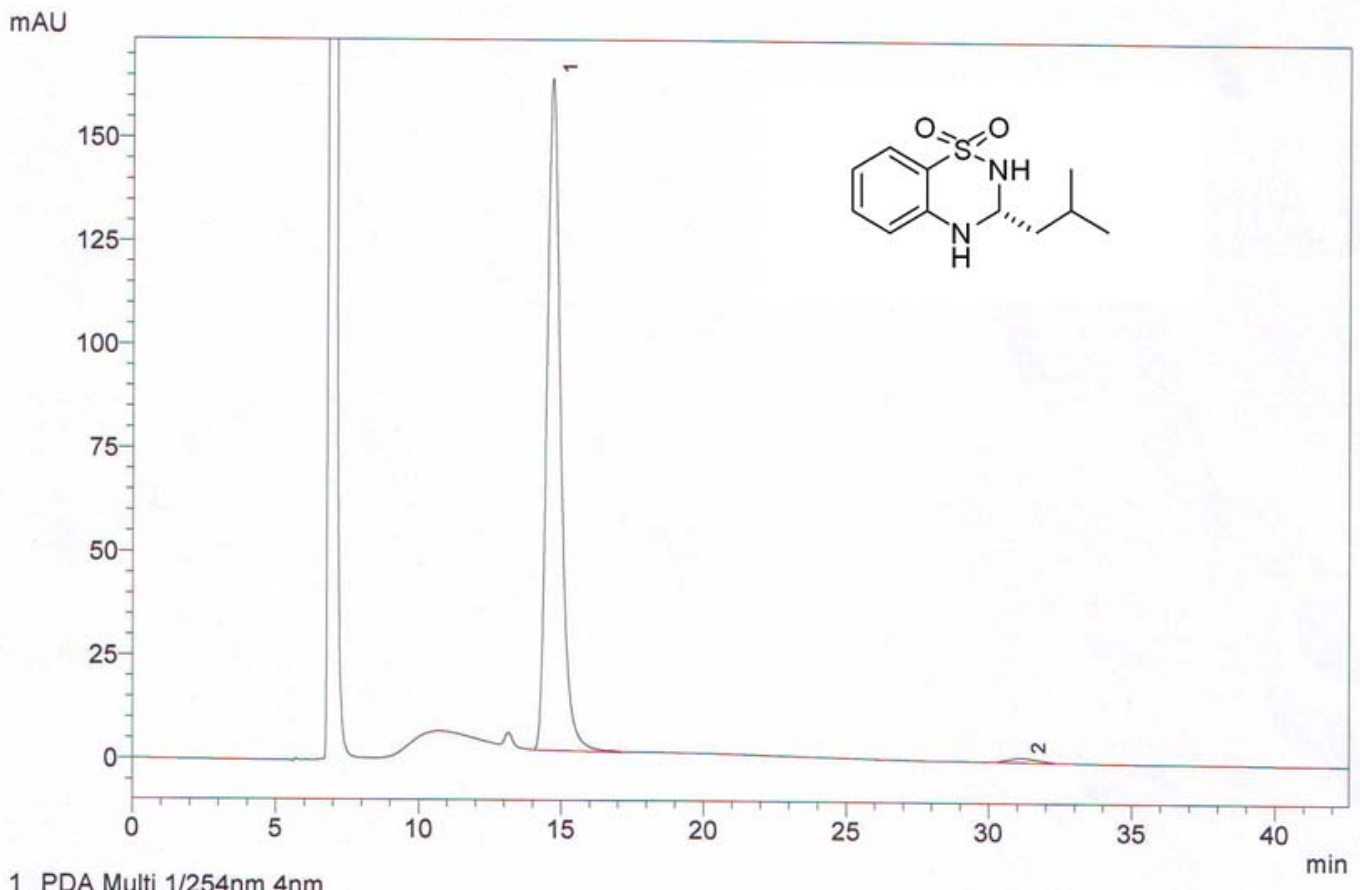

1 PDA Multi $1 / 254 \mathrm{~nm} 4 \mathrm{~nm}$

PDA Ch1 254nm 4nm

$\begin{array}{ccccr}\text { Peak \# } & \text { Ret. Time } & \text { Area \% } & \text { Theoretical Plates } & \text { k' }^{\prime} \\ 1 & 14.66 & 98.81 & 4676 & 0.00 \\ 2 & 31.15 & 1.19 & 4478 & 1.12 \\ \text { Total } & & 100.00 & & \end{array}$



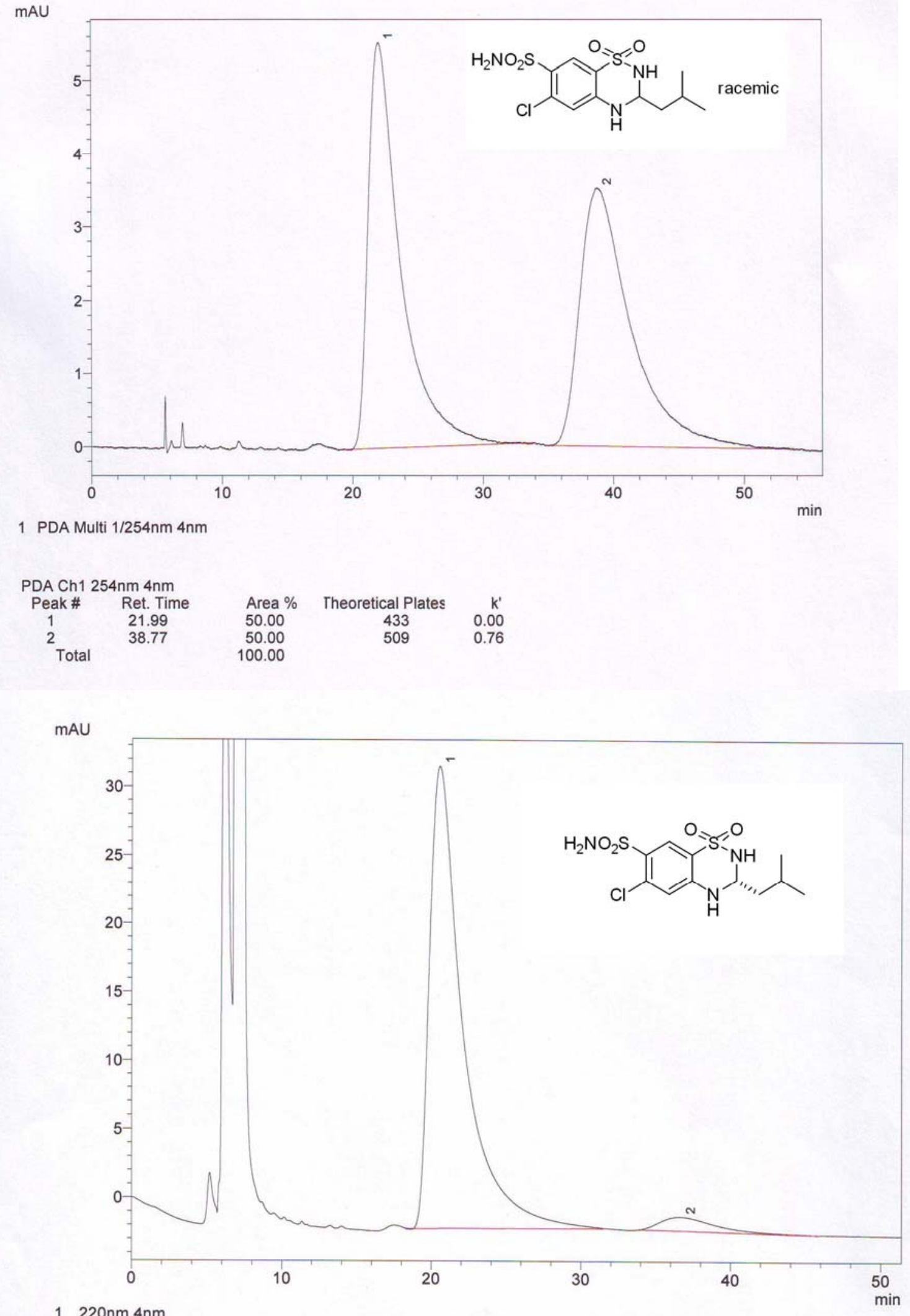

$1220 \mathrm{~nm} 4 \mathrm{~nm}$

PDA Ch1 220nm 4nm

$\begin{array}{ccc}\text { Peak \# } & \text { Ret. Time } & \text { Area } \% \\ 1 & 20.53 & 95.50 \\ 2 & 36.64 & 4.50 \\ \text { Total } & & 100.00\end{array}$




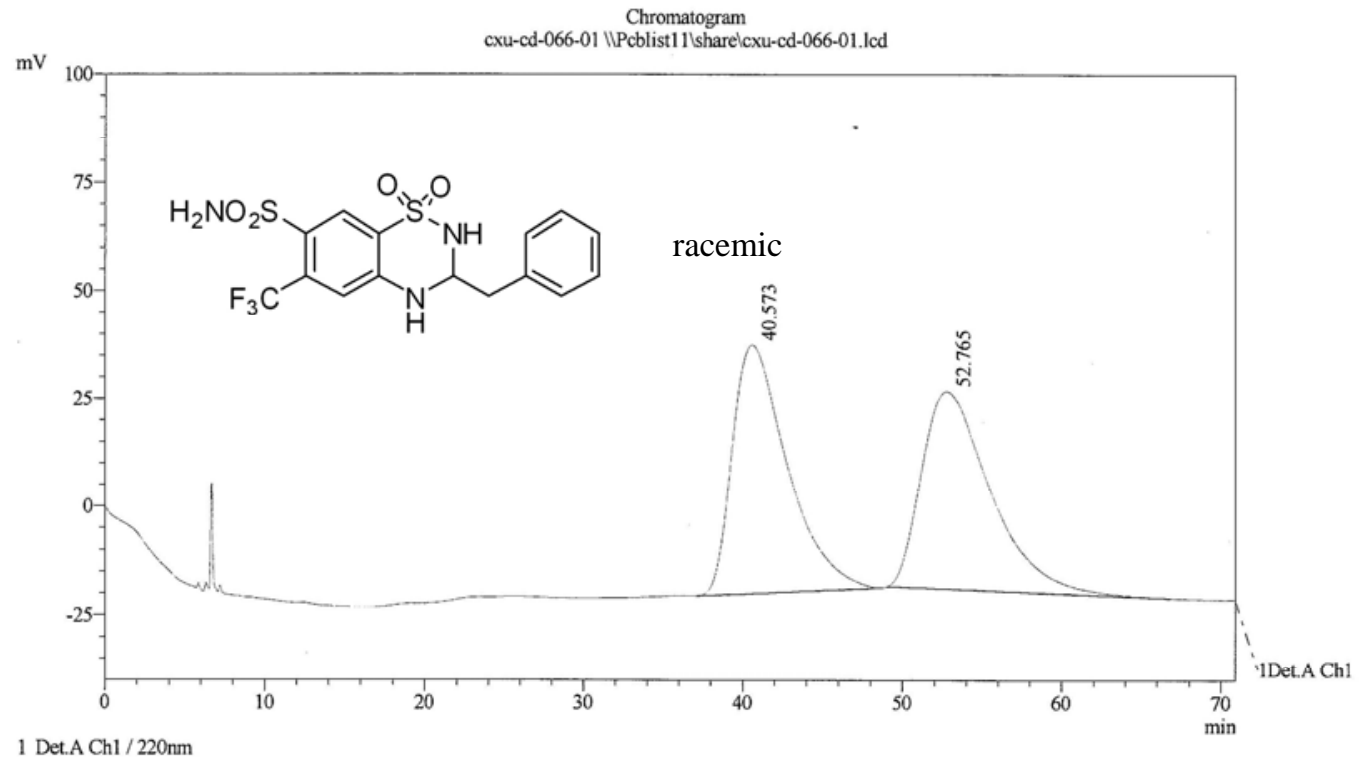

1 Det.A Chl / 220nm
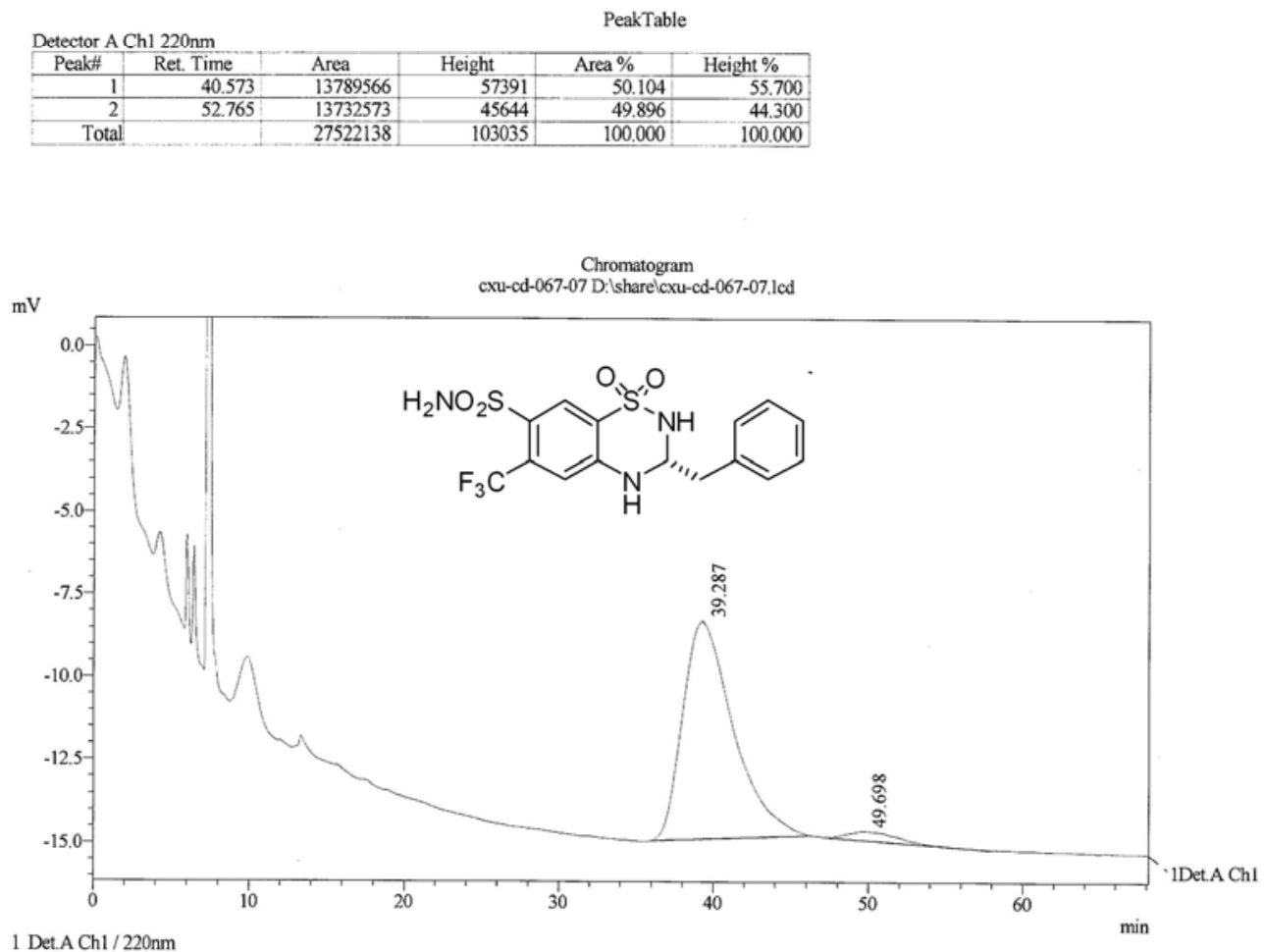

1 Det.A Chl / 220nm

\begin{tabular}{|c|c|c|c|c|c|}
\hline \multicolumn{6}{|c|}{ Detector A Ch1 220nm } \\
\hline Peak\# & Ret. Time & Area & Height & Area $\%$ & Height $\%$ \\
\hline 1 & 39.287 & 1485560 & 6565 & 96.070 & 96.024 \\
\hline 2 & 49.698 & 60779 & 272 & 3.930 & 3.976 \\
\hline Totes & & 1546338 & 6837 & 100.000 & 100.000 \\
\hline
\end{tabular}




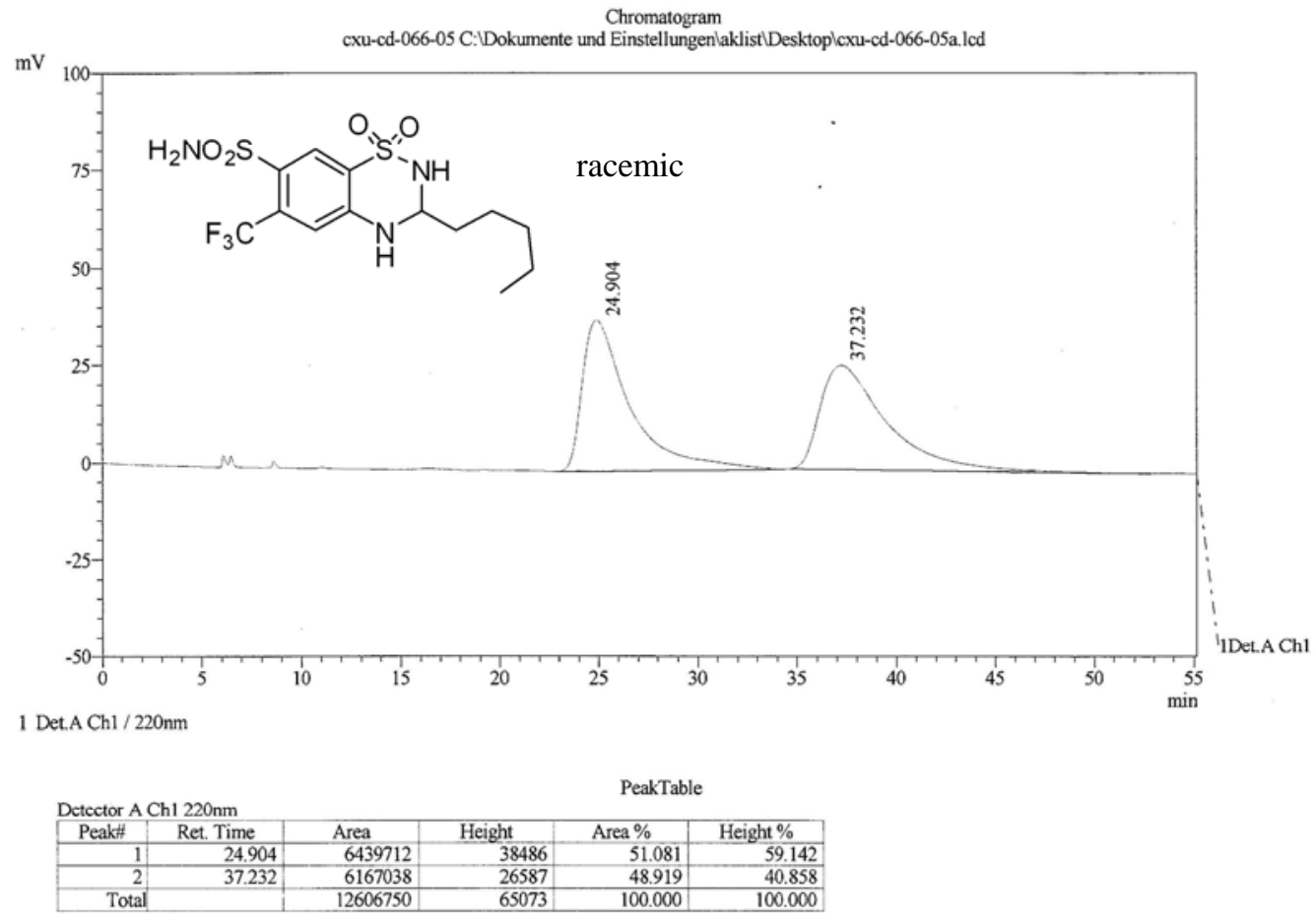

Chromatogram

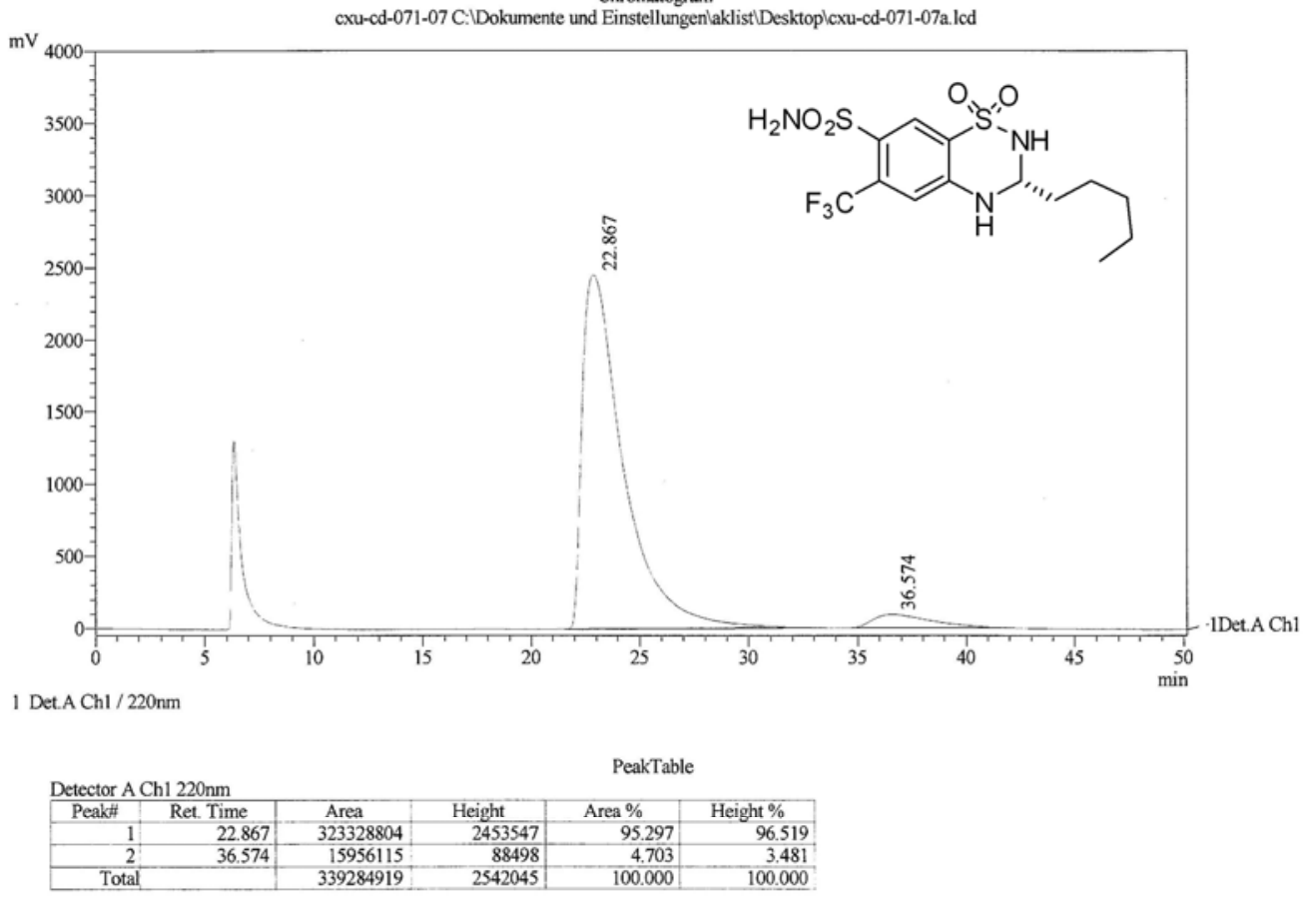

S-76 


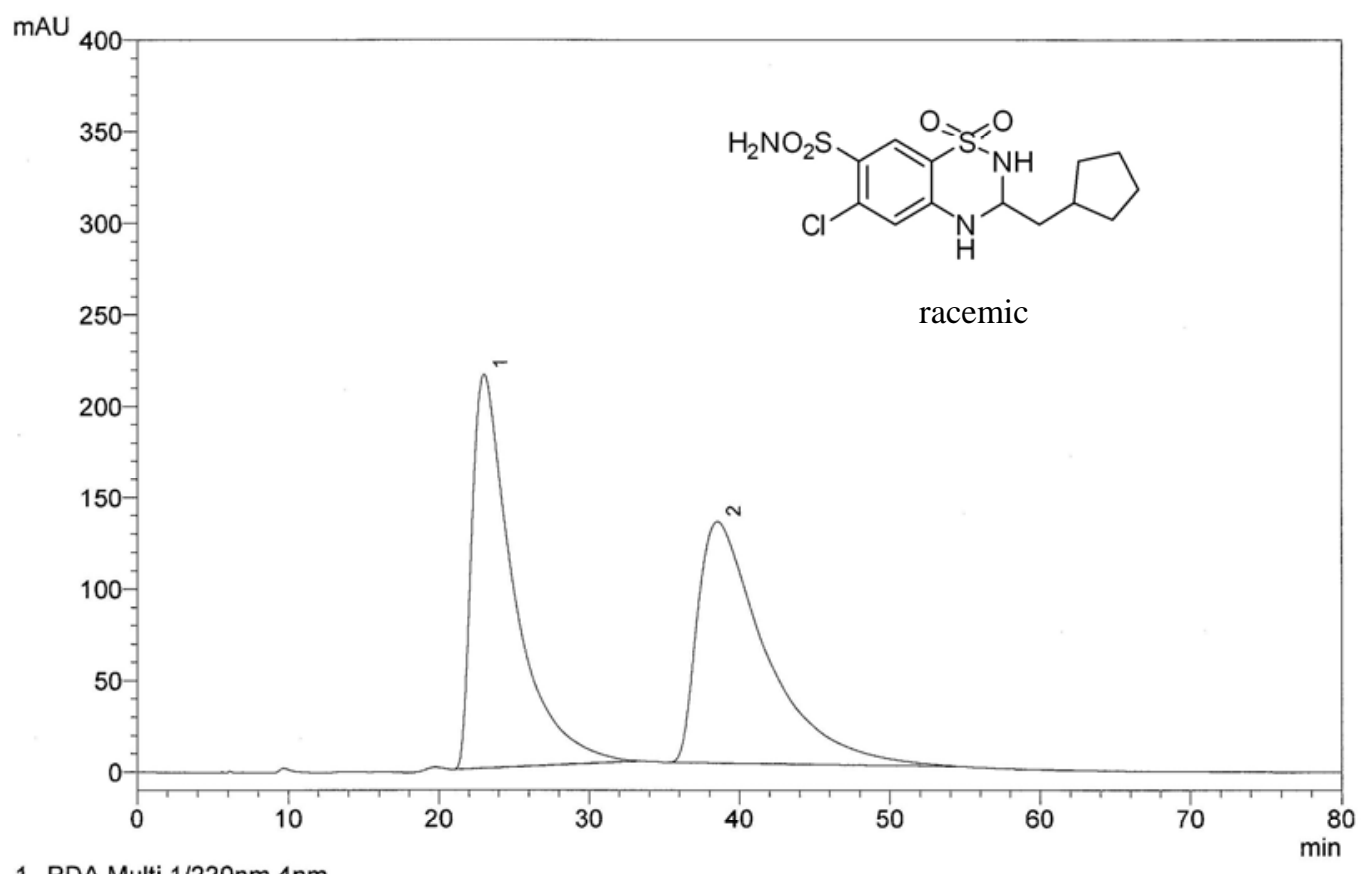

1 PDA Multi $1 / 220 \mathrm{~nm} 4 \mathrm{~nm}$

$\begin{array}{ccccr}\text { PDA Ch1 } 220 \mathrm{~nm} \text { 4nm } & & & \\ \text { Peak \# } & \text { Ret. Time } & \text { Area \% } & \text { Theoretical Plates } & \text { k' }^{\prime} \\ 1 & 22.99 & 50.02 & 408 & 0.00 \\ 2 & 38.51 & 49.98 & 385 & 0.68 \\ \text { Total } & & 100.00 & & \end{array}$

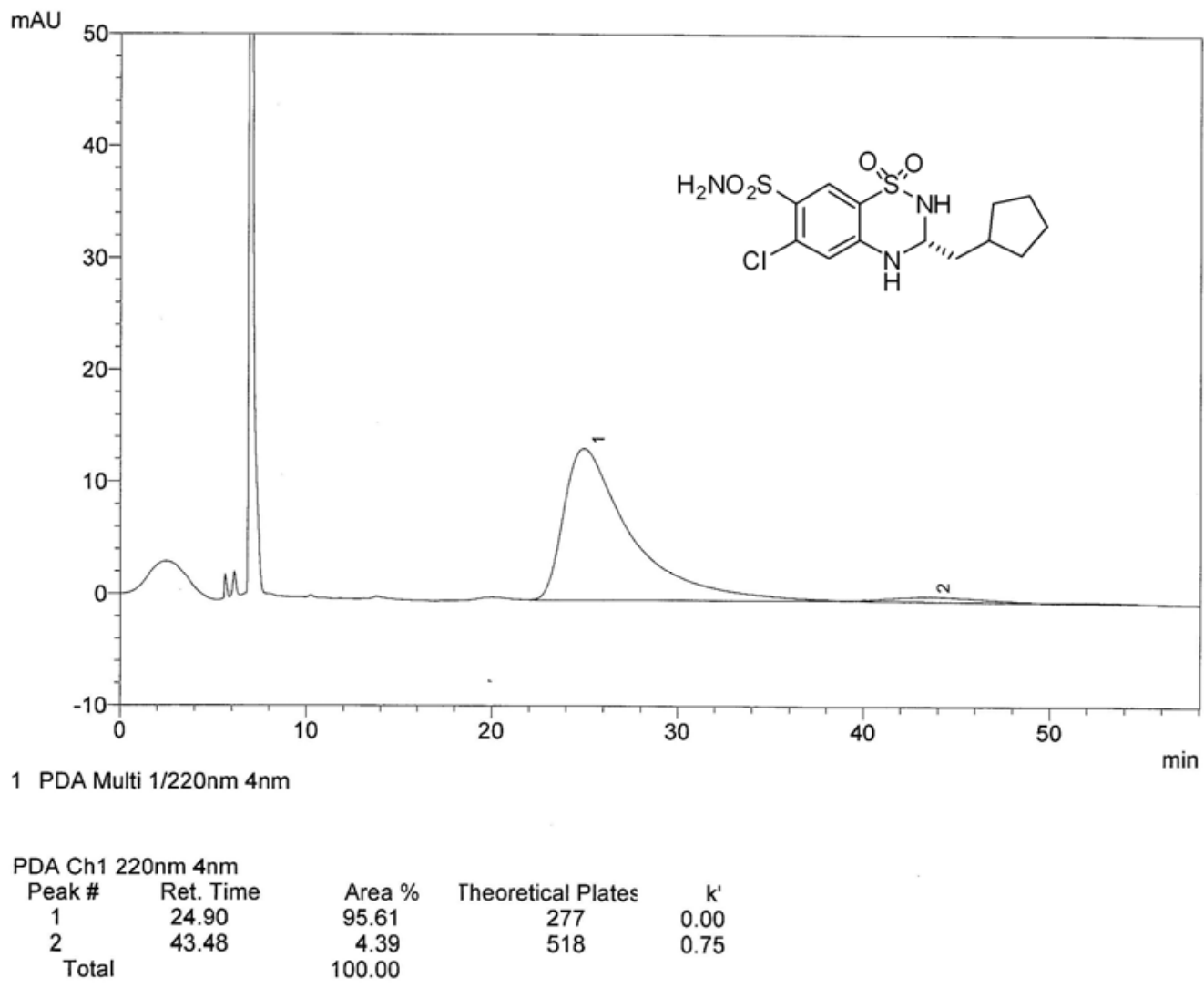




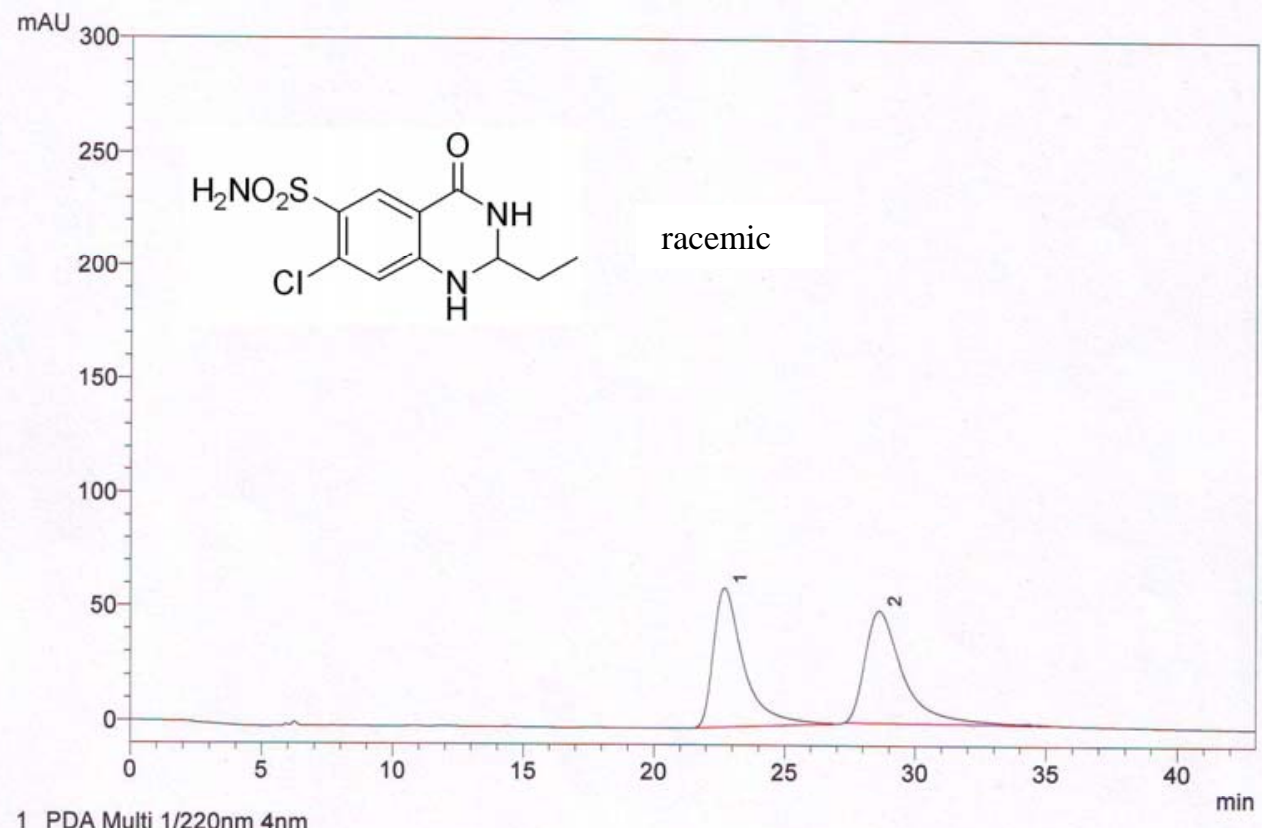

1 PDA Multi 1/220nm 4nm

$\begin{array}{ccccr}\text { PDA Ch1 } 220 \mathrm{~nm} \text { 4nm } & & & \\ \text { Peak \# } & \text { Ret. Time } & \text { Area \% } & \text { Theoretical Plates } & \text { k' }^{\prime} \\ 1 & 22.69 & 49.46 & 2225 & 0.00 \\ 2 & 28.61 & 50.54 & 2224 & 0.26 \\ \text { Total } & & 100.00 & & \end{array}$

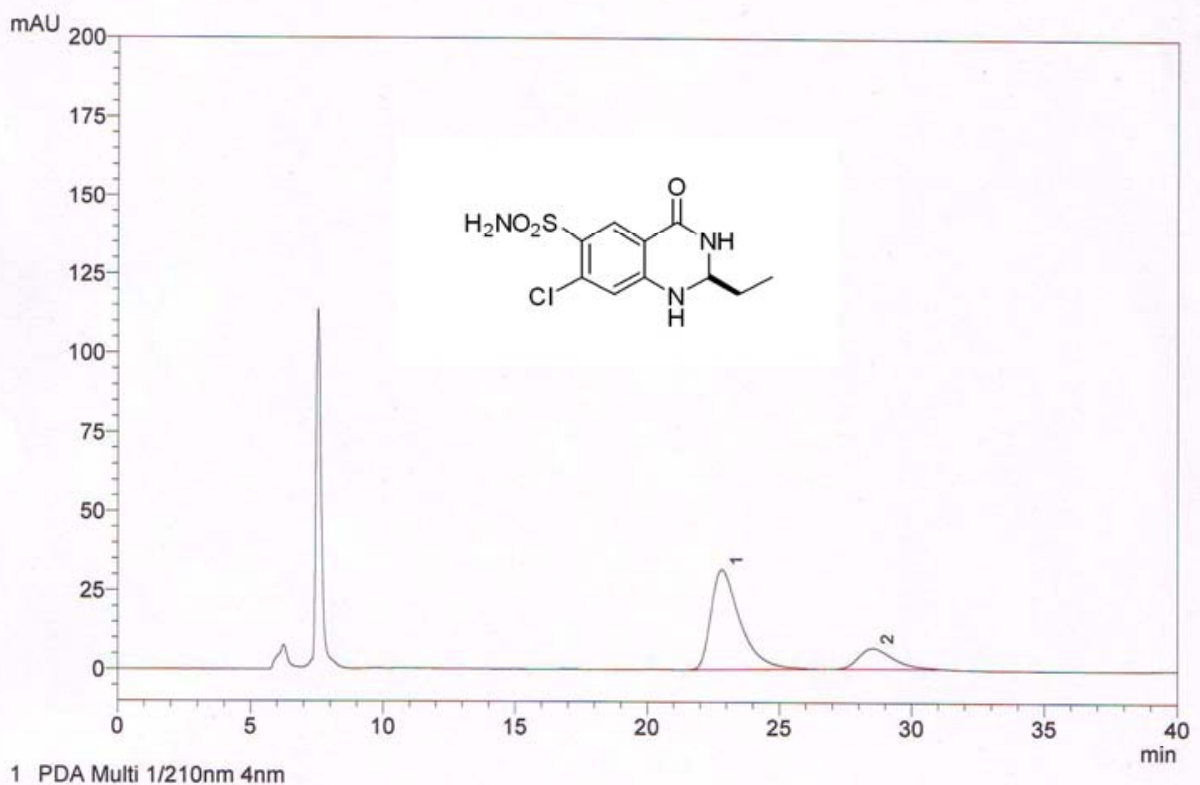

1 PDA Multi $1 / 210 \mathrm{~nm} 4 \mathrm{~nm}$

\begin{tabular}{ccccr} 
PDA Ch1 & \multicolumn{2}{l}{ 210nm 4nm } & & \\
Peak \# & Ret. Time & Area \% & Theoretical Plates & $k^{\prime}$ \\
1 & 22.81 & 80.44 & 2230 & 0.00 \\
2 & 28.52 & 19.56 & 2401 & 0.25 \\
Total & & 100.00 & &
\end{tabular}




\section{Preparation and Analysis of Single Crystal of Compound 3k}

A solution of compound 3k (20 mg, 98:2 er) in $5 \mathrm{~mL}$ of DCM in an open $10 \mathrm{~mL}$-sample vial was left alone for 2 days at room temperature in the open air to provide suitable crystals. Two pieces of crystals were analyzed by Röntgen-diffraction and confirmed the absolute configuration as $R$. 
Table 1. Crystal data and structure refinement.

Identification code

Empirical formula

Color

Formula weight

Temperature

Wavelength

Crystal system

Space group

Unit cell dimensions

Volume

Z

Density (calculated)

Absorption coefficient

$\mathrm{F}(000)$

Crystal size

$\theta$ range for data collection

Index ranges

Reflections collected

Independent reflections

Reflections with $\mathrm{I}>2 \sigma(\mathrm{I})$

Completeness to $\theta=69.56^{\circ}$

Absorption correction

Max. and min. transmission

Refinement method

Data / restraints / parameters

Goodness-of-fit on $\mathrm{F}^{2}$

Final $R$ indices $[I>2 \sigma(I)]$

$\mathrm{R}$ indices (all data)

Absolute structure parameter

Largest diff. peak and hole
6263

$\mathrm{C}_{12} \mathrm{H}_{15} \mathrm{ClN}_{2} \mathrm{O}$

colourless

$238.71 \mathrm{~g} \cdot \mathrm{mol}^{-1}$

$100 \mathrm{~K}$

$1.54178 \AA$

Orthorhombic

$\mathbf{P 2} \mathbf{2}_{1} \mathbf{2}_{1}$, (no. 19)

$\mathrm{a}=9.6888(5) \AA \quad \alpha=90.0^{\circ}$.

$\mathrm{b}=11.7838(7) \AA \quad \beta=90.0^{\circ}$.

$\mathrm{c}=21.5338(12) \AA \quad \gamma=90.0^{\circ}$.

$2458.5(2) \AA^{3}$

8

$1.290 \mathrm{Mg} \cdot \mathrm{m}^{-3}$

$2.596 \mathrm{~mm}^{-1}$

$1008 \mathrm{e}$

$0.38 \times 0.21 \times 0.04 \mathrm{~mm}^{3}$

4.11 to $69.56^{\circ}$.

$-11 \leq \mathrm{h} \leq 11,-13 \leq \mathrm{k} \leq 13,-25 \leq 1 \leq 25$

53830

$4510\left[\mathrm{R}_{\mathrm{int}}=0.1002\right]$

3153

$97.7 \%$

Gaussian

0.89980 and 0.44762

Full-matrix least-squares on $\mathrm{F}^{2}$

$4510 / 0 / 303$

1.083

$\mathrm{R}_{1}=0.0781$

$\mathrm{wR}^{2}=0.1946$

$\mathrm{R}_{1}=0.1195$

$\mathrm{wR}^{2}=0.2286$

$0.01(4)$

0.480 and $-0.513 \mathrm{e} \cdot \AA^{-3}$ 
Table 2. Atomic coordinates and equivalent isotropic displacement parameters $\left(\AA^{2}\right)$. $\mathrm{U}_{\mathrm{eq}}$ is defined as one third of the trace of the orthogonalized $\mathrm{U}_{\mathrm{ij}}$ tensor.

\begin{tabular}{|c|c|c|c|c|}
\hline & $\mathrm{x}$ & $\mathrm{y}$ & $\mathrm{z}$ & $\mathrm{U}_{\mathrm{eq}}$ \\
\hline $\mathrm{C}(1)$ & $0.3424(5)$ & $0.0100(5)$ & $0.5686(3)$ & $0.045(1)$ \\
\hline $\mathrm{C}(2)$ & $0.2182(6)$ & $0.1440(6)$ & $0.5018(3)$ & $0.052(2)$ \\
\hline$C(3)$ & $0.1650(5)$ & $0.1387(5)$ & $0.6125(3)$ & $0.050(1)$ \\
\hline$C(4)$ & $0.0796(6)$ & $0.1746(6)$ & $0.6625(3)$ & $0.062(2)$ \\
\hline$C(5)$ & $0.0743(6)$ & $0.1086(7)$ & $0.7148(3)$ & $0.066(2)$ \\
\hline$C(6)$ & $0.1513(6)$ & $0.0119(6)$ & $0.7209(3)$ & $0.062(2)$ \\
\hline$C(7)$ & $0.2352(6)$ & $-0.0243(6)$ & $0.6732(3)$ & $0.052(2)$ \\
\hline$C(8)$ & $0.2417(5)$ & $0.0386(5)$ & $0.6181(3)$ & $0.046(1)$ \\
\hline $\mathrm{C}(9)$ & $0.2588(6)$ & $0.2249(5)$ & $0.4490(3)$ & $0.052(2)$ \\
\hline$C(10)$ & $0.1429(6)$ & $0.2936(6)$ & $0.4219(3)$ & $0.060(2)$ \\
\hline $\mathrm{C}(11)$ & $0.0327(7)$ & $0.2188(7)$ & $0.3926(3)$ & $0.069(2)$ \\
\hline$C(12)$ & $0.2016(8)$ & $0.3737(7)$ & $0.3735(3)$ & $0.073(2)$ \\
\hline$C(13)$ & $0.1384(6)$ & $0.5204(5)$ & $0.5674(3)$ & $0.047(1)$ \\
\hline$C(14)$ & $0.2242(7)$ & $0.6982(6)$ & $0.5186(3)$ & $0.066(2)$ \\
\hline$C(15)$ & $0.3128(7)$ & $0.6388(6)$ & $0.6184(3)$ & $0.064(2)$ \\
\hline$C(16)$ & $0.4062(7)$ & $0.6598(7)$ & $0.6659(4)$ & $0.071(2)$ \\
\hline$C(17)$ & $0.4286(6)$ & $0.5781(6)$ & $0.7105(3)$ & $0.064(2)$ \\
\hline$C(18)$ & $0.3568(6)$ & $0.4785(6)$ & $0.7087(3)$ & $0.055(2)$ \\
\hline$C(19)$ & $0.2629(6)$ & $0.4563(5)$ & $0.6620(3)$ & $0.048(1)$ \\
\hline$C(20)$ & $0.2417(5)$ & $0.5391(5)$ & $0.6170(3)$ & $0.045(1)$ \\
\hline $\mathrm{C}(21)$ & $0.3354(11)$ & $0.6797(9)$ & $0.4688(5)$ & $0.110(3)$ \\
\hline $\mathrm{C}(22 \mathrm{~A})$ & $0.3011(8)$ & $0.6886(8)$ & $0.4063(4)$ & $0.048(3)$ \\
\hline $\mathrm{C}(22 \mathrm{~B})$ & $0.362(2)$ & $0.769(3)$ & $0.4252(11)$ & $0.095(12)$ \\
\hline $\mathrm{C}(23)$ & $0.2642(10)$ & $0.8190(9)$ & $0.3903(5)$ & $0.110(3)$ \\
\hline$C(24)$ & $0.4264(9)$ & $0.6528(12)$ & $0.3655(5)$ & $0.130(4)$ \\
\hline $\mathrm{Cl}(1)$ & $0.1388(2)$ & $-0.0722(2)$ & $0.7871(1)$ & $0.088(1)$ \\
\hline $\mathrm{Cl}(2)$ & $0.3891(2)$ & $0.3749(2)$ & $0.7641(1)$ & $0.084(1)$ \\
\hline$N(1)$ & $0.3340(5)$ & $0.0732(4)$ & $0.5170(2)$ & $0.049(1)$ \\
\hline $\mathrm{N}(2)$ & $0.1757(5)$ & $0.2020(5)$ & $0.5576(3)$ & $0.061(1)$ \\
\hline $\mathrm{N}(3)$ & $0.1333(6)$ & $0.5983(4)$ & $0.5220(2)$ & $0.060(1)$ \\
\hline$N(4)$ & $0.2822(8)$ & $0.7219(6)$ & $0.5754(4)$ & $0.095(2)$ \\
\hline $\mathrm{O}(1)$ & $0.4314(4)$ & $-0.0646(3)$ & $0.5752(2)$ & $0.048(1)$ \\
\hline $\mathrm{O}(2)$ & $0.0610(4)$ & $0.4351(4)$ & $0.5668(2)$ & $0.049(1)$ \\
\hline
\end{tabular}


Table 3. Bond lengths $[\AA]$ and angles $\left[^{\circ}\right]$.

\begin{tabular}{|c|c|c|c|}
\hline$C(1)-O(1)$ & $1.240(7)$ & $\mathrm{C}(1)-\mathrm{N}(1)$ & $1.341(7)$ \\
\hline$C(1)-C(8)$ & $1.485(8)$ & $\mathrm{C}(2)-\mathrm{N}(1)$ & $1.436(7)$ \\
\hline $\mathrm{C}(2)-\mathrm{N}(2)$ & $1.441(8)$ & $\mathrm{C}(2)-\mathrm{C}(9)$ & $1.536(8)$ \\
\hline$C(3)-C(8)$ & $1.399(8)$ & $\mathrm{C}(3)-\mathrm{N}(2)$ & $1.402(8)$ \\
\hline$C(3)-C(4)$ & $1.422(9)$ & $\mathrm{C}(4)-\mathrm{C}(5)$ & $1.370(10)$ \\
\hline$C(5)-C(6)$ & $1.368(10)$ & $C(6)-C(7)$ & $1.378(9)$ \\
\hline $\mathrm{C}(6)-\mathrm{Cl}(1)$ & $1.741(7)$ & $C(7)-C(8)$ & $1.399(8)$ \\
\hline $\mathrm{C}(9)-\mathrm{C}(10)$ & $1.501(8)$ & $C(10)-C(12)$ & $1.516(10)$ \\
\hline$C(10)-C(11)$ & $1.521(9)$ & $\mathrm{C}(13)-\mathrm{O}(2)$ & $1.254(7)$ \\
\hline $\mathrm{C}(13)-\mathrm{N}(3)$ & $1.342(7)$ & $C(13)-C(20)$ & $1.480(8)$ \\
\hline $\mathrm{C}(14)-\mathrm{N}(4)$ & $1.375(10)$ & $\mathrm{C}(14)-\mathrm{N}(3)$ & $1.472(9)$ \\
\hline$C(14)-C(21)$ & $1.535(12)$ & $C(15)-C(20)$ & $1.362(9)$ \\
\hline $\mathrm{C}(15)-\mathrm{N}(4)$ & $1.379(9)$ & $C(15)-C(16)$ & $1.389(10)$ \\
\hline $\mathrm{C}(16)-\mathrm{C}(17)$ & $1.377(10)$ & $C(17)-C(18)$ & $1.365(9)$ \\
\hline$C(18)-C(19)$ & $1.382(8)$ & $\mathrm{C}(18)-\mathrm{Cl}(2)$ & $1.736(7)$ \\
\hline$C(19)-C(20)$ & $1.390(8)$ & $C(21)-C(22 A)$ & $1.391(13)$ \\
\hline$C(22 \mathrm{~A})-\mathrm{C}(24)$ & $1.557(12)$ & $C(22 A)-C(23)$ & $1.615(14)$ \\
\hline $\mathrm{O}(1)-\mathrm{C}(1)-\mathrm{N}(1)$ & $122.1(5)$ & $\mathrm{O}(1)-\mathrm{C}(1)-\mathrm{C}(8)$ & $122.4(5)$ \\
\hline $\mathrm{N}(1)-\mathrm{C}(1)-\mathrm{C}(8)$ & $115.4(5)$ & $\mathrm{N}(1)-\mathrm{C}(2)-\mathrm{N}(2)$ & $108.0(5)$ \\
\hline $\mathrm{N}(1)-\mathrm{C}(2)-\mathrm{C}(9)$ & $109.2(5)$ & $\mathrm{N}(2)-\mathrm{C}(2)-\mathrm{C}(9)$ & $113.3(5)$ \\
\hline $\mathrm{C}(8)-\mathrm{C}(3)-\mathrm{N}(2)$ & $118.8(5)$ & $C(8)-C(3)-C(4)$ & $119.6(6)$ \\
\hline $\mathrm{N}(2)-\mathrm{C}(3)-\mathrm{C}(4)$ & $121.6(6)$ & $C(5)-C(4)-C(3)$ & $118.3(6)$ \\
\hline$C(6)-C(5)-C(4)$ & $122.2(6)$ & $C(5)-C(6)-C(7)$ & $120.5(6)$ \\
\hline $\mathrm{C}(5)-\mathrm{C}(6)-\mathrm{Cl}(1)$ & $121.0(6)$ & $\mathrm{C}(7)-\mathrm{C}(6)-\mathrm{Cl}(1)$ & $118.4(6)$ \\
\hline $\mathrm{C}(6)-\mathrm{C}(7)-\mathrm{C}(8)$ & $119.6(6)$ & $C(3)-C(8)-C(7)$ & $119.8(5)$ \\
\hline$C(3)-C(8)-C(1)$ & $118.5(5)$ & $\mathrm{C}(7)-\mathrm{C}(8)-\mathrm{C}(1)$ & $121.2(5)$ \\
\hline $\mathrm{C}(10)-\mathrm{C}(9)-\mathrm{C}(2)$ & $115.5(5)$ & $C(9)-C(10)-C(12)$ & $108.8(5)$ \\
\hline$C(9)-C(10)-C(11)$ & $111.9(6)$ & $C(12)-C(10)-C(11)$ & $109.8(6)$ \\
\hline $\mathrm{O}(2)-\mathrm{C}(13)-\mathrm{N}(3)$ & $121.2(5)$ & $\mathrm{O}(2)-\mathrm{C}(13)-\mathrm{C}(20)$ & $122.1(5)$ \\
\hline $\mathrm{N}(3)-\mathrm{C}(13)-\mathrm{C}(20)$ & $116.7(5)$ & $\mathrm{N}(4)-\mathrm{C}(14)-\mathrm{N}(3)$ & $111.2(6)$ \\
\hline $\mathrm{N}(4)-\mathrm{C}(14)-\mathrm{C}(21)$ & $111.3(8)$ & $\mathrm{N}(3)-\mathrm{C}(14)-\mathrm{C}(21)$ & $109.9(7)$ \\
\hline $\mathrm{C}(20)-\mathrm{C}(15)-\mathrm{N}(4)$ & $119.3(6)$ & $C(20)-C(15)-C(16)$ & $119.9(7)$ \\
\hline N(4)-C(15)-C(16) & $120.5(7)$ & $\mathrm{C}(17)-\mathrm{C}(16)-\mathrm{C}(15)$ & $119.5(7)$ \\
\hline$C(18)-C(17)-C(16)$ & $120.0(6)$ & $C(17)-C(18)-C(19)$ & $121.3(6)$ \\
\hline $\mathrm{C}(17)-\mathrm{C}(18)-\mathrm{Cl}(2)$ & $119.6(5)$ & $\mathrm{C}(19)-\mathrm{C}(18)-\mathrm{Cl}(2)$ & $119.0(5)$ \\
\hline $\mathrm{C}(18)-\mathrm{C}(19)-\mathrm{C}(20)$ & $118.1(6)$ & $\mathrm{C}(15)-\mathrm{C}(20)-\mathrm{C}(19)$ & $121.1(6)$ \\
\hline $\mathrm{C}(15)-\mathrm{C}(20)-\mathrm{C}(13)$ & $119.1(5)$ & $\mathrm{C}(19)-\mathrm{C}(20)-\mathrm{C}(13)$ & $119.9(5)$ \\
\hline
\end{tabular}




$\begin{array}{llll}\mathrm{C}(22 \mathrm{~A})-\mathrm{C}(21)-\mathrm{C}(14) & 119.9(9) & \mathrm{C}(21)-\mathrm{C}(22 \mathrm{~A})-\mathrm{C}(24) & 109.9(8) \\ \mathrm{C}(21)-\mathrm{C}(22 \mathrm{~A})-\mathrm{C}(23) & 109.3(9) & \mathrm{C}(24)-\mathrm{C}(22 \mathrm{~A})-\mathrm{C}(23) & 108.0(8) \\ \mathrm{C}(1)-\mathrm{N}(1)-\mathrm{C}(2) & 124.0(5) & \mathrm{C}(3)-\mathrm{N}(2)-\mathrm{C}(2) & 118.2(5) \\ \mathrm{C}(13)-\mathrm{N}(3)-\mathrm{C}(14) & 124.2(5) & \mathrm{C}(14)-\mathrm{N}(4)-\mathrm{C}(15) & 122.7(6)\end{array}$


Table 4. Anisotropic displacement parameters $\left(\AA^{2}\right)$.

The anisotropic displacement factor exponent takes the form:

$-2 \pi^{2}\left[h^{2} a^{* 2} U_{11}+\ldots+2 h k a^{*} b^{*} U_{12}\right]$.

\begin{tabular}{|c|c|c|c|c|c|c|}
\hline & $\mathrm{U}_{11}$ & $\mathrm{U}_{22}$ & $\mathrm{U}_{33}$ & $\mathrm{U}_{23}$ & $\mathrm{U}_{13}$ & $\mathrm{U}_{12}$ \\
\hline$C(1)$ & $0.032(3)$ & $0.057(4)$ & $0.045(3)$ & $0.002(3)$ & $-0.001(2)$ & $-0.010(3)$ \\
\hline $\mathrm{C}(2)$ & $0.038(3)$ & $0.062(4)$ & $0.057(4)$ & $0.011(3)$ & $-0.007(3)$ & $-0.006(3)$ \\
\hline$C(3)$ & $0.029(3)$ & $0.062(4)$ & $0.060(4)$ & $0.003(3)$ & $-0.001(2)$ & $-0.002(3)$ \\
\hline $\mathrm{C}(4)$ & $0.034(3)$ & $0.061(4)$ & $0.091(5)$ & $0.006(4)$ & $0.007(3)$ & $-0.004(3)$ \\
\hline$C(5)$ & $0.038(3)$ & $0.091(6)$ & $0.070(4)$ & $-0.001(4)$ & $0.010(3)$ & $-0.006(3)$ \\
\hline$C(6)$ & $0.041(3)$ & $0.081(5)$ & $0.064(4)$ & $0.013(3)$ & $0.002(3)$ & $-0.014(3)$ \\
\hline$C(7)$ & $0.028(3)$ & $0.066(4)$ & $0.061(4)$ & $0.007(3)$ & $0.000(3)$ & $-0.005(3)$ \\
\hline$C(8)$ & $0.028(3)$ & $0.057(4)$ & $0.053(3)$ & $0.004(3)$ & $-0.005(2)$ & $-0.003(2)$ \\
\hline $\mathrm{C}(9)$ & $0.039(3)$ & $0.058(4)$ & $0.060(4)$ & $0.003(3)$ & $-0.002(3)$ & $0.006(3)$ \\
\hline$C(10)$ & $0.045(3)$ & $0.074(4)$ & $0.061(4)$ & $0.003(3)$ & $-0.002(3)$ & $0.011(3)$ \\
\hline $\mathrm{C}(11)$ & $0.048(4)$ & $0.092(5)$ & $0.068(4)$ & $0.002(4)$ & $-0.014(3)$ & $0.000(3)$ \\
\hline$C(12)$ & $0.061(4)$ & $0.090(5)$ & $0.067(4)$ & $0.022(4)$ & $-0.006(3)$ & $0.014(4)$ \\
\hline$C(13)$ & $0.044(3)$ & $0.042(3)$ & $0.053(3)$ & $0.000(3)$ & $0.003(3)$ & $0.001(3)$ \\
\hline$C(14)$ & $0.055(4)$ & $0.063(4)$ & $0.079(5)$ & $0.022(4)$ & $0.007(4)$ & $0.006(3)$ \\
\hline$C(15)$ & $0.062(4)$ & $0.062(4)$ & $0.070(4)$ & $0.004(3)$ & $-0.008(3)$ & $-0.008(3)$ \\
\hline$C(16)$ & $0.051(4)$ & $0.067(5)$ & $0.096(5)$ & $-0.014(4)$ & $-0.018(4)$ & $-0.011(3)$ \\
\hline $\mathrm{C}(17)$ & $0.041(3)$ & $0.076(5)$ & $0.074(4)$ & $-0.016(4)$ & $-0.006(3)$ & $-0.001(3)$ \\
\hline$C(18)$ & $0.047(3)$ & $0.068(4)$ & $0.050(3)$ & $-0.002(3)$ & $-0.003(3)$ & $0.012(3)$ \\
\hline$C(19)$ & $0.035(3)$ & $0.062(4)$ & $0.046(3)$ & $0.002(3)$ & $-0.002(2)$ & $0.001(3)$ \\
\hline$C(20)$ & $0.034(3)$ & $0.050(3)$ & $0.051(3)$ & $-0.001(3)$ & $-0.002(2)$ & $-0.006(2)$ \\
\hline$C(21)$ & $0.095(7)$ & $0.084(6)$ & $0.150(10)$ & $0.007(6)$ & $0.040(7)$ & $-0.006(6)$ \\
\hline$C(22 A)$ & $0.030(5)$ & $0.058(6)$ & $0.056(6)$ & $-0.008(4)$ & $-0.005(4)$ & $-0.005(4)$ \\
\hline$C(22 B)$ & $0.038(12)$ & $0.18(3)$ & $0.062(14)$ & $-0.021(17)$ & $0.023(11)$ & $-0.029(16)$ \\
\hline $\mathrm{C}(23)$ & $0.077(6)$ & $0.149(10)$ & $0.104(7)$ & $0.039(7)$ & $0.005(5)$ & $0.011(6)$ \\
\hline$C(24)$ & $0.063(5)$ & $0.206(13)$ & $0.120(8)$ & $0.008(8)$ & $0.030(5)$ & $0.047(7)$ \\
\hline $\mathrm{Cl}(1)$ & $0.076(1)$ & $0.121(2)$ & $0.066(1)$ & $0.027(1)$ & $0.015(1)$ & $-0.007(1)$ \\
\hline $\mathrm{Cl}(2)$ & $0.083(1)$ & $0.101(2)$ & $0.068(1)$ & $0.013(1)$ & $-0.026(1)$ & $0.009(1)$ \\
\hline $\mathrm{N}(1)$ & $0.040(2)$ & $0.057(3)$ & $0.050(3)$ & $0.011(2)$ & $-0.002(2)$ & $-0.002(2)$ \\
\hline $\mathrm{N}(2)$ & $0.037(3)$ & $0.058(3)$ & $0.086(4)$ & $0.019(3)$ & $-0.001(3)$ & $0.006(2)$ \\
\hline $\mathrm{N}(3)$ & $0.060(3)$ & $0.058(3)$ & $0.063(3)$ & $0.017(2)$ & $-0.011(3)$ & $-0.005(3)$ \\
\hline $\mathrm{N}(4)$ & $0.108(5)$ & $0.055(4)$ & $0.120(6)$ & $0.025(4)$ & $-0.042(5)$ & $-0.035(4)$ \\
\hline $\mathrm{O}(1)$ & $0.043(2)$ & $0.046(2)$ & $0.056(2)$ & $0.005(2)$ & $0.003(2)$ & $-0.002(2)$ \\
\hline $\mathrm{O}(2)$ & $0.042(2)$ & $0.054(2)$ & $0.051(2)$ & $0.006(2)$ & $-0.006(2)$ & $-0.002(2)$ \\
\hline
\end{tabular}




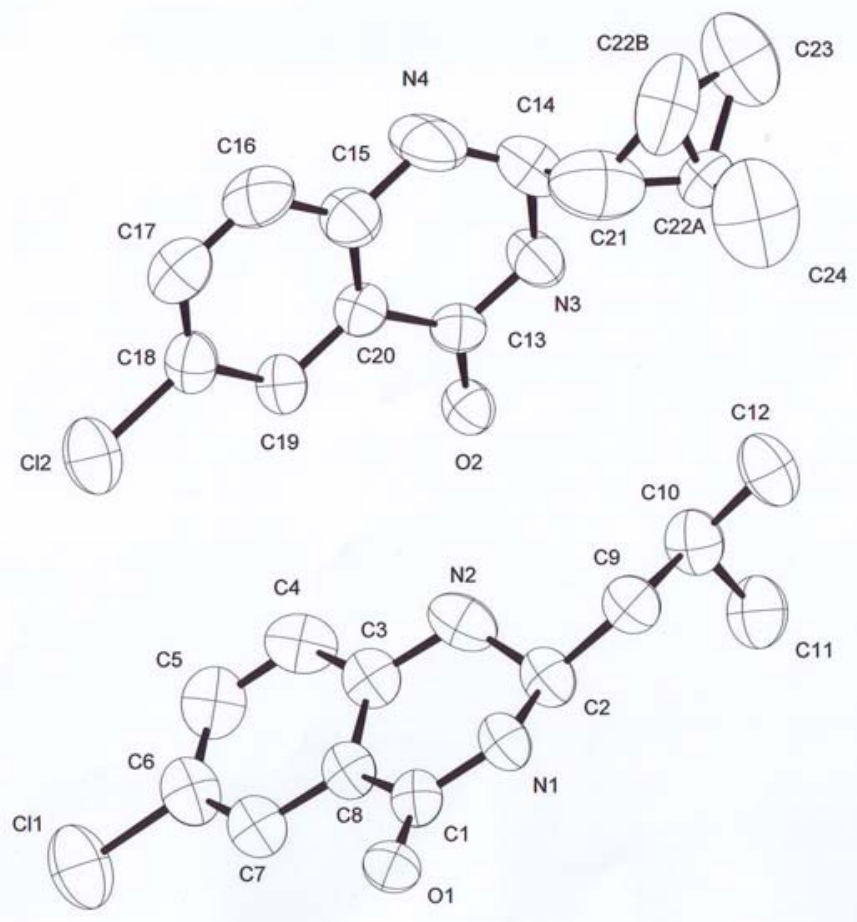

1 Jiao, P.; Nakashima, D.; Yamamoto, H. Angew. Chem. Int. Ed. 2008, 47, 2411. 